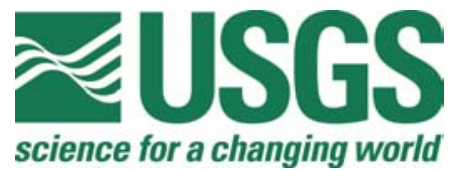

In cooperation with the U.S Department of Agriculture Forest Service

\title{
Water-Quality, Bed-Sediment, and Biological Data, for Streams in the Upper Prickly Pear Creek Watershed, Montana, 2001
}

By Terry L. Klein ${ }^{1}$, Joanna N. Thamke ${ }^{2}$, David D. Harper ${ }^{3}$, Aïda M. Farag ${ }^{3}$, David A. Nimick $^{2}$, and David L. Fey ${ }^{1}$

This report is preliminary and has not been reviewed for conformity with the U.S. Geological Survey editorial standards or with the North American Stratigraphic Code.

Any use of trade, firm, or product names is for descriptive purposes only and does not imply endorsement by the U.S. Government.

Open-File Report 2003-03-032

\footnotetext{
${ }^{1}$ Denver, Colorado

${ }^{2}$ Helena, Montana

3 Jackson, Wyoming
}

U.S. Department of the Interior

U.S. Geological Survey 


\section{CONTENTS}

Introduction.

\section{WATER-QUALITY DATA FOR STREAMS IN THE UPPER PRICKLY PEAR CREEK WATERSHED, MONTANA, 2001}

\section{By Joanna N. Thamke and David A. Nimick}

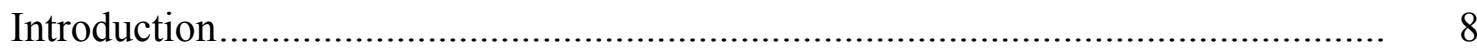

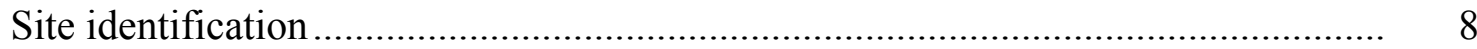

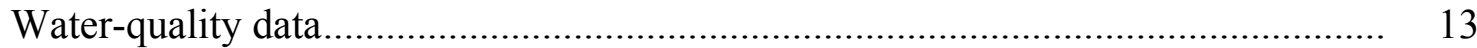

Sampling and processing methods ...................................................... 13

Routine and synoptic water-quality samples................................ 13

Diel water-quality samples...................................................... 13

Laboratory analysis ....................................................................... 14

Routine and synoptic water-quality samples................................. 14

Diel water-quality samples ...................................................... 15

Quality-assurance data ................................................................. 15

\section{BED SEDIMENT DATA FOR STREAMS IN THE UPPER PRICKLY PEAR CREEK} WATERSHED, MONTANA, 2001

By Terry L. Klein and David L. Fey

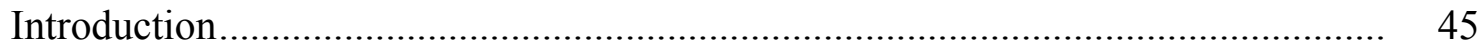

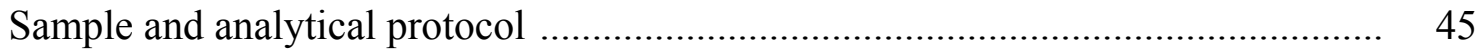

Sample locations ...................................................................... 45

Streambed sediments .......................................................................... 46

Lakebed sediments .......................................................................... 46

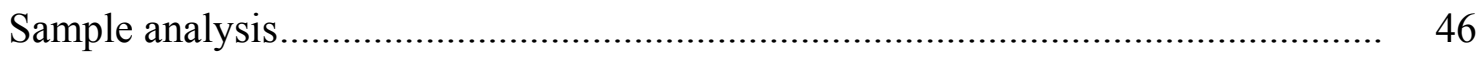

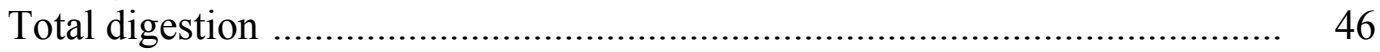

Partial digestion .................................................................................. 47

Field measurements of water samples.................................................... 47

\section{BIOLOGICAL DATA FOR STREAMS IN THE UPPER PRICKLY PEAR CREEK} WATERSHED, MONTANA, 2001

By Aïda M. Farag and David D. Harper

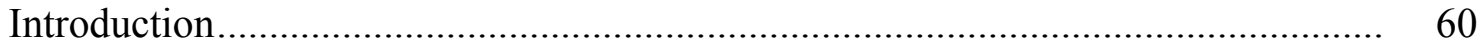

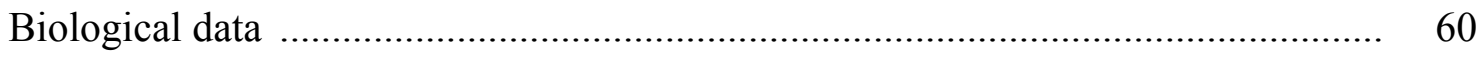

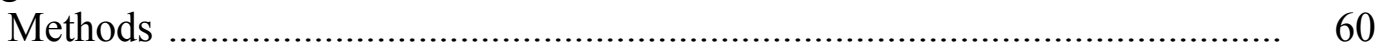

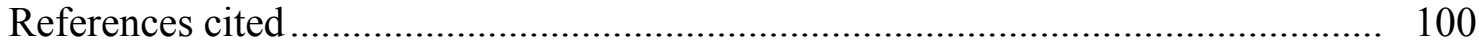




\section{ILLUSTRATIONS}

Figure 1. Map showing location of the upper Prickly Pear Creek watershed and sampling sites, Montana, 2001......................................................... 7

Figure 2. Sketch map showing approximate locations of lakebed sediment sample sites in an unnamed reservoir in upper Lump Gulch

\section{TABLES}

1. Sampling sites in the upper Prickly Pear Creek watershed, Montana, 2001

2. Water-quality data for routine and synoptic water samples collected from streams in the upper Prickly Pear Creek watershed, Montana, 2001

3. Concentrations of additional constituents at synoptic water-quality sites in the upper Prickly Pear Creek watershed, Montana, July 26-27, 2001

4. Water-quality data for diel investigations in Prickly Pear Creek at site PP-5, Montana, March-April 2001

5. Water-quality data for diel investigations in Prickly Pear Creek at site PP-4b, Montana, July 25-27, 2001

6. Water-quality data for diel investigations in Prickly Pear Creek at site PP-5, Montana, July 25-27, 2001

7. Water-quality data for diel investigations in Middle Fork Warm Springs Creek at site WS-3, Montana, June 26-27, 2001

8. Streambed sediment site locations and field measurements of water samples in the upper Prickly Pear Creek watershed, Montana, July 2001

9. Streambed sediment data using total digestion method for the upper Prickly Pear Creek watershed, Montana, July 2001

10. Lakebed sediment site locations, core descriptions and data using total digestion method for upper Prickly Pear Creek watershed, Montana, July 2001

11. Streambed sediment data using partial digestion method for the upper Prickly Pear Creek watershed, Montana, July 2001

12. Biological data for streams in the upper Prickly Pear Creek watershed, Montana, August 2001 


\section{CONVERSION FACTORS, DATUM, ABBREVIATED UNITS, AND ACRONYMS}

\begin{tabular}{rll}
\hline Multiply & \multicolumn{1}{c}{ By } & \multicolumn{1}{c}{ To obtain } \\
\hline cubic foot per second $\left(\mathrm{ft}^{3} / \mathrm{s}\right)$ & 0.028317 & cubic meter per second $\left(\mathrm{m}^{3} / \mathrm{s}\right)$ \\
foot $(\mathrm{ft})$ & 0.3048 & meter $(\mathrm{m})$ \\
inch (in.) & 25.4 & millimeter $(\mathrm{mm})$ \\
mile (mi) & 1.609 & kilometer \\
\hline
\end{tabular}

Temperature can be converted to degrees Celsius $\left({ }^{\circ} \mathrm{C}\right)$ or degrees Fahrenheit $\left({ }^{\circ} \mathrm{F}\right)$ by the following equations:

$$
\begin{aligned}
& { }^{\circ} \mathrm{C}=5 / 9\left({ }^{\circ} \mathrm{F}-32\right) \\
& { }^{\mathrm{o}} \mathrm{F}=9 / 5\left({ }^{\mathrm{o}} \mathrm{C}\right)+32
\end{aligned}
$$

Horizontal coordinate information is referenced to the North American Datum of 1927 (NAD 27).
Abbreviated units used in this report:
$\mu \mathrm{L} \quad$ microliter
$\mu \mathrm{g} / \mathrm{L} \quad$ micrograms per liter
$\mu \mathrm{m} \quad$ micrometer (micron)
$\mu \mathrm{S} / \mathrm{cm} \quad$ microsiemens per centimeter at 25 degrees Celsius
$\mathrm{meq} / \mathrm{L} \quad$ milliequivalents per liter
$\mathrm{mg} / \mathrm{L} \quad$ milligrams per liter
$\mathrm{mm} \quad$ millimeter
m meter
$\mathrm{ft} \quad$ foot
$\mathrm{nm} \quad$ nanometer
Acronyms used in this report:
$\mathrm{HCl} \quad$ Hydrochloric acid
$\mathrm{HNO}_{3} \quad$ Nitric acid
$\mathrm{HClO}_{4} \quad$ Perchloric acid
HF Hydrofluoric acid
$\mathrm{H}_{2} \mathrm{O}_{2} \quad$ Hydrogen peroxide
$\mathrm{M}$ molar
ICP-AES Inductively coupled plasma-atomic emission spectroscopy 


\section{Water-Quality, Bed-Sediment, and Biological Data, for Streams in the Upper Prickly Pear Creek Watershed, Montana, 2001}

\section{INTRODUCTION}

The upper Prickly Pear Creek watershed encompasses the upstream 15 miles of Prickly Pear Creek, south of Helena, Montana (fig. 1). The headwaters of Prickly Pear Creek and its tributaries (Beavertown Creek, Clancy Creek, Dutchman Creek, Golconda Creek, Lump Gulch, Spring Creek, and Warm Springs Creek) are primarily in the Helena National Forest, whereas the central part of the watershed primarily is within either Bureau of Land Management (BLM) or privately owned property. Three mining districts are present in the upper Prickly Pear Creek watershed: Alhambra, Clancy, and Colorado. Numerous prospects, adits, tailings piles, mills, dredge piles, and mines (mostly inactive) are located throughout the watershed. These districts contain polymetallic ( $\mathrm{Ag}, \mathrm{Au}, \mathrm{Cu}$, $\mathrm{Pb}, \mathrm{Zn})$ vein deposits and precious-metal ( $\mathrm{Au}-\mathrm{Ag}$ ) vein and disseminated deposits that were exploited beginning in the 1860's. Placer Au deposits in the major streams were extensively mined in the late 1800's and early 1900's.

As part of a cooperative effort with Federal land management agencies, the U.S. Geological Survey (USGS) is currently using an integrated approach to investigate two mining impacted watersheds in the western United States (the Animas River in Colorado and the Boulder River in Montana). These studies provide the USDA Forest Service and BLM scientific data for implementing informed land-management decisions regarding cleanup of abandoned mine lands within each watershed. A similar integrated-science approach will be used to characterize the upper Prickly Pear Creek watershed with respect to water and streambed sediment chemistry, aquatic biota, and geologic framework. This integrated database presents data that will be used to identify important pathways of metals movement and biological impacts, thereby guiding resourcemanagement decisions of land-managers in several publications that are in preparation. Watershed-level characterization in terms of water quality, streambed sediment chemistry, and fish health will facilitate determinations of whether removal of contaminated materials or other cleanup activities are necessary, planning of short- and long-term restoration efforts, and development of a monitoring plan to document cleanup effectiveness.

\section{PURPOSE AND SCOPE}

This report contains water-quality, biological, and bed-sediment data from samples collected during 2001 and is the second Open-File report for the upper Prickly Pear Creek watershed characterization. Data collected during 2000 and a summary of the geology, mineral deposits, and previous work is summarized in the first Open-File report for the upper Prickly Pear Creek watershed characterization (Klein and others, 2001). 
Data presented in this report were collected during 2001 for the following purposes: 1) to determine temporal variations in water quality, streambed sediment, and biology (metal residue in biofilm, fish tissue, and benthic macroinvertebrates, and fish health) at sites that were sampled in 2000,2) to supplement data obtained during 2000 at newly established sites, 3 ) to monitor short-term (diel) variations in water quality at selected sites, 4) to determine metal loads in upper Prickly Pear Creek at mainstem sites, and 5) to assess the fate of mining-related contaminants in a small unnamed reservoir in upper Lump Gulch. This report is organized into three sections containing 1) waterquality data, 2) streambed and lakebed sediment data, and 3) biological data. Each section contains its respective data tables.

Water-quality samples also were collected by the USGS for two site-specific studies in the watershed. A metal-loading study was conducted during June 21-27, 2001, on the Middle Fork Warm Springs Creek and a comprehensive study of the hydrogeology, water quality, and mine waste, soil, and streambed geochemistry of the Frohner Meadows area was conducted by the USGS during 2001 and 2002. The data and results from these studies are planned for separate reports 
Figure 1. Map showing location of the upper Prickly Pear Creek watershed and sampling sites, Montana, 2001

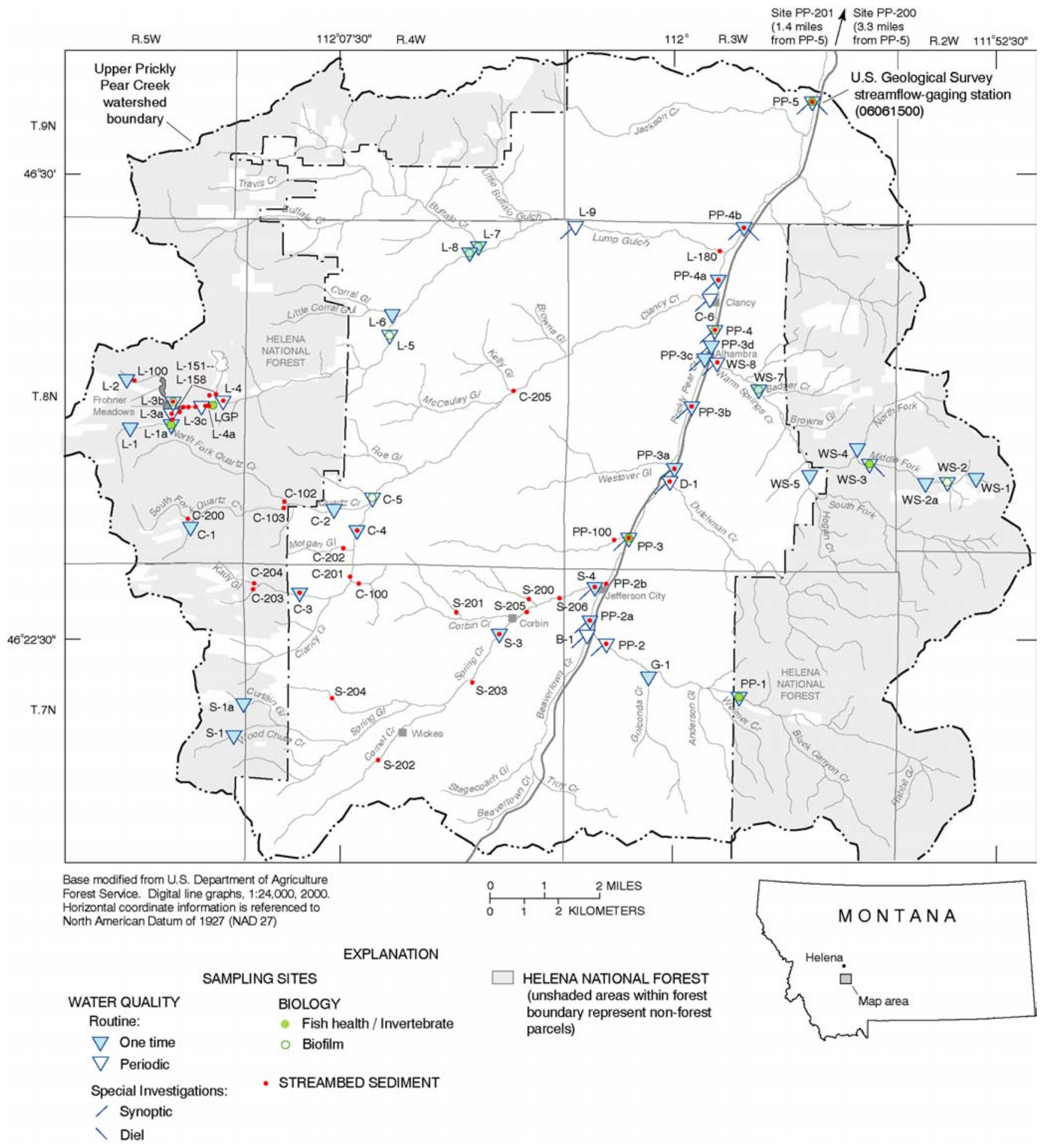




\section{WATER-QUALITY DATA FOR STREAMS IN THE UPPER PRICKLY PEAR CREEK WATERSHED, MONTANA, 2001}

By Joanna N. Thamke and David A. Nimick

\section{INTRODUCTION}

This section of the report presents water-quality and associated hydrologic data that were collected by the USGS in the upper Prickly Pear Creek watershed during the period March through August 2001. Data presented in this section of the report include measurements of streamflow, water quality, and quality assurance.

\section{SITE IDENTIFICATION}

A site number is used as the primary identification for sampling sites (fig. 1, table 1). The alpha-numeric identification allows for ease of cross reference between tables and figures. The alpha characters denote the first letters of the stream or watershed name. The numeric characters denote the downstream sequence, beginning at the most upstream site. A final alpha character ( $a, b, c$, or $d)$ in some of the site numbers denotes the sequence that the sites were added to the original site list.

Table 1. Sampling sites in the upper Prickly Pear Creek watershed, Montana, 2001

\begin{tabular}{|c|c|c|c|c|c|c|c|c|c|c|}
\hline \multirow{4}{*}{$\begin{array}{l}\text { Site } \\
\text { num- } \\
\text { ber } \\
\text { (fig. 1) }\end{array}$} & \multirow{4}{*}{ Site description } & \multirow{4}{*}{ Latitude } & \multirow{4}{*}{ Longitude } & \multicolumn{7}{|c|}{ Site Type } \\
\hline & & & & \multicolumn{4}{|c|}{ Water Quality } & \multicolumn{2}{|c|}{ Biology } & \multirow{3}{*}{$\begin{array}{l}\text { Stream- } \\
\text { bed } \\
\text { sedi- } \\
\text { ment }\end{array}$} \\
\hline & & & & \multicolumn{2}{|c|}{ Routine } & \multicolumn{2}{|c|}{$\begin{array}{c}\text { Special } \\
\text { Investigations }\end{array}$} & & & \\
\hline & & & & $\begin{array}{l}\text { One } \\
\text { time }\end{array}$ & Periodic & $\begin{array}{l}\text { Synop- } \\
\text { tic }\end{array}$ & Diel & $\begin{array}{l}\text { Fish } \\
\text { health, } \\
\text { inver- } \\
\text { tebrate }\end{array}$ & $\begin{array}{l}\text { Bio- } \\
\text { film }\end{array}$ & \\
\hline B-1 & $\begin{array}{l}\text { Beavertown Creek above Prickly Pear } \\
\text { Creek, near Jefferson City, Mont. }\end{array}$ & 462234 & 1120150 & & $\mathbf{X}$ & $\mathbf{X}$ & & & & \\
\hline $\mathrm{C}-1$ & $\begin{array}{l}\text { Unnamed tributary above South Fork } \\
\text { Quartz Creek, near Jefferson City, } \\
\text { Mont. }\end{array}$ & 462417 & 1121057 & $\mathbf{X}$ & & & & & & \\
\hline $\mathrm{C}-2$ & $\begin{array}{l}\text { Quartz Creek above Clancy Creek, } \\
\text { near Jefferson City, Mont. }\end{array}$ & 462429 & 1120804 & $\mathbf{X}$ & & & & & & \\
\hline $\mathrm{C}-3$ & $\begin{array}{l}\text { Kady Gulch above Clancy Creek, } \\
\text { near Jefferson City, Mont. }\end{array}$ & 462308 & 1120829 & $\mathbf{X}$ & & & & & & $\mathbf{X}$ \\
\hline $\mathrm{C}-4$ & $\begin{array}{l}\text { Clancy Creek above Quartz Creek, } \\
\text { near Jefferson City, Mont. }\end{array}$ & 462408 & 1120710 & $\mathbf{x}$ & & & & & & $\mathbf{x}$ \\
\hline $\mathrm{C}-5$ & $\begin{array}{l}\text { Clancy Creek below Quartz Creek, } \\
\text { near Jefferson City, Mont. }\end{array}$ & 462440 & 1120650 & & $\mathbf{X}$ & & & & $\mathbf{X}$ & \\
\hline C-6 & Clancy Creek at Clancy, Mont. & 462757 & 1115908 & & $\mathbf{X}$ & $\mathbf{X}$ & & & & \\
\hline C-100 & $\begin{array}{l}\text { Unnamed tributary below Gregory } \\
\text { Mountain, near Jefferson City, } \\
\text { Mont. }\end{array}$ & 462320 & 1120707 & & & & & & & $\mathbf{X}$ \\
\hline
\end{tabular}




\begin{tabular}{|c|c|c|c|c|c|c|c|c|c|c|}
\hline \multirow{4}{*}{$\begin{array}{c}\text { Site } \\
\text { num- } \\
\text { ber } \\
\text { (fig. 1) }\end{array}$} & \multirow{4}{*}{ Site description } & \multirow{4}{*}{ Latitude } & \multirow{4}{*}{ Longitude } & \multicolumn{7}{|c|}{ Site Type } \\
\hline & & & & \multicolumn{4}{|c|}{ Water Quality } & \multicolumn{2}{|c|}{ Biology } & \multirow{3}{*}{$\begin{array}{c}\text { Stream- } \\
\text { bed } \\
\text { sedi- } \\
\text { ment }\end{array}$} \\
\hline & & & & \multicolumn{2}{|c|}{ Routine } & \multicolumn{2}{|c|}{$\begin{array}{c}\text { Special } \\
\text { Investigations } \\
\end{array}$} & & & \\
\hline & & & & $\begin{array}{l}\text { One } \\
\text { time }\end{array}$ & Periodic & $\begin{array}{l}\text { Synop- } \\
\text { tic }\end{array}$ & Diel & $\begin{array}{l}\text { Fish } \\
\text { health, } \\
\text { inver- } \\
\text { tebrate }\end{array}$ & $\begin{array}{l}\text { Bio- } \\
\text { film }\end{array}$ & \\
\hline C-102 & $\begin{array}{l}\text { North Fork Quartz Creek above } \\
\text { South Fork Quartz Creek, near } \\
\text { Jefferson City, Mont. }\end{array}$ & 462437 & 1120851 & & & & & & & $\mathbf{X}$ \\
\hline C-103 & $\begin{array}{l}\text { South Fork Quartz Creek above } \\
\text { North Fork Quartz Creek, near } \\
\text { Jefferson City, Mont. }\end{array}$ & 462430 & 1120857 & & & & & & & $\mathbf{X}$ \\
\hline $\mathrm{C}-200$ & $\begin{array}{l}\text { South Fork Quartz Creek above } \\
\text { unnamed tributary, near Jefferson } \\
\text { City, Mont. }\end{array}$ & 462419 & 1121103 & & & & & & & $\mathbf{X}$ \\
\hline C-201 & $\begin{array}{l}\text { Clancy Creek below Gregory Mine, } \\
\text { near Jefferson City, Mont. }\end{array}$ & 462332 & 1120716 & & & & & & & $\mathbf{X}$ \\
\hline C-202 & $\begin{array}{l}\text { Morgan Gulch above Clancy Creek, } \\
\text { near Jefferson City, Mont. }\end{array}$ & 462352 & 1120717 & & & & & & & $\mathbf{x}$ \\
\hline $\mathrm{C}-203$ & $\begin{array}{l}\text { Unnamed tributary above Kady } \\
\text { Gulch, near Jefferson City, Mont. }\end{array}$ & 462316 & 1120925 & & & & & & & $\mathbf{X}$ \\
\hline C-204 & $\begin{array}{l}\text { Kady Gulch above unnamed } \\
\text { tributary, near Jefferson City, Mont. }\end{array}$ & 462318 & 1120926 & & & & & & & $\mathbf{X}$ \\
\hline C-205 & $\begin{array}{l}\text { Clancy Creek below Kelly Gulch, } \\
\text { near Clancy, Mont. }\end{array}$ & 462624 & 1120339 & & & & & & & $\mathbf{X}$ \\
\hline D-1 & $\begin{array}{l}\text { Dutchman Creek above Prickly Pear } \\
\text { Creek, near Jefferson City, Mont. }\end{array}$ & 462500 & 1115959 & & $\mathbf{X}$ & $\mathbf{X}$ & & & & $\mathbf{X}$ \\
\hline G-1 & $\begin{array}{l}\text { Golconda Creek above Prickly Pear } \\
\text { Creek, near Jefferson City, Mont. }\end{array}$ & 462156 & 1120023 & $\mathbf{X}$ & & & & & & \\
\hline LGP & $\begin{array}{l}\text { Unnamed reservoir below Park Lake, } \\
\text { near Clancy, Mont. }\end{array}$ & 462611 & 1121021 & & & & & $\mathbf{x}$ & $\mathbf{X}$ & \\
\hline L-1 & $\begin{array}{l}\text { Lump Gulch above meadows, near } \\
\text { Clancy, Mont. }\end{array}$ & 462545 & 1121222 & $\mathbf{X}$ & & & & & & \\
\hline L-1a & $\begin{array}{l}\text { Lump Gulch above Frohner Meadows } \\
\text { tributary 1, near Clancy, Mont. }\end{array}$ & 462602 & 1121121 & $\mathbf{X}$ & & & & $\mathbf{X}$ & $\mathbf{X}$ & \\
\hline L-2 & $\begin{array}{l}\text { Lump Gulch above Nellie Grant Mill, } \\
\text { near Clancy, Mont. }\end{array}$ & 462629 & 1121223 & $\mathbf{X}$ & & & & & & \\
\hline L-3a & $\begin{array}{l}\text { Lump Gulch tributary } 1 \text { below } \\
\text { Frohner Meadow, near Clancy, } \\
\text { Mont. }\end{array}$ & 462603 & 1121121 & & $\mathbf{X}$ & & & & & $\mathbf{X}$ \\
\hline L-3b & $\begin{array}{l}\text { Lump Gulch tributary } 2 \text { below } \\
\text { Frohner Meadows, near Clancy, } \\
\text { Mont. }\end{array}$ & 462612 & 1121116 & & $\mathbf{X}$ & & & & $\mathbf{X}$ & $\mathbf{X}$ \\
\hline $\mathrm{L}-3 \mathrm{c}$ & $\begin{array}{l}\text { Lump Gulch above Park Lake, near } \\
\text { Clancy, Mont. }\end{array}$ & 462607 & 1121036 & & $\mathbf{x}$ & & & & & \\
\hline L-4 & $\begin{array}{l}\text { Lump Gulch below Park Lake, near } \\
\text { Clancy, Mont. }\end{array}$ & 462612 & 1121020 & & $\mathbf{X}$ & & & & & $\mathbf{X}$ \\
\hline
\end{tabular}




\begin{tabular}{|c|c|c|c|c|c|c|c|c|c|c|}
\hline \multirow{4}{*}{$\begin{array}{c}\text { Site } \\
\text { num- } \\
\text { ber } \\
\text { (fig. 1) }\end{array}$} & \multirow{4}{*}{ Site description } & \multirow{4}{*}{ Latitude } & \multirow{4}{*}{ Longitude } & \multicolumn{7}{|c|}{ Site Type } \\
\hline & & & & \multicolumn{4}{|c|}{ Water Quality } & \multicolumn{2}{|c|}{ Biology } & \multirow{3}{*}{$\begin{array}{c}\text { Stream- } \\
\text { bed } \\
\text { sedi- } \\
\text { ment }\end{array}$} \\
\hline & & & & \multicolumn{2}{|c|}{ Routine } & \multicolumn{2}{|c|}{$\begin{array}{c}\text { Special } \\
\text { Investigations }\end{array}$} & & & \\
\hline & & & & $\begin{array}{l}\text { One } \\
\text { time }\end{array}$ & Periodic & $\begin{array}{l}\text { Synop- } \\
\text { tic }\end{array}$ & Diel & $\begin{array}{c}\text { Fish } \\
\text { health, } \\
\text { inver- } \\
\text { tebrate }\end{array}$ & $\begin{array}{l}\text { Bio- } \\
\text { film }\end{array}$ & \\
\hline $\mathrm{L}-4 \mathrm{a}$ & $\begin{array}{l}\text { Lump Gulch } 200 \text { feet above unnamed } \\
\text { reservoir, near Clancy, Mont. }\end{array}$ & 462607 & 1121018 & & & & & & & $\mathbf{X}$ \\
\hline L-5 & $\begin{array}{l}\text { Lump Gulch above Corral Gulch, } \\
\text { near Clancy, Mont. }\end{array}$ & 462727 & 1120624 & & $\mathbf{X}$ & & & & $\mathbf{X}$ & \\
\hline L-6 & $\begin{array}{l}\text { Corral Gulch above Lump Gulch, } \\
\text { near Clancy, Mont. }\end{array}$ & 462737 & 1120619 & $\mathbf{X}$ & & & & & & \\
\hline L-7 & $\begin{array}{l}\text { Lump Gulch above Buffalo Creek, } \\
\text { near Clancy, Mont. }\end{array}$ & 462839 & 1120430 & $\mathbf{X}$ & & & & & $\mathbf{X}$ & \\
\hline L-8 & $\begin{array}{l}\text { Buffalo Creek above Lump Gulch, } \\
\text { near Clancy, Mont. }\end{array}$ & 462843 & 1120427 & $\mathbf{X}$ & & & & & $\mathbf{X}$ & \\
\hline L-9 & $\begin{array}{l}\text { Lump Gulch below Little Buffalo } \\
\text { Gulch, near Clancy, Mont. }\end{array}$ & 462905 & 1120216 & & $\mathbf{X}$ & $\mathbf{X}$ & & & & \\
\hline $\mathrm{L}-100$ & $\begin{array}{l}\text { Lump Gulch east of Frohner mine, } \\
\text { near Clancy, Mont. }\end{array}$ & 462634 & 1121158 & & & & & & & $\mathbf{X}$ \\
\hline L-151 & $\begin{array}{l}\text { Lump Gulch south of Frohner } \\
\text { Meadows, near Clancy, Mont. }\end{array}$ & 462602 & 1121122 & & & & & & & $\mathbf{X}$ \\
\hline $\mathrm{L}-152$ & $\begin{array}{l}\text { Unnamed tributary west, below Park } \\
\text { Lake, near Clancy, Mont. }\end{array}$ & 462618 & 1121021 & & & & & & & $\mathbf{X}$ \\
\hline L-153 & $\begin{array}{l}\text { Unnamed tributary east, below Park } \\
\text { Lake, near Clancy, Mont. }\end{array}$ & 462612 & 1121027 & & & & & & & $\mathbf{x}$ \\
\hline L-154 & $\begin{array}{l}\text { Lump Gulch 1,100 feet above } \\
\text { unnamed reservoir, near Clancy, } \\
\text { Mont. }\end{array}$ & 462607 & 1121039 & & & & & & & $\mathbf{X}$ \\
\hline L-155 & $\begin{array}{l}\text { Lump Gulch } 1,700 \text { feet above } \\
\text { unnamed reservoir, near Clancy, } \\
\text { Mont. }\end{array}$ & 462607 & 1121050 & & & & & & & $\mathbf{X}$ \\
\hline L-156 & $\begin{array}{l}\text { Lump Gulch 2,400 feet above } \\
\text { unnamed reservoir, near Clancy, } \\
\text { Mont. }\end{array}$ & 462611 & 1121104 & & & & & & & $\mathbf{X}$ \\
\hline L-157 & $\begin{array}{l}\text { Lump Gulch } 800 \text { feet below Frohner } \\
\text { Meadows, near Clancy, Mont. }\end{array}$ & 462606 & 1121112 & & & & & & & $\mathbf{X}$ \\
\hline L-158 & $\begin{array}{l}\text { Lump Gulch } 600 \text { feet below Frohner } \\
\text { Meadows, near Clancy, Mont. }\end{array}$ & 462612 & 1121109 & & & & & & & $\mathbf{X}$ \\
\hline L-180 & $\begin{array}{l}\text { Lump Gulch above Prickly Pear } \\
\text { Creek, near Clancy, Mont. }\end{array}$ & 462843 & 1115858 & & & & & & & $\mathbf{X}$ \\
\hline PP-1 & $\begin{array}{l}\text { Prickly Pear Creek above Golconda } \\
\text { Creek, near Jefferson City, Mont. }\end{array}$ & 462133 & 1115812 & $\mathbf{X}$ & & & & $\mathbf{X}$ & $\mathbf{X}$ & \\
\hline PP-2 & $\begin{array}{l}\text { Prickly Pear Creek above Beavertown } \\
\text { Creek, near Jefferson City, Mont. }\end{array}$ & 462227 & 1120126 & & $\mathbf{X}$ & $\mathbf{X}$ & & & & $\mathbf{X}$ \\
\hline PP-2a & $\begin{array}{l}\text { Prickly Pear Creek above Spring } \\
\text { Creek, near Jefferson City, Mont. }\end{array}$ & 462241 & 1120148 & $\mathbf{X}$ & & $\mathbf{X}$ & & & & $\mathbf{X}$ \\
\hline
\end{tabular}




\begin{tabular}{|c|c|c|c|c|c|c|c|c|c|c|}
\hline \multirow{4}{*}{$\begin{array}{c}\text { Site } \\
\text { num- } \\
\text { ber } \\
\text { (fig. 1) }\end{array}$} & \multirow{4}{*}{ Site description } & \multirow{4}{*}{ Latitude } & \multirow{4}{*}{ Longitude } & \multicolumn{7}{|c|}{ Site Type } \\
\hline & & & & \multicolumn{4}{|c|}{ Water Quality } & \multicolumn{2}{|c|}{ Biology } & \multirow{3}{*}{$\begin{array}{c}\text { Stream- } \\
\text { bed } \\
\text { sedi- } \\
\text { ment }\end{array}$} \\
\hline & & & & \multicolumn{2}{|c|}{ Routine } & \multicolumn{2}{|c|}{$\begin{array}{c}\text { Special } \\
\text { Investigations }\end{array}$} & & & \\
\hline & & & & $\begin{array}{c}\text { One } \\
\text { time }\end{array}$ & Periodic & $\begin{array}{l}\text { Synop- } \\
\text { tic }\end{array}$ & Diel & $\begin{array}{c}\text { Fish } \\
\text { health, } \\
\text { inver- } \\
\text { tebrate } \\
\end{array}$ & $\begin{array}{l}\text { Bio- } \\
\text { film }\end{array}$ & \\
\hline $\mathrm{PP}-2 \mathrm{~b}$ & $\begin{array}{l}\text { Prickly Pear Creek below Spring } \\
\text { Creek }\end{array}$ & 462322 & 1120126 & & & & & & & $\mathbf{X}$ \\
\hline PP-3 & $\begin{array}{l}\text { Prickly Pear Creek near Jefferson } \\
\text { City, Mont. }\end{array}$ & 462407 & 1120052 & & $\mathbf{x}$ & $\mathbf{x}$ & & $\mathbf{x}$ & $\mathbf{X}$ & $\mathbf{x}$ \\
\hline PP-3a & $\begin{array}{l}\text { Prickly Pear Creek above Westover } \\
\text { Gulch, near Alhambra, Mont. }\end{array}$ & 462512 & 1115953 & $\mathbf{X}$ & & $\mathbf{X}$ & & & & $\mathbf{X}$ \\
\hline PP-3b & $\begin{array}{l}\text { Prickly Pear Creek above Warm } \\
\text { Springs Creek, near Alhambra, } \\
\text { Mont. }\end{array}$ & 462613 & 1115928 & $\mathbf{X}$ & & $\mathbf{X}$ & & & & $\mathbf{X}$ \\
\hline PP-3c & $\begin{array}{l}\text { Prickly Pear Creek above Warm } \\
\text { Springs Creek, at Alhambra, Mont. }\end{array}$ & 462704 & 1115910 & $\mathbf{X}$ & & $\mathbf{X}$ & & & & \\
\hline PP-3d & $\begin{array}{l}\text { Prickly Pear Creek below Warm } \\
\text { Springs Creek, at Alhambra, Mont. }\end{array}$ & 462702 & 1115907 & $\mathbf{X}$ & & $\mathbf{X}$ & & & & \\
\hline PP-4 & $\begin{array}{l}\text { Prickly Pear Creek at Alhambra RV } \\
\text { Park, near Clancy, Mont. }\end{array}$ & 462718 & 1115903 & & $\mathbf{X}$ & $\mathbf{X}$ & & & $\mathbf{X}$ & $\mathbf{X}$ \\
\hline PP-4a & $\begin{array}{l}\text { Prickly Pear Creek below Clancy } \\
\text { Creek, at Clancy, Mont. }\end{array}$ & 462807 & 1115856 & $\mathbf{X}$ & & $\mathbf{X}$ & & & & $\mathbf{X}$ \\
\hline PP-4b & $\begin{array}{l}\text { Prickly Pear Creek above Strawberry } \\
\text { Creek, near Clancy, Mont. }\end{array}$ & 462904 & 1115818 & $\mathbf{X}$ & & $\mathbf{X}$ & $\mathbf{X}$ & & & $\mathbf{X}$ \\
\hline PP-5 & $\begin{array}{l}\text { Prickly Pear Creek near Clancy, } \\
\text { Mont. }\end{array}$ & 463109 & 1115645 & & $\mathbf{X}$ & $\mathbf{X}$ & $\mathbf{X}$ & $\mathbf{X}$ & $\mathbf{X}$ & $\mathbf{X}$ \\
\hline $\begin{array}{l}\text { PP- } \\
100\end{array}$ & $\begin{array}{l}\text { Unnamed tributary to Prickly Pear } \\
\text { Creek, one mile north of Jefferson } \\
\text { City, Mont. }\end{array}$ & 462407 & 1120111 & & & & & & & $\mathbf{X}$ \\
\hline $\begin{array}{l}\text { PP- } \\
200\end{array}$ & $\begin{array}{l}\text { Prickly Pear Creek above McClellan } \\
\text { Creek, below Montana City, Mont. } \\
\text { (not shown on fig. 1) }\end{array}$ & 463315 & 1115434 & & & & & & & $\mathbf{X}$ \\
\hline $\begin{array}{l}\text { PP- } \\
201\end{array}$ & $\begin{array}{l}\text { Prickly Pear Creek above Montana } \\
\text { City, Mont. (not shown on fig. } 1 \text { ) }\end{array}$ & 463203 & 1115606 & & & & & & & $\mathbf{X}$ \\
\hline S-1 & $\begin{array}{l}\text { Wood Chute Creek above Spring } \\
\text { Creek, near Jefferson City, Mont. }\end{array}$ & 462052 & 1121000 & $\mathbf{X}$ & & & & & & \\
\hline S-1a & $\begin{array}{l}\text { Curtain Gulch above Wood Chute } \\
\text { Creek, near Jefferson City, Mont. }\end{array}$ & 462122 & 1120943 & $\mathbf{X}$ & & & & & & \\
\hline S-3 & $\begin{array}{l}\text { Spring Creek above Prickly Pear } \\
\text { Creek, near Jefferson City, Mont. }\end{array}$ & 462235 & 1120348 & $\mathbf{X}$ & & & & & & $\mathbf{X}$ \\
\hline S-4 & Spring Creek at Jefferson City, Mont. & 462320 & 1120138 & $\mathbf{X}$ & & $\mathbf{X}$ & & & & $\mathbf{X}$ \\
\hline S-200 & $\begin{array}{l}\text { Unnamed tributary above Spring } \\
\text { Creek, near Jefferson City, Mont. }\end{array}$ & 465603 & 1120304 & & & & & & & $\mathbf{X}$ \\
\hline S-201 & Corbin Creek above Corbin, Mont. & 462251 & 1120341 & & & & & & & $\mathbf{X}$ \\
\hline
\end{tabular}




\begin{tabular}{|c|c|c|c|c|c|c|c|c|c|c|}
\hline \multirow{4}{*}{$\begin{array}{c}\text { Site } \\
\text { num- } \\
\text { ber } \\
\text { (fig. 1) }\end{array}$} & \multirow{4}{*}{ Site description } & \multirow{4}{*}{ Latitude } & \multirow{4}{*}{ Longitude } & \multicolumn{7}{|c|}{ Site Type } \\
\hline & & & & \multicolumn{4}{|c|}{ Water Quality } & \multicolumn{2}{|c|}{ Biology } & \multirow{3}{*}{$\begin{array}{c}\text { Stream- } \\
\text { bed } \\
\text { sedi- } \\
\text { ment }\end{array}$} \\
\hline & & & & \multicolumn{2}{|c|}{ Routine } & \multicolumn{2}{|c|}{$\begin{array}{c}\text { Special } \\
\text { Investigations }\end{array}$} & & & \\
\hline & & & & $\begin{array}{c}\text { One } \\
\text { time }\end{array}$ & Periodic & $\begin{array}{l}\text { Synop- } \\
\text { tic }\end{array}$ & Diel & $\begin{array}{c}\text { Fish } \\
\text { health, } \\
\text { inver- } \\
\text { tebrate }\end{array}$ & $\begin{array}{l}\text { Bio- } \\
\text { film }\end{array}$ & \\
\hline S-202 & $\begin{array}{l}\text { Comet Creek above Spring Creek, } \\
\text { near Jefferson City, Mont. }\end{array}$ & 462034 & 1120633 & & & & & & & $\mathbf{X}$ \\
\hline S-203 & $\begin{array}{l}\text { Iron Ore Gulch above Spring Creek, } \\
\text { near Jefferson City, Mont. }\end{array}$ & 462142 & 1120435 & & & & & & & $\mathbf{X}$ \\
\hline S-204 & $\begin{array}{l}\text { Unnamed tributary below } \\
\text { Washington Mine, near Jefferson } \\
\text { City, Mont. }\end{array}$ & 462129 & 1120739 & & & & & & & $\mathbf{X}$ \\
\hline S-205 & $\begin{array}{l}\text { Spring Creek below Corbin Creek, } \\
\text { near Jefferson City, Mont. }\end{array}$ & 462259 & 1120311 & & & & & & & $\mathbf{X}$ \\
\hline S-206 & $\begin{array}{l}\text { Spring Creek near Corbin Flats, near } \\
\text { Jefferson City, Mont. }\end{array}$ & 462308 & 1120227 & & & & & & & $\mathbf{X}$ \\
\hline WS-1 & $\begin{array}{l}\text { Middle Fork Warm Springs Creek } \\
\text { above mines, near Clancy, Mont. }\end{array}$ & 462510 & 1115302 & $\mathbf{X}$ & & & & & & \\
\hline WS-2 & $\begin{array}{l}\text { Middle Fork Warm Springs Creek } \\
\text { below mines, near Clancy, Mont. }\end{array}$ & 462502 & 1115338 & & $\mathbf{X}$ & & & & $\mathbf{X}$ & \\
\hline WS-2a & $\begin{array}{l}\text { Middle Fork Warm Springs Creek } \\
\text { below tailings, near Clancy, Mont. }\end{array}$ & 462504 & 1115349 & $\mathbf{X}$ & & & & & & \\
\hline WS-3 & $\begin{array}{l}\text { Middle Fork Warm Springs Creek } \\
\text { near Clancy, Mont. }\end{array}$ & 462521 & 1115540 & & $\mathbf{X}$ & & $\mathbf{X}$ & $\mathbf{X}$ & $\mathbf{X}$ & \\
\hline WS-4 & $\begin{array}{l}\text { North Fork Warm Springs Creek } \\
\text { above Warm Springs Creek, near } \\
\text { Clancy, Mont. }\end{array}$ & 462529 & 1115544 & $\mathbf{x}$ & & & & & & \\
\hline WS-5 & $\begin{array}{l}\text { South Fork Warm Springs Creek near } \\
\text { Clancy, Mont. }\end{array}$ & 462511 & 1115644 & $\mathbf{X}$ & & & & & & \\
\hline WS-7 & $\begin{array}{l}\text { Warm Springs Creek above Prickly } \\
\text { Pear Creek, near Clancy, Mont. }\end{array}$ & 462633 & 1115801 & $\mathbf{x}$ & & & & & $\mathbf{X}$ & \\
\hline WS-8 & $\begin{array}{l}\text { Warm Springs Creek at Alhambra, } \\
\text { Mont. }\end{array}$ & 462700 & 1115900 & & $\mathbf{X}$ & $\mathbf{X}$ & & & & $\mathbf{X}$ \\
\hline
\end{tabular}




\section{WATER-QUALITY DATA}

Water-quality samples were collected at 45 sites located throughout the upper Prickly Pear Creek watershed during March through August 2001 (fig. 1). Samples were collected once at 28 sites and periodically at 17 sites (table 1). Seventeen of the 45 sites were sampled synoptically by the USGS on July 26 and 27 to determine principal sources of metal loads to upper Prickly Pear Creek. Water-quality data for routine (one-time or periodic) and synoptic water samples (collected within a short period of time) include field parameters (streamflow, specific conductance, $\mathrm{pH}$, and water temperature), hardness, and concentrations of selected trace elements and suspended sediment (table 2). Data for synoptic samples also include a comprehensive analysis of major-ion and traceelement concentrations (table 3 ).

Water-quality samples were collected at least hourly for diel (24-hour) investigations of water quality at three sites (PP-4b, PP-5, and WS-3) during March-July 2001 (fig. 1, tables 4-7). The sites were located along Prickly Pear and Middle Fork Warm Springs Creeks to characterize diel cycles in metal concentrations. Hourly samples were collected for about 33-47 hours at each site to describe 1-2 diel cycles. One diel sampling episode was conducted at sites PP-4b and WS-3; four diel sampling episodes were conducted at site PP-5.

\section{Sampling and Processing Methods}

\section{Routine and Synoptic Water-Quality Samples}

Composite water samples for measurements of physical properties and analyses of chemical constituents and suspended sediment were collected from multiple verticals across the stream using depth- and width-integration methods described by Wilde and others (1998). These methods provide an instantaneous mean discharge-weighted sample that is representative of the entire stream. Sampling equipment consisted of standard USGS depth-integrating suspended samplers (DH-81 and DH-48), which are constructed of plastic or coated with non-metallic epoxy paint, and equipped with a $5 / 16$-in. nylon nozzle. Grab samples were collected when streamflow was too low to allow use of the depth-integrated sampler.

Field measurements of specific conductance, $\mathrm{pH}$, and water temperature were made during sample collection. Samples were processed and prepared for laboratory analysis according to procedures described by Wilde and others (1998). Routine and synoptic samples for dissolved constituents were filtered using a $0.45-\mu \mathrm{m}$ pore-size capsule filter. Instantaneous streamflow at the time of sample collection was determined by direct measurement, estimation, or stage-discharge rating (Rantz and others, 1982).

\section{Diel Water-Quality Samples}

An automatic pumping sampler was used to collect diel samples. Samples typically were collected hourly during each diel sampling episode, although some samples were collected every 30 minutes. All hoses and polyethylene collection bottles used with the automatic sampler were cleaned and acid rinsed between sampling episodes. Hoses and bottles were rinsed with stream water by the automatic pumping sampler pre-rinse program before sample collection. The intake for the automatic sampler was positioned 2 to $6 \mathrm{in}$. above the streambed in a riffle where the stream 
velocity was sufficient to ensure downstream flushing of rinse water expelled by the sampler prior to collection of the sample. Collection bottles were removed from the automatic sampler within several hours of collection and taken to a field laboratory, where they were processed (split into aliquots, measured for onsite parameters, filtered and preserved) according to procedures described by Wilde and others (1998). During diel sampling on July 25-27, 2001, at sites PP-4b and PP-5, collection bottles were removed and processed within one hour to minimize changes in concentrations of arsenic and iron species. Diel samples for dissolved constituents were filtered through $0.1-\mu \mathrm{m}$ pore-size capsule or plate filters. Aliquots for analysis of arsenic and iron species were stored chilled in opaque sample bottles after preservation with hydrochloric acid.

Aliquots for analysis of cations and other trace elements were preserved with nitric acid. Aliquots for analysis of major anions were not preserved.

Water temperature, specific conductance, and dissolved oxygen were measured hourly (or more frequently) with a multi-parameter instrument submerged in the stream in an area of slow-moving water near the intake of the pumping sampler. Stream $\mathrm{pH}$ was measured either with the instream multi-parameter instrument or with a laboratory $\mathrm{pH}$ meter using water samples that were manually collected from the stream and immediately analyzed. In some instances, both types of $\mathrm{pH}$ measurements were made to provide comparisons of results. Streamflow for site PP-5 was determined from data for USGS streamflow-gaging station 06061500. Streamflow for site PP-4b was estimated from hourly stage data and a stage-discharge relation developed from periodic current-meter measurements (Rantz and others 1982). Streamflow for site WS-3 was determined by continuous tracer injection (T.E. Cleasby, U.S. Geological Survey, unpub. data, 2001).

\section{Laboratory Analysis}

\section{Routine and Synoptic Water-Quality Samples}

Trace elements selected for analysis of routine samples were similar to those analyzed for another abandoned mine lands study in the Boulder River watershed, Montana, and include total-recoverable and dissolved concentrations of arsenic, cadmium, copper, lead, and zinc. Trace elements that consistently had concentrations less than the minimum reporting level in the Boulder River watershed (Nimick and Cleasby, 2000) were not analyzed. Analysis of an extensive suite of trace elements in water samples collected from five sites in the upper Prickly Pear Creek watershed in October 2000 confirmed non-detectable concentrations for the omitted elements (Klein and others, 2001, table 3).

All routine water-quality samples and selected replicate synoptic samples were analyzed by the USGS National Water Quality Laboratory (NWQL) in Denver, Colo., according to methods described by Fishman and Freidman (1989), Faires (1993), Fishman (1993), Hoffman and others (1996), Garbarino and Struzeski (1998), Garbarino (1999), and Jones and Garbarino (1999). Routine samples were analyzed for hardness and concentrations of selected trace elements (table 2). Selected replicate synoptic samples were analyzed for total-recoverable concentrations of selected trace elements.

Seventeen of the 45 sites were sampled synoptically (all samples collected in a short period of time) on July 26-27, 2001 (tables 2 and 3). These samples were analyzed by the USGS laboratory in Boulder, Colo. Concentrations of major cations and trace elements were determined using inductively coupled plasma-optical emission 
spectrometry. Major cations were analyzed using the radial view while the axial view was used for trace elements. Total recoverable concentrations of arsenic were measured by a flow-injection analysis system for the generation of arsine and detection using atomic absorption spectrometry (McCleskey and others, 2001). Cadmium, copper, and lead concentrations were measured by graphite furnace atomic absorption spectrometry using a $\mathrm{Pd} / \mathrm{Mg}\left(\mathrm{NO}_{3}\right)_{2}$ matrix modifier, an atomization temperature of $1,900^{\circ} \mathrm{C}$, transverse heated graphite atomizer, and Zeeman-effect and continuum-source background corrections. Concentrations of major anions were determined using an ion chromatograph (Brinton and others, 1995). Samples were analyzed using 10- $\mu \mathrm{L}$ and 50$\mu \mathrm{L}$ sample loops. For samples that contained measured concentrations of anions and cations, the data were checked using the program WATEQ4F (Ball and Nordstrom, 1991) for adequate charge balance.

Water samples for suspended-sediment concentration and the percentage of suspended sediment finer than 0.062-mm diameter (silt size and smaller) were analyzed by the USGS Montana District sediment laboratory in Helena, Mont., according to gravimetric methods described by Guy (1969) and Lambing and Dodge (1993).

\section{Diel Water-Quality Samples}

Diel water-quality samples collected in March and April 2001 at site PP-5 were analyzed for dissolved $\mathrm{Zn}$ by inductively coupled plasma-mass spectrometry (Garbarino and Taylor, 1996) by the NWQL (table 4). Diel water-quality samples collected in July 2001 at site PP-4b and PP-5 (tables 5 and 6) were analyzed by the USGS laboratory in Boulder, Colo. In addition to the analytical methods described for the synoptic waterquality samples, iron concentrations were determined using a modification of the FerroZine colorimetric method (Stookey, 1970). Alkalinity was determined by autotitration using standardized $\mathrm{H}_{2} \mathrm{SO}_{4}$ (Barringer and Johnsson, 1996). Diel water-quality samples collected in June 2001 at site WS-3 were analyzed by the USGS laboratory in Boulder, Colo. (table 7).

\section{Quality-Assurance Data}

Data-collection and analytical procedures used during this study incorporated practices designed to control, verify, and assess the quality of sample data. Qualityassurance procedures used for the collection and field processing of water-quality samples are described by Knapton and Nimick (1991) and Wilde and others (1998). Standard procedures used by the NWQL for internal sample handling and quality assurance are described by Friedman and Erdmann (1982), Jones (1987), and Pritt and Raese (1995). The Montana District sediment laboratory uses quality-assurance procedures described by Lambing and Dodge (1993).

The USGS laboratory in Boulder, Colo. ensured that all reagents were of purity at least equal to the reagent-grade standards of the American Chemical Society. Double distilled water and re-distilled acids were used in all preparations. USGS standard reference water samples were used as independent standards to ensure accuracy. A stream sample was analyzed numerous times to monitor precision during and between analytical runs. Each sample was analyzed in at least duplicate for major cations and trace metals. Samples were diluted as necessary to bring the analyte concentration within the optimal range of the method. 
Replicate samples for this study are two or more samples considered to be essentially identical in composition. Replicate samples were obtained in the field by splitting a single composite sample into two or more subsamples, which then were analyzed separately. Analyses of field replicates are noted in tables 2, 3, 5, and 6.

A blank sample of deionized water was routinely analyzed to identify the presence and magnitude of contamination that potentially could bias analytical results. The particular type of blank sample routinely tested was a "field" blank. A field blank is an aliquot of deionized water, which is certified as trace-element free and is processed through the sampling equipment used to collect stream samples. The blank is then subjected to the same processing (filtration, preservation, transportation, and laboratory handling) as stream samples. The blank sample was analyzed for the same constituents as those of stream samples to identify whether any detectable concentrations exist. Analytical results for field blanks are noted in tables 2, 3, 5, and 6.

The NWQL collects quality-control data on a continuing basis to evaluate selected analytical methods to determine laboratory reporting levels (LRL). Accordingly, concentrations are reported as $<$ LRL for samples in which the analyte was either not detected or could not be reliably identified. Analytes that are detected at concentrations less than LRL and that pass identification criteria are reported with an estimated concentration. Estimated concentrations are noted in data tables with a remark code of "E." Those data should be used with the understanding that their uncertainty of quantification is greater than that of data reported without the "E" remark code. 
Table 2. Water-quality data for routine and synoptic water samples collected from streams in the upper Prickly Pear Creek watershed, Montana, 2001.

[Samples analyzed by the U.S. Geological Survey. Analytical data are reported to a maximum of three significant figures to provide sufficient resolution for detecting small differences between samples closely spaced in time or distance. Abbreviations: B, blank sample; Boulder, USGS laboratory in Boulder, Colo.; E, estimated; $\mathrm{ft}^{3} / \mathrm{s}$, cubic feet per second; ${ }^{\circ} \mathrm{C}$, degrees Celsius; g/L, micrograms per liter; S/cm, microsiemens per centimeter at $25^{\circ} \mathrm{C} ; \mathrm{mg} / \mathrm{L}$, milligrams per liter; mm, millimeters; NWQL, USGS National Water Quality Laboratory in Denver, Colo.; QA, quality assurance; R, replicate sample; su, standard units. Symbols: $<$, less than minimum reporting level; --, no data].

\begin{tabular}{|c|c|c|c|c|c|c|c|c|c|c|c|c|c|}
\hline $\begin{array}{c}\text { Site } \\
\text { number }\end{array}$ & $\begin{array}{c}\text { QA } \\
\text { sample } \\
\text { type }\end{array}$ & $\begin{array}{l}\text { Labora- } \\
\text { tory }\end{array}$ & Time & $\begin{array}{c}\text { Stream- } \\
\text { flow } \\
\left(\mathbf{f t}^{3} / \mathbf{s}\right)\end{array}$ & $\begin{array}{l}\text { Specific con- } \\
\text { ductance, } \\
\text { field }(\mu S / \mathrm{cm})\end{array}$ & $\begin{array}{l}\text { pH, } \\
\text { field } \\
\text { (su) }\end{array}$ & $\begin{array}{c}\text { Temp- } \\
\text { erature, } \\
\text { water } \\
\left({ }^{\circ} \mathrm{C}\right)\end{array}$ & $\begin{array}{c}\text { Hard- } \\
\text { ness } \\
(\mathrm{mg} / \mathrm{L})\end{array}$ & $\begin{array}{c}\text { Calcium, } \\
\text { dissolved } \\
(\mathrm{mg} / \mathrm{L})\end{array}$ & $\begin{array}{l}\text { Magne- } \\
\text { sium, } \\
\text { dissolved } \\
(\mathrm{mg} / \mathrm{L})\end{array}$ & $\begin{array}{c}\text { Sediment, } \\
\text { suspended } \\
(\mathrm{mg} / \mathrm{L})\end{array}$ & $\begin{array}{l}\text { Sediment, } \\
\text { suspended, } \\
\text { diameter } \\
\text { (percent } \\
\text { finer than } \\
\text { 0.062 mm) }\end{array}$ & $\begin{array}{l}\text { Arsenic, total } \\
\text { recov-erable } \\
(\mu \mathrm{g} / \mathrm{L})\end{array}$ \\
\hline B-1 & & NWQL & $2001051 C 1240$ & 0.95 & 338 & 7.9 & 10.0 & 137 & 39.7 & 9.16 & 4 & 71 & $<2$ \\
\hline B-1 & & Boulder & 200107260945 & 0.22 & 372 & 7.7 & 11.5 & 183 & 53.2 & 12.2 & -- & -- & 0.600 \\
\hline $\mathrm{C}-1$ & & NWQL & $2001051 C 1430$ & 0.65 & 88 & 7.3 & 2.8 & 35 & 11.1 & 1.78 & 26 & 36 & E1.80 \\
\hline $\mathrm{C}-2$ & & NWQL & $2001050 \subseteq 1315$ & 6.04 & 102 & -- & 5.0 & 43 & 13.6 & 2.32 & 16 & 43 & E1.44 \\
\hline $\mathrm{C}-3$ & & NWQL & $2001050 \subseteq 1025$ & 0.78 & 134 & 8.5 & 3.5 & 51 & 16.3 & 2.46 & 5 & 59 & $<2$ \\
\hline C-4 & & NWQL & $2001050 \subseteq 1130$ & 0.28 & 258 & 8.0 & 8.5 & 105 & 32.2 & 6.08 & 2 & 73 & 22.6 \\
\hline C-5 & & NWQL & $2001040 \leqq 1330$ & 0.78 & 215 & 8.2 & 4.0 & 97 & 29.0 & 5.96 & 3 & 52 & 4.99 \\
\hline C-5 & & NWQL & $2001050 \subseteq 1400$ & 6.63 & 120 & 7.8 & 7.0 & 50 & 15.4 & 2.83 & 10 & 77 & 3.65 \\
\hline C-5 & B & NWQL & $2001051 C 0700$ & -- & 2 & 5.6 & -- & -- & E0.01 & $<0.008$ & -- & -- & $<6$ \\
\hline C-6 & & NWQL & $2001051 C 1700$ & 6.9 & 180 & 8.0 & 10.0 & 75 & 22.1 & 4.89 & 26 & 40 & 6.49 \\
\hline C-6 & & Boulder & 200107261430 & 0.83 & 243 & 7.8 & 16.5 & 110 & 31.8 & 7.57 & -- & -- & 7.03 \\
\hline C-6 & $\mathrm{R}$ & NWQL & 200107261435 & 0.83 & 243 & 7.8 & 16.5 & -- & -- & -- & -- & -- & 7.50 \\
\hline D-1 & & NWQL & $2001051 C 0800$ & 5.44 & 71 & 7.8 & 2.5 & 28 & 8.28 & 1.67 & 10 & 60 & E0.905 \\
\hline D-1 & & Boulder & 200107261120 & 1.2 & 90 & 7.5 & 16.0 & 38 & 11.5 & 2.31 & -- & -- & 0.535 \\
\hline G-1 & & NWQL & $2001051 C 1145$ & 1.63 & 83 & 7.8 & 4.0 & 32 & 10.4 & 1.52 & 3 & 64 & $<2$ \\
\hline L-1 & & NWQL & $2001051 C 1000$ & 1.5 & 39 & 8.7 & 0.0 & 15 & 4.98 & 0.68 & 23 & 59 & 3.21 \\
\hline L-1a & & NWQL & 200108011215 & 0.37 & 68 & 7.6 & 9.0 & 28 & 8.95 & 1.31 & 2 & 40 & 1.94 \\
\hline $\mathrm{L}-2$ & & NWQL & $2001050 \subseteq 1100$ & 0.18 & 51 & 7.8 & 7.6 & 20 & 6.69 & 0.85 & 5 & 69 & 2.06 \\
\hline L-3a & & NWQL & $2001051 C 1100$ & 0.26 & 88 & 6.3 & 4.5 & 29 & 9.35 & 1.38 & 2 & 94 & 7.37 \\
\hline L-3a & & NWQL & 200108011130 & 0.004 & 94 & 7.7 & 8.5 & 33 & 10.8 & 1.52 & 2 & 79 & 11.2 \\
\hline L-3b & & NWQL & 200103151130 & 0.060 & 193 & 7.5 & 0.1 & 72 & 23.6 & 3.19 & -- & -- & 16.7 \\
\hline L-3b & & NWQL & $2001051 C 1120$ & 0.78 & 100 & 6.3 & 5.0 & 32 & 10.4 & 1.42 & 3 & 88 & 14.4 \\
\hline L-3b & $\mathrm{R}$ & NWQL & $2001051 C 1121$ & 0.78 & 100 & 6.3 & 5.0 & 32 & 10.3 & 1.41 & -- & -- & 14.8 \\
\hline$L-3 b$ & & NWQL & 200108010950 & 0.23 & 121 & 8.2 & 10.0 & 42 & 13.6 & 1.91 & 3 & 55 & 33.5 \\
\hline L-3c & & NWQL & $2001050 \subseteq 1245$ & 4.1 & 64 & 6.8 & 7.0 & 23 & 7.30 & 1.11 & 11 & 71 & 4.45 \\
\hline L-3c & & NWQL & 200108011515 & 0.38 & 92 & 7.7 & 15.5 & 35 & 11.2 & 1.79 & 3 & 85 & 5.23 \\
\hline L-4 & & NWQL & $2001050 \subseteq 130$ & 1.3 & 70 & 6.7 & 4.0 & 25 & 8.05 & 1.29 & 13 & 63 & 5.53 \\
\hline L-4 & & NWQL & 2001080114003 & 1.52 & 88 & 8.0 & 13.5 & 33 & 10.1 & 1.87 & 4 & 60 & 3.95 \\
\hline L-5 & & NWQL & $2001040 \leq 1530$ & 2.02 & 149 & 7.9 & -- & 61 & 18.2 & 3.78 & 7 & 59 & E1.47 \\
\hline L-5 & & NWQL & $2001051 C 0915$ & 7.81 & 94 & 8.2 & 4.5 & 35 & 10.8 & 2.07 & 4 & 81 & E1.88 \\
\hline
\end{tabular}


Table 2. Water-quality data for routine and synoptic water samples collected from streams in the upper Prickly Pear Creek watershed, Montana, 2001 (Continued).

\begin{tabular}{|c|c|c|c|c|c|c|c|c|c|c|c|c|c|c|}
\hline $\begin{array}{c}\text { Site } \\
\text { number }\end{array}$ & $\begin{array}{c}\text { QA } \\
\text { sample } \\
\text { type }\end{array}$ & $\begin{array}{c}\text { Labora- } \\
\text { tory }\end{array}$ & Date & Time & $\begin{array}{l}\text { Arsenic, } \\
\text { dis } \\
\text { solved } \\
(\mu \mathrm{g} / \mathrm{L})\end{array}$ & $\begin{array}{c}\text { Cad- } \\
\text { mium, } \\
\text { total } \\
\text { recov- } \\
\text { erable } \\
(\mu \mathrm{g} / \mathrm{L})\end{array}$ & $\begin{array}{c}\text { Cad- } \\
\text { mium, } \\
\text { dis- } \\
\text { solved } \\
(\mu \mathrm{g} / \mathrm{L})\end{array}$ & $\begin{array}{l}\text { Cop- } \\
\text { per, } \\
\text { total } \\
\text { recov- } \\
\text { erable } \\
(\mu \mathrm{g} / \mathrm{L})\end{array}$ & $\begin{array}{c}\text { Cop- } \\
\text { per, dis- } \\
\text { solved } \\
(\mu \mathrm{g} / \mathrm{L})\end{array}$ & $\begin{array}{l}\text { Lead, } \\
\text { total } \\
\text { recov- } \\
\text { erable } \\
(\mu \mathrm{g} / \mathrm{L})\end{array}$ & $\begin{array}{c}\text { Lead, } \\
\text { dis- } \\
\text { solved } \\
(\mu \mathrm{g} / \mathrm{L})\end{array}$ & $\begin{array}{c}\text { Molyb- } \\
\text { denum, } \\
\text { total } \\
\text { recov- } \\
\text { erable } \\
(\mu \mathrm{g} / \mathrm{L})\end{array}$ & $\begin{array}{l}\text { Zinc, } \\
\text { total } \\
\text { recov- } \\
\text { erable } \\
(\mu \mathrm{g} / \mathrm{L})\end{array}$ & $\begin{array}{c}\text { Zinc, dis- } \\
\text { solved } \\
(\mu \mathrm{g} / \mathrm{L})\end{array}$ \\
\hline B-1 & & NWQL & 20010510 & 1240 & 0.342 & 0.119 & 0.077 & 4.36 & 3.50 & $<1$ & $<0.08$ & 7.79 & 42.8 & 36.4 \\
\hline B-1 & & Boulder & 20010726 & 0945 & 0.486 & 0.138 & 0.087 & 5.69 & 3.50 & 0.170 & $<0.1$ & -- & 42.9 & 22.6 \\
\hline $\mathrm{C}-1$ & & NWQL & 20010510 & 1430 & 0.943 & 0.370 & 0.173 & 2.87 & 2.17 & 5.24 & 0.301 & -- & 71.0 & 50.8 \\
\hline $\mathrm{C}-2$ & & NWQL & 20010509 & 1315 & 0.725 & 0.140 & E0.035 & 2.31 & 1.59 & 2.19 & 0.143 & -- & 24.4 & 9.38 \\
\hline $\mathrm{C}-3$ & & NWQL & 20010509 & 1025 & 0.589 & 0.076 & E0.028 & 0.951 & 0.838 & 1.17 & $<0.08$ & -- & 19.9 & 13.5 \\
\hline $\mathrm{C}-4$ & & NWQL & 20010509 & 1130 & 20.2 & 1.36 & 1.35 & 1.97 & 4.31 & $<1$ & 0.087 & -- & 319 & 324 \\
\hline$C-5$ & & NWQL & 20010405 & 1330 & 3.67 & 0.126 & 0.078 & 1.97 & 1.24 & 1.50 & 0.083 & -- & 24.1 & 20.1 \\
\hline C-5 & & NWQL & 20010509 & 1400 & 2.04 & 0.190 & 0.060 & 2.69 & 1.82 & 2.70 & 0.171 & -- & 29.4 & 14.7 \\
\hline$C-5$ & B & NWQL & 20010510 & 0700 & $<0.4$ & $<0.04$ & $<0.07$ & $<0.6$ & E0.3 & $<1$ & $<0.2$ & -- & $<1$ & 2 \\
\hline C-6 & & NWQL & 20010510 & 1700 & 4.74 & 0.223 & 0.083 & 3.80 & 2.19 & 4.28 & 0.138 & -- & 40.6 & 16.8 \\
\hline C-6 & & Boulder & 20010726 & 1430 & 5.93 & 0.089 & $<0.05$ & 1.95 & 1.99 & 0.529 & $<0.1$ & -- & 10.3 & 8.50 \\
\hline C-6 & $\mathrm{R}$ & NWQL & 20010726 & 1435 & -- & 0.072 & -- & 3.56 & -- & $<1$ & -- & -- & 12.4 & -- \\
\hline D-1 & & NWQL & 20010510 & 0800 & 0.993 & $<0.04$ & $<0.04$ & 1.85 & 1.47 & $<1$ & $<0.08$ & -- & $<1$ & $<1$ \\
\hline D-1 & & Boulder & 20010726 & 1120 & 0.447 & $<0.05$ & $<0.05$ & 1.73 & 1.69 & $<0.1$ & $<0.1$ & -- & $<1$ & 1.10 \\
\hline G-1 & & NWQL & 20010510 & 1145 & 0.749 & 0.168 & 0.146 & 1.52 & 1.34 & 3.17 & 1.04 & -- & 27.1 & 26.2 \\
\hline L-1 & & NWQL & 20010510 & 1000 & 1.76 & 0.114 & 0.073 & 3.96 & 3.06 & 2.14 & 0.227 & -- & 12.7 & 11.8 \\
\hline L-1a & & NWQL & 20010801 & 1215 & 1.06 & E0.029 & $<0.04$ & 1.54 & 1.29 & $<1$ & $<0.08$ & -- & 4.95 & 2.26 \\
\hline L-2 & & NWQL & 20010509 & 1100 & 1.39 & E0.023 & $<0.04$ & 3.15 & 2.10 & $<1$ & 0.108 & -- & 2.77 & 1.23 \\
\hline L-3a & & NWQL & 20010510 & 1100 & 4.44 & 6.66 & 6.33 & 7.29 & 6.86 & 2.39 & 0.848 & -- & 1,630 & 1,660 \\
\hline $\mathrm{L}-3 \mathrm{a}$ & & NWQL & 20010801 & 1130 & 3.24 & 5.21 & 4.58 & 6.87 & 5.09 & 1.57 & E0.067 & -- & 1,360 & 1,190 \\
\hline L-3b & & NWQL & 20010315 & 1130 & 4.84 & 15.6 & 15.5 & 10.3 & 5.28 & 9.34 & 2.36 & -- & 4,720 & 4,810 \\
\hline $\mathrm{L}-3 \mathrm{~b}$ & & NWQL & 20010510 & 1120 & 8.01 & 13.1 & 13.3 & 11.4 & 10.5 & 7.14 & 2.41 & 0.255 & 2,510 & 2,590 \\
\hline $\mathrm{L}-3 \mathrm{~b}$ & $\mathrm{R}$ & NWQL & 20010510 & 1121 & 7.57 & 13.1 & 12.8 & 12.6 & 10.3 & 7.66 & 2.57 & -- & 2,540 & 2,580 \\
\hline $\mathrm{L}-3 \mathrm{~b}$ & & NWQL & 20010801 & 0950 & 10.5 & 4.99 & 4.13 & 6.48 & 3.25 & 7.83 & 1.53 & -- & 1,190 & 1,070 \\
\hline $\mathrm{L}-3 \mathrm{c}$ & & NWQL & 20010509 & 1245 & 2.27 & 2.61 & 2.49 & 5.67 & 4.28 & 2.29 & 0.440 & -- & 706 & 688 \\
\hline $\mathrm{L}-3 \mathrm{c}$ & & NWQL & 20010801 & 1515 & 1.90 & 1.24 & 0.950 & 2.26 & 1.56 & $<1$ & E0.063 & -- & 318 & 279 \\
\hline L-4 & & NWQL & 20010509 & 1330 & 1.97 & 2.58 & 1.74 & 5.17 & 3.54 & 2.79 & 0.345 & -- & 631 & 532 \\
\hline L-4 & & NWQL & 20010801 & 1400 & 2.33 & 0.794 & 0.156 & 1.89 & 1.30 & $<1$ & 0.106 & -- & 144 & 63.0 \\
\hline L-5 & & NWQL & 20010405 & 1530 & 0.521 & 0.183 & 0.068 & 1.34 & 0.765 & $<1$ & $<0.08$ & -- & 89.6 & 59.9 \\
\hline L-5 & & NWQL & 20010510 & 0915 & 1.11 & 0.307 & 0.228 & 2.66 & 2.22 & $<1$ & 0.149 & -- & 222 & 209 \\
\hline
\end{tabular}


Table 2. Water-quality data for routine and synoptic water samples collected from streams in the upper Prickly Pear Creek watershed, Montana, 2001 (Continued).

\begin{tabular}{|c|c|c|c|c|c|c|c|c|c|c|c|c|c|c|}
\hline $\begin{array}{c}\text { Site } \\
\text { number }\end{array}$ & $\begin{array}{c}\text { QA } \\
\text { sample } \\
\text { type }\end{array}$ & $\begin{array}{c}\text { Labora- } \\
\text { tory }\end{array}$ & Date & Time & $\begin{array}{c}\text { Stream- } \\
\text { flow } \\
\left(\mathrm{ft}^{3} / \mathbf{s}\right)\end{array}$ & $\begin{array}{c}\text { Specific } \\
\text { con- } \\
\text { ductance, } \\
\text { field }(\mu \mathrm{S} / \mathrm{cm})\end{array}$ & $\begin{array}{l}\mathrm{pH}, \\
\text { field } \\
\text { (su) }\end{array}$ & $\begin{array}{l}\text { Temp- } \\
\text { erature, } \\
\text { water } \\
\left({ }^{\circ} \mathrm{C}\right)\end{array}$ & $\begin{array}{c}\text { Hard- } \\
\text { ness } \\
(\mathrm{mg} / \mathrm{L})\end{array}$ & $\begin{array}{c}\text { Calcium, } \\
\text { dissolved } \\
(\mathrm{mg} / \mathrm{L})\end{array}$ & $\begin{array}{l}\text { Magne- } \\
\text { sium, } \\
\text { dissolved } \\
(\mathrm{mg} / \mathrm{L})\end{array}$ & $\begin{array}{c}\text { Sediment, } \\
\text { suspended } \\
(\mathrm{mg} / \mathrm{L})\end{array}$ & $\begin{array}{l}\text { Sediment, } \\
\text { suspended, } \\
\text { diameter } \\
\text { (percent } \\
\text { finer than } \\
\text { 0.062 mm) }\end{array}$ & $\begin{array}{l}\text { Arsenic, } \\
\text { total recov- } \\
\text { erable } \\
(\mu \mathrm{g} / \mathrm{L})\end{array}$ \\
\hline L-6 & & NWQL & 20010510 & 1000 & 1.07 & 146 & 7.7 & 5.0 & 47 & 13.8 & 3.10 & 3 & 66 & $<2$ \\
\hline L-7 & & NWQL & 20010510 & 1100 & 7.93 & 106 & 7.4 & 6.0 & 39 & 11.9 & 2.35 & 3 & 74 & E1.37 \\
\hline L-7 & $\mathrm{R}$ & NWQL & 20010510 & 1105 & 7.93 & -- & -- & -- & 39 & 11.8 & 2.36 & -- & -- & E1.21 \\
\hline L-8 & & NWQL & 20010510 & 1045 & 3.85 & 201 & 7.7 & 6.5 & 68 & 20.0 & 4.47 & 6 & 81 & $<2$ \\
\hline L-9 & & NWQL & 20010510 & 1215 & 11.9 & 147 & 7.7 & 9.0 & 53 & 15.7 & 3.32 & 5 & 69 & E0.925 \\
\hline L-9 & & Boulder & 20010726 & 1520 & 1.37 & 139 & 7.6 & 20.0 & 60 & 17.7 & 3.87 & -- & -- & 0.681 \\
\hline L-9 & B & Boulder & 20010726 & 1525 & -- & -- & -- & -- & -- & $<0.05$ & $<0.06$ & -- & -- & $<0.1$ \\
\hline PP-1 & & NWQL & 20010510 & 0915 & 9.44 & 69 & 8.1 & 2.5 & 29 & 9.07 & 1.45 & 2 & 68 & $<2$ \\
\hline PP-2 & & NWQL & 20010510 & 1040 & 13.2 & 82 & 7.9 & 5.0 & 33 & 10.4 & 1.67 & 4 & 50 & $<2$ \\
\hline PP-2 & & NWQL & 20010514 & 1130 & 22.2 & 61 & 7.9 & 7.5 & 25 & 8.00 & 1.26 & 15 & 60 & E1.63 \\
\hline PP-2 & & Boulder & 20010726 & 0815 & 8.06 & 82.9 & 7.4 & 9.5 & 38 & 12.1 & 1.95 & -- & -- & 0.427 \\
\hline PP-2a & & Boulder & 20010726 & 0740 & 8.54 & 98.8 & 7.4 & 9.5 & 45 & 14.1 & 2.44 & -- & -- & 0.517 \\
\hline PP-2a & $\mathrm{R}$ & Boulder & 20010726 & 0741 & 8.54 & 98.8 & 7.4 & 9.5 & 46 & 14.2 & 2.45 & -- & -- & 0.519 \\
\hline PP-2a & $\mathrm{R}$ & NWQL & 20010726 & 0741 & 8.54 & 98.8 & 7.4 & 9.5 & -- & -- & -- & -- & -- & $<2$ \\
\hline PP-3 & & NWQL & 20010405 & 0945 & 1.03 & 208 & 8.0 & 1.5 & 84 & 25.6 & 4.90 & 2 & 61 & 2.00 \\
\hline PP-3 & $\mathrm{R}$ & NWQL & 20010405 & 0950 & 1.03 & 208 & 8.0 & 1.5 & 84 & 25.7 & 4.90 & -- & -- & 1.90 \\
\hline PP-3 & & NWQL & 20010510 & 1315 & 8.88 & 173 & 7.5 & 7.5 & 66 & 20.0 & 4.04 & 1 & 86 & E1.71 \\
\hline PP-3 & & NWQL & 20010514 & 1220 & 22.4 & 109 & 7.7 & 9.5 & 43 & 13.0 & 2.59 & 8 & 76 & 2.29 \\
\hline PP-3 & & Boulder & 20010726 & 0600 & 5.66 & 244 & 7.0 & 10.5 & 91 & 27.8 & 5.25 & -- & -- & 1.97 \\
\hline PP-3 & $\mathrm{R}$ & NWQL & 20010726 & 0605 & 5.66 & 244 & 7.0 & 10.5 & -- & -- & -- & -- & -- & 2.46 \\
\hline PP-3a & & Boulder & 20010726 & 0608 & 13.3 & 222 & 7.8 & 9.5 & 92 & 27.4 & 5.72 & -- & -- & 1.67 \\
\hline PP-3b & & Boulder & 20010726 & 0645 & 12.2 & 225 & 7.9 & 9.5 & 92 & 27.4 & 5.74 & -- & -- & 1.95 \\
\hline PP-3c & & Boulder & 20010727 & 0730 & 12.7 & 243 & 8.0 & 11.0 & 92 & 27.4 & 5.78 & -- & -- & 1.99 \\
\hline PP-3d & & Boulder & 20010727 & 0910 & 14.8 & 226 & 7.8 & 9.5 & 86 & 25.4 & 5.41 & -- & -- & 4.92 \\
\hline PP-4 & & NWQL & 20010510 & 1500 & 25.9 & 190 & 8.0 & 10.0 & 67 & 19.7 & 4.20 & 8 & 73 & 6.12 \\
\hline PP-4 & & NWQL & 20010514 & 1310 & 50.9 & 137 & 7.9 & 11.5 & 48 & 14.3 & 2.91 & 33 & 60 & 7.91 \\
\hline PP-4 & & Boulder & 20010726 & 0715 & 15.4 & 247 & 8.0 & 11.0 & 86 & 25.5 & 5.49 & -- & -- & 4.53 \\
\hline PP-4 & $\mathrm{R}$ & Boulder & 20010726 & 0720 & 15.4 & -- & -- & -- & 87 & 25.7 & 5.48 & -- & -- & 4.80 \\
\hline PP-4 & & Boulder & 20010727 & 0930 & 14.8 & 243 & 8.0 & 12.0 & 86 & 25.4 & 5.41 & -- & -- & 4.82 \\
\hline PP-4a & & Boulder & 20010726 & 0525 & 17.3 & 256 & 7.9 & 11.9 & 90 & 26.5 & 5.79 & -- & -- & 4.80 \\
\hline PP-4b & & Boulder & 20010726 & 0600 & 17.2 & 256 & 7.9 & 12.0 & 91 & 26.7 & 5.99 & -- & -- & 4.98 \\
\hline PP-5 & & NWQL & 20010405 & 0900 & 14 & 313 & 7.7 & 0.0 & 114 & 33.1 & 7.66 & 5 & 75 & 3.94 \\
\hline PP-5 & & NWQL & 20010510 & 1345 & 49 & 190 & 8.2 & 10.5 & 69 & 20.3 & 4.47 & 16 & 55 & 5.41 \\
\hline PP-5 & $\mathrm{R}$ & NWQL & 20010510 & 1350 & 49 & 190 & 8.2 & 10.5 & 69 & 20.2 & 4.47 & 13 & 65 & 5.32 \\
\hline PP-5 & & NWQL & 20010514 & 1415 & 84 & 145 & 8.0 & 13.0 & 53 & 15.7 & 3.31 & 48 & 54 & 8.77 \\
\hline
\end{tabular}


Table 2. Water-quality data for routine and synoptic water samples collected from streams in the upper Prickly Pear Creek watershed, Montana, 2001 (Continued).

\begin{tabular}{|c|c|c|c|c|c|c|c|c|c|c|c|c|c|c|}
\hline $\begin{array}{c}\text { Site } \\
\text { number }\end{array}$ & $\begin{array}{c}\text { QA } \\
\text { sample } \\
\text { type }\end{array}$ & $\begin{array}{c}\text { Labora- } \\
\text { tory }\end{array}$ & Date & Time & $\begin{array}{c}\text { Arsenic, } \\
\text { dis- } \\
\text { solved } \\
(\mu \mathrm{g} / \mathrm{L})\end{array}$ & $\begin{array}{l}\text { Cad- } \\
\text { mium, } \\
\text { total } \\
\text { recov- } \\
\text { erable } \\
(\mu \mathrm{g} / \mathrm{L})\end{array}$ & $\begin{array}{c}\text { Cad- } \\
\text { mium, } \\
\text { dis- } \\
\text { solved } \\
(\mu \mathrm{g} / \mathrm{L})\end{array}$ & $\begin{array}{l}\text { Cop- } \\
\text { per, } \\
\text { total } \\
\text { recov- } \\
\text { erable } \\
(\mu \mathrm{g} / \mathrm{L})\end{array}$ & $\begin{array}{c}\text { Cop- } \\
\text { per, dis- } \\
\text { solved } \\
(\mu \mathrm{g} / \mathrm{L})\end{array}$ & $\begin{array}{l}\text { Lead, } \\
\text { total } \\
\text { recov- } \\
\text { erable } \\
(\mu \mathrm{g} / \mathrm{L})\end{array}$ & $\begin{array}{c}\text { Lead, } \\
\text { dis- } \\
\text { solved } \\
(\mu \mathrm{g} / \mathrm{L})\end{array}$ & $\begin{array}{c}\text { Molyb- } \\
\text { denum, } \\
\text { total } \\
\text { recov- } \\
\text { erable } \\
(\mu \mathrm{g} / \mathrm{L})\end{array}$ & $\begin{array}{l}\text { Zinc, } \\
\text { total } \\
\text { recov- } \\
\text { erable } \\
(\mu \mathrm{g} / \mathrm{L})\end{array}$ & $\begin{array}{c}\text { Zinc, dis- } \\
\text { solved } \\
(\mu \mathrm{g} / \mathrm{L})\end{array}$ \\
\hline L-6 & & NWQL & 20010510 & 1000 & 0.374 & $<0.04$ & $<0.04$ & 2.63 & 2.39 & $<1$ & $<0.08$ & -- & 1.30 & 1.22 \\
\hline L-7 & & NWQL & 20010510 & 1100 & 0.913 & 0.181 & 0.113 & 2.55 & 2.20 & $<1$ & 0.099 & -- & 129 & 120 \\
\hline L-7 & $\mathrm{R}$ & NWQL & 20010510 & 1105 & 0.937 & 0.158 & 0.118 & 2.47 & 2.15 & $<1$ & 0.098 & -- & 128 & 118 \\
\hline L-8 & & NWQL & 20010510 & 1045 & 0.376 & 0.049 & 0.037 & 6.99 & 5.47 & $<1$ & E0.045 & 10.2 & 5.0 & 4.14 \\
\hline L-9 & & NWQL & 20010510 & 1215 & 0.675 & 0.099 & 0.063 & 3.78 & 3.06 & $<1$ & E0.055 & -- & 58.0 & 49.8 \\
\hline L-9 & & Boulder & 20010726 & 1520 & 0.565 & 0.077 & $<0.05$ & 2.50 & 2.54 & $<0.1$ & 0.228 & -- & 21.5 & 18.7 \\
\hline L-9 & B & Boulder & 20010726 & 1525 & $<0.1$ & $<0.05$ & $<0.05$ & $<0.5$ & $<0.5$ & $<0.1$ & $<0.1$ & -- & 2.3 & 2.4 \\
\hline PP-1 & & NWQL & 20010510 & 0915 & 0.769 & E0.023 & E0.028 & 1.41 & 1.39 & $<1$ & E0.060 & -- & 2.28 & 1.92 \\
\hline PP-2 & & NWQL & 20010510 & 1040 & 0.646 & 0.048 & 0.042 & 1.45 & 1.35 & $<1$ & 0.160 & -- & 5.96 & 5.21 \\
\hline PP-2 & & NWQL & 20010514 & 1130 & 1.22 & 0.089 & E0.024 & 3.15 & 1.80 & 1.88 & 0.114 & -- & 10.1 & 4.96 \\
\hline PP-2 & & Boulder & 20010726 & 0815 & 0.227 & $<0.05$ & $<0.05$ & 1.69 & 1.05 & 0.957 & $<0.1$ & -- & 9.60 & 6.1 \\
\hline PP-2a & & Boulder & 20010726 & 0740 & 0.310 & 0.114 & 0.108 & 2.08 & 0.964 & 0.864 & $<0.1$ & -- & 8.70 & 6.6 \\
\hline PP-2a & $\mathrm{R}$ & Boulder & 20010726 & 0741 & 0.395 & 0.074 & 0.094 & 2.02 & 0.879 & 0.565 & $<0.1$ & -- & 8.50 & 6.6 \\
\hline PP-2a & $\mathrm{R}$ & NWQL & 20010726 & 0741 & -- & 0.066 & -- & 3.29 & -- & $<1$ & -- & -- & 13.2 & -- \\
\hline PP-3 & & NWQL & 20010405 & 0945 & 1.41 & 0.254 & 0.248 & 1.86 & 1.69 & $<1$ & E0.053 & -- & 125 & 130 \\
\hline PP-3 & $\mathrm{R}$ & NWQL & 20010405 & 0950 & 1.43 & 0.245 & 0.259 & 1.91 & 1.76 & $<1$ & E0.059 & -- & 127 & 132 \\
\hline PP-3 & & NWQL & 20010510 & 1315 & 1.73 & 0.379 & 0.380 & 3.74 & 2.85 & $<1$ & 0.108 & -- & 173 & 196 \\
\hline PP-3 & & NWQL & 20010514 & 1220 & 1.69 & 0.300 & 0.176 & 4.96 & 2.70 & 6.10 & 0.261 & -- & 100 & 85.0 \\
\hline PP-3 & & Boulder & 20010726 & 0600 & 1.73 & 0.685 & 0.768 & 2.60 & 2.56 & 1.79 & 0.197 & -- & 249 & 243 \\
\hline PP-3 & $\mathrm{R}$ & NWQL & 20010726 & 0605 & -- & 0.461 & -- & 2.86 & -- & 1.17 & -- & -- & 235 & -- \\
\hline PP-3a & & Boulder & 20010726 & 0608 & 1.59 & 0.561 & 0.659 & 1.20 & 1.91 & $<0.1$ & $<0.1$ & -- & 89.7 & 88.8 \\
\hline PP-3b & & Boulder & 20010726 & 0645 & 1.68 & 0.632 & 0.520 & 2.10 & 2.45 & 1.57 & $<0.1$ & -- & 192 & 183 \\
\hline PP-3c & & Boulder & 20010727 & 0730 & 1.79 & 0.644 & 0.682 & 2.87 & 2.24 & 1.58 & $<0.1$ & -- & 193 & 184 \\
\hline PP-3d & & Boulder & 20010727 & 0910 & 4.25 & 0.473 & 0.429 & 3.42 & 2.25 & 3.32 & 0.157 & -- & 138 & 128 \\
\hline PP-4 & & NWQL & 20010510 & 1500 & 4.33 & 0.301 & 0.181 & 3.37 & 2.08 & 4.17 & 0.253 & -- & 82.9 & 69.6 \\
\hline PP-4 & & NWQL & 20010514 & 1310 & 3.80 & 0.453 & 0.105 & 7.74 & 2.68 & 19.2 & 0.809 & -- & 101 & 54.1 \\
\hline PP-4 & & Boulder & 20010726 & 0715 & 3.65 & 0.563 & 0.496 & 2.96 & 2.52 & 2.23 & $<0.1$ & -- & 164 & 154 \\
\hline PP-4 & $\mathrm{R}$ & Boulder & 20010726 & 0720 & 3.74 & 0.586 & 0.516 & 2.99 & 2.27 & 2.39 & 0.346 & -- & 163 & 151 \\
\hline PP-4 & & Boulder & 20010727 & 0930 & 4.34 & 0.479 & 0.430 & 2.68 & 2.63 & 2.08 & 0.328 & -- & 130 & 122 \\
\hline PP-4a & & Boulder & 20010726 & 0525 & 3.74 & 0.538 & 0.463 & 2.71 & 2.59 & 1.82 & 0.181 & -- & 156 & 146 \\
\hline $\mathrm{PP}-4 \mathrm{~b}$ & & Boulder & 20010726 & 0600 & 4.00 & 0.254 & 0.254 & 3.06 & 2.45 & 2.73 & 0.446 & -- & 122 & 110 \\
\hline PP-5 & & NWQL & 20010405 & 0900 & 2.71 & 0.224 & 0.180 & 2.61 & 1.99 & 1.90 & 0.133 & -- & 81.3 & 75.7 \\
\hline PP-5 & & NWQL & 20010510 & 1345 & 3.78 & 0.258 & 0.093 & 4.09 & 2.55 & 6.86 & 0.291 & -- & 61.0 & 34.6 \\
\hline PP-5 & $\mathrm{R}$ & NWQL & 20010510 & 1350 & 3.74 & 0.274 & 0.093 & 4.11 & 2.53 & 5.58 & 0.303 & -- & 62.0 & 34.8 \\
\hline PP-5 & & NWQL & 20010514 & 1415 & 4.01 & 0.540 & 0.067 & 9.00 & 2.77 & 19.9 & 0.718 & -- & 106 & 35.7 \\
\hline
\end{tabular}


Table 2. Water-quality data for routine and synoptic water samples collected from streams in the upper Prickly Pear Creek watershed, Montana, 2001 (Continued).

\begin{tabular}{|c|c|c|c|c|c|c|c|c|c|c|c|c|c|c|}
\hline Site number & $\begin{array}{c}\text { QA } \\
\text { sample } \\
\text { type }\end{array}$ & $\begin{array}{c}\text { Lab- } \\
\text { oratory }\end{array}$ & Date & Time & $\begin{array}{c}\text { Stream- } \\
\text { flow } \\
\left(\mathbf{f t}^{3} / \mathbf{s}\right)\end{array}$ & $\begin{array}{c}\text { Specific } \\
\text { con- } \\
\text { ductance, } \\
\text { field }(\mu \mathrm{S} / \mathrm{cm})\end{array}$ & $\begin{array}{l}\text { pH, } \\
\text { field } \\
\text { (su) }\end{array}$ & $\begin{array}{c}\text { Temp- } \\
\text { erature, } \\
\text { water } \\
\left({ }^{\circ} \mathrm{C}\right)\end{array}$ & $\begin{array}{c}\text { Hard- } \\
\text { ness } \\
(\mathrm{mg} / \mathrm{L})\end{array}$ & $\begin{array}{c}\text { Calcium, } \\
\text { dissolved } \\
(\mathrm{mg} / \mathrm{L})\end{array}$ & $\begin{array}{l}\text { Magne- } \\
\text { sium, } \\
\text { dissolved } \\
(\mathrm{mg} / \mathrm{L})\end{array}$ & $\begin{array}{c}\text { Sediment, } \\
\text { suspended } \\
(\mathrm{mg} / \mathrm{L})\end{array}$ & $\begin{array}{l}\text { Sediment, } \\
\text { suspended, } \\
\text { diameter } \\
\text { (percent } \\
\text { finer than } \\
\text { 0.062 mm) }\end{array}$ & $\begin{array}{l}\text { Arsenic, } \\
\text { total recov- } \\
\text { erable } \\
(\mu \mathrm{g} / \mathrm{L})\end{array}$ \\
\hline PP-5 & & NWQL & 20010516 & 1045 & 73 & 152 & 7.6 & 7.5 & 57 & 16.9 & 3.58 & 31 & 41 & 6.54 \\
\hline PP-5 & & Boulder & 20010726 & 0700 & 17.7 & 266 & 8.1 & 12.7 & 94 & 27.5 & 6.17 & -- & -- & 5.01 \\
\hline PP-5 & B & Boulder & 20010726 & 0705 & -- & -- & -- & -- & -- & $<0.05$ & $<0.06$ & -- & -- & $<0.1$ \\
\hline PP-5 & & NWQL & 20010822 & 0945 & 8.6 & 239 & 8.2 & 12.5 & 104 & 30.1 & 7.03 & 2 & 83 & 5.82 \\
\hline S-1 & & NWQL & 20010511 & 1620 & 0.02 & 125 & 8.1 & 11.0 & 55 & 16.0 & 3.57 & 6 & 62 & 5.39 \\
\hline S-1a & & NWQL & 20010511 & 1815 & 0.17 & 275 & 7.1 & 6.0 & 111 & 34.1 & 6.30 & 57 & 85 & 23.3 \\
\hline S-3 & & NWQL & 20010511 & 1115 & 2.2 & 676 & 7.8 & 8.0 & 307 & 96.9 & 15.7 & -- & -- & 4.77 \\
\hline S-4 & & Boulder & 20010726 & 1030 & 0.75 & 627 & 7.8 & 12.5 & 324 & 103 & 16.4 & -- & -- & 5.01 \\
\hline S-4 & $\mathrm{R}$ & NWQL & 20010726 & 1035 & 0.75 & 627 & 7.8 & 12.5 & -- & -- & -- & -- & -- & 6.96 \\
\hline WS-1 & & NWQL & 20010511 & 1000 & 0.16 & 98 & 7.7 & 3.0 & 39 & 11.8 & 2.35 & 9 & 72 & E1.49 \\
\hline WS-2 & & NWQL & 20010509 & 1120 & 1.47 & 150 & 7.7 & 4.5 & 60 & 17.3 & 4.15 & 8 & 62 & 25.1 \\
\hline WS-2 & & NWQL & 20010627 & 1045 & 0.75 & 192 & 7.9 & 10.5 & 67 & 18.7 & 4.90 & -- & -- & 15.6 \\
\hline WS-2a & & NWQL & 20010509 & 1300 & 1.14 & 152 & 7.8 & 6.0 & 61 & 17.4 & 4.15 & 23 & 81 & 64.3 \\
\hline WS-3 & & NWQL & 20010405 & 1130 & 1.05 & 208 & 8.0 & 0.0 & 91 & 26.0 & 6.32 & 21 & 65 & 74.3 \\
\hline WS-3 & & NWQL & 20010509 & 1015 & 1.84 & 151 & 7.8 & 3.5 & 61 & 17.5 & 4.17 & 7 & 90 & 34.5 \\
\hline WS-3 & & NWQL & 20010627 & 0905 & 1.06 & 185 & 8.0 & 10.5 & 66 & 18.9 & 4.54 & -- & -- & 33.7 \\
\hline WS-4 & & NWQL & 20010509 & 0930 & 1.33 & 110 & 8.0 & 4.5 & 41 & 12.1 & 2.72 & 5 & 87 & E1.51 \\
\hline WS-5 & & NWQL & 20010509 & 0820 & 5.36 & 67 & 7.7 & 3.0 & 26 & 8.07 & 1.41 & 2 & 60 & E1.21 \\
\hline WS-7 & & NWQL & 20010509 & 0710 & 10.3 & 103 & 7.8 & 3.5 & 41 & 12.1 & 2.55 & 7 & 75 & 12.5 \\
\hline WS-8 & & NWQL & 20010510 & 1410 & 8.88 & 168 & 7.9 & 11.5 & 46 & 13.5 & 2.97 & 5 & 83 & 11.0 \\
\hline WS-8 & & Boulder & 20010726 & 1210 & 2.89 & 275 & 7.8 & 19.5 & 60 & 17.4 & 3.95 & -- & -- & 14.2 \\
\hline WS- 8 & & Boulder & 20010727 & 0650 & 3.1 & 308 & 7.8 & 16.0 & 59 & 17.1 & 3.94 & -- & -- & 15.3 \\
\hline
\end{tabular}


Table 2. Water-quality data for routine and synoptic water samples collected from streams in the upper Prickly Pear Creek watershed, Montana, 2001 (Continued)

\begin{tabular}{|c|c|c|c|c|c|c|c|c|c|c|c|c|c|c|}
\hline $\begin{array}{c}\text { Site } \\
\text { number }\end{array}$ & $\begin{array}{c}\text { QA } \\
\text { sample } \\
\text { type }\end{array}$ & $\begin{array}{l}\text { Labora- } \\
\text { tory }\end{array}$ & Date & Time & $\begin{array}{c}\text { Arsenic, } \\
\text { dis- } \\
\text { solved } \\
(\mu \mathrm{g} / \mathrm{L})\end{array}$ & $\begin{array}{c}\text { Cad- } \\
\text { mium, } \\
\text { total } \\
\text { recov- } \\
\text { erable } \\
(\mu \mathrm{g} / \mathrm{L})\end{array}$ & $\begin{array}{c}\text { Cad- } \\
\text { mium, } \\
\text { dis- } \\
\text { solved } \\
(\mu \mathrm{g} / \mathrm{L})\end{array}$ & $\begin{array}{l}\text { Cop- } \\
\text { per, } \\
\text { total } \\
\text { recov- } \\
\text { erable } \\
(\mu \mathrm{g} / \mathrm{L})\end{array}$ & $\begin{array}{c}\text { Cop- } \\
\text { per, dis- } \\
\text { solved } \\
(\mu \mathrm{g} / \mathrm{L})\end{array}$ & $\begin{array}{c}\text { Lead, } \\
\text { total } \\
\text { recov- } \\
\text { erable } \\
(\mu \mathrm{g} / \mathrm{L})\end{array}$ & $\begin{array}{c}\text { Lead, } \\
\text { dis- } \\
\text { solved } \\
(\mu \mathrm{g} / \mathrm{L})\end{array}$ & $\begin{array}{l}\text { Molyb- } \\
\text { denum, } \\
\text { total } \\
\text { recov- } \\
\text { erable } \\
(\mu \mathrm{g} / \mathrm{L})\end{array}$ & $\begin{array}{l}\text { Zinc, } \\
\text { total } \\
\text { recov- } \\
\text { erable } \\
(\mu \mathrm{g} / \mathrm{L})\end{array}$ & $\begin{array}{c}\text { Zinc, dis- } \\
\text { solved } \\
(\mu \mathrm{g} / \mathrm{L})\end{array}$ \\
\hline PP-5 & & NWQL & 20010516 & 1045 & -- & 0.287 & -- & 5.88 & -- & 11.4 & -- & -- & 89.5 & -- \\
\hline PP-5 & & Boulder & 20010726 & 0700 & 4.24 & 0.241 & 0.159 & 3.60 & 2.31 & 2.52 & 0.764 & -- & 71.5 & 62.5 \\
\hline PP-5 & B & Boulder & 20010726 & 0705 & $<0.1$ & $<0.05$ & $<0.05$ & $<0.5$ & $<0.5$ & $<0.1$ & $<0.1$ & -- & $<1$ & $<1$ \\
\hline PP-5 & & NWQL & 20010822 & 0945 & -- & 0.112 & -- & 2.12 & -- & 2.4 & -- & -- & 44.6 & -- \\
\hline S-1 & & NWQL & 20010511 & 1620 & 5.73 & $<0.04$ & $<0.04$ & E0.307 & E0.217 & $<1$ & $<0.08$ & -- & $<1$ & $<1$ \\
\hline S-1a & & NWQL & 20010511 & 1815 & 3.20 & 19.3 & 18.2 & 924 & 262 & 147 & 1.70 & -- & 2,330 & 2,290 \\
\hline S-3 & & NWQL & 20010511 & 1115 & 3.34 & 1.35 & 1.14 & 2.90 & 2.24 & 3.88 & 0.110 & 1.41 & 78.6 & 84.4 \\
\hline S-4 & & Boulder & 20010726 & 1030 & 5.01 & 1.30 & 1.30 & 4.04 & 3.23 & 8.08 & 0.446 & -- & 154 & 139 \\
\hline S-4 & $\mathrm{R}$ & NWQL & 20010726 & 1035 & -- & 1.02 & -- & 4.03 & -- & 7.94 & -- & -- & 155 & -- \\
\hline WS-1 & & NWQL & 20010511 & 1000 & 1.03 & $<0.04$ & $<0.04$ & 1.03 & 0.826 & $<1$ & 0.351 & -- & 1.48 & $<1$ \\
\hline WS-2 & & NWQL & 20010509 & 1120 & 10.3 & 2.95 & 2.59 & 4.37 & 1.73 & 5.46 & 0.308 & -- & 590 & 586 \\
\hline WS-2 & & NWQL & 20010627 & 1045 & 9.72 & 1.34 & 1.16 & 2.89 & 1.73 & $<1$ & E0.052 & -- & 302 & 240 \\
\hline WS-2a & & NWQL & 20010509 & 1300 & 13.0 & 2.84 & 2.19 & 6.32 & 2.84 & 14.5 & 0.268 & -- & 579 & 515 \\
\hline WS-3 & & NWQL & 20010405 & 1130 & 30.9 & 0.805 & 0.349 & 5.28 & 1.70 & 16.5 & E0.053 & -- & 224 & 145 \\
\hline WS-3 & & NWQL & 20010509 & 1015 & 20.0 & 1.63 & 1.39 & 4.33 & 2.96 & 5.62 & 0.185 & -- & 468 & 455 \\
\hline WS-3 & & NWQL & 20010627 & 0905 & 25.0 & 0.785 & 0.705 & 2.56 & 1.82 & 2.43 & 0.112 & -- & 250 & 241 \\
\hline WS-4 & & NWQL & 20010509 & 0930 & 1.04 & E0.018 & $<0.04$ & 2.75 & 2.05 & $<1$ & E0.045 & -- & 2.45 & $<1$ \\
\hline WS-5 & & NWQL & 20010509 & 0820 & 0.965 & $<0.04$ & $<0.04$ & 1.29 & 1.26 & $<1$ & E0.043 & -- & $<1$ & $<1$ \\
\hline WS-7 & & NWQL & 20010509 & 0710 & 6.84 & 0.303 & 0.171 & 2.48 & 1.77 & 2.09 & E0.074 & -- & 89.9 & 77.6 \\
\hline WS-8 & & NWQL & 20010510 & 1410 & 8.59 & 0.149 & 0.114 & 2.02 & 1.58 & 1.29 & 0.105 & -- & 50.0 & 44.6 \\
\hline WS-8 & & Boulder & 20010726 & 1210 & 13.0 & 0.102 & $<0.05$ & 0.102 & 1.89 & 1.49 & 0.455 & -- & 21.2 & 16.1 \\
\hline WS-8 & & Boulder & 20010727 & 0650 & 11.9 & 0.091 & 0.083 & 0.091 & 0.783 & 0.592 & 0.120 & -- & 20.8 & 18.5 \\
\hline
\end{tabular}


Table 3. Concentrations of additional constituents at synoptic water-quality sites in the upper Prickly Pear Creek watershed, Montana, July 26-27, 2001

[Samples analyzed by the U.S. Geological Survey laboratory, Boulder, Colo. Analytical data are reported to a maximum of three significant figures to provide sufficient resolution for detecting small differences between samples closely spaced in time or distance. Abbreviations: B, blank sample; mg/L, milligrams per liter; $\mu \mathrm{g} / \mathrm{L}$, micrograms per liter; $\mathrm{QA}$, quality assurance; R, replicate sample. Symbol: <, less than].

\begin{tabular}{|c|c|c|c|c|c|c|c|c|c|c|c|c|c|c|c|}
\hline $\begin{array}{c}\text { Site } \\
\text { number }\end{array}$ & $\begin{array}{c}\text { QA } \\
\text { sample } \\
\text { type }\end{array}$ & Date & Time & $\begin{array}{c}\text { Calcium, } \\
\text { total } \\
\text { recov- } \\
\text { erable } \\
(\mathrm{mg} / \mathrm{L})\end{array}$ & $\begin{array}{l}\text { Mag- } \\
\text { nesium, } \\
\text { total } \\
\text { recoverable } \\
(\mathrm{mg} / \mathrm{L})\end{array}$ & $\begin{array}{c}\text { Sodium, } \\
\text { total } \\
\text { recov- } \\
\text { erable } \\
(\mathrm{mg} / \mathrm{L})\end{array}$ & $\begin{array}{c}\text { Sodium, } \\
\text { dis- } \\
\text { solved } \\
(\mathrm{mg} / \mathrm{L})\end{array}$ & $\begin{array}{l}\text { Potassium, } \\
\text { total } \\
\text { recoverable } \\
(\mathrm{mg} / \mathrm{L})\end{array}$ & $\begin{array}{l}\text { Potas- } \\
\text { sium, } \\
\text { dis- } \\
\text { solved } \\
(\mathrm{mg} / \mathrm{L})\end{array}$ & $\begin{array}{c}\text { Sulfate, } \\
\text { dis- } \\
\text { solved } \\
(\mathrm{mg} / \mathrm{L} \text { as } \\
\left.\mathrm{SO}_{4}\right)\end{array}$ & $\begin{array}{c}\text { Chloride, } \\
\text { dissolved } \\
(\mathrm{mg} / \mathrm{L})\end{array}$ & $\begin{array}{c}\text { Fluoride, } \\
\text { dissolved } \\
(\mathrm{mg} / \mathrm{L})\end{array}$ & $\begin{array}{c}\text { Silica, } \\
\text { total } \\
\text { recov- } \\
\text { erable } \\
(\mathrm{mg} / \mathrm{L} \text { as } \\
\left.\mathrm{SiO}_{2}\right) \\
\end{array}$ & 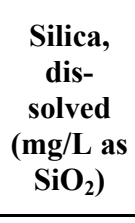 & $\begin{array}{c}\text { Nitrate, } \\
\text { dis- } \\
\text { solved } \\
(\mathrm{mg} / \mathrm{L} \text { as } \\
\left.\mathrm{NO}_{3}\right)\end{array}$ \\
\hline B-1 & & 20010726 & 0945 & 53.6 & 12.2 & 14.2 & 14.2 & 3.34 & 3.25 & 85.4 & 10.1 & 0.41 & 31.6 & 31.6 & 0.73 \\
\hline C-6 & & 20010726 & 1430 & 33.1 & 7.87 & 9.11 & 8.64 & 1.35 & 2.37 & 39.6 & 1.80 & $<0.4$ & 18.8 & 17.7 & 2.05 \\
\hline D-1 & & 20010726 & 1120 & 11.5 & 2.37 & 4.88 & 4.75 & 1.11 & 1.07 & 9.38 & 0.893 & $<0.4$ & 20.1 & 19.5 & 2.22 \\
\hline L-9 & & 20010726 & 1520 & 17.8 & 3.87 & 6.32 & 6.25 & 1.73 & 1.72 & 21.8 & 1.52 & $<0.4$ & 19.6 & 18.9 & 2.08 \\
\hline L-9 & B & 20010726 & 1525 & $<0.05$ & $<0.06$ & $<0.04$ & $<0.04$ & 0.002 & 0.016 & $<0.8$ & $<0.4$ & $<0.4$ & $<0.01$ & $<0.01$ & $<0.5$ \\
\hline PP-2 & & 20010726 & 0815 & 12.2 & 2.04 & 3.31 & 3.20 & 0.892 & 0.872 & 8.70 & 0.782 & $<0.4$ & 16.5 & 15.7 & 0.84 \\
\hline PP-2a & & 20010726 & 0740 & 14.1 & 2.55 & 3.75 & 3.63 & 1.01 & 0.987 & 12.3 & 1.10 & $<0.4$ & 17.2 & 16.6 & 0.65 \\
\hline PP-2a & $\mathrm{R}$ & 20010726 & 0741 & 14.2 & 2.55 & 3.80 & 3.64 & 1.01 & 1.00 & 12.4 & 1.11 & $<0.4$ & 17.4 & 16.6 & 0.68 \\
\hline PP-3 & & 20010726 & 0600 & 27.9 & 5.43 & 6.43 & 6.26 & 1.60 & 1.57 & 49.9 & 1.97 & $<0.4$ & 19.5 & 19.0 & $<0.5$ \\
\hline PP-3a & & 20010726 & 0608 & 26.9 & 5.76 & 6.88 & 6.85 & 1.74 & 1.71 & 49.8 & 2.24 & $<0.4$ & 21.4 & 21.3 & 0.72 \\
\hline PP-3b & & 20010726 & 0645 & 27.1 & 5.78 & 6.77 & 6.74 & 1.69 & 1.68 & 48.9 & 2.55 & $<0.4$ & 20.6 & 19.8 & 0.72 \\
\hline PP-3c & & 20010727 & 0730 & 27.6 & 5.93 & 7.07 & 6.83 & 1.78 & 1.72 & 49.4 & 2.47 & $<0.4$ & 20.6 & 20.0 & 1.26 \\
\hline PP-3d & & 20010727 & 0910 & 25.7 & 5.59 & 15.2 & 14.6 & 2.15 & 2.05 & 44.0 & 2.71 & $<0.4$ & 22.3 & 21.3 & 0.51 \\
\hline PP-4 & & 20010726 & 0715 & 25.9 & 5.63 & 15.3 & 15.0 & 2.17 & 2.14 & 45.8 & 2.72 & $<0.4$ & 23.3 & 21.6 & 0.74 \\
\hline PP-4 & $\mathrm{R}$ & 20010726 & 0720 & 25.8 & 5.57 & 15.3 & 15.0 & 2.20 & 2.14 & 45.4 & 2.75 & $<0.4$ & 22.3 & 21.7 & 0.63 \\
\hline PP-4 & & 20010727 & 0930 & 25.5 & 5.47 & 15.3 & 15.2 & 2.18 & 2.12 & 45.1 & 2.67 & $<0.4$ & 22.1 & 21.5 & 0.59 \\
\hline PP-4a & & 20010726 & 0525 & 26.7 & 5.92 & 15.6 & 15.2 & 2.33 & 2.23 & 45.3 & 2.86 & $<0.4$ & 22.2 & 21.3 & 0.74 \\
\hline PP-4b & & 20010726 & 0600 & 26.6 & 6.06 & 15.3 & 15.0 & 2.39 & 2.32 & 43.3 & 3.01 & $<0.4$ & 22.0 & 21.1 & 0.72 \\
\hline PP-5 & & 20010726 & 0700 & 27.5 & 6.26 & 15.5 & 15.3 & 2.51 & 2.42 & 43.3 & 3.43 & $<0.4$ & 21.6 & 20.8 & 0.66 \\
\hline PP-5 & B & 20010726 & 0705 & $<0.05$ & $<0.06$ & $<0.04$ & $<0.04$ & $<0.002$ & $<0.002$ & $<0.8$ & $<0.4$ & $<0.4$ & $<0.01$ & $<0.01$ & $<0.5$ \\
\hline S-4 & & 20010726 & 1030 & 106 & 16.8 & 17.3 & 16.7 & 2.92 & 2.67 & 270 & 4.88 & 0.12 & 21.2 & 20.3 & 2.09 \\
\hline WS-8 & & 20010726 & 1210 & 17.6 & 4.00 & 44.0 & 43.5 & 3.56 & 3.41 & 32.1 & 2.86 & 1.24 & 26.8 & 26.8 & $<0.5$ \\
\hline WS-8 & & 20010727 & 0650 & 17.4 & 4.00 & 44.1 & 43.2 & 3.61 & 3.45 & 31.9 & 2.92 & 1.28 & 28.4 & 27.0 & $<0.5$ \\
\hline
\end{tabular}


Table 3. Concentrations of additional constituents at synoptic water-quality sites in the upper Prickly Pear Creek watershed, Montana, July 26-27, 2001 (Continued).

\begin{tabular}{|c|c|c|c|c|c|c|c|c|c|c|c|c|c|c|c|c|c|}
\hline $\begin{array}{c}\text { Site } \\
\text { number }\end{array}$ & $\begin{array}{c}\text { QA } \\
\text { sample } \\
\text { type }\end{array}$ & Date & Time & $\begin{array}{l}\text { Alumi- } \\
\text { num, } \\
\text { total } \\
\text { recov- } \\
\text { erable } \\
(\mu \mathrm{g} / \mathrm{L}) \\
\end{array}$ & $\begin{array}{c}\text { Alumi- } \\
\text { num, } \\
\text { dis- } \\
\text { solved } \\
(\mu \mathrm{g} / \mathrm{L})\end{array}$ & $\begin{array}{c}\text { Barium, } \\
\text { total } \\
\text { recov- } \\
\text { erable } \\
(\mu \mathrm{g} / \mathrm{L})\end{array}$ & $\begin{array}{c}\text { Barium, } \\
\text { dis- } \\
\text { solved } \\
(\mu \mathrm{g} / \mathrm{L})\end{array}$ & $\begin{array}{l}\text { Beryl- } \\
\text { lium, } \\
\text { total } \\
\text { recov- } \\
\text { erable } \\
(\mu \mathrm{g} / \mathrm{L}) \\
\end{array}$ & $\begin{array}{l}\text { Beryl- } \\
\text { lium, } \\
\text { dis- } \\
\text { solved } \\
(\mu \mathrm{g} / \mathrm{L})\end{array}$ & $\begin{array}{c}\text { Boron, } \\
\text { total } \\
\text { recov- } \\
\text { erable } \\
(\mu \mathrm{g} / \mathrm{L})\end{array}$ & $\begin{array}{c}\text { Boron, } \\
\text { dis- } \\
\text { solved } \\
(\mu \mathrm{g} / \mathrm{L})\end{array}$ & $\begin{array}{c}\text { Cobalt, } \\
\text { total } \\
\text { recov- } \\
\text { erable } \\
(\mu \mathrm{g} / \mathrm{L})\end{array}$ & $\begin{array}{c}\text { Cobalt, } \\
\text { dis- } \\
\text { solved } \\
(\mu \mathrm{g} / \mathrm{L})\end{array}$ & $\begin{array}{c}\text { Chro- } \\
\text { mium, } \\
\text { total } \\
\text { recov- } \\
\text { erable } \\
(\mu \mathrm{g} / \mathrm{L}) \\
\end{array}$ & $\begin{array}{c}\text { Chro- } \\
\text { mium, } \\
\text { dis- } \\
\text { solved } \\
(\mu \mathrm{g} / \mathrm{L})\end{array}$ & $\begin{array}{l}\text { Iron, } \\
\text { total } \\
\text { recov- } \\
\text { erable } \\
(\mu \mathrm{g} / \mathrm{L})\end{array}$ & $\begin{array}{c}\text { Iron, } \\
\text { dis- } \\
\text { solved } \\
(\mu \mathrm{g} / \mathrm{L})\end{array}$ \\
\hline B-1 & & 20010726 & 0945 & $<80$ & $<80$ & 23.1 & 21.1 & $<0.1$ & $<0.1$ & 29.1 & 32.2 & $<1$ & $<1$ & $<1$ & $<1$ & 614 & 75.1 \\
\hline C-6 & & 20010726 & 1430 & $<80$ & $<80$ & 34.9 & 33.0 & $<.1$ & $<.1$ & 23.8 & 24.0 & $<1$ & $<1$ & $<1$ & $<1$ & 47.1 & $<9$ \\
\hline D-1 & & 20010726 & 1120 & $<80$ & $<80$ & 14.5 & 14.1 & $<.1$ & $<.1$ & 18.6 & 19.6 & $<1$ & $<1$ & $<1$ & $<1$ & 85.8 & 41.2 \\
\hline L-9 & & 20010726 & 1520 & $<80$ & $<80$ & 26.1 & 25.5 & $<.1$ & $<.1$ & 19.9 & 17.7 & $<1$ & $<1$ & $<1$ & $<1$ & 148 & 76.8 \\
\hline L-9 & B & 20010726 & 1525 & $<80$ & $<80$ & $<0.5$ & $<0.5$ & $<.1$ & $<.1$ & 13.0 & 12 & $<1$ & $<1$ & $<1$ & $<1$ & $<9$ & $<9$ \\
\hline PP-2 & & 20010726 & 0815 & $<80$ & $<80$ & 7.30 & 6.70 & $<.1$ & $<.1$ & 23.4 & 23.0 & $<1$ & $<1$ & 1.1 & $<1$ & 74.4 & 12.0 \\
\hline PP-2a & & 20010726 & 0740 & $<80$ & $<80$ & 8.20 & 7.50 & $<.1$ & $<.1$ & 4.30 & 4.50 & $<1$ & $<1$ & $<1$ & $<1$ & 156 & 36.6 \\
\hline PP-2a & $\mathrm{R}$ & 20010726 & 0741 & $<80$ & $<80$ & 8.40 & 7.50 & $<.1$ & $<.1$ & 24.8 & 4.40 & $<1$ & $<1$ & $<1$ & $<1$ & 147 & 36.2 \\
\hline PP-3 & & 20010726 & 0600 & $<80$ & $<80$ & 14.9 & 14.7 & $<.1$ & $<.1$ & 7.00 & 7.30 & $<1$ & $<1$ & $<1$ & $<1$ & 36.1 & $<9$ \\
\hline PP-3a & & 20010726 & 0608 & $<80$ & $<80$ & 21.6 & 21.5 & $<.1$ & $<.1$ & 8.70 & 8.30 & $<1$ & $<1$ & $<1$ & $<1$ & $<9$ & $<9$ \\
\hline PP-3b & & 20010726 & 0645 & $<80$ & $<80$ & 22.2 & 21.9 & $<.1$ & $<.1$ & 8.80 & 8.50 & $<1$ & $<1$ & $<1$ & $<1$ & 66.6 & 11.5 \\
\hline PP-3c & & 20010727 & 0730 & $<80$ & $<80$ & 23.3 & 22.7 & $<.1$ & $<.1$ & 22.5 & 22.1 & 2.8 & $<1$ & $<1$ & $<1$ & 55.3 & 15.1 \\
\hline PP-3d & & 20010727 & 0910 & $<80$ & $<80$ & 24.5 & 23.7 & $<.1$ & $<.1$ & 32.2 & 31.7 & 2.2 & $<1$ & $<1$ & $<1$ & 112 & 22.8 \\
\hline PP-4 & & 20010726 & 0715 & $<80$ & $<80$ & 24.8 & 24.1 & $<.1$ & $<.1$ & 19.7 & 19.2 & $<1$ & $<1$ & $<1$ & $<1$ & 75.5 & 19.7 \\
\hline PP-4 & $\mathrm{R}$ & 20010726 & 0720 & $<80$ & $<80$ & 23.8 & 24.3 & $<.1$ & $<.1$ & 19.9 & 19.3 & $<1$ & $<1$ & $<1$ & $<1$ & 75.3 & 19.6 \\
\hline PP-4 & & 20010727 & 0930 & $<80$ & $<80$ & 24.0 & 23.4 & $<.1$ & $<.1$ & 31.2 & 32.4 & $<1$ & $<1$ & $<1$ & $<1$ & 83.3 & 23.5 \\
\hline PP-4a & & 20010726 & 0525 & $<80$ & $<80$ & 26.3 & 25.9 & $<.1$ & $<.1$ & 21.4 & 20.2 & $<1$ & $<1$ & $<1$ & $<1$ & 70.7 & 22.5 \\
\hline PP-4b & & 20010726 & 0600 & $<80$ & $<80$ & 28.0 & 27.3 & $<.1$ & $<.1$ & 20.9 & 19.9 & $<1$ & $<1$ & $<1$ & $<1$ & 151 & 59.3 \\
\hline PP-5 & & 20010726 & 0700 & $<80$ & $<80$ & 27.8 & 26.9 & $<.1$ & $<.1$ & 21.8 & 21.8 & $<1$ & $<1$ & $<1$ & $<1$ & 115 & 44.3 \\
\hline PP-5 & B & 20010726 & 0705 & $<80$ & $<80$ & $<0.5$ & $<0.5$ & $<.1$ & $<.1$ & $<3$ & $<3$ & $<1$ & $<1$ & $<1$ & $<1$ & $<9$ & $<9$ \\
\hline S-4 & & 20010726 & 1030 & $<80$ & $<80$ & 41.4 & 40.1 & 0.10 & $<.1$ & 28.0 & 29.4 & $<1$ & $<1$ & $<1$ & $<1$ & 53.8 & $<9$ \\
\hline WS-8 & & 20010726 & 1210 & 91.0 & $<80$ & 28.3 & 27.3 & 0.21 & 0.20 & 67.5 & 68.5 & $<1$ & $<1$ & $<1$ & $<1$ & 167 & 50.7 \\
\hline WS-8 & & 20010727 & 0650 & 84.3 & $<80$ & 28.2 & 27.2 & $<.14$ & 0.1 & 69.6 & 68.7 & $<1$ & $<1$ & $<1$ & $<1$ & 167 & 51.1 \\
\hline
\end{tabular}


Table 3. Concentrations of additional constituents at synoptic water-quality sites in the upper Prickly Pear Creek watershed, Montana, July 26-27, 2001 (Continued).

\begin{tabular}{|c|c|c|c|c|c|c|c|c|c|c|c|c|c|c|c|c|}
\hline $\begin{array}{c}\text { Site } \\
\text { number }\end{array}$ & $\begin{array}{c}\text { QA } \\
\text { sample } \\
\text { type }\end{array}$ & Date & Time & $\begin{array}{l}\text { Lead, } \\
\text { total } \\
\text { recov- } \\
\text { erable } \\
(\mu \mathrm{g} / \mathrm{L})\end{array}$ & $\begin{array}{c}\text { Lead, } \\
\text { dis- } \\
\text { solved } \\
(\mu \mathrm{g} / \mathrm{L})\end{array}$ & $\begin{array}{c}\text { Lithium, } \\
\text { total } \\
\text { recov- } \\
\text { erable } \\
(\mu \mathrm{g} / \mathrm{L})\end{array}$ & $\begin{array}{c}\text { Lithium, } \\
\text { dissolved } \\
(\mu \mathrm{g} / \mathrm{L})\end{array}$ & $\begin{array}{c}\text { Man- } \\
\text { ganese, } \\
\text { total } \\
\text { recov- } \\
\text { erable } \\
(\mu \mathrm{g} / \mathrm{L})\end{array}$ & $\begin{array}{c}\text { Nickel, } \\
\text { total } \\
\text { recov- } \\
\text { erable } \\
(\mu \mathrm{g} / \mathrm{L})\end{array}$ & $\begin{array}{l}\text { Nickel, } \\
\text { dissolve } \\
\text { d ( } \mu \mathrm{g} / \mathrm{L})\end{array}$ & $\begin{array}{c}\text { Seleniu } \\
\text { m, total } \\
\text { recov- } \\
\text { erable } \\
(\mu \mathrm{g} / \mathrm{L})\end{array}$ & $\begin{array}{c}\text { Seleniu } \\
\text { m, } \\
\text { dissolve } \\
\text { d }(\mu \mathrm{g} / \mathrm{L})\end{array}$ & $\begin{array}{c}\text { Strontium, } \\
\text { total } \\
\text { recov- } \\
\text { erable } \\
(\mu \mathrm{g} / \mathrm{L})\end{array}$ & $\begin{array}{l}\text { Stron- } \\
\text { tium, } \\
\text { dis- } \\
\text { solved } \\
(\mu \mathrm{g} / \mathrm{L})\end{array}$ & $\begin{array}{l}\text { Vana- } \\
\text { dium, } \\
\text { total } \\
\text { recov- } \\
\text { erable } \\
(\mu \mathrm{g} / \mathrm{L})\end{array}$ & $\begin{array}{c}\text { Vana- } \\
\text { dium, } \\
\text { dis- } \\
\text { solved } \\
(\mu \mathrm{g} / \mathrm{L})\end{array}$ \\
\hline B-1 & & 20010726 & 0945 & 0.170 & $<0.1$ & 8.1 & $<8$ & 116 & $<2$ & $<2$ & $<50$ & $<50$ & 331 & 327 & $<1$ & $<1$ \\
\hline C-6 & & 20010726 & 1430 & 0.529 & $<0.1$ & $<8$ & 8.3 & 7.4 & $<2$ & $<2$ & $<50$ & $<50$ & 247 & 234 & 1.1 & 1.0 \\
\hline D-1 & & 20010726 & 1120 & $<0.1$ & $<0.1$ & $<8$ & $<8$ & 4.1 & $<2$ & $<2$ & $<50$ & $<50$ & 97.4 & 95.7 & 1.6 & 1.8 \\
\hline L-9 & & 20010726 & 1520 & $<0.1$ & 0.228 & $<8$ & $<8$ & 24.4 & $<2$ & $<2$ & $<50$ & $<50$ & 154 & 150 & $<1$ & $<1$ \\
\hline L-9 & B & 20010726 & 1525 & $<0.1$ & $<0.1$ & $<8$ & $<8$ & $<1$ & $<2$ & $<2$ & $<50$ & $<50$ & $<1$ & $<1$ & $<1$ & $<1$ \\
\hline PP-2 & & 20010726 & 0815 & 0.957 & $<0.1$ & $<8$ & $<8$ & 3.8 & $<2$ & $<2$ & $<50$ & $<50$ & 84.8 & 83.7 & $<1$ & 1.1 \\
\hline PP-2a & & 20010726 & 0740 & 0.864 & $<0.1$ & $<8$ & $<8$ & 20.3 & $<2$ & $<2$ & $<50$ & $<50$ & 96.7 & 95.7 & $<1$ & $<1$ \\
\hline PP-2a & $\mathrm{R}$ & 20010726 & 0741 & 0.879 & $<0.1$ & $<8$ & $<8$ & 18.0 & $<2$ & $<2$ & $<50$ & $<50$ & 98.5 & 97.0 & 1.0 & $<1$ \\
\hline PP-3 & & 20010726 & 0600 & 1.79 & 0.197 & $<8$ & $<8$ & 19.1 & $<2$ & $<2$ & $<50$ & $<50$ & 223 & 223 & $<1$ & $<1$ \\
\hline PP-3a & & 20010726 & 0608 & $<0.1$ & $<0.1$ & $<8$ & $<8$ & $<1$ & $<2$ & $<2$ & $<50$ & $<50$ & 220 & 218 & $<1$ & 1.1 \\
\hline PP-3b & & 20010726 & 0645 & 1.57 & $<0.1$ & $<8$ & $<8$ & 22.4 & $<2$ & $<2$ & $<50$ & $<50$ & 213 & 216 & $<1$ & $<1$ \\
\hline PP-3c & & 20010727 & 0730 & 1.58 & $<0.1$ & $<8$ & $<8$ & 31.5 & $<2$ & $<2$ & $<50$ & $<50$ & 219 & 214 & $<1$ & 1.0 \\
\hline PP-3d & & 20010727 & 0910 & 3.32 & 0.157 & 22.4 & 21.2 & 35.3 & $<2$ & $<2$ & $<50$ & $<50$ & 245 & 239 & 1.0 & $<1$ \\
\hline PP-4 & & 20010726 & 0715 & 2.23 & $<0.1$ & 22.7 & 22.2 & 38.2 & $<2$ & $<2$ & $<50$ & $<50$ & 248 & 245 & $<1$ & 1.0 \\
\hline PP-4 & $\mathrm{R}$ & 20010726 & 0720 & 2.39 & 0.346 & 22.3 & 21.7 & 38.4 & 2.30 & $<2$ & $<50$ & $<50$ & 247 & 246 & $<1$ & $<1$ \\
\hline PP-4 & & 20010727 & 0930 & 2.08 & 0.328 & 22.8 & 21.7 & 33.1 & $<2$ & $<2$ & $<50$ & $<50$ & 246 & 240 & 1.0 & 1.2 \\
\hline PP-4a & & 20010726 & 0525 & 1.82 & 0.181 & 22.7 & 21.6 & 38.8 & $<2$ & $<2$ & $<50$ & $<50$ & 252 & 248 & $<1$ & $<1$ \\
\hline PP-4b & & 20010726 & 0600 & 2.73 & 0.446 & 22.2 & 21.1 & 65.7 & $<2$ & $<2$ & $<50$ & $<50$ & 252 & 246 & $<1$ & $<1$ \\
\hline PP-5 & & 20010726 & 0700 & 2.52 & 0.764 & 23.2 & 21.7 & 42.4 & $<2$ & $<2$ & $<50$ & $<50$ & 253 & 248 & $<1$ & $<1$ \\
\hline PP-5 & B & 20010726 & 0705 & $<0.1$ & $<0.1$ & $<8$ & $<8$ & $<1$ & $<2$ & $<2$ & $<50$ & $<50$ & $<1$ & $<1$ & $<1$ & $<1$ \\
\hline S-4 & & 20010726 & 1030 & 8.08 & 0.446 & 12.3 & 11.5 & 29.8 & $<2$ & $<2$ & $<50$ & $<50$ & 1,030 & 1,010 & $<1$ & $<1$ \\
\hline WS-8 & & 20010726 & 1210 & 1.49 & 0.455 & 91.0 & 85.7 & 19.9 & $<2$ & $<2$ & $<50$ & $<50$ & 332 & 324 & 1.3 & $<1$ \\
\hline WS-8 & & 20010727 & 0650 & 0.592 & 0.200 & 91.3 & 86.6 & 19.9 & $<2$ & $<2$ & $<50$ & $<50$ & 331 & 325 & 1.1 & 1.1 \\
\hline
\end{tabular}


Table 4. Water-quality data for diel investigations in Prickly Pear Creek at site PP-5, Montana, March-April 2001.

[Samples analyzed by the U.S. Geological Survey National Water Quality Laboratory, Denver, Colo. Analytical data are reported to a maximum of three significant figures to provide sufficient resolution for detecting small differences between samples closely spaced in time or distance. Similarly, field measurements from electronic recorders have not been rounded to illustrate the pattern of relative change between measurements. Abbreviations: $\mathrm{ft}^{3} / \mathrm{s}$, cubic feet per second; ${ }^{\circ} \mathrm{C}$, degrees Celsius; $\mathrm{mg} / \mathrm{L}$, micrograms per liter; $\mathrm{S} / \mathrm{cm}$, microsiemens per centimeterat $25^{\circ} \mathrm{C} ; \mathrm{mg} / \mathrm{L}$, milligrams per liter; su, standard units. Symbol: --, no data]

\begin{tabular}{|c|c|c|c|c|c|c|}
\hline Date and time & $\begin{array}{c}\text { Streamflow, } \\
\text { instantaneous } \\
\left(\mathrm{ft}^{3} / \mathbf{s}\right)\end{array}$ & $\begin{array}{c}\text { Specific } \\
\text { conductance, } \\
\text { field } \\
(\mu \mathrm{S} / \mathrm{cm})\end{array}$ & $\begin{array}{l}\text { pH, } \\
\text { field } \\
\text { (su) }\end{array}$ & $\begin{array}{c}\text { Temp- } \\
\text { erature, } \\
\text { water } \\
\left({ }^{\circ} \mathrm{C}\right) \\
\end{array}$ & $\begin{array}{c}\text { Dissolved } \\
\text { oxygen, } \\
\text { field } \\
(\mathrm{mg} / \mathrm{L}) \\
\end{array}$ & $\begin{array}{c}\text { Zinc, } \\
\text { dissolved } \\
(\mu \mathrm{g} / \mathrm{L})\end{array}$ \\
\hline \multicolumn{7}{|c|}{ March 13-14, 2001} \\
\hline $3 / 13 / 01 \quad 3: 00$ & 14.5 & -- & 7.90 & 2.90 & -- & 74.2 \\
\hline $3 / 13 / 01 \quad 4: 00$ & 14.5 & -- & 7.91 & 2.81 & -- & 76.5 \\
\hline $3 / 13 / 01 \quad 5: 00$ & 14.1 & -- & 7.91 & 2.74 & -- & 78.8 \\
\hline $3 / 13 / 01 \quad 6: 00$ & 14.1 & -- & 7.91 & 2.68 & -- & 81.1 \\
\hline $3 / 13 / 01 \quad 7: 00$ & 14.1 & -- & 7.91 & 2.64 & -- & 81.3 \\
\hline $3 / 13 / 01 \quad 8: 00$ & 14.1 & -- & 7.91 & 2.65 & -- & 82.8 \\
\hline $3 / 13 / 01 \quad 9: 00$ & 13.8 & -- & 7.91 & 2.86 & -- & 82.4 \\
\hline $3 / 13 / 01 \quad 10: 00$ & 13.4 & -- & 7.91 & 3.13 & -- & 81.3 \\
\hline $3 / 13 / 0111: 00$ & 13.8 & -- & 7.90 & 3.45 & -- & 78.2 \\
\hline $3 / 13 / 0112: 00$ & 13.8 & -- & 7.90 & 3.83 & -- & 74.9 \\
\hline $3 / 13 / 0113: 00$ & 13.8 & -- & 7.92 & 4.16 & -- & 70.8 \\
\hline $3 / 13 / 0114: 00$ & 14.1 & -- & 7.91 & 4.99 & -- & 65.8 \\
\hline $3 / 13 / 01 \quad 15: 00$ & 14.5 & -- & 7.93 & 5.91 & -- & 59.5 \\
\hline $3 / 13 / 01 \quad 16: 00$ & 15.3 & -- & 7.95 & 5.76 & -- & 53.5 \\
\hline $3 / 13 / 0117: 00$ & 15.6 & -- & 7.97 & 5.51 & -- & 48.1 \\
\hline $3 / 13 / 0118: 00$ & 16.0 & -- & 7.98 & 5.24 & -- & 43.7 \\
\hline $3 / 13 / 01 \quad 19: 00$ & 16.9 & -- & 7.99 & 4.98 & -- & 40.0 \\
\hline $3 / 13 / 0120: 00$ & 16.9 & -- & 7.98 & 4.73 & -- & 40.2 \\
\hline $3 / 13 / 0121: 00$ & 17.3 & -- & 7.97 & 4.34 & -- & 44.2 \\
\hline $3 / 13 / 0122: 00$ & 17.3 & -- & 7.95 & 3.97 & -- & 42.6 \\
\hline $3 / 13 / 0123: 00$ & 17.7 & -- & 7.93 & 3.41 & -- & 50.5 \\
\hline $3 / 14 / 01 \quad 0: 00$ & 17.7 & -- & 7.91 & 3.22 & -- & 54.8 \\
\hline $3 / 14 / 01 \quad 1: 00$ & 17.7 & -- & 7.90 & 2.91 & -- & 61.4 \\
\hline $3 / 14 / 01 \quad 2: 00$ & 17.3 & -- & 7.90 & 2.66 & -- & 69.0 \\
\hline $3 / 14 / 01 \quad 3: 00$ & 16.4 & -- & 7.89 & 2.42 & -- & 76.0 \\
\hline $3 / 14 / 01 \quad 4: 00$ & 16.0 & -- & 7.89 & 2.11 & -- & 80.0 \\
\hline $3 / 14 / 01 \quad 5: 00$ & 16.0 & -- & 7.89 & 1.84 & -- & 82.3 \\
\hline $3 / 14 / 01 \quad 6: 00$ & 15.6 & -- & 7.88 & 1.63 & -- & 85.8 \\
\hline $3 / 14 / 01 \quad 7: 00$ & 15.3 & -- & 7.89 & 1.51 & -- & 84.4 \\
\hline $3 / 14 / 01 \quad 8: 00$ & 15.3 & -- & 7.88 & 1.46 & -- & 85.5 \\
\hline $3 / 14 / 01 \quad 9: 00$ & 14.5 & -- & 7.89 & 1.39 & -- & 85.6 \\
\hline $3 / 14 / 0111: 00$ & 14.9 & -- & 7.89 & 0.80 & -- & 84.0 \\
\hline $3 / 14 / 0112: 00$ & 14.9 & -- & 7.86 & 1.36 & -- & 80.2 \\
\hline $3 / 14 / 0113: 00$ & 14.9 & -- & 7.87 & 1.85 & -- & 75.8 \\
\hline $3 / 14 / 0114: 00$ & 14.5 & -- & 7.88 & 2.56 & -- & 71.2 \\
\hline $3 / 14 / 0115: 00$ & 14.1 & -- & 7.92 & 3.25 & -- & 66.5 \\
\hline $3 / 14 / 0116: 00$ & 14.5 & -- & 7.94 & 3.56 & -- & 61.7 \\
\hline $3 / 14 / 0117: 00$ & 14.5 & -- & 7.97 & 3.69 & -- & 55.2 \\
\hline $3 / 14 / 0118: 00$ & 14.5 & -- & 7.98 & 3.66 & -- & 50.8 \\
\hline $3 / 14 / 0119: 00$ & 14.1 & -- & 7.98 & 3.39 & -- & 46.3 \\
\hline $3 / 14 / 0120: 00$ & 14.1 & -- & 7.97 & 3.05 & -- & 44.0 \\
\hline \multicolumn{7}{|c|}{ March 22-23, 2001} \\
\hline $3 / 22 / 0111: 00$ & 13.0 & -- & -- & -- & -- & -- \\
\hline $3 / 22 / 01 \quad 11: 20$ & -- & -- & 7.85 & 3.40 & -- & 68.4 \\
\hline
\end{tabular}


Table 4. Water-quality data for diel investigations in Prickly Pear Creek at site PP-5, Montana, March-April 2001 (Continued).

\begin{tabular}{|c|c|c|c|c|c|c|}
\hline Date and time & $\begin{array}{c}\text { Streamflow, } \\
\text { instantaneous } \\
\left(\mathrm{ft}^{3} / \mathbf{s}\right)\end{array}$ & $\begin{array}{c}\text { Specific } \\
\text { conductance, } \\
\text { field } \\
(\mu \mathrm{S} / \mathrm{cm}) \\
\end{array}$ & $\begin{array}{c}\text { pH, } \\
\text { field } \\
\text { (su) }\end{array}$ & $\begin{array}{c}\text { Temp- } \\
\text { erature, } \\
\text { water } \\
\left({ }^{0} \mathrm{C}\right) \\
\end{array}$ & $\begin{array}{l}\text { Dissolved } \\
\text { oxygen, } \\
\text { field } \\
(\mathrm{mg} / \mathrm{L}) \\
\end{array}$ & $\begin{array}{c}\text { Zinc, } \\
\text { dissolved } \\
(\mu \mathrm{g} / \mathrm{L})\end{array}$ \\
\hline \multicolumn{7}{|c|}{ March 22-23, 2001 (Continued) } \\
\hline $3 / 22 / 01 \quad 12: 00$ & 13.4 & -- & 7.84 & 4.24 & -- & 65.7 \\
\hline $3 / 22 / 01$ 13:00 & 16.0 & -- & 7.90 & 6.00 & -- & 57.9 \\
\hline $3 / 22 / 0114: 00$ & 17.7 & -- & 8.01 & 7.37 & -- & 50.9 \\
\hline $3 / 22 / 01$ 15:00 & 20.4 & -- & 8.08 & 8.18 & -- & 40.9 \\
\hline $3 / 22 / 01$ 16:00 & 24.2 & -- & 8.12 & 8.34 & -- & 31.4 \\
\hline $3 / 22 / 0117: 00$ & 24.8 & -- & 8.18 & 8.00 & -- & 24.8 \\
\hline $3 / 22 / 01$ 18:00 & 24.2 & -- & 8.24 & 7.81 & -- & 21.0 \\
\hline $3 / 22 / 0119: 00$ & 23.7 & -- & 8.20 & 7.20 & -- & 21.0 \\
\hline $3 / 22 / 0120: 00$ & 23.2 & -- & 8.11 & 6.40 & -- & 22.8 \\
\hline $3 / 22 / 0121: 00$ & 21.8 & -- & 8.02 & 5.35 & -- & 28.1 \\
\hline $3 / 22 / 0122: 00$ & 21.8 & -- & 7.96 & 4.57 & -- & 31.9 \\
\hline $3 / 22 / 0123: 00$ & 21.3 & -- & 7.93 & 4.04 & -- & 37.2 \\
\hline $3 / 23 / 01 \quad 0: 00$ & 20.4 & -- & 7.92 & 3.58 & -- & 41.6 \\
\hline $3 / 23 / 01 \quad 1: 00$ & 18.1 & -- & 7.91 & 3.12 & -- & 48.0 \\
\hline $3 / 23 / 01 \quad 2: 00$ & 17.3 & -- & 7.90 & 2.63 & -- & 53.0 \\
\hline $3 / 23 / 01 \quad 3: 00$ & 16.0 & -- & 7.89 & 2.13 & -- & 57.6 \\
\hline $3 / 23 / 01 \quad 7: 00$ & 14.9 & -- & 7.86 & 1.15 & -- & 70.9 \\
\hline $3 / 23 / 01 \quad 8: 00$ & 14.9 & -- & 7.86 & 1.06 & -- & 66.7 \\
\hline $3 / 23 / 01 \quad 9: 00$ & 14.5 & -- & 7.88 & 1.11 & -- & 67.8 \\
\hline $3 / 23 / 01 \quad 10: 00$ & 14.1 & -- & 7.89 & 1.42 & -- & 65.3 \\
\hline $3 / 23 / 01$ 11:00 & 13.8 & -- & 7.90 & 2.39 & -- & 62.2 \\
\hline $3 / 23 / 0112: 00$ & 14.1 & -- & 7.93 & 3.98 & -- & 56.5 \\
\hline $3 / 23 / 01$ 13:00 & 16.0 & -- & 7.97 & 5.42 & -- & 49.1 \\
\hline $3 / 23 / 0114: 00$ & 19.9 & -- & 8.02 & 6.56 & -- & 44.6 \\
\hline $3 / 23 / 01$ 15:00 & 21.3 & -- & 8.07 & 7.10 & -- & 35.4 \\
\hline $3 / 23 / 01 \quad 16: 00$ & 23.2 & -- & 8.08 & 7.06 & -- & 30.4 \\
\hline $3 / 23 / 01 \quad 17: 00$ & 24.8 & -- & 8.12 & 6.53 & -- & 24.3 \\
\hline $3 / 23 / 01$ 18:00 & 23.2 & -- & 8.16 & 6.02 & -- & 21.4 \\
\hline $3 / 23 / 01$ 19:00 & 22.2 & -- & 8.12 & 5.31 & -- & -- \\
\hline $3 / 23 / 0120: 00$ & 20.8 & -- & 8.05 & 4.59 & -- & -- \\
\hline \multicolumn{7}{|c|}{ April 26-27, 2001} \\
\hline $4 / 26 / 0110: 15$ & -- & - & 8.00 & -- & -- & 41.3 \\
\hline $4 / 26 / 0110: 30$ & 48.7 & 226 & 8.00 & 7.62 & 10.58 & -- \\
\hline 4/26/01 11:00 & 48.7 & 227 & 8.04 & 8.46 & 10.47 & 37.5 \\
\hline $4 / 26 / 0112: 00$ & 47.2 & 229 & 8.11 & 10.17 & 10.12 & 31.1 \\
\hline 4/26/01 13:00 & 46.4 & 231 & 8.13 & 11.79 & 9.76 & 25.7 \\
\hline $4 / 26 / 0114: 00$ & 46.4 & 235 & 8.10 & 12.21 & 9.48 & 19.2 \\
\hline $4 / 26 / 0115: 00$ & 44.9 & 235 & 8.21 & 12.58 & 9.41 & 17.2 \\
\hline $4 / 26 / 0116: 00$ & 44.2 & 236 & 8.23 & 13.19 & 9.16 & 18.3 \\
\hline $4 / 26 / 0117: 00$ & 43.4 & 236 & 8.15 & 13.35 & 8.92 & 16.3 \\
\hline 4/26/01 18:00 & 42.7 & 237 & 8.12 & 13.30 & 8.83 & 15.8 \\
\hline $4 / 26 / 0119: 00$ & 42.7 & 238 & 8.00 & 12.88 & 8.68 & 15.8 \\
\hline 4/26/01 20:00 & 44.2 & 237 & 7.89 & 12.36 & 8.64 & 18.0 \\
\hline $4 / 26 / 0121: 00$ & 46.4 & 234 & 7.84 & 11.74 & 8.71 & 22.3 \\
\hline $4 / 26 / 0122: 00$ & 49.5 & 233 & 7.80 & 11.02 & 8.96 & 30.5 \\
\hline $4 / 26 / 0123: 00$ & 51.9 & 232 & 7.76 & 10.27 & 9.14 & 33.3 \\
\hline $4 / 27 / 01 \quad 0: 00$ & 55.2 & 226 & 7.71 & 9.38 & 9.38 & 35.2 \\
\hline $4 / 27 / 01 \quad 1: 00$ & 57.8 & 219 & 7.71 & 8.48 & 9.62 & 36.8 \\
\hline
\end{tabular}


Table 4. Water-quality data for diel investigations in Prickly Pear Creek at site PP-5, Montana, March-April 2001(Continued).

\begin{tabular}{|c|c|c|c|c|c|c|}
\hline Date and time & $\begin{array}{c}\text { Streamflow, } \\
\text { instantaneous } \\
\left(\mathrm{ft}^{3} / \mathbf{s}\right)\end{array}$ & $\begin{array}{c}\text { Specific } \\
\text { conductance, } \\
\text { field } \\
(\mu \mathrm{S} / \mathrm{cm}) \\
\end{array}$ & $\begin{array}{l}\text { pH, } \\
\text { field } \\
\text { (su) }\end{array}$ & $\begin{array}{c}\text { Temp- } \\
\text { erature, } \\
\text { water } \\
\left({ }^{0} \mathrm{C}\right) \\
\end{array}$ & $\begin{array}{c}\text { Dissolved } \\
\text { oxygen, } \\
\text { field } \\
(\mathrm{mg} / \mathrm{L})\end{array}$ & $\begin{array}{c}\text { Zinc, } \\
\text { dissolved } \\
(\mu \mathrm{g} / \mathrm{L})\end{array}$ \\
\hline \multicolumn{7}{|c|}{ April 26-27, 2001 (Continued) } \\
\hline $4 / 27 / 01 \quad 2: 00$ & 58.6 & 214 & 7.65 & 7.63 & 9.85 & 39.9 \\
\hline 4/27/01 $3: 00$ & 60.3 & 205 & 7.72 & 6.93 & 10.00 & 40.3 \\
\hline $4 / 27 / 01 \quad 4: 00$ & 61.2 & 204 & 7.72 & 6.43 & 10.14 & 41.4 \\
\hline $4 / 27 / 01 \quad 5: 00$ & 61.2 & 203 & 7.71 & 6.03 & 10.28 & 42.0 \\
\hline 4/27/01 $\quad 6: 00$ & 61.2 & 206 & 7.70 & 5.75 & 10.42 & 43.6 \\
\hline 4/27/01 7:00 & 60.3 & 203 & 7.71 & 5.61 & 10.54 & 42.7 \\
\hline 4/27/01 $8: 00$ & 59.5 & 203 & 7.74 & 5.78 & 10.61 & 40.9 \\
\hline 4/27/01 9:00 & 58.6 & 203 & 7.88 & 6.48 & 10.52 & 41.7 \\
\hline $4 / 27 / 01 \quad 10: 00$ & 57.8 & 205 & 7.97 & 7.61 & 10.41 & 37.7 \\
\hline 4/27/01 11:00 & 57.8 & 206 & 8.04 & 9.01 & 10.21 & 31.4 \\
\hline 4/27/01 12:00 & 56.0 & 206 & 8.15 & 10.81 & 9.67 & 27.6 \\
\hline 4/27/01 13:00 & 55.2 & 208 & 8.16 & 12.12 & 9.18 & 21.0 \\
\hline 4/27/01 14:00 & 54.4 & 208 & 8.12 & 12.39 & 9.00 & 22.3 \\
\hline 4/27/01 15:00 & 53.5 & 208 & 8.02 & 12.09 & 8.85 & 19.0 \\
\hline 4/27/01 16:00 & 52.7 & 210 & 8.05 & 12.51 & 9.01 & 20.4 \\
\hline 4/27/01 17:00 & 51.9 & 208 & 8.10 & 12.54 & 8.99 & 18.6 \\
\hline 4/27/01 18:00 & 51.9 & 209 & 8.07 & 12.35 & 8.91 & 22.7 \\
\hline 4/27/01 19:00 & 53.5 & 209 & 7.96 & 11.94 & 8.80 & 21.7 \\
\hline 4/27/01 20:00 & 56.0 & 208 & 7.86 & 11.36 & 8.78 & 23.6 \\
\hline 4/27/01 21:00 & 58.6 & 206 & 7.81 & 10.67 & 8.77 & 27.6 \\
\hline 4/27/01 22:00 & 62.9 & 203 & 7.75 & 9.90 & 9.06 & 32.4 \\
\hline
\end{tabular}


Table 5. Water-quality data for diel investigations in Prickly Pear Creek at site PP-4b, Montana, July 25-27, 2001.

[Samples analyzed by the U.S. Geological Survey laboratory, Boulder, Colo. Analytical data are reported to a maximum of three significant figures to provide sufficient resolution for detecting smal differences between samples closely spaced in time or distance. Similarly, field measurements from electronicrecorders have not been rounded to illustrate the pattern of relative change between measurements. Abbreviations: B, blank sample; $\mathrm{ft}^{3} / \mathrm{s}$, cubic feet per second; ${ }^{\circ} \mathrm{C}$, degrees Celsius, $\mu \mathrm{g} / \mathrm{L}$, micrograms per liter; $\mu \mathrm{S} / \mathrm{cm}$, microsiemens per centimeter at $25^{\circ} \mathrm{C}$; $\mathrm{mg} / \mathrm{L}$, milligrams per liter; su, standard units; QA, qualityassurance; R, replicate sample. Symbol: <, less than; --, no data].

\begin{tabular}{|c|c|c|c|c|c|c|c|c|c|c|c|c|c|c|c|c|}
\hline Date and time & $\begin{array}{c}\text { QA } \\
\text { sample } \\
\text { type }\end{array}$ & $\begin{array}{l}\text { Stream- } \\
\text { flow, } \\
\text { instan- } \\
\text { taneous } \\
\left(\mathrm{ft}^{3} / \mathbf{s}\right)\end{array}$ & $\begin{array}{c}\text { Specific } \\
\text { conduct- } \\
\text { ance, field } \\
(\mu \mathrm{S} / \mathrm{cm})\end{array}$ & $\begin{array}{l}\mathrm{pH}, \\
\text { field } \\
\text { (su) }\end{array}$ & $\begin{array}{c}\text { Tempera- } \\
\text { ture, } \\
\text { water } \\
\left({ }^{\circ} \mathrm{C}\right)\end{array}$ & $\begin{array}{c}\text { Dis- } \\
\text { solved } \\
\text { oxygen, } \\
\text { field } \\
(\mathbf{m g} / \mathrm{L})\end{array}$ & $\begin{array}{c}\text { Cal- } \\
\text { cium, } \\
\text { dis- } \\
\text { solved } \\
(\mathrm{mg} / \mathrm{L})\end{array}$ & $\begin{array}{l}\text { Magne- } \\
\text { sium, } \\
\text { dissolved } \\
(\mathrm{mg} / \mathrm{L})\end{array}$ & $\begin{array}{c}\text { Sodium, } \\
\text { dis- } \\
\text { solved } \\
(\mathrm{mg} / \mathrm{L})\end{array}$ & $\begin{array}{c}\text { Potas- } \\
\text { sium, } \\
\text { dis- } \\
\text { solved } \\
(\mathrm{mg} / \mathrm{L})\end{array}$ & $\begin{array}{c}\text { Alka- } \\
\text { linity, } \\
\text { lab } \\
(\mathbf{m g} / \mathrm{L} \text { as } \\
\mathrm{CaCO}_{3} \text { ) }\end{array}$ & $\begin{array}{c}\text { Sulfate, } \\
\text { dis- } \\
\text { solved } \\
(\mathrm{mg} \text { as } \\
\left.\mathrm{SO}_{4}\right)\end{array}$ & $\begin{array}{c}\text { Chloride, } \\
\text { dissolved } \\
\text { (mg/L) }\end{array}$ & $\begin{array}{c}\text { Fluo- } \\
\text { ride, } \\
\text { dis- } \\
\text { solved } \\
(\mathrm{mg} / \mathrm{L})\end{array}$ & $\begin{array}{c}\text { Silica, } \\
\text { dis- } \\
\text { solved } \\
(\mathrm{mg} / \mathrm{L} \\
\text { as } \\
\left.\mathrm{SiO}_{2}\right) \\
\end{array}$ & $\begin{array}{c}\text { Nitrate, } \\
\text { dissolved } \\
(\mathrm{mg} / \mathrm{L} \text { as } \\
\left.\mathrm{NO}_{3}\right)\end{array}$ \\
\hline 7/25/2001 12:00 & & 19.4 & 239 & 8.30 & 15.94 & 9.22 & 26.9 & 5.99 & 14.5 & 2.20 & 85.7 & 42.9 & 2.83 & 0.43 & 21.5 & 0.63 \\
\hline 7/25/2001 13:00 & & 19.0 & 238 & 8.38 & 17.75 & 8.96 & 26.7 & 6.02 & 14.5 & 2.22 & 85.1 & 42.6 & 2.87 & 0.42 & 21.2 & 0.61 \\
\hline 7/25/2001 14:00 & & 18.6 & 238 & 8.45 & 19.39 & 8.62 & 26.8 & 5.88 & 14.3 & 2.22 & 84.7 & 43.0 & 2.93 & 0.45 & 21.2 & 0.46 \\
\hline 7/25/2001 15:00 & & 18.2 & 238 & 8.33 & 20.73 & 8.35 & 26.8 & 5.93 & 14.4 & 2.27 & 84.1 & 41.5 & 2.90 & 0.45 & 21.5 & 0.57 \\
\hline 7/25/2001 16:00 & & 18.2 & 239 & 8.33 & 21.72 & 8.07 & 26.7 & 5.87 & 14.4 & 2.24 & 84.0 & 43.6 & 2.92 & 0.45 & 21.4 & 0.64 \\
\hline 7/25/2001 17:00 & & 17.4 & 240 & 8.31 & 21.61 & 7.83 & 26.9 & 5.91 & 14.5 & 2.30 & 84.5 & 43.7 & 2.85 & 0.43 & 21.3 & 0.63 \\
\hline 7/25/2001 18:00 & & 17.4 & 241 & 8.29 & 21.22 & 7.84 & 27.0 & 5.85 & 14.6 & 2.35 & 83.5 & 43.4 & 2.88 & 0.44 & 21.2 & 1.18 \\
\hline 7/25/2001 19:00 & & 17.4 & 243 & 8.24 & 20.71 & 7.82 & 27.0 & 5.71 & 14.4 & 2.35 & 85.5 & 43.9 & 2.94 & 0.45 & 21.7 & 0.52 \\
\hline $7 / 25 / 200120: 00$ & & 17.4 & 244 & 8.20 & 20.01 & 7.71 & 27.5 & 6.02 & 14.9 & 2.34 & 86.2 & 43.8 & 2.95 & 0.42 & 21.5 & 0.52 \\
\hline $7 / 25 / 200121: 00$ & & 17.4 & 245 & 8.14 & 19.08 & 7.74 & 27.6 & 6.03 & 15.1 & 2.36 & 87.0 & 44.1 & 2.95 & 0.41 & 21.9 & 0.67 \\
\hline 7/25/2001 22:00 & & 17.0 & 246 & 8.13 & 18.12 & 7.85 & 27.2 & 6.08 & 15.1 & 2.38 & 88.1 & 43.4 & 3.00 & 0.44 & 21.5 & 0.80 \\
\hline 7/25/2001 23:00 & & 17.0 & 246 & 8.14 & 17.07 & 8.06 & 27.6 & 6.06 & 15.2 & 2.35 & 89.0 & 44.0 & 3.00 & 0.44 & 21.9 & 0.71 \\
\hline 7/25/2001 23:00 & $\mathrm{R}$ & -- & -- & -- & -- & -- & 27.0 & 6.06 & 14.8 & 2.37 & 88.2 & 42.7 & 2.94 & 0.42 & 21.2 & 0.98 \\
\hline $7 / 26 / 2001 \quad 0: 00$ & & 17.0 & 246 & 8.14 & 16.18 & 8.22 & 27.3 & 6.00 & 15.1 & 2.37 & 89.1 & 44.5 & 2.94 & 0.43 & 22.0 & 0.73 \\
\hline $7 / 26 / 2001 \quad 1: 00$ & & 17.0 & 247 & 8.14 & 15.42 & 8.38 & 27.5 & 6.12 & 15.4 & 2.34 & 89.8 & 44.2 & 3.04 & 0.43 & 21.9 & 0.72 \\
\hline $7 / 26 / 2001 \quad 2: 00$ & & 17.4 & 246 & 8.14 & 14.69 & 8.55 & 27.7 & 6.02 & 15.3 & 2.39 & 90.1 & 44.5 & 3.10 & 0.46 & 21.6 & 0.88 \\
\hline $7 / 26 / 2001 \quad 3: 00$ & & 17.8 & 246 & 8.12 & 14.09 & 8.75 & 27.7 & 6.00 & 15.4 & 2.34 & 89.7 & 44.6 & 2.97 & 0.41 & 21.5 & 0.84 \\
\hline $7 / 26 / 2001 \quad 4: 00$ & & 17.8 & 245 & 8.10 & 13.40 & 8.85 & 27.6 & 6.03 & 15.4 & 2.34 & 89.3 & 44.2 & 3.05 & 0.43 & 21.3 & 0.87 \\
\hline $7 / 26 / 2001 \quad 5: 00$ & & 17.8 & 244 & 8.12 & 12.79 & 9.00 & 26.8 & 5.84 & 15.2 & 2.26 & 89.6 & 43.3 & 3.07 & 0.47 & 20.8 & 0.83 \\
\hline $7 / 26 / 2001 \quad 6: 00$ & & 17.8 & 243 & 8.16 & 12.29 & 9.16 & 27.4 & 5.94 & 15.2 & 2.35 & 89.4 & 44.2 & 2.96 & 0.41 & 21.9 & 0.80 \\
\hline $7 / 26 / 2001 \quad 7: 00$ & & 17.8 & 242 & 8.18 & 11.92 & 9.29 & 27.4 & 5.87 & 15.4 & 2.28 & 89.2 & 44.4 & 2.95 & 0.42 & 21.7 & 0.78 \\
\hline $7 / 26 / 2001 \quad 8: 00$ & & 18.2 & 242 & 8.21 & 11.91 & 9.54 & 27.4 & 5.82 & 15.2 & 2.29 & 88.9 & 44.2 & 2.93 & 0.44 & 21.6 & 0.80 \\
\hline
\end{tabular}


Table 5. Water-quality data for diel investigations in Prickly Pear Creek at site PP-4b, Montana, July 25-27, 2001 (Continued).

[Samples analyzed by the U.S. Geological Survey laboratory, Boulder, Colo. Analytical data are reported to a maximum of three significant figures to provide sufficient resolution for detecting small differences between samples closely spaced in time or distance. Similarly, field measurements from electronic recorders have not been rounded to illustrate the pattern of relative change between measurements. Abbreviations: B, blank sample; $\mathrm{ft}^{3} / \mathrm{s}$, cubic feet per second; ${ }^{\circ} \mathrm{C}$, degrees Celsius, $\mu \mathrm{g} / \mathrm{L}$, micrograms per liter; $\mu \mathrm{S} / \mathrm{cm}$, microsiemens per centimeter at $25^{\circ} \mathrm{C} ; \mathrm{mg} / \mathrm{L}$, milligrams per liter; su, standard units; QA, quality assurance; R, replicate sample. Symbol: <, less than; --, no data].

\begin{tabular}{|c|c|c|c|c|c|c|c|c|c|c|c|c|c|c|c|c|}
\hline Date and time & $\begin{array}{c}\text { QA } \\
\text { sample } \\
\text { type }\end{array}$ & $\begin{array}{c}\text { Alum- } \\
\text { inum, } \\
\text { total } \\
\text { recov- } \\
\text { erable } \\
(\mu \mathrm{g} / \mathrm{L})\end{array}$ & $\begin{array}{c}\text { Alum- } \\
\text { inum, } \\
\text { dis- } \\
\text { solved } \\
(\mu \mathrm{g} / \mathrm{L})\end{array}$ & $\begin{array}{c}\text { Arsenic, } \\
\text { total } \\
\text { recov- } \\
\text { erable } \\
(\mu \mathrm{g} / \mathrm{L})\end{array}$ & $\begin{array}{c}\text { Arsenic } \\
, \mathbf{A s}^{+3}+ \\
\text { As }^{+5} \\
\text { dis- } \\
\text { solved } \\
(\mu \mathrm{g} / \mathrm{L}) \\
\end{array}$ & $\begin{array}{c}\text { Arsenic, } \\
\text { As }^{+3}, \\
\text { dis- } \\
\text { solved } \\
(\mu \mathrm{g} / \mathrm{L}) \\
\end{array}$ & $\begin{array}{c}\text { Barium, } \\
\text { dis- } \\
\text { solved } \\
(\mu \mathrm{g} / \mathrm{L})\end{array}$ & $\begin{array}{c}\text { Beryl- } \\
\text { lium, } \\
\text { dis- } \\
\text { solved } \\
(\mu \mathrm{g} / \mathrm{L})\end{array}$ & $\begin{array}{c}\text { Boron, } \\
\text { dis- } \\
\text { solved } \\
(\mu \mathrm{g} / \mathrm{L})\end{array}$ & $\begin{array}{c}\text { Cad- } \\
\text { mium, } \\
\text { total } \\
\text { recov- } \\
\text { erable } \\
(\mu \mathrm{g} / \mathrm{L}) \\
\end{array}$ & $\begin{array}{c}\text { Cad- } \\
\text { mium, } \\
\text { dis- } \\
\text { solved } \\
(\mu \mathrm{g} / \mathrm{L})\end{array}$ & $\begin{array}{c}\text { Chro- } \\
\text { mium, } \\
\text { dis- } \\
\text { solved } \\
(\mu \mathrm{g} / \mathrm{L})\end{array}$ & $\begin{array}{c}\text { Cobalt, } \\
\text { dis- } \\
\text { solved } \\
(\mu \mathrm{g} / \mathrm{L})\end{array}$ & $\begin{array}{c}\text { Copper, } \\
\text { total } \\
\text { recov- } \\
\text { erable } \\
(\mu \mathrm{g} / \mathrm{L})\end{array}$ & $\begin{array}{c}\text { Copper, } \\
\text { dis- } \\
\text { solved } \\
(\mu \mathrm{g} / \mathrm{L})\end{array}$ & $\begin{array}{c}\text { Iron, } \\
\text { total } \\
\text { recov- } \\
\text { erable } \\
(\mu \mathrm{g} / \mathrm{L})\end{array}$ \\
\hline 7/25/2001 12:00 & & $<80$ & $<80$ & $<50$ & 3.03 & 0.61 & 25.8 & $<0.1$ & 19.4 & $<1$ & $<1$ & $<1$ & $<1$ & 2.22 & 2.12 & 151 \\
\hline 7/25/2001 13:00 & & $<80$ & $<80$ & $<50$ & 4.58 & $<0.5$ & 25.1 & $<0.1$ & 19.4 & $<1$ & $<1$ & $<1$ & $<1$ & 2.46 & 1.85 & 147 \\
\hline 7/25/2001 14:00 & & $<80$ & $<80$ & $<50$ & 4.97 & $<0.5$ & 25.2 & $<0.1$ & 19.1 & $<1$ & $<1$ & $<1$ & $<1$ & 2.59 & 2.00 & 149 \\
\hline 7/25/2001 15:00 & & $<80$ & $<80$ & $<50$ & 5.21 & $<0.5$ & 24.8 & $<0.1$ & 19.4 & $<1$ & $<1$ & $<1$ & $<1$ & 2.62 & 2.44 & 149 \\
\hline 7/25/2001 16:00 & & $<80$ & $<80$ & $<50$ & 5.40 & $<0.5$ & 24.4 & $<0.1$ & 19.4 & $<1$ & $<1$ & $<1$ & $<1$ & 2.61 & 2.28 & 146 \\
\hline 7/25/2001 17:00 & & $<80$ & $<80$ & $<50$ & 5.60 & 0.56 & 24.8 & $<0.1$ & 19.6 & $<1$ & $<1$ & $<1$ & $<1$ & 2.59 & 2.17 & 140 \\
\hline 7/25/2001 18:00 & & $<80$ & $<80$ & $<50$ & 5.69 & 0.77 & 24.8 & $<0.1$ & 20.4 & $<1$ & $<1$ & $<1$ & $<1$ & 2.67 & 2.41 & 145 \\
\hline 7/25/2001 19:00 & & $<80$ & $<80$ & $<50$ & 5.80 & 0.60 & 25.3 & $<0.1$ & 20.6 & $<1$ & $<1$ & $<1$ & $<1$ & 2.72 & 1.79 & 152 \\
\hline $7 / 25 / 200120: 00$ & & $<80$ & $<80$ & $<50$ & 5.06 & 0.54 & 25.7 & $<0.1$ & 20.7 & $<1$ & $<1$ & $<1$ & $<1$ & 2.84 & 2.21 & 145 \\
\hline 7/25/2001 21:00 & & $<80$ & $<80$ & $<50$ & 5.42 & 0.97 & 26.2 & $<0.1$ & 21.5 & $<1$ & $<1$ & $<1$ & $<1$ & 3.13 & 1.78 & 160 \\
\hline 7/25/2001 23:00 & & $<80$ & $<80$ & $<50$ & 5.03 & $<0.5$ & 27.6 & $<0.1$ & 20.5 & $<1$ & $<1$ & $<1$ & $<1$ & 2.68 & 2.55 & 188 \\
\hline 7/25/2001 23:00 & $\mathrm{R}$ & $<80$ & $<80$ & $<50$ & 4.95 & $<0.5$ & 27.9 & $<0.1$ & 20.2 & $<1$ & $<1$ & $<1$ & $<1$ & 2.69 & 2.28 & 256 \\
\hline $7 / 26 / 2001 \quad 0: 00$ & & $<80$ & $<80$ & $<50$ & 4.84 & $<0.5$ & 27.7 & $<0.1$ & 20.0 & $<1$ & $<1$ & $<1$ & $<1$ & 2.62 & 1.77 & 183 \\
\hline $7 / 26 / 2001 \quad 1: 00$ & & $<80$ & $<80$ & $<50$ & 4.66 & $<0.5$ & 27.9 & $<0.1$ & 20.0 & $<1$ & $<1$ & $<1$ & $<1$ & 2.63 & 1.87 & 165 \\
\hline $7 / 26 / 2001 \quad 2: 00$ & & $<80$ & $<80$ & $<50$ & 4.51 & $<0.5$ & 27.5 & $<0.1$ & 20.5 & $<1$ & $<1$ & $<1$ & $<1$ & 2.93 & 1.74 & 159 \\
\hline $7 / 26 / 2001 \quad 3: 00$ & & $<80$ & $<80$ & $<50$ & 4.47 & $<0.5$ & 27.0 & $<0.1$ & 19.6 & $<1$ & $<1$ & $<1$ & $<1$ & 2.89 & 1.68 & 159 \\
\hline $7 / 26 / 2001 \quad 4: 00$ & & $<80$ & $<80$ & $<50$ & 4.55 & $<0.5$ & 27.5 & $<0.1$ & 19.7 & $<1$ & $<1$ & $<1$ & $<1$ & 2.60 & 1.90 & 164 \\
\hline $7 / 26 / 2001 \quad 5: 00$ & & $<80$ & $<80$ & $<50$ & 4.36 & $<0.5$ & 27.0 & $<0.1$ & 20.8 & $<1$ & $<1$ & $<1$ & $<1$ & 2.53 & 1.80 & 145 \\
\hline $7 / 26 / 2001 \quad 6: 00$ & & $<80$ & $<80$ & $<50$ & 4.00 & $<0.5$ & 27.7 & $<0.1$ & 19.7 & $<1$ & $<1$ & $<1$ & $<1$ & 2.80 & 1.98 & 157 \\
\hline $7 / 26 / 2001 \quad 7: 00$ & & $<80$ & $<80$ & $<50$ & 4.45 & 0.53 & 27.1 & $<0.1$ & 18.8 & $<1$ & $<1$ & $<1$ & $<1$ & 2.64 & 3.60 & 168 \\
\hline $7 / 26 / 2001 \quad 8: 00$ & & $<80$ & $<80$ & $<50$ & 4.45 & $<0.5$ & 26.7 & $<0.1$ & 19.6 & $<1$ & $<1$ & $<1$ & $<1$ & 2.30 & 1.85 & 157 \\
\hline
\end{tabular}


Table 5. Water-quality data for diel investigations in Prickly Pear Creek at site PP-4b, Montana, July 25-27, 2001 (Continued).

[Samples analyzed by the U.S. Geological Survey laboratory, Boulder, Colo. Analytical data are reported to a maximum of three significant figures to provide sufficient resolution for detecting small differences between samples closely spaced in time or distance. Similarly, field measurements from electronic recorders have not been rounded to illustrate the pattern of relative change between

measurements. Abbreviations: B, blank sample; $\mathrm{ft}^{3} / \mathrm{s}$, cubic feet per second; ${ }^{\circ} \mathrm{C}$, degrees Celsius, $\mu \mathrm{g} / \mathrm{L}$, micrograms per liter; $\mu \mathrm{S} / \mathrm{cm}$, microsiemens per centimeter at $25^{\circ} \mathrm{C} ; \mathrm{mg} / \mathrm{L}$, milligrams per liter; su, standard units; QA, quality assurance; R, replicate sample. Symbol: <, less than; --, no data].

\begin{tabular}{|c|c|c|c|c|c|c|c|c|c|c|c|c|c|c|c|c|c|c|}
\hline Date and time & $\begin{array}{c}\text { QA } \\
\text { sample } \\
\text { type }\end{array}$ & $\begin{array}{c}\text { Iron, } \\
\mathrm{Fe}^{+2}+ \\
\mathrm{Fe}^{+3}, \\
\text { dis- } \\
\text { solved } \\
(\mu \mathrm{g} / \mathrm{L}) \\
\end{array}$ & $\begin{array}{c}\text { Iron, } \\
\text { Fe }^{+2}, \\
\text { dis- } \\
\text { solved } \\
(\mu \mathrm{g} / \mathrm{L})\end{array}$ & $\begin{array}{c}\text { Lead, } \\
\text { total } \\
\text { recov- } \\
\text { erable } \\
(\mu \mathrm{g} / \mathrm{L})\end{array}$ & $\begin{array}{c}\text { Lead, } \\
\text { dis- } \\
\text { solved } \\
(\mu \mathrm{g} / \mathrm{L})\end{array}$ & $\begin{array}{c}\text { Lith- } \\
\text { ium, } \\
\text { dis- } \\
\text { solved } \\
(\mu \mathrm{g} / \mathrm{L})\end{array}$ & $\begin{array}{c}\text { Manga- } \\
\text { nese, } \\
\text { total } \\
\text { recov- } \\
\text { erable } \\
(\mu \mathrm{g} / \mathrm{L})\end{array}$ & $\begin{array}{l}\text { Manga- } \\
\text { nese, } \\
\text { dis- } \\
\text { solved } \\
(\mu \mathrm{g} / \mathrm{L})\end{array}$ & $\begin{array}{c}\text { Nickel, } \\
\text { total } \\
\text { recov- } \\
\text { erable } \\
(\mu \mathrm{g} / \mathrm{L})\end{array}$ & $\begin{array}{l}\text { Nickel, } \\
\text { dis- } \\
\text { solved } \\
(\mu \mathrm{g} / \mathrm{L})\end{array}$ & $\begin{array}{c}\text { Sele- } \\
\text { nium, } \\
\text { total } \\
\text { recov- } \\
\text { erable } \\
(\mu \mathrm{g} / \mathrm{L}) \\
\end{array}$ & $\begin{array}{c}\text { Sele- } \\
\text { nium, } \\
\text { dis- } \\
\text { solved } \\
(\mu \mathrm{g} / \mathrm{L})\end{array}$ & 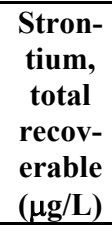 & $\begin{array}{c}\text { Stron- } \\
\text { tium, } \\
\text { dis- } \\
\text { solved } \\
(\mu \mathrm{g} / \mathrm{L})\end{array}$ & $\begin{array}{c}\text { Vana- } \\
\text { dium, } \\
\text { total } \\
\text { recov- } \\
\text { erable } \\
(\mu \mathrm{g} / \mathrm{L}) \\
\end{array}$ & $\begin{array}{c}\text { Vana- } \\
\text { dium, } \\
\text { dis- } \\
\text { solved } \\
(\mu \mathrm{g} / \mathrm{L})\end{array}$ & $\begin{array}{c}\text { Zinc, } \\
\text { total } \\
\text { recov- } \\
\text { erable } \\
(\mu \mathrm{g} / \mathrm{L})\end{array}$ & $\begin{array}{c}\text { Zinc, } \\
\text { dis- } \\
\text { solved } \\
(\mu \mathrm{g} / \mathrm{L})\end{array}$ \\
\hline 7/25/2001 12:00 & & 45.6 & 26.5 & $<8$ & $<8$ & 20.1 & 48.6 & 41.5 & $<2$ & $<2$ & $<50$ & $<50$ & 241 & 239 & $<1$ & $<1$ & 72.5 & 62.6 \\
\hline 7/25/2001 13:00 & & 39.9 & 25.6 & $<8$ & $<8$ & 19.8 & 45.9 & 37.2 & $<2$ & $<2$ & $<50$ & $<50$ & 237 & 238 & $<1$ & $<1$ & 61.7 & 49.5 \\
\hline 7/25/2001 14:00 & & 37.8 & 29.7 & $<8$ & $<8$ & 20.4 & 44.8 & 36.2 & $<2$ & $<2$ & $<50$ & $<50$ & 239 & 240 & $<1$ & $<1$ & 54.0 & 43.0 \\
\hline 7/25/2001 15:00 & & 36.8 & 24.6 & $<8$ & $<8$ & 20.2 & 42.9 & 33.9 & $<2$ & $<2$ & $<50$ & $<50$ & 243 & 239 & 1.0 & $<1$ & 46.5 & 34.8 \\
\hline $7 / 25 / 2001$ 16:00 & & 33.8 & 24.1 & $<8$ & $<8$ & 20.2 & 42.1 & 32.5 & $<2$ & $<2$ & $<50$ & $<50$ & 239 & 237 & 1.1 & $<1$ & 42.4 & 29.9 \\
\hline 7/25/2001 17:00 & & 34.8 & 29.7 & $<8$ & $<8$ & 20.8 & 40.9 & 33.0 & $<2$ & $<2$ & $<50$ & $<50$ & 237 & 241 & 1.0 & $<1$ & 40.0 & 28.0 \\
\hline $7 / 25 / 2001$ 18:00 & & 32.3 & 28.1 & $<8$ & $<8$ & 21.4 & 42.9 & 34.6 & $<2$ & $<2$ & $<50$ & $<50$ & 242 & 243 & 1.1 & $<1$ & 41.8 & 30.6 \\
\hline 7/25/2001 19:00 & & 35.9 & 29.9 & $<8$ & $<8$ & 21.7 & 45.6 & 36.9 & $<2$ & $<2$ & $<50$ & $<50$ & 243 & 243 & $<1$ & $<1$ & 44.6 & 31.0 \\
\hline $7 / 25 / 200120: 00$ & & 35.7 & 34.9 & $<8$ & $<8$ & 21.6 & 48.5 & 39.5 & $<2$ & $<2$ & $<50$ & $<50$ & 246 & 245 & 1.2 & $<1$ & 48.1 & 35.5 \\
\hline 7/25/2001 21:00 & & 40.8 & 34.2 & $<8$ & $<8$ & 21.8 & 52.2 & 43.7 & $<2$ & $<2$ & $<50$ & $<50$ & 248 & 247 & 1.0 & $<1$ & 56.8 & 43.8 \\
\hline $7 / 25 / 200122: 00$ & & 44.2 & 36.9 & $<8$ & $<8$ & 22.2 & 70.2 & 48.4 & $<2$ & $<2$ & $<50$ & $<50$ & 250 & 248 & 1.0 & $<1$ & 72.2 & 51.9 \\
\hline 7/25/2001 23:00 & & 42.7 & 39.7 & $<8$ & $<8$ & 21.7 & 63.4 & 51.7 & 2.1 & $<2$ & $<50$ & $<50$ & 248 & 252 & $<1$ & $<1$ & 76.5 & 62.2 \\
\hline $7 / 25 / 200123: 00$ & $\mathrm{R}$ & 43.3 & 32.5 & $<8$ & $<8$ & 21.0 & 73.5 & 51.0 & $<2$ & $<2$ & $<50$ & $<50$ & 255 & 248 & 1.2 & $<1$ & 85.7 & 61.8 \\
\hline $7 / 26 / 2001 \quad 0: 00$ & & 43.0 & 34.6 & $<8$ & $<8$ & 21.2 & 65.6 & 53.6 & $<2$ & $<2$ & $<50$ & $<50$ & 250 & 249 & $<1$ & $<1$ & 85.3 & 71.4 \\
\hline $7 / 26 / 2001 \quad 1: 00$ & & 44.3 & 32.1 & $<8$ & $<8$ & 22.5 & 65.1 & 55.3 & $<2$ & $<2$ & $<50$ & $<50$ & 250 & 250 & $<1$ & $<1$ & 92.4 & 80.1 \\
\hline $7 / 26 / 2001 \quad 2: 00$ & & 42.8 & 30.4 & $<8$ & $<8$ & 22.9 & 64.7 & 55.5 & $<2$ & $<2$ & $<50$ & $<50$ & 248 & 252 & $<1$ & $<1$ & 98.3 & 90.0 \\
\hline $7 / 26 / 2001 \quad 3: 00$ & & 41.5 & 29.0 & $<8$ & $<8$ & 22.5 & 66.4 & 55.8 & $<2$ & $<2$ & $<50$ & $<50$ & 247 & 249 & $<1$ & $<1$ & 106 & 94.7 \\
\hline $7 / 26 / 2001 \quad 4: 00$ & & 42.7 & 31.1 & $<8$ & $<8$ & 23.0 & 67.7 & 56.3 & $<2$ & $<2$ & $<50$ & $<50$ & 249 & 249 & $<1$ & $<1$ & 112 & 101 \\
\hline $7 / 26 / 2001 \quad 5: 00$ & & 43.1 & 18.7 & $<8$ & $<8$ & 21.5 & 64.8 & 54.7 & $<2$ & $<2$ & $<50$ & $<50$ & 246 & 249 & $<1$ & $<1$ & 112 & 100 \\
\hline $7 / 26 / 2001 \quad 6: 00$ & & 46.9 & 11.1 & $<8$ & $<8$ & 23.1 & 67.3 & 56.5 & $<2$ & $<2$ & $<50$ & $<50$ & 246 & 250 & $<1$ & $<1$ & 113 & 107 \\
\hline $7 / 26 / 2001 \quad 7: 00$ & & 44.9 & 23.9 & $<8$ & $<8$ & 22.5 & 66.1 & 56.5 & $<2$ & $<2$ & $<50$ & $<50$ & 247 & 248 & $<1$ & $<1$ & 116 & 107 \\
\hline $7 / 26 / 2001 \quad 8: 00$ & & 48.1 & 32.2 & $<8$ & $<8$ & 22.6 & 64.3 & 54.8 & $<2$ & $<2$ & $<50$ & $<50$ & 251 & 248 & $<1$ & $<1$ & 115 & 106 \\
\hline
\end{tabular}


Table 5. Water-quality data for diel investigations in Prickly Pear Creek at site PP-4b, Montana, July 25-27, 2001(Continued).

\begin{tabular}{|c|c|c|c|c|c|c|c|c|c|c|c|c|c|c|c|c|}
\hline Date and time & $\begin{array}{c}\text { QA } \\
\text { sample } \\
\text { type }\end{array}$ & $\begin{array}{c}\text { Stream } \\
\text {-flow, } \\
\text { instan- } \\
\text { taneous } \\
\left(\mathbf{f t}^{3} / \mathbf{s}\right) \\
\end{array}$ & $\begin{array}{c}\text { Specific } \\
\text { conduct- } \\
\text { ance, } \\
\text { field } \\
(\mu \mathrm{S} / \mathrm{cm})\end{array}$ & $\begin{array}{l}\text { pH, } \\
\text { field } \\
\text { (su) }\end{array}$ & $\begin{array}{c}\text { Tempera- } \\
\text { ture, } \\
\text { water } \\
\left({ }^{\circ} \mathrm{C}\right)\end{array}$ & $\begin{array}{c}\text { Dis- } \\
\text { solved } \\
\text { oxygen } \\
\text {, field } \\
(\mathrm{mg} / \mathrm{L}) \\
\end{array}$ & $\begin{array}{c}\text { Cal- } \\
\text { cium, } \\
\text { dis- } \\
\text { solved } \\
(\mathrm{mg} / \mathrm{L})\end{array}$ & $\begin{array}{l}\text { Magne- } \\
\text { sium, } \\
\text { dissolved } \\
(\mathrm{mg} / \mathrm{L})\end{array}$ & $\begin{array}{c}\text { Sodium } \\
\text {, dis- } \\
\text { solved } \\
(\mathrm{mg} / \mathrm{L})\end{array}$ & $\begin{array}{c}\text { Potas- } \\
\text { sium, } \\
\text { dis- } \\
\text { solved } \\
(\mathbf{m g} / \mathbf{L}) \\
\end{array}$ & $\begin{array}{c}\text { Alka- } \\
\text { linity, } \\
\text { lab } \\
(\mathrm{mg} / \mathrm{L} \text { as } \\
\left.\mathrm{CaCO}_{3}\right)\end{array}$ & $\begin{array}{c}\text { Sulfate, } \\
\text { dis- } \\
\text { solved } \\
(\mathrm{mg} \text { as } \\
\left.\mathrm{SO}_{4}\right) \\
\end{array}$ & $\begin{array}{c}\text { Chloride, } \\
\text { dissolved } \\
(\mathrm{mg} / \mathrm{L})\end{array}$ & $\begin{array}{c}\text { Fluo- } \\
\text { ride, } \\
\text { dis- } \\
\text { solved } \\
\text { (mg/L) } \\
\end{array}$ & $\begin{array}{c}\text { Silica, } \\
\text { dis- } \\
\text { solved } \\
(\mathrm{mg} / \mathrm{L} \\
\left.\text { as } \mathrm{SiO}_{2}\right) \\
\end{array}$ & $\begin{array}{c}\text { Nitrate, } \\
\text { dissolved } \\
(\mathrm{mg} / \mathrm{L} \text { as } \\
\left.\mathrm{NO}_{3}\right)\end{array}$ \\
\hline $7 / 26 / 2001 \quad 8: 30$ & B & -- & -- & -- & -- & -- & $<0.05$ & $<0.06$ & $<0.04$ & $<0.002$ & $<0.5$ & $<0.8$ & 0.60 & 0.10 & $<0.01$ & 0.06 \\
\hline 7/26/2001 9:00 & & 18.2 & 242 & 8.25 & 12.34 & 9.64 & 27.3 & 6.03 & 15.3 & 2.30 & 88.6 & 43.6 & 2.94 & 0.41 & 21.6 & 0.66 \\
\hline 7/26/2001 10:00 & & 18.2 & 242 & 8.27 & 13.11 & 9.63 & 27.4 & 6.01 & 15.0 & 2.22 & 88.3 & 44.4 & 2.89 & 0.40 & 21.5 & 0.75 \\
\hline 7/26/2001 11:00 & & 17.8 & 242 & 8.31 & 14.34 & 9.41 & 26.6 & 6.07 & 15.2 & 2.26 & 87.5 & 43.8 & 2.89 & 0.42 & 21.1 & 0.74 \\
\hline 7/26/2001 12:00 & & 17.8 & 243 & 8.33 & 15.91 & 9.21 & 26.7 & 6.00 & 15.1 & 2.31 & 87.2 & 42.3 & 2.90 & 0.41 & 21.1 & 0.62 \\
\hline 7/26/2001 13:00 & & 17.4 & 242 & 8.36 & 17.47 & 8.91 & 26.5 & 5.98 & 14.9 & 2.24 & 86.7 & 42.6 & 2.88 & 0.42 & 20.9 & 0.54 \\
\hline 7/26/2001 14:00 & & 17.4 & 243 & 8.38 & 19.23 & 8.49 & 26.5 & 5.95 & 15.0 & 2.28 & 86.1 & 42.6 & 2.98 & 0.43 & 21.2 & 0.49 \\
\hline 7/26/2001 15:00 & & 17.4 & 243 & 8.39 & 20.67 & 8.13 & 26.6 & 5.99 & 15.1 & 2.31 & 86.0 & 43.0 & 2.90 & 0.43 & 21.2 & 0.45 \\
\hline 7/26/2001 15:00 & $\mathrm{R}$ & -- & -- & -- & -- & -- & 26.3 & 5.94 & 14.8 & 2.30 & 86.3 & 43.2 & 2.92 & 0.45 & 21.3 & 0.07 \\
\hline 7/26/2001 16:00 & & -- & 244 & 8.39 & 21.00 & 7.87 & 26.5 & 5.84 & 15.0 & 2.34 & 85.9 & 42.9 & 2.89 & 0.42 & 21.0 & 0.30 \\
\hline $7 / 26 / 200116: 30$ & & 17.4 & 244 & 8.39 & 21.25 & 7.76 & 26.4 & 5.88 & 14.9 & 2.34 & 86.1 & 43.5 & 2.93 & 0.44 & 21.0 & 0.30 \\
\hline 7/26/2001 17:00 & & -- & 245 & 8.37 & 21.47 & 7.73 & 26.8 & 5.98 & 15.1 & 2.32 & 85.7 & 43.3 & 2.90 & 0.42 & 21.2 & 0.34 \\
\hline $7 / 26 / 2001$ 17:30 & & 17.0 & 245 & 8.36 & 21.19 & 7.57 & 26.6 & 5.94 & 14.9 & 2.33 & 86.3 & 43.6 & 2.95 & 0.42 & 21.3 & 0.36 \\
\hline 7/26/2001 18:00 & & -- & 246 & 8.35 & 21.07 & 7.55 & 26.9 & 5.98 & 15.2 & 2.33 & 86.4 & 43.4 & 2.90 & 0.44 & 21.3 & 0.28 \\
\hline 7/26/2001 18:30 & & 17.0 & 246 & 8.31 & 20.94 & 7.61 & 26.9 & 5.91 & 15.0 & 2.33 & 86.5 & 44.3 & 2.97 & 0.43 & 21.0 & 0.46 \\
\hline 7/26/2001 19:00 & & -- & 247 & 8.30 & 20.81 & 7.53 & 27.3 & 5.99 & 15.2 & 2.37 & 86.8 & 43.7 & 2.98 & 0.45 & 21.3 & 0.54 \\
\hline 7/26/2001 19:30 & & 16.2 & 248 & 8.26 & 20.61 & 7.50 & 27.2 & 5.87 & 15.3 & 2.39 & 87.1 & 43.7 & 2.96 & 0.42 & 21.4 & 0.41 \\
\hline 7/26/2001 20:00 & & 16.2 & 248 & 8.20 & 20.37 & 7.49 & 26.9 & 6.00 & 15.3 & 2.37 & 87.9 & 44.4 & 3.08 & 0.44 & 21.1 & 0.41 \\
\hline 7/26/2001 21:00 & & 16.2 & 249 & 8.17 & 19.55 & 7.40 & 27.5 & 5.98 & 15.4 & 2.37 & 88.6 & 43.4 & 3.10 & 0.45 & 21.3 & 0.57 \\
\hline $7 / 26 / 200122: 00$ & & 16.2 & 250 & 8.14 & 18.62 & 7.51 & 27.3 & 5.98 & 15.5 & 2.36 & 89.6 & 43.0 & 3.07 & 0.45 & 21.2 & 0.56 \\
\hline 7/26/2001 23:00 & & 16.2 & 251 & 8.14 & 17.64 & 7.64 & 27.9 & 6.11 & 15.9 & 2.43 & 90.2 & 42.8 & 3.19 & 0.49 & 21.6 & 0.59 \\
\hline $7 / 27 / 2001 \quad 0: 00$ & & 16.2 & 250 & 8.13 & 16.69 & 7.85 & 27.7 & 6.12 & 15.6 & 2.34 & 90.1 & 42.6 & 3.10 & 0.45 & 21.6 & 0.67 \\
\hline $7 / 27 / 2001 \quad 1: 00$ & & 17.0 & 249 & 8.14 & 15.76 & 8.05 & 27.7 & 6.15 & 15.9 & 2.39 & 90.4 & 42.6 & 3.02 & 0.44 & 21.5 & 0.61 \\
\hline $7 / 27 / 2001 \quad 2: 00$ & & 17.0 & 248 & 8.14 & 14.88 & 8.24 & 27.6 & 6.14 & 15.7 & 2.37 & 90.4 & 41.9 & 2.99 & 0.45 & 21.5 & 0.69 \\
\hline $7 / 27 / 2001 \quad 3: 00$ & & 17.4 & 247 & 8.14 & 14.05 & 8.39 & 27.5 & 6.12 & 15.5 & 2.37 & 89.7 & 41.5 & 3.06 & 0.49 & 21.5 & 0.75 \\
\hline $7 / 27 / 2001 \quad 4: 00$ & & 17.4 & 245 & 8.14 & 13.35 & 8.56 & 27.5 & 6.12 & 15.5 & 2.35 & 89.7 & 41.4 & 2.97 & 0.44 & 21.4 & 0.61 \\
\hline
\end{tabular}


Table 5. Water-quality data for diel investigations in Prickly Pear Creek at site PP-4b, Montana, July 25-27, 2001 (Continued).

\begin{tabular}{|c|c|c|c|c|c|c|c|c|c|c|c|c|c|c|c|c|}
\hline Date and time & $\begin{array}{c}\text { QA } \\
\text { sample } \\
\text { type }\end{array}$ & $\begin{array}{l}\text { Alum- } \\
\text { inum, } \\
\text { total } \\
\text { recov- } \\
\text { erable } \\
(\mu \mathrm{g} / \mathrm{L}) \\
\end{array}$ & $\begin{array}{c}\text { Alum- } \\
\text { inum, } \\
\text { dis- } \\
\text { solved } \\
(\mu \mathrm{g} / \mathrm{L})\end{array}$ & $\begin{array}{c}\text { Arsenic, } \\
\text { total } \\
\text { recov- } \\
\text { erable } \\
(\mu \mathrm{g} / \mathrm{L})\end{array}$ & $\begin{array}{c}\text { Arsenic, } \\
\mathbf{A s}^{+3}+ \\
\mathbf{A s}^{+5} \\
\text { dis- } \\
\text { solved } \\
(\mu \mathrm{g} / \mathrm{L}) \\
\end{array}$ & $\begin{array}{c}\text { Arsenic, } \\
\mathrm{As}^{+3} \\
\text { dis- } \\
\text { solved } \\
(\mu \mathrm{g} / \mathrm{L})\end{array}$ & $\begin{array}{c}\text { Barium, } \\
\text { dis- } \\
\text { solved } \\
(\mu \mathrm{g} / \mathrm{L})\end{array}$ & $\begin{array}{c}\text { Beryl- } \\
\text { lium, } \\
\text { dis- } \\
\text { solved } \\
(\mu \mathrm{g} / \mathrm{L})\end{array}$ & $\begin{array}{c}\text { Boron, } \\
\text { dis- } \\
\text { solved } \\
(\mu \mathrm{g} / \mathrm{L})\end{array}$ & $\begin{array}{c}\text { Cad- } \\
\text { mium, } \\
\text { total } \\
\text { recov- } \\
\text { erable } \\
(\mu \mathrm{g} / \mathrm{L}) \\
\end{array}$ & $\begin{array}{c}\text { Cad- } \\
\text { mium, } \\
\text { dis- } \\
\text { solved } \\
(\mu \mathrm{g} / \mathrm{L})\end{array}$ & $\begin{array}{c}\text { Chro- } \\
\text { mium, } \\
\text { dis- } \\
\text { solved } \\
(\mu \mathrm{g} / \mathrm{L})\end{array}$ & $\begin{array}{c}\text { Cobalt, } \\
\text { dis- } \\
\text { solved } \\
(\mu \mathrm{g} / \mathrm{L})\end{array}$ & $\begin{array}{c}\text { Copper, } \\
\text { total } \\
\text { recov- } \\
\text { erable } \\
(\mu \mathrm{g} / \mathrm{L})\end{array}$ & $\begin{array}{c}\text { Copper, } \\
\text { dis- } \\
\text { solved } \\
(\mu \mathrm{g} / \mathrm{L})\end{array}$ & $\begin{array}{l}\text { Iron, } \\
\text { total } \\
\text { recov- } \\
\text { erable } \\
(\mu \mathrm{g} / \mathrm{L})\end{array}$ \\
\hline $7 / 26 / 2001 \quad 8: 30$ & B & $<80$ & $<80$ & $<50$ & $<0.1$ & $<0.5$ & $<0.5$ & $<0.1$ & $<3$ & $<1$ & $<1$ & $<1$ & $<1$ & $<0.5$ & $<0.5$ & $<9$ \\
\hline $7 / 26 / 2001 \quad 9: 00$ & & $<80$ & $<80$ & $<50$ & 4.48 & $<0.5$ & 26.6 & $<0.1$ & 19.0 & $<1$ & $<1$ & $<1$ & $<1$ & 2.42 & 1.93 & 148 \\
\hline 7/26/2001 10:00 & & $<80$ & $<80$ & $<50$ & 4.32 & $<0.5$ & 26.1 & $<0.1$ & 18.5 & $<1$ & $<1$ & $<1$ & $<1$ & 2.15 & 2.20 & 138 \\
\hline 7/26/2001 11:00 & & $<80$ & $<80$ & $<50$ & 3.50 & $<0.5$ & 25.6 & $<0.1$ & 19.6 & $<1$ & $<1$ & $<1$ & $<1$ & 1.90 & 2.03 & 138 \\
\hline 7/26/2001 12:00 & & $<80$ & $<80$ & $<50$ & 4.67 & $<0.5$ & 25.8 & $<0.1$ & 19.3 & $<1$ & $<1$ & $<1$ & $<1$ & 2.03 & 1.78 & 137 \\
\hline 7/26/2001 13:00 & & $<80$ & $<80$ & $<50$ & 4.78 & $<0.5$ & 25.2 & $<0.1$ & 19.0 & $<1$ & $<1$ & $<1$ & $<1$ & 2.16 & 1.96 & 135 \\
\hline 7/26/2001 14:00 & & $<80$ & $<80$ & $<50$ & 4.96 & $<0.5$ & 25.2 & $<0.1$ & 19.1 & $<1$ & $<1$ & $<1$ & $<1$ & 2.63 & 1.93 & 133 \\
\hline 7/26/2001 15:00 & & $<80$ & $<80$ & $<50$ & 5.39 & $<0.5$ & 24.7 & $<0.1$ & 19.2 & $<1$ & $<1$ & $<1$ & $<1$ & 2.32 & 2.24 & 140 \\
\hline 7/26/2001 15:00 & $\mathrm{R}$ & $<80$ & $<80$ & $<50$ & 5.30 & $<0.5$ & 24.9 & $<0.1$ & 19.5 & $<1$ & $<1$ & $<1$ & $<1$ & 2.38 & 1.88 & 136 \\
\hline 7/26/2001 16:00 & & $<80$ & $<80$ & $<50$ & 5.49 & $<0.5$ & 24.6 & $<0.1$ & 19.5 & $<1$ & $<1$ & $<1$ & $<1$ & 2.22 & 1.99 & 139 \\
\hline $7 / 26 / 2001 \quad 16: 30$ & & $<80$ & $<80$ & $<50$ & -- & -- & 24.3 & $<0.1$ & 19.6 & $<1$ & $<1$ & $<1$ & $<1$ & 2.54 & 2.15 & 146 \\
\hline 7/26/2001 17:00 & & $<80$ & $<80$ & $<50$ & 5.99 & $<0.5$ & 24.7 & $<0.1$ & 18.9 & $<1$ & $<1$ & $<1$ & $<1$ & 2.34 & 2.22 & 152 \\
\hline 7/26/2001 17:30 & & $<80$ & $<80$ & $<50$ & -- & -- & 24.6 & $<0.1$ & 19.2 & $<1$ & $<1$ & $<1$ & $<1$ & 2.61 & 2.01 & 153 \\
\hline 7/26/2001 18:00 & & $<80$ & $<80$ & $<50$ & 5.84 & $<0.5$ & 25.1 & $<0.1$ & 19.0 & $<1$ & $<1$ & $<1$ & $<1$ & 2.51 & 2.30 & 135 \\
\hline 7/26/2001 18:30 & & $<80$ & $<80$ & $<50$ & -- & -- & 25.4 & $<0.1$ & 19.0 & $<1$ & $<1$ & $<1$ & $<1$ & 2.42 & 2.20 & 138 \\
\hline 7/26/2001 19:00 & & $<80$ & $<80$ & $<50$ & 6.11 & $<0.5$ & 25.0 & $<0.1$ & 19.1 & $<1$ & $<1$ & $<1$ & $<1$ & 2.43 & 2.17 & 138 \\
\hline 7/26/2001 19:30 & & $<80$ & $<80$ & $<50$ & 6.03 & $<0.5$ & 25.5 & $<0.1$ & 19.6 & $<1$ & $<1$ & $<1$ & $<1$ & 2.16 & 2.12 & 141 \\
\hline 7/26/2001 20:00 & & $<80$ & $<80$ & $<50$ & 5.36 & 0.93 & 25.6 & $<0.1$ & 19.4 & $<1$ & $<1$ & $<1$ & $<1$ & 2.55 & 2.23 & 145 \\
\hline 7/26/2001 21:00 & & $<80$ & $<80$ & $<50$ & -- & -- & 26.1 & $<0.1$ & 19.6 & $<1$ & $<1$ & $<1$ & $<1$ & 2.64 & 2.12 & 159 \\
\hline 7/26/2001 22:00 & & $<80$ & $<80$ & $<50$ & 4.34 & $<0.5$ & 26.8 & $<0.1$ & 20.2 & $<1$ & $<1$ & $<1$ & $<1$ & 2.87 & 2.59 & 149 \\
\hline 7/26/2001 23:00 & & $<80$ & $<80$ & $<50$ & 5.00 & 0.57 & 27.6 & $<0.1$ & 20.2 & $<1$ & $<1$ & $<1$ & $<1$ & 3.05 & 2.40 & 157 \\
\hline $7 / 27 / 2001 \quad 0: 00$ & & $<80$ & $<80$ & $<50$ & 4.65 & 0.58 & 27.9 & $<0.1$ & 20.3 & $<1$ & $<1$ & $<1$ & $<1$ & 2.32 & 1.95 & 143 \\
\hline $7 / 27 / 2001 \quad 1: 00$ & & $<80$ & $<80$ & $<50$ & 4.55 & 0.50 & 28.2 & $<0.1$ & 20.2 & $<1$ & $<1$ & $<1$ & $<1$ & 2.65 & 2.39 & 146 \\
\hline $7 / 27 / 2001 \quad 2: 00$ & & $<80$ & $<80$ & $<50$ & 4.25 & $<0.5$ & 28.2 & $<0.1$ & 20.3 & $<1$ & $<1$ & $<1$ & $<1$ & 2.22 & 2.44 & 147 \\
\hline $7 / 27 / 2001 \quad 3: 00$ & & $<80$ & $<80$ & $<50$ & 4.15 & $<0.5$ & 28.0 & $<0.1$ & 20.4 & $<1$ & $<1$ & $<1$ & $<1$ & 3.11 & 2.07 & 140 \\
\hline $7 / 27 / 2001 \quad 4: 00$ & & $<80$ & $<80$ & $<50$ & 4.09 & $<0.5$ & 27.9 & $<0.1$ & 20.0 & $<1$ & $<1$ & $<1$ & $<1$ & 2.48 & 2.19 & 149 \\
\hline
\end{tabular}


Table 5. Water-quality data for diel investigations in Prickly Pear Creek at site PP-4b, Montana, July 25-27, 2001 (Continued).

\begin{tabular}{|c|c|c|c|c|c|c|c|c|c|c|c|c|c|c|c|c|c|c|}
\hline Date and time & $\begin{array}{c}\text { QA } \\
\text { sample } \\
\text { type }\end{array}$ & $\begin{array}{c}\text { Iron, } \\
\mathrm{Fe}^{+2}+ \\
\mathrm{Fe}^{+3}, \\
\text { dis- } \\
\text { solved } \\
(\mu \mathrm{g} / \mathrm{L})\end{array}$ & $\begin{array}{c}\text { Iron, } \\
\text { Fe }^{+2}, \\
\text { dis- } \\
\text { solved } \\
(\mu \mathrm{g} / \mathrm{L})\end{array}$ & $\begin{array}{c}\text { Lead, } \\
\text { total } \\
\text { recov- } \\
\text { erable } \\
(\mu \mathrm{g} / \mathrm{L})\end{array}$ & $\begin{array}{c}\text { Lead, } \\
\text { dis- } \\
\text { solved } \\
(\mu \mathrm{g} / \mathrm{L})\end{array}$ & $\begin{array}{c}\text { Lith- } \\
\text { ium, } \\
\text { dis- } \\
\text { solved } \\
(\mu \mathrm{g} / \mathrm{L})\end{array}$ & $\begin{array}{c}\text { Manga- } \\
\text { nese, } \\
\text { total } \\
\text { recov- } \\
\text { erable } \\
(\mu \mathrm{g} / \mathrm{L})\end{array}$ & $\begin{array}{c}\text { Manga- } \\
\text { nese, } \\
\text { dis- } \\
\text { solved } \\
(\mu \mathrm{g} / \mathrm{L})\end{array}$ & $\begin{array}{c}\text { Nickel, } \\
\text { total } \\
\text { recov- } \\
\text { erable } \\
(\mu \mathrm{g} / \mathrm{L})\end{array}$ & $\begin{array}{c}\text { Nickel, } \\
\text { dis- } \\
\text { solved } \\
(\mu \mathrm{g} / \mathrm{L})\end{array}$ & $\begin{array}{c}\text { Sele- } \\
\text { nium, } \\
\text { total } \\
\text { recov- } \\
\text { erable } \\
(\mu \mathrm{g} / \mathrm{L})\end{array}$ & $\begin{array}{c}\text { Sele- } \\
\text { nium, } \\
\text { dis- } \\
\text { solved } \\
(\mu \mathrm{g} / \mathrm{L})\end{array}$ & $\begin{array}{l}\text { Stron- } \\
\text { tium, } \\
\text { total } \\
\text { recov- } \\
\text { erable } \\
(\mu \mathrm{g} / \mathrm{L}) \\
\end{array}$ & $\begin{array}{c}\text { Stron- } \\
\text { tium, } \\
\text { dis- } \\
\text { solved } \\
(\mu \mathrm{g} / \mathrm{L})\end{array}$ & $\begin{array}{c}\text { Vana- } \\
\text { dium, } \\
\text { total } \\
\text { recov- } \\
\text { erable } \\
(\mu \mathrm{g} / \mathrm{L}) \\
\end{array}$ & $\begin{array}{c}\text { Vana- } \\
\text { dium, } \\
\text { dis- } \\
\text { solved } \\
(\mu \mathrm{g} / \mathrm{L})\end{array}$ & $\begin{array}{l}\text { Zinc, } \\
\text { total } \\
\text { recov- } \\
\text { erable } \\
(\mu \mathrm{g} / \mathrm{L})\end{array}$ & $\begin{array}{c}\text { Zinc, } \\
\text { dis- } \\
\text { solved } \\
(\mu \mathrm{g} / \mathrm{L})\end{array}$ \\
\hline $7 / 26 / 2001 \quad 8: 30$ & B & $<2$ & $<2$ & $<8$ & $<8$ & $<8$ & $<1$ & $<1$ & $<2$ & $<2$ & $<50$ & $<50$ & $<1$ & $<1$ & $<1$ & $<1$ & $<1$ & $<1$ \\
\hline 7/26/2001 9:00 & & 47.5 & 30.1 & $<8$ & $<8$ & 22.3 & 60.1 & 51.6 & $<2$ & $<2$ & $<50$ & $<50$ & 246 & 246 & $<1$ & $<1$ & 106 & 98.8 \\
\hline 7/26/2001 10:00 & & 47.2 & 23.8 & $<8$ & $<8$ & 22.0 & 56.6 & 49.0 & $<2$ & $<2$ & $<50$ & $<50$ & 246 & 246 & $<1$ & $<1$ & 96.6 & 89.7 \\
\hline 7/26/2001 11:00 & & 44.8 & 20.7 & $<8$ & $<8$ & 21.0 & 52.9 & 45.9 & $<2$ & $<2$ & $<50$ & $<50$ & 247 & 242 & $<1$ & $<1$ & 85.0 & 73.3 \\
\hline 7/26/2001 12:00 & & 43.2 & 25.7 & $<8$ & $<8$ & 21.5 & 51.4 & 42.8 & $<2$ & $<2$ & $<50$ & $<50$ & 249 & 246 & $<1$ & $<1$ & 76.0 & 62.8 \\
\hline 7/26/2001 13:00 & & 42.4 & 28.8 & $<8$ & $<8$ & 21.1 & 47.3 & 39.4 & $<2$ & $<2$ & $<50$ & $<50$ & 247 & 245 & $<1$ & $<1$ & 63.4 & 51.6 \\
\hline 7/26/2001 14:00 & & 39.4 & 20.9 & $<8$ & $<8$ & 21.1 & 47.7 & 36.9 & $<2$ & $<2$ & $<50$ & $<50$ & 245 & 244 & $<1$ & $<1$ & 55.0 & 41.8 \\
\hline 7/26/2001 15:00 & & 36.1 & 21.8 & $<8$ & $<8$ & 21.1 & 42.9 & 34.6 & $<2$ & $<2$ & $<50$ & $<50$ & 246 & 244 & $<1$ & $<1$ & 46.8 & 35.5 \\
\hline 7/26/2001 15:00 & $\mathrm{R}$ & 36.8 & 24.5 & $<8$ & $<8$ & 20.8 & 43.2 & 34.3 & $<2$ & $<2$ & $<50$ & $<50$ & 245 & 242 & $<1$ & $<1$ & 46.0 & 33.4 \\
\hline 7/26/2001 16:00 & & 36.7 & 13.7 & $<8$ & $<8$ & 21.3 & 41.7 & 33.6 & $<2$ & $<2$ & $<50$ & $<50$ & 245 & 245 & $<1$ & $<1$ & 44.9 & 30.7 \\
\hline $7 / 26 / 2001 \quad 16: 30$ & & 42.9 & -- & $<8$ & $<8$ & 21.3 & 43.1 & 33.1 & $<2$ & $<2$ & $<50$ & $<50$ & 245 & 242 & 1.1 & $<1$ & 44.6 & 30.3 \\
\hline 7/26/2001 17:00 & & 36.5 & 35.8 & $<8$ & $<8$ & 21.6 & 44.2 & 34.0 & $<2$ & $<2$ & $<50$ & $<50$ & 246 & 242 & 1.3 & $<1$ & 45.4 & 29.3 \\
\hline $7 / 26 / 2001 \quad 17: 30$ & & 42.4 & -- & $<8$ & $<8$ & 22.0 & 44.8 & 34.5 & $<2$ & $<2$ & $<50$ & $<50$ & 243 & 242 & 1.3 & $<1$ & 44.9 & 29.6 \\
\hline 7/26/2001 18:00 & & 36.8 & 35.4 & $<8$ & $<8$ & 21.5 & 43.5 & 34.3 & $<2$ & $<2$ & $<50$ & $<50$ & 242 & 247 & 1.2 & $<1$ & 44.7 & 29.2 \\
\hline $7 / 26 / 200118: 30$ & & 45.7 & -- & $<8$ & $<8$ & 22.2 & 44.4 & 35.7 & $<2$ & $<2$ & $<50$ & $<50$ & 244 & 248 & 1.3 & $<1$ & 44.5 & 31.5 \\
\hline 7/26/2001 19:00 & & 40.5 & 40.1 & $<8$ & $<8$ & 22.4 & 46.1 & 36.7 & $<2$ & $<2$ & $<50$ & $<50$ & 245 & 249 & 1.1 & 1.1 & 45.4 & 32.2 \\
\hline $7 / 26 / 2001 \quad 19: 30$ & & 40.1 & 39.5 & $<8$ & $<8$ & 22.2 & 47.3 & 37.7 & $<2$ & $<2$ & $<50$ & $<50$ & 245 & 247 & 1.1 & $<1$ & 46.2 & 33.6 \\
\hline $7 / 26 / 200120: 00$ & & 43.9 & 43.4 & $<8$ & $<8$ & 22.3 & 47.2 & 38.5 & $<2$ & $<2$ & $<50$ & $<50$ & 248 & 248 & 1.3 & $<1$ & 47.4 & 35.0 \\
\hline $7 / 26 / 200121: 00$ & & 46.9 & -- & $<8$ & $<8$ & 22.5 & 52.6 & 42.7 & $<2$ & $<2$ & $<50$ & $<50$ & 249 & 248 & $<1$ & $<1$ & 56.7 & 40.9 \\
\hline $7 / 26 / 200122: 00$ & & 35.8 & 33.6 & $<8$ & $<8$ & 22.7 & 54.0 & 45.7 & $<2$ & $<2$ & $<50$ & $<50$ & 252 & 249 & $<1$ & $<1$ & 62.0 & 49.7 \\
\hline 7/26/2001 23:00 & & 43.8 & 42.4 & $<8$ & $<8$ & 23.5 & 58.5 & 49.9 & $<2$ & $<2$ & $<50$ & $<50$ & 251 & 252 & 1.3 & $<1$ & 73.9 & 61.9 \\
\hline $7 / 27 / 2001 \quad 0: 00$ & & 45.8 & 45.1 & $<8$ & $<8$ & 23.1 & 59.3 & 53.4 & $<2$ & $<2$ & $<50$ & $<50$ & 253 & 253 & 1.0 & $<1$ & 80.7 & 68.0 \\
\hline $7 / 27 / 2001 \quad 1: 00$ & & 44.6 & 42.6 & $<8$ & $<8$ & 23.0 & 61.7 & 53.3 & $<2$ & $<2$ & $<50$ & $<50$ & 252 & 252 & $<1$ & $<1$ & 90.6 & 81.1 \\
\hline $7 / 27 / 2001 \quad 2: 00$ & & 44.5 & 41.3 & $<8$ & $<8$ & 23.7 & 62.7 & 54.5 & $<2$ & $<2$ & $<50$ & $<50$ & 252 & 255 & $<1$ & $<1$ & 100 & 88.9 \\
\hline $7 / 27 / 2001 \quad 3: 00$ & & 41.8 & 39.2 & $<8$ & $<8$ & 23.2 & 63.3 & 55.7 & $<2$ & $<2$ & $<50$ & $<50$ & 249 & 252 & $<1$ & $<1$ & 110 & 97.9 \\
\hline $7 / 27 / 2001 \quad 4: 00$ & & 47.1 & 44.1 & $<8$ & $<8$ & 22.9 & 65.1 & 56.7 & $<2$ & $<2$ & $<50$ & $<50$ & 250 & 253 & $<1$ & $<1$ & 117 & 103 \\
\hline
\end{tabular}


Table 5. Water-quality data for diel investigations in Prickly Pear Creek at site PP-4b, Montana, July 25-27, 2001(Continued).

\begin{tabular}{|c|c|c|c|c|c|c|c|c|c|c|c|c|c|c|c|c|}
\hline Date and time & $\begin{array}{c}\text { QA } \\
\text { sample } \\
\text { type }\end{array}$ & $\begin{array}{c}\text { Stream- } \\
\text { flow, } \\
\text { instan- } \\
\text { taneous } \\
\left(\mathbf{f t}^{3} / \mathbf{s}\right)\end{array}$ & $\begin{array}{l}\text { Specific } \\
\text { conduct- } \\
\text { ance, field } \\
(\mu \mathrm{S} / \mathrm{cm}) \\
\end{array}$ & $\begin{array}{l}\mathrm{pH}, \\
\text { field } \\
\text { (su) }\end{array}$ & $\begin{array}{c}\text { Tempera- } \\
\text { ture, } \\
\text { water } \\
\left({ }^{\circ} \mathrm{C}\right)\end{array}$ & $\begin{array}{c}\text { Dis- } \\
\text { solved } \\
\text { oxygen, } \\
\text { field } \\
(\mathrm{mg} / \mathrm{L})\end{array}$ & $\begin{array}{c}\text { Cal- } \\
\text { cium, } \\
\text { dis- } \\
\text { solved } \\
(\mathrm{mg} / \mathrm{L})\end{array}$ & $\begin{array}{l}\text { Magne- } \\
\text { sium, } \\
\text { dissolved } \\
(\mathrm{mg} / \mathrm{L})\end{array}$ & $\begin{array}{c}\text { Sodium, } \\
\text { dis- } \\
\text { solved } \\
(\mathrm{mg} / \mathrm{L})\end{array}$ & $\begin{array}{l}\text { Potas- } \\
\text { sium, } \\
\text { dis- } \\
\text { solved } \\
(\mathrm{mg} / \mathrm{L})\end{array}$ & $\begin{array}{c}\text { Alka- } \\
\text { linity, } \\
\text { lab } \\
(\mathbf{m g} / \mathrm{L} \text { as } \\
\left.\mathrm{CaCO}_{3}\right)\end{array}$ & $\begin{array}{c}\text { Sulfate, } \\
\text { dis- } \\
\text { solved } \\
(\text { mg as } \\
\left.\mathrm{SO}_{4}\right)\end{array}$ & $\begin{array}{c}\text { Chloride, } \\
\text { dissolved } \\
(\mathrm{mg} / \mathrm{L})\end{array}$ & $\begin{array}{c}\text { Fluo- } \\
\text { ride, } \\
\text { dis- } \\
\text { solved } \\
(\mathrm{mg} / \mathrm{L})\end{array}$ & 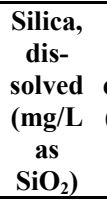 & $\begin{array}{c}\text { Nitrate, } \\
\text { dissolved } \\
(\mathrm{mg} / \mathrm{L} \text { as } \\
\left.\mathrm{NO}_{3}\right)\end{array}$ \\
\hline $7 / 27 / 2001 \quad 5: 00$ & & -- & 244 & 8.14 & 12.71 & 8.66 & 27.5 & 6.10 & 15.5 & 2.35 & 89.3 & 41.8 & 2.99 & 0.45 & 21.5 & 0.67 \\
\hline $7 / 27 / 2001 \quad 5: 30$ & & 17.4 & 244 & 8.14 & 12.42 & 8.72 & 27.0 & 5.93 & 15.0 & 2.31 & 88.0 & 41.1 & 2.92 & 0.42 & 21.4 & 0.60 \\
\hline $7 / 27 / 2001 \quad 6: 00$ & & -- & 244 & 8.15 & 12.16 & 8.92 & 26.9 & 5.91 & 14.9 & 2.32 & 89.7 & 42.2 & 3.05 & 0.47 & 21.2 & 0.62 \\
\hline $7 / 27 / 2001 \quad 6: 30$ & & 17.8 & 244 & 8.16 & 11.90 & 9.12 & 27.1 & 6.01 & 15.2 & 2.34 & 88.3 & 41.8 & 2.96 & 0.42 & 21.7 & 0.67 \\
\hline $7 / 27 / 2001 \quad 7: 00$ & & -- & 244 & 8.17 & 11.72 & 9.25 & 26.9 & 6.01 & 15.0 & 2.32 & 88.7 & 42.3 & 2.95 & 0.43 & 21.1 & 0.69 \\
\hline $7 / 27 / 2001 \quad 7: 30$ & & 17.8 & 243 & 8.18 & 11.63 & 9.41 & 27.0 & 5.95 & 14.9 & 2.27 & 88.2 & 42.1 & 2.95 & 0.42 & 21.2 & 0.62 \\
\hline $7 / 27 / 2001 \quad 8: 00$ & & -- & 243 & 8.19 & 11.64 & 9.52 & 27.1 & 5.98 & 15.3 & 2.32 & 89.5 & 42.6 & 3.04 & 0.43 & 20.8 & 0.67 \\
\hline $7 / 27 / 2001 \quad 8: 30$ & & 18.2 & 242 & 8.21 & 11.78 & 9.60 & 26.6 & 5.83 & 14.9 & 2.34 & 88.5 & 41.7 & 2.95 & 0.44 & 21.2 & 0.57 \\
\hline $7 / 27 / 2001 \quad 9: 00$ & & 17.8 & 242 & 8.24 & 12.03 & 9.66 & 26.6 & 6.11 & 15.0 & 2.31 & 89.1 & 42.0 & 2.97 & 0.42 & 21.2 & 0.65 \\
\hline $7 / 27 / 2001$ 10:00 & & -- & 242 & -- & 12.91 & 9.61 & 26.5 & 6.06 & 15.1 & 2.27 & 89.1 & 42.4 & 2.95 & 0.46 & 21.4 & 0.67 \\
\hline
\end{tabular}


Table 6. Water-quality data for diel investigations in Prickly Pear Creek at site PP-5, Montana, July 25-27, 2001.

[Samples analyzed by the U.S. Geological Survey laboratory, Boulder, Colo. Analytical data are reported to a maximum of three significant figures to provide sufficient resolution for detecting small differences between samples closely spaced in time or distance. Similarly, field measurements from electronic recorders have not been rounded to illustrate the pattern of relative change between

measurements. Abbreviations: B, blank sample; $\mathrm{ft}^{3} / \mathrm{s}$, cubic feet per second; ${ }^{\circ} \mathrm{C}$, degrees Celsius, $\mu \mathrm{g} / \mathrm{L}$, micrograms per liter; $\mu \mathrm{S} / \mathrm{cm}$, microsiemens per centimeter at $25^{\circ} \mathrm{C} ; \mathrm{mg} / \mathrm{L}$, milligrams per liter; su, standard units; QA, quality assurance; R, replicate sample. Symbol: $<$, less than; --, no data].

\begin{tabular}{|c|c|c|c|c|c|c|c|c|c|c|c|c|c|c|c|c|}
\hline Date and time & $\begin{array}{c}\text { QA } \\
\text { sample } \\
\text { type }\end{array}$ & $\begin{array}{c}\text { Stream- } \\
\text { flow, } \\
\text { instan- } \\
\text { taneous } \\
\left(\mathbf{f t}^{3} / \mathbf{s}\right)\end{array}$ & 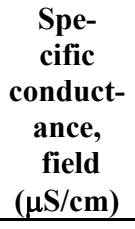 & $\begin{array}{l}\mathrm{pH}, \\
\text { field } \\
\text { (su) }\end{array}$ & $\begin{array}{l}\text { Tem- } \\
\text { pera- } \\
\text { ture, } \\
\text { water } \\
\left({ }^{\circ} \mathrm{C}\right)\end{array}$ & $\begin{array}{c}\text { Dis- } \\
\text { solved } \\
\text { oxygen, } \\
\text { field } \\
(\mathrm{mg} / \mathrm{L})\end{array}$ & $\begin{array}{c}\text { Cal- } \\
\text { cium, } \\
\text { dis- } \\
\text { solved } \\
(\mathrm{mg} / \mathrm{L})\end{array}$ & $\begin{array}{l}\text { Magne- } \\
\text { sium, } \\
\text { dissolved } \\
(\mathrm{mg} / \mathrm{L})\end{array}$ & $\begin{array}{c}\text { So- } \\
\text { dium, } \\
\text { dis- } \\
\text { solved } \\
(\mathrm{mg} / \mathrm{L})\end{array}$ & $\begin{array}{l}\text { Potas- } \\
\text { sium, } \\
\text { dis- } \\
\text { solved } \\
(\mathrm{mg} / \mathrm{L})\end{array}$ & $\begin{array}{c}\text { Alka- } \\
\text { linity, } \\
\text { lab } \\
(\mathrm{mg} / \mathrm{L} \text { as } \\
\left.\mathrm{CaCO}_{3}\right)\end{array}$ & $\begin{array}{c}\text { Sulfate, } \\
\text { dis- } \\
\text { solved } \\
(\mathrm{mg} \text { as } \\
\left.\mathrm{SO}_{4}\right)\end{array}$ & $\begin{array}{c}\text { Chlo- } \\
\text { ride, } \\
\text { dis- } \\
\text { solved } \\
(\mathrm{mg} / \mathrm{L})\end{array}$ & $\begin{array}{c}\text { Fluo- } \\
\text { ride, } \\
\text { dis- } \\
\text { solved } \\
(\mathrm{mg} / \mathrm{L})\end{array}$ & $\begin{array}{c}\text { Silica, } \\
\text { dis- } \\
\text { solved } \\
(\mathrm{mg} / \mathrm{L} \text { as } \\
\left.\mathrm{SiO}_{2}\right)\end{array}$ & $\begin{array}{c}\text { Nitrate, } \\
\text { dis- } \\
\text { solved } \\
(\mathrm{mg} / \mathrm{L} \text { as } \\
\left.\mathrm{NO}_{3}\right)\end{array}$ \\
\hline 7/25/2001 12:07 & & 19.4 & 244 & 8.34 & 16.60 & 9.01 & 27.1 & 6.43 & 15.0 & 2.30 & 89.8 & 42.7 & 3.26 & 0.39 & 21.2 & 0.68 \\
\hline 7/25/2001 13:00 & & 19.4 & 245 & 8.41 & 18.48 & 8.77 & 27.2 & 6.40 & 14.8 & 2.30 & 89.1 & 42.4 & 3.26 & 0.36 & 21.2 & 0.56 \\
\hline 7/25/2001 14:00 & & 19.0 & 246 & 8.46 & 20.17 & 8.54 & 27.1 & 6.41 & 14.8 & 2.34 & 88.8 & 41.6 & 3.35 & 0.38 & 21.2 & 0.49 \\
\hline 7/25/2001 14:01 & $\mathrm{R}$ & -- & -- & -- & -- & -- & 27.1 & 6.35 & 14.7 & 2.30 & 89.4 & 42.9 & 3.32 & 0.44 & 21.2 & 0.37 \\
\hline 7/25/2001 15:00 & & 19.0 & 246 & 8.48 & 21.54 & 8.22 & 27.2 & 6.39 & 14.8 & 2.35 & 88.2 & 42.6 & 3.32 & 0.42 & 21.4 & 0.62 \\
\hline 7/25/2001 16:00 & & 18.1 & 246 & 8.50 & 22.14 & 7.99 & 27.0 & 6.36 & 14.8 & 2.35 & 87.8 & 41.8 & 3.40 & 0.35 & 21.1 & 0.43 \\
\hline 7/25/2001 17:00 & & 18.6 & 246 & 8.49 & 21.99 & 7.76 & 27.1 & 6.34 & 14.8 & 2.38 & 87.8 & 43.5 & 3.27 & 0.35 & 21.2 & 0.40 \\
\hline 7/25/2001 18:00 & & 17.7 & 246 & 8.44 & 21.65 & 7.63 & 26.9 & 6.37 & 15.0 & 2.39 & 88.0 & 42.9 & 3.28 & 0.36 & 21.3 & 0.37 \\
\hline 7/25/2001 19:00 & & 17.7 & 246 & 8.29 & 21.09 & 7.39 & 26.8 & 6.38 & 14.9 & 2.43 & 88.2 & 43.8 & 3.36 & 0.38 & 21.4 & 0.37 \\
\hline 7/25/2001 20:00 & & 17.3 & 247 & 8.26 & 20.53 & 7.41 & 27.2 & 6.41 & 15.2 & 2.43 & 88.6 & 43.5 & 3.44 & 0.43 & 21.4 & 0.46 \\
\hline 7/25/2001 21:00 & & 17.3 & 248 & 8.17 & 20.01 & 7.38 & 26.9 & 6.33 & 15.0 & 2.45 & 88.7 & 43.6 & 3.36 & 0.43 & 21.4 & 0.46 \\
\hline 7/25/2001 22:00 & & 17.3 & 245 & 8.09 & 19.30 & 7.39 & 27.4 & 6.51 & 15.2 & 2.45 & 89.6 & 44.6 & 3.43 & 0.46 & 21.2 & 0.46 \\
\hline 7/25/2001 23:00 & & 16.9 & -- & 8.05 & 18.46 & 7.48 & 27.6 & 6.53 & 15.2 & 2.45 & 90.1 & 44.5 & 3.40 & 0.44 & 21.3 & 0.55 \\
\hline $7 / 26 / 2001 \quad 0: 00$ & & 16.9 & 254 & 8.07 & 17.67 & 7.61 & 27.5 & 6.54 & 15.3 & 2.47 & 91.0 & 44.5 & 3.44 & 0.40 & 21.3 & 0.53 \\
\hline $7 / 26 / 2001 \quad 1: 00$ & & 16.9 & 252 & 8.05 & 17.03 & 7.75 & 27.7 & 6.59 & 15.3 & 2.49 & 91.7 & 43.8 & 3.41 & 0.45 & 21.4 & 0.50 \\
\hline $7 / 26 / 2001 \quad 2: 00$ & & 17.3 & 252 & 8.05 & 16.43 & 7.89 & 27.9 & 6.52 & 15.7 & 2.51 & 92.2 & 43.3 & 3.49 & 0.46 & 21.1 & 0.62 \\
\hline $7 / 26 / 2001 \quad 3: 00$ & & 16.5 & 253 & 8.04 & 15.94 & 8.05 & 27.9 & 6.69 & 15.7 & 2.48 & 92.7 & 44.7 & 3.51 & 0.37 & 21.2 & 0.71 \\
\hline $7 / 26 / 2001 \quad 4: 00$ & & 17.3 & 253 & 8.01 & 15.31 & 8.16 & 27.8 & 6.65 & 15.7 & 2.46 & 93.0 & 44.5 & 3.58 & 0.45 & 21.4 & 0.68 \\
\hline $7 / 26 / 2001 \quad 5: 00$ & & 16.9 & 253 & 8.02 & 14.63 & 8.37 & 28.0 & 6.66 & 15.7 & 2.44 & 93.2 & 44.5 & 3.48 & 0.47 & 21.4 & 0.71 \\
\hline $7 / 26 / 2001 \quad 6: 00$ & & 17.3 & 253 & 8.01 & 13.95 & 8.48 & 28.0 & 6.65 & 15.8 & 2.45 & 93.2 & 44.4 & 3.47 & 0.38 & 21.2 & 0.74 \\
\hline $7 / 26 / 2001 \quad 7: 00$ & & 17.7 & 252 & 8.00 & 13.32 & 8.76 & 27.9 & 6.66 & 15.8 & 2.44 & 93.4 & 43.5 & 3.30 & 0.31 & 21.4 & 0.85 \\
\hline $7 / 26 / 2001 \quad 8: 00$ & & 17.3 & 251 & 8.06 & 12.86 & 8.97 & 27.8 & 6.58 & 15.9 & 2.40 & 93.0 & 42.9 & 3.30 & 0.32 & 21.3 & 0.63 \\
\hline
\end{tabular}


Table 6. Water-quality data for diel investigations in Prickly Pear Creek at site PP-5, Montana, July 25-27, 2001 (Continued).

\begin{tabular}{|c|c|c|c|c|c|c|c|c|c|c|c|c|c|c|c|c|}
\hline Date and time & $\begin{array}{c}\text { QA } \\
\text { sample } \\
\text { type }\end{array}$ & $\begin{array}{c}\text { Alum- } \\
\text { inum, } \\
\text { total } \\
\text { recov- } \\
\text { erable } \\
(\mu \mathrm{g} / \mathrm{L})\end{array}$ & $\begin{array}{c}\text { Alum- } \\
\text { inum, } \\
\text { dis- } \\
\text { solved } \\
(\mu \mathrm{g} / \mathrm{L})\end{array}$ & $\begin{array}{c}\text { Arsenic, } \\
\text { total } \\
\text { recov- } \\
\text { erable } \\
(\mu \mathrm{g} / \mathrm{L})\end{array}$ & $\begin{array}{c}\text { Arsenic, } \\
\text { total, } \\
\text { dis- } \\
\text { solved } \\
(\mu \mathrm{g} / \mathrm{L})\end{array}$ & $\begin{array}{c}\text { Arsenic, } \\
\text { As }^{+3} \text {, dis- } \\
\text { solved } \\
(\mu \mathrm{g} / \mathrm{L})\end{array}$ & $\begin{array}{c}\text { Barium, } \\
\text { dis- } \\
\text { solved } \\
(\mu \mathrm{g} / \mathrm{L})\end{array}$ & $\begin{array}{c}\text { Beryl- } \\
\text { lium, } \\
\text { dis- } \\
\text { solved } \\
(\mu \mathrm{g} / \mathrm{L})\end{array}$ & $\begin{array}{c}\text { Boron, } \\
\text { dis- } \\
\text { solved } \\
(\mu \mathrm{g} / \mathrm{L})\end{array}$ & $\begin{array}{c}\text { Cad- } \\
\text { mium, } \\
\text { total } \\
\text { recov- } \\
\text { erable } \\
(\mu \mathrm{g} / \mathrm{L}) \\
\end{array}$ & $\begin{array}{c}\text { Cad- } \\
\text { mium, } \\
\text { dis- } \\
\text { solved } \\
(\mu \mathrm{g} / \mathrm{L})\end{array}$ & $\begin{array}{c}\text { Chro- } \\
\text { mium, } \\
\text { dis- } \\
\text { solved } \\
(\mu \mathrm{g} / \mathrm{L})\end{array}$ & $\begin{array}{c}\text { Cobalt, } \\
\text { dis- } \\
\text { solved } \\
(\mu \mathrm{g} / \mathrm{L})\end{array}$ & $\begin{array}{c}\text { Copper, } \\
\text { total } \\
\text { recov- } \\
\text { erable } \\
(\mu \mathrm{g} / \mathrm{L})\end{array}$ & $\begin{array}{c}\text { Copper, } \\
\text { dis- } \\
\text { solved } \\
(\mu \mathrm{g} / \mathrm{L})\end{array}$ & $\begin{array}{l}\text { Iron, } \\
\text { total } \\
\text { recov- } \\
\text { erable } \\
(\mu \mathrm{g} / \mathrm{L})\end{array}$ \\
\hline $7 / 25 / 2001 \quad 12: 07$ & & $<80$ & $<80$ & $<50$ & 4.56 & $<0.5$ & 24.8 & $<0.1$ & 19.8 & $<1$ & $<1$ & $<1$ & 1.30 & 2.71 & 2.44 & 116 \\
\hline 7/25/2001 13:00 & & $<80$ & $<80$ & $<50$ & 4.74 & $<0.5$ & 24.5 & $<0.1$ & 20.2 & $<1$ & $<1$ & $<1$ & $<1$ & 3.23 & 2.34 & 126 \\
\hline 7/25/2001 14:00 & & $<80$ & $<80$ & $<50$ & 4.87 & $<0.5$ & 24.7 & $<0.1$ & 20.2 & $<1$ & $<1$ & $<1$ & $<1$ & 2.80 & 2.15 & 122 \\
\hline 7/25/2001 14:01 & $\mathrm{R}$ & $<80$ & $<80$ & $<50$ & 4.90 & $<0.5$ & 24.7 & $<0.1$ & 20.0 & $<1$ & $<1$ & $<1$ & $<1$ & 3.15 & 2.24 & 131 \\
\hline 7/25/2001 15:00 & & $<80$ & $<80$ & $<50$ & 4.93 & $<0.5$ & 24.4 & $<0.1$ & 20.2 & $<1$ & $<1$ & $<1$ & $<1$ & 3.09 & 2.38 & 121 \\
\hline 7/25/2001 16:00 & & $<80$ & $<80$ & $<50$ & 5.13 & $<0.5$ & 23.8 & $<0.1$ & 20.0 & $<1$ & $<1$ & $<1$ & $<1$ & 2.77 & 2.15 & 122 \\
\hline 7/25/2001 17:00 & & $<80$ & $<80$ & $<50$ & 5.42 & 0.81 & 24.1 & $<0.1$ & 20.2 & $<1$ & $<1$ & $<1$ & $<1$ & 2.73 & 2.54 & 123 \\
\hline 7/25/2001 18:00 & & $<80$ & $<80$ & $<50$ & 5.52 & 0.58 & 25.6 & $<0.1$ & 20.4 & $<1$ & $<1$ & $<1$ & 2.1 & 2.89 & 2.40 & 118 \\
\hline 7/25/2001 19:00 & & $<80$ & $<80$ & $<50$ & 5.55 & $<0.5$ & 26.0 & $<0.1$ & 20.4 & $<1$ & $<1$ & $<1$ & 1.1 & 2.81 & 2.71 & 121 \\
\hline $7 / 25 / 200120: 00$ & & $<80$ & $<80$ & $<50$ & 5.61 & $<0.5$ & 25.6 & $<0.1$ & 20.8 & $<1$ & $<1$ & $<1$ & 1.2 & 2.76 & 2.27 & 119 \\
\hline 7/25/2001 21:00 & & $<80$ & $<80$ & $<50$ & 5.54 & $<0.5$ & 26.6 & $<0.1$ & 21.1 & $<1$ & $<1$ & $<1$ & 1.4 & 2.10 & 2.48 & 123 \\
\hline $7 / 25 / 200122: 00$ & & $<80$ & $<80$ & $<50$ & 5.42 & $<0.5$ & 27.5 & $<0.1$ & 21.7 & $<1$ & $<1$ & $<1$ & 1.7 & 3.07 & 2.57 & 128 \\
\hline 7/25/2001 23:00 & & $<80$ & $<80$ & $<50$ & 5.31 & 1.28 & 27.9 & $<0.1$ & 21.6 & $<1$ & $<1$ & $<1$ & 2.2 & 2.94 & 2.49 & 130 \\
\hline $7 / 26 / 2001 \quad 0: 00$ & & $<80$ & $<80$ & $<50$ & 5.35 & 0.92 & 27.8 & $<0.1$ & 21.8 & $<1$ & $<1$ & $<1$ & $<1$ & 3.12 & 3.15 & 174 \\
\hline $7 / 26 / 2001 \quad 1: 00$ & & $<80$ & $<80$ & $<50$ & 5.04 & 0.98 & 28.3 & $<0.1$ & 21.7 & $<1$ & $<1$ & $<1$ & $<1$ & 2.99 & 2.79 & 157 \\
\hline $7 / 26 / 2001 \quad 2: 00$ & & $<80$ & $<80$ & $<50$ & 4.97 & 1.35 & 28.5 & $<0.1$ & 21.5 & $<1$ & $<1$ & $<1$ & $<1$ & 2.80 & 2.63 & 167 \\
\hline $7 / 26 / 2001 \quad 3: 00$ & & $<80$ & $<80$ & $<50$ & 5.04 & 1.73 & 29.1 & $<0.1$ & 21.9 & $<1$ & $<1$ & $<1$ & 2.2 & 2.86 & 2.42 & 167 \\
\hline $7 / 26 / 2001 \quad 4: 00$ & & $<80$ & $<80$ & $<50$ & 4.79 & 0.77 & 28.7 & $<0.1$ & 21.3 & $<1$ & $<1$ & $<1$ & $<1$ & 2.59 & 2.49 & 143 \\
\hline $7 / 26 / 2001 \quad 5: 00$ & & $<80$ & $<80$ & $<50$ & 4.65 & 1.42 & 29.0 & $<0.1$ & 21.8 & $<1$ & $<1$ & $<1$ & $<1$ & 2.42 & 2.35 & 127 \\
\hline $7 / 26 / 2001 \quad 6: 00$ & & $<80$ & $<80$ & $<50$ & 4.68 & 1.13 & 28.6 & $<0.1$ & 21.9 & $<1$ & $<1$ & $<1$ & $<1$ & 2.50 & 2.63 & 123 \\
\hline $7 / 26 / 2001 \quad 7: 00$ & & $<80$ & $<80$ & $<50$ & 4.49 & $<0.5$ & 28.4 & $<0.1$ & 21.9 & $<1$ & $<1$ & $<1$ & 1.0 & 2.42 & 1.97 & 119 \\
\hline $7 / 26 / 2001 \quad 8: 00$ & & $<80$ & $<80$ & $<50$ & 4.23 & $<0.5$ & 28.2 & $<0.1$ & 20.9 & $<1$ & $<1$ & $<1$ & 1.0 & 2.66 & 2.22 & 133 \\
\hline
\end{tabular}


Table 6. Water-quality data for diel investigations in Prickly Pear Creek at site PP-5, Montana, July 25-27, 2001 (Continued).

\begin{tabular}{|c|c|c|c|c|c|c|c|c|c|c|c|c|c|c|c|c|c|c|}
\hline Date and time & $\begin{array}{c}\text { QA } \\
\text { sample } \\
\text { type }\end{array}$ & $\begin{array}{c}\text { Iron, } \\
\text { total, } \\
\text { dis- } \\
\text { solved } \\
(\mu \mathrm{g} / \mathrm{L})\end{array}$ & $\begin{array}{c}\text { Iron, } \\
\mathrm{Fe}^{+2}, \\
\text { dis- } \\
\text { solved } \\
(\mu \mathrm{g} / \mathrm{L})\end{array}$ & $\begin{array}{l}\text { Lead, } \\
\text { total } \\
\text { recov- } \\
\text { erable } \\
(\mu \mathrm{g} / \mathrm{L})\end{array}$ & $\begin{array}{c}\text { Lead, } \\
\text { dis- } \\
\text { solved } \\
(\mu \mathrm{g} / \mathrm{L})\end{array}$ & $\begin{array}{l}\text { Lith- } \\
\text { ium, } \\
\text { dis- } \\
\text { solved } \\
(\mu \mathrm{g} / \mathrm{L})\end{array}$ & $\begin{array}{c}\text { Manga- } \\
\text { nese, } \\
\text { total } \\
\text { recov- } \\
\text { erable } \\
(\mu \mathrm{g} / \mathrm{L}) \\
\end{array}$ & $\begin{array}{c}\text { Manga- } \\
\text { nese, } \\
\text { dis- } \\
\text { solved } \\
(\mu \mathrm{g} / \mathrm{L})\end{array}$ & $\begin{array}{c}\text { Nickel, } \\
\text { total } \\
\text { recov- } \\
\text { erable } \\
(\mu \mathrm{g} / \mathrm{L})\end{array}$ & $\begin{array}{c}\text { Nickel, } \\
\text { dis- } \\
\text { solved } \\
(\mu \mathrm{g} / \mathrm{L})\end{array}$ & 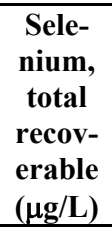 & $\begin{array}{c}\text { Sele- } \\
\text { nium, } \\
\text { dis- } \\
\text { solved } \\
(\mu \mathrm{g} / \mathrm{L})\end{array}$ & $\begin{array}{c}\text { Stron- } \\
\text { tium, } \\
\text { total } \\
\text { recov- } \\
\text { erable } \\
(\mu \mathrm{g} / \mathrm{L}) \\
\end{array}$ & $\begin{array}{c}\text { Stron- } \\
\text { tium, } \\
\text { dis- } \\
\text { solved } \\
(\mu \mathrm{g} / \mathrm{L})\end{array}$ & $\begin{array}{l}\text { Vana- } \\
\text { dium, } \\
\text { total } \\
\text { recov- } \\
\text { erable } \\
(\mu \mathrm{g} / \mathrm{L}) \\
\end{array}$ & $\begin{array}{c}\text { Vana- } \\
\text { dium, } \\
\text { dis- } \\
\text { solved } \\
(\mu \mathrm{g} / \mathrm{L})\end{array}$ & $\begin{array}{l}\text { Zinc, } \\
\text { total } \\
\text { recov- } \\
\text { erable } \\
(\mu \mathrm{g} / \mathrm{L})\end{array}$ & $\begin{array}{c}\text { Zinc, } \\
\text { dis- } \\
\text { solved } \\
(\mu \mathrm{g} / \mathrm{L})\end{array}$ \\
\hline $7 / 25 / 2001 \quad 12: 07$ & & 25.0 & 22.9 & $<8$ & $<8$ & 20.6 & 38.6 & 26.6 & $<2$ & $<2$ & $<50$ & $<50$ & 243 & 239 & $<1$ & $<1$ & 55.8 & 42.8 \\
\hline 7/25/2001 13:00 & & 25.3 & 22.3 & $<8$ & $<8$ & 20.2 & 38.2 & 25.3 & $<2$ & $<2$ & $<50$ & $<50$ & 245 & 239 & $<1$ & $<1$ & 51.4 & 27.1 \\
\hline 7/25/2001 14:00 & & 18.0 & 17.1 & $<8$ & $<8$ & 20.5 & 37.2 & 23.4 & $<2$ & $<2$ & $<50$ & $<50$ & 244 & 241 & 1.0 & $<1$ & 45.3 & 20.3 \\
\hline 7/25/2001 14:01 & $\mathrm{R}$ & 18.0 & 17.8 & $<8$ & $<8$ & 20.1 & 38.1 & 23.6 & $<2$ & $<2$ & $<50$ & $<50$ & 245 & 241 & $<1$ & $<1$ & 45.5 & 20.9 \\
\hline 7/25/2001 15:00 & & 15.1 & 14.2 & $<8$ & $<8$ & 20.6 & 37.2 & 22.2 & $<2$ & $<2$ & $<50$ & $<50$ & 244 & 242 & $<1$ & $<1$ & 43.1 & 16.5 \\
\hline 7/25/2001 16:00 & & 15.6 & 15.6 & $<8$ & $<8$ & 20.4 & 35.4 & 20.9 & $<2$ & $<2$ & $<50$ & $<50$ & 244 & 238 & 1.2 & $<1$ & 40.1 & 13.2 \\
\hline 7/25/2001 17:00 & & 15.3 & 14.7 & $<8$ & $<8$ & 20.4 & 34.6 & 20.8 & 2.1 & $<2$ & $<50$ & $<50$ & 243 & 240 & $<1$ & $<1$ & 37.9 & 13.1 \\
\hline 7/25/2001 18:00 & & 22.4 & 20.7 & $<8$ & $<8$ & 20.6 & 34.6 & 20.8 & $<2$ & $<2$ & $<50$ & $<50$ & 243 & 239 & $<1$ & $<1$ & 38.8 & 14.3 \\
\hline 7/25/2001 19:00 & & 15.2 & 14.4 & $<8$ & $<8$ & 20.3 & 36.6 & 21.5 & $<2$ & $<2$ & $<50$ & $<50$ & 243 & 242 & 1.0 & $<1$ & 40.8 & 15.6 \\
\hline 7/25/2001 20:00 & & 16.9 & 16.4 & $<8$ & $<8$ & 20.5 & 36.7 & 22.9 & $<2$ & $<2$ & $<50$ & $<50$ & 245 & 243 & $<1$ & $<1$ & 42.1 & 19.0 \\
\hline 7/25/2001 21:00 & & 19.4 & 19.4 & $<8$ & $<8$ & 20.7 & 39.0 & 23.9 & $<2$ & $<2$ & $<50$ & $<50$ & 245 & 243 & 1.2 & $<1$ & 50.2 & 24.9 \\
\hline 7/25/2001 22:00 & & 15.6 & 13.6 & $<8$ & $<8$ & 20.7 & 40.7 & 25.7 & $<2$ & $<2$ & $<50$ & $<50$ & 247 & 245 & $<1$ & $<1$ & 52.4 & 29.2 \\
\hline 7/25/2001 23:00 & & 24.6 & 23.1 & $<8$ & $<8$ & 20.8 & 41.4 & 26.3 & $<2$ & $<2$ & $<50$ & $<50$ & 250 & 246 & 1.1 & $<1$ & 54.9 & 30.6 \\
\hline $7 / 26 / 2001 \quad 0: 00$ & & 25.4 & 24.8 & $<8$ & $<8$ & 21.4 & 49.9 & 31.5 & $<2$ & $<2$ & $<50$ & $<50$ & 251 & 249 & $<1$ & $<1$ & 62.1 & 34.2 \\
\hline $7 / 26 / 2001 \quad 1: 00$ & & 27.1 & 26.8 & $<8$ & $<8$ & 21.5 & 47.5 & 30.0 & 2.0 & $<2$ & $<50$ & $<50$ & 254 & 252 & $<1$ & $<1$ & 64.8 & 37.3 \\
\hline $7 / 26 / 2001 \quad 2: 00$ & & 31.3 & 30.4 & $<8$ & $<8$ & 21.9 & 47.9 & 30.7 & $<2$ & $<2$ & $<50$ & $<50$ & 254 & 253 & $<1$ & $<1$ & 67.8 & 41.2 \\
\hline $7 / 26 / 2001 \quad 3: 00$ & & 30.8 & 28.2 & $<8$ & $<8$ & 21.6 & 49.3 & 33.4 & $<2$ & $<2$ & $<50$ & $<50$ & 254 & 253 & $<1$ & $<1$ & 69.8 & 40.6 \\
\hline $7 / 26 / 2001 \quad 4: 00$ & & 31.0 & 30.5 & $<8$ & $<8$ & 21.9 & 45.9 & 32.2 & $<2$ & $<2$ & $<50$ & $<50$ & 251 & 251 & $<1$ & $<1$ & 68.7 & 45.1 \\
\hline $7 / 26 / 2001 \quad 5: 00$ & & 31.6 & 29.5 & $<8$ & $<8$ & 21.9 & 45.1 & 30.3 & $<2$ & $<2$ & $<50$ & $<50$ & 255 & 254 & $<1$ & $<1$ & 68.4 & 47.1 \\
\hline $7 / 26 / 2001 \quad 6: 00$ & & 31.6 & 29.7 & $<8$ & $<8$ & 22.2 & 43.8 & 30.8 & $<2$ & $<2$ & $<50$ & $<50$ & 251 & 251 & $<1$ & $<1$ & 73.5 & 51.5 \\
\hline $7 / 26 / 2001 \quad 7: 00$ & & 31.8 & 29.2 & $<8$ & $<8$ & 22.2 & 42.8 & 30.9 & $<2$ & $<2$ & $<50$ & $<50$ & 250 & 253 & $<1$ & $<1$ & 72.9 & 52.1 \\
\hline $7 / 26 / 2001 \quad 8: 00$ & & 30.7 & 28.5 & $<8$ & $<8$ & 22.3 & 43.6 & 31.8 & $<2$ & $<2$ & $<50$ & $<50$ & 251 & 251 & $<1$ & $<1$ & 75.3 & 52.4 \\
\hline
\end{tabular}


Table 6. Water-quality data for diel investigations in Prickly Pear Creek at site PP-5, Montana, July 25-27, 2001 (Continued).

\begin{tabular}{|c|c|c|c|c|c|c|c|c|c|c|c|c|c|c|c|c|}
\hline Date and time & $\begin{array}{c}\text { QA } \\
\text { sample } \\
\text { type }\end{array}$ & $\begin{array}{l}\text { Stream- } \\
\text { flow, } \\
\text { instan- } \\
\text { taneous } \\
\left(\mathbf{f t}^{3} / \mathbf{s}\right)\end{array}$ & $\begin{array}{c}\text { Spe- } \\
\text { cific } \\
\text { conduct- } \\
\text { ance, } \\
\text { field } \\
(\mu \mathrm{S} / \mathrm{cm}) \\
\end{array}$ & $\begin{array}{c}\text { pH, } \\
\text { field } \\
\text { (su) }\end{array}$ & $\begin{array}{l}\text { Tem- } \\
\text { pera- } \\
\text { ture, } \\
\text { water } \\
\left({ }^{\circ} \mathrm{C}\right)\end{array}$ & $\begin{array}{c}\text { Dis- } \\
\text { solved } \\
\text { oxygen, } \\
\text { field } \\
(\mathrm{mg} / \mathrm{L})\end{array}$ & $\begin{array}{c}\text { Cal- } \\
\text { cium, } \\
\text { dis- } \\
\text { solved } \\
(\mathbf{m g} / \mathbf{L})\end{array}$ & $\begin{array}{l}\text { Magne- } \\
\text { sium, } \\
\text { dissolved } \\
(\mathrm{mg} / \mathrm{L})\end{array}$ & $\begin{array}{c}\text { So- } \\
\text { dium, } \\
\text { dis- } \\
\text { solved } \\
(\mathrm{mg} / \mathrm{L})\end{array}$ & $\begin{array}{l}\text { Potas- } \\
\text { sium, } \\
\text { dis- } \\
\text { solved } \\
(\mathbf{m g} / \mathbf{L})\end{array}$ & $\begin{array}{c}\text { Alka- } \\
\text { linity, } \\
\text { lab } \\
(\mathrm{mg} / \mathrm{L} \text { as } \\
\left.\mathrm{CaCO}_{3}\right)\end{array}$ & $\begin{array}{c}\text { Sulfate, } \\
\text { dis- } \\
\text { solved } \\
(\mathrm{mg} \mathrm{as} \\
\left.\mathrm{SO}_{4}\right)\end{array}$ & $\begin{array}{c}\text { Chlo- } \\
\text { ride, } \\
\text { dis- } \\
\text { solved } \\
(\mathrm{mg} / \mathrm{L})\end{array}$ & $\begin{array}{c}\text { Fluo- } \\
\text { ride, } \\
\text { dis- } \\
\text { solved } \\
(\mathrm{mg} / \mathrm{L})\end{array}$ & $\begin{array}{c}\text { Silica, } \\
\text { dis- } \\
\text { solved } \\
(\mathrm{mg} / \mathrm{L} \text { as } \\
\left.\mathrm{SiO}_{2}\right)\end{array}$ & $\begin{array}{c}\text { Nitrate, } \\
\text { dis- } \\
\text { solved } \\
(\mathrm{mg} / \mathrm{L} \text { as } \\
\left.\mathrm{NO}_{3}\right)\end{array}$ \\
\hline $7 / 26 / 2001 \quad 9: 00$ & & 17.3 & 250 & 8.22 & 13.04 & 9.22 & 27.6 & 6.61 & 15.7 & 2.42 & 92.4 & 43.0 & 3.31 & 0.32 & 21.6 & 0.75 \\
\hline 7/26/2001 10:00 & & 17.7 & 249 & 8.27 & 13.85 & 9.30 & 27.3 & 6.41 & 15.6 & 2.42 & -- & -- & -- & -- & 21.3 & -- \\
\hline 7/26/2001 10:01 & $\mathrm{R}$ & -- & -- & -- & -- & -- & 27.1 & 6.60 & 16.1 & 2.45 & 92.1 & 43.0 & 3.33 & 0.34 & 21.9 & 0.59 \\
\hline 7/26/2001 11:00 & & 17.7 & 249 & 8.34 & 15.12 & 9.19 & 27.5 & 6.37 & 15.6 & 2.42 & 91.6 & 42.6 & 3.37 & 0.34 & 21.5 & 0.72 \\
\hline 7/26/2001 12:00 & & 17.7 & 250 & 8.39 & 16.76 & 8.96 & 27.3 & 6.25 & 15.6 & 2.40 & 91.1 & 43.4 & 3.39 & 0.34 & 21.4 & 0.66 \\
\hline $7 / 26 / 2001 \quad 12: 20$ & B & -- & -- & -- & -- & -- & $<0.05$ & $<0.06$ & $<0.04$ & $<0.002$ & $<1$ & $<1$ & $<0.5$ & $<0.2$ & $<0.01$ & $<0.5$ \\
\hline 7/26/2001 13:00 & & 17.7 & 250 & 8.43 & 18.63 & 8.71 & 26.9 & 6.20 & 15.6 & 2.40 & 91.0 & 42.5 & 3.33 & 0.39 & 21.0 & 0.52 \\
\hline 7/26/2001 14:00 & & 17.3 & 251 & 8.49 & 20.21 & 7.97 & 26.7 & 6.16 & 15.4 & 2.36 & 90.8 & 43.4 & 3.34 & 0.34 & 21.2 & 0.50 \\
\hline 7/26/2001 15:00 & & 17.0 & 252 & 8.50 & 21.55 & 8.19 & 27.0 & 6.04 & 15.2 & 2.39 & -- & -- & -- & -- & 21.3 & -- \\
\hline $7 / 26 / 2001 \quad 15: 30$ & & 17.3 & 246 & 8.51 & 21.95 & 8.06 & 27.1 & 6.12 & 15.4 & 2.39 & 90.4 & 43.8 & 3.34 & 0.34 & 21.3 & 0.35 \\
\hline 7/26/2001 16:00 & & 16.9 & 253 & 8.51 & 22.07 & 7.91 & 27.1 & 6.12 & 15.4 & 2.41 & 89.9 & 43.6 & 3.42 & 0.40 & 21.2 & 0.35 \\
\hline $7 / 26 / 2001 \quad 16: 30$ & & 17.3 & 252 & 8.50 & 21.88 & 7.74 & 27.2 & 6.14 & 15.1 & 2.40 & 89.5 & 43.5 & 3.38 & 0.41 & 21.3 & 0.38 \\
\hline 7/26/2001 17:00 & & 17.3 & 252 & 8.49 & 22.00 & 7.66 & 26.9 & 6.03 & 15.4 & 2.39 & 89.8 & 43.6 & 3.33 & 0.35 & 21.3 & 0.47 \\
\hline $7 / 26 / 2001 \quad 17: 30$ & & 16.9 & 252 & 8.47 & 22.00 & 7.46 & 27.0 & 6.02 & 15.4 & 2.40 & 89.6 & 43.9 & 3.53 & 0.40 & 21.4 & 0.35 \\
\hline 7/26/2001 18:00 & & 16.9 & 251 & 8.44 & 22.02 & 7.12 & 26.7 & 6.08 & 15.4 & 2.41 & 89.5 & 44.1 & 3.26 & 0.39 & 21.1 & 0.31 \\
\hline 7/26/2001 18:30 & & 16.9 & 252 & 8.42 & 21.79 & 7.44 & 26.6 & 6.02 & 15.2 & 2.40 & 89.7 & 43.3 & 3.54 & 0.34 & 21.1 & 0.35 \\
\hline 7/26/2001 19:00 & & 16.9 & 252 & 8.40 & 21.72 & 7.40 & 27.0 & 6.04 & 15.4 & 2.43 & 89.5 & 43.6 & 3.38 & 0.40 & 21.1 & 0.35 \\
\hline 7/26/2001 19:30 & & 16.5 & 252 & 8.38 & 21.51 & 7.35 & 27.0 & 6.02 & 15.4 & 2.44 & 90.0 & 44.0 & 3.37 & 0.33 & 21.0 & 0.22 \\
\hline 7/26/2001 20:00 & & 16.1 & 252 & 8.33 & 21.27 & 7.28 & 27.0 & 6.08 & 15.3 & 2.45 & 90.1 & 44.4 & 3.41 & 0.34 & 21.1 & 0.03 \\
\hline 7/26/2001 21:00 & & 16.1 & 253 & 8.17 & 20.49 & 7.24 & 27.4 & 6.31 & 15.7 & 2.49 & 90.1 & 44.3 & 3.41 & 0.34 & 21.5 & 0.41 \\
\hline $7 / 26 / 200122: 00$ & & 16.1 & 254 & 8.08 & 19.73 & 7.19 & 27.7 & 6.25 & 15.4 & 2.48 & 90.6 & 43.4 & 3.66 & 0.30 & 21.6 & 0.47 \\
\hline 7/26/2001 23:00 & & 16.1 & 255 & 8.02 & 18.92 & 7.41 & 27.0 & 6.26 & 15.5 & 2.43 & 91.5 & 43.9 & 3.42 & 0.32 & 21.0 & 0.50 \\
\hline $7 / 27 / 2001 \quad 0: 00$ & & 16.1 & 256 & 8.01 & 18.20 & 7.44 & 27.7 & 6.38 & 15.8 & 2.45 & 92.1 & 43.6 & 3.43 & 0.33 & 21.0 & 0.57 \\
\hline $7 / 27 / 2001 \quad 1: 00$ & & 16.5 & 256 & 8.04 & 17.50 & 7.68 & 27.9 & 6.43 & 15.7 & 2.50 & 93.1 & 44.4 & 3.62 & 0.38 & 21.1 & 0.46 \\
\hline $7 / 27 / 2001 \quad 2: 00$ & & 16.5 & 257 & 8.06 & 16.73 & 7.77 & 28.1 & 6.49 & 16.2 & 2.52 & 93.3 & 44.0 & 3.49 & 0.38 & 21.3 & 0.60 \\
\hline $7 / 27 / 2001 \quad 3: 00$ & & 16.5 & 255 & 8.06 & 15.99 & 7.91 & 28.2 & 6.49 & 16.2 & 2.52 & -- & -- & -- & -- & 21.4 & -- \\
\hline
\end{tabular}


Table 6. Water-quality data for diel investigations in Prickly Pear Creek at site PP-5, Montana, July 25-27, 2001 (Continued).

\begin{tabular}{|c|c|c|c|c|c|c|c|c|c|c|c|c|c|c|c|c|}
\hline Date and time & $\begin{array}{c}\text { QA } \\
\text { sample } \\
\text { type }\end{array}$ & $\begin{array}{c}\text { Alum- } \\
\text { inum, } \\
\text { total } \\
\text { recov- } \\
\text { erable } \\
(\mu \mathrm{g} / \mathrm{L})\end{array}$ & $\begin{array}{c}\text { Alum- } \\
\text { inum, } \\
\text { dis- } \\
\text { solved } \\
(\mu \mathrm{g} / \mathrm{L})\end{array}$ & $\begin{array}{c}\text { Arsenic, } \\
\text { total } \\
\text { recov- } \\
\text { erable } \\
(\mu \mathrm{g} / \mathrm{L})\end{array}$ & $\begin{array}{c}\text { Arsenic, } \\
\text { total, } \\
\text { dis- } \\
\text { solved } \\
(\mu \mathrm{g} / \mathrm{L})\end{array}$ & $\begin{array}{c}\text { Arsenic, } \\
\mathbf{A s}^{+3}, \text { dis- } \\
\text { solved } \\
(\mu \mathrm{g} / \mathrm{L})\end{array}$ & $\begin{array}{c}\text { Barium, } \\
\text { dis- } \\
\text { solved } \\
(\mu \mathrm{g} / \mathrm{L})\end{array}$ & $\begin{array}{c}\text { Beryl- } \\
\text { lium, } \\
\text { dis- } \\
\text { solved } \\
(\mu \mathrm{g} / \mathrm{L})\end{array}$ & $\begin{array}{c}\text { Boron, } \\
\text { dis- } \\
\text { solved } \\
(\mu \mathrm{g} / \mathrm{L})\end{array}$ & $\begin{array}{c}\text { Cad- } \\
\text { mium, } \\
\text { total } \\
\text { recov- } \\
\text { erable } \\
(\mu \mathrm{g} / \mathrm{L}) \\
\end{array}$ & $\begin{array}{c}\text { Cad- } \\
\text { mium, } \\
\text { dis- } \\
\text { solved } \\
(\mu \mathrm{g} / \mathrm{L})\end{array}$ & $\begin{array}{c}\text { Chro- } \\
\text { mium, } \\
\text { dis- } \\
\text { solved } \\
(\mu \mathrm{g} / \mathrm{L})\end{array}$ & $\begin{array}{c}\text { Cobalt, } \\
\text { dis- } \\
\text { solved } \\
(\mu \mathrm{g} / \mathrm{L})\end{array}$ & $\begin{array}{c}\text { Copper, } \\
\text { total } \\
\text { recov- } \\
\text { erable } \\
(\mu \mathrm{g} / \mathrm{L})\end{array}$ & $\begin{array}{c}\text { Copper, } \\
\text { dis- } \\
\text { solved } \\
(\mu \mathrm{g} / \mathrm{L})\end{array}$ & $\begin{array}{c}\text { Iron, } \\
\text { total } \\
\text { recov- } \\
\text { erable } \\
(\mu \mathrm{g} / \mathrm{L})\end{array}$ \\
\hline $7 / 26 / 2001 \quad 9: 00$ & & $<80$ & $<80$ & $<50$ & 4.37 & 0.60 & 27.4 & $<0.1$ & 21.3 & $<1$ & $<1$ & $<1$ & 1.2 & 2.30 & 2.22 & 106 \\
\hline $7 / 26 / 2001$ 10:00 & & $<80$ & $<80$ & $<50$ & 4.32 & 2.01 & 26.4 & $<0.1$ & 21.7 & $<1$ & $<1$ & $<1$ & $<1$ & 2.40 & 2.12 & 119 \\
\hline 7/26/2001 10:01 & $\mathrm{R}$ & -- & $<80$ & -- & 4.40 & $<0.5$ & 25.6 & $<0.1$ & 21.5 & -- & $<1$ & $<1$ & $<1$ & -- & 2.04 & -- \\
\hline 7/26/2001 11:00 & & $<80$ & $<80$ & $<50$ & 4.58 & 0.66 & 26.4 & $<0.1$ & 21.3 & $<1$ & $<1$ & $<1$ & $<1$ & 2.41 & 2.19 & 114 \\
\hline 7/26/2001 12:00 & & $<80$ & $<80$ & $<50$ & 4.77 & 0.72 & 25.2 & $<0.1$ & 21.1 & $<1$ & $<1$ & $<1$ & $<1$ & 2.53 & 1.93 & 113 \\
\hline $7 / 26 / 2001 \quad 12: 20$ & B & $<80$ & $<80$ & $<50$ & $<0.1$ & $<0.5$ & $<0.5$ & $<0.1$ & $<3$ & $<1$ & $<1$ & $<1$ & $<1$ & 1.23 & 0.63 & $<9$ \\
\hline 7/26/2001 13:00 & & $<80$ & $<80$ & $<50$ & 4.83 & 0.80 & 24.5 & $<0.1$ & 20.6 & $<1$ & $<1$ & $<1$ & $<1$ & 2.48 & 2.22 & 121 \\
\hline 7/26/2001 14:00 & & $<80$ & $<80$ & $<50$ & 4.85 & 0.52 & 24.5 & $<0.1$ & 20.9 & $<1$ & $<1$ & $<1$ & $<1$ & 2.83 & 2.04 & 118 \\
\hline 7/26/2001 15:00 & & $<80$ & $<80$ & $<50$ & 4.93 & 1.00 & 24.2 & $<0.1$ & 20.8 & $<1$ & $<1$ & $<1$ & $<1$ & 3.08 & 2.21 & 117 \\
\hline $7 / 26 / 2001 \quad 15: 30$ & & $<80$ & $<80$ & $<50$ & 5.38 & $<0.5$ & 24.2 & $<0.1$ & 20.9 & $<1$ & $<1$ & $<1$ & $<1$ & 2.69 & 2.23 & 118 \\
\hline $7 / 26 / 2001$ 16:00 & & $<80$ & $<80$ & $<50$ & 5.49 & $<0.5$ & 24.4 & $<0.1$ & 20.7 & $<1$ & $<1$ & $<1$ & $<1$ & 2.88 & 2.22 & 128 \\
\hline $7 / 26 / 200116: 30$ & & $<80$ & $<80$ & $<50$ & 5.46 & 1.34 & 23.9 & $<0.1$ & 21.1 & $<1$ & $<1$ & $<1$ & $<1$ & 2.77 & 2.15 & 147 \\
\hline 7/26/2001 17:00 & & $<80$ & $<80$ & $<50$ & 5.22 & $<0.5$ & 24.0 & $<0.1$ & 20.5 & $<1$ & $<1$ & $<1$ & $<1$ & 2.49 & 2.66 & 118 \\
\hline $7 / 26 / 2001 \quad 17: 30$ & & $<80$ & $<80$ & $<50$ & 5.58 & 0.62 & 24.7 & $<0.1$ & 20.6 & $<1$ & $<1$ & $<1$ & $<1$ & 3.06 & 2.25 & 146 \\
\hline 7/26/2001 18:00 & & $<80$ & $<80$ & $<50$ & 5.70 & $<0.5$ & 24.5 & $<0.1$ & 20.9 & $<1$ & $<1$ & $<1$ & $<1$ & 2.96 & 2.12 & 127 \\
\hline $7 / 26 / 200118: 30$ & & $<80$ & $<80$ & $<50$ & 5.62 & $<0.5$ & 24.3 & $<0.1$ & 20.6 & $<1$ & $<1$ & $<1$ & $<1$ & 3.01 & 2.18 & 137 \\
\hline 7/26/2001 19:00 & & $<80$ & $<80$ & $<50$ & 5.62 & 1.15 & 24.8 & $<0.1$ & 20.7 & $<1$ & $<1$ & $<1$ & $<1$ & 2.93 & 2.25 & 128 \\
\hline $7 / 26 / 200119: 30$ & & $<80$ & $<80$ & $<50$ & 5.78 & 0.87 & 24.8 & $<0.1$ & 20.3 & $<1$ & $<1$ & $<1$ & $<1$ & 2.78 & 2.15 & 126 \\
\hline $7 / 26 / 200120: 00$ & & $<80$ & $<80$ & $<50$ & 5.70 & 0.68 & 24.6 & $<0.1$ & 20.9 & $<1$ & $<1$ & $<1$ & $<1$ & 2.62 & 2.14 & 117 \\
\hline 7/26/2001 21:00 & & $<80$ & $<80$ & $<50$ & 5.47 & 0.56 & 25.6 & $<0.1$ & 21.8 & $<1$ & $<1$ & $<1$ & $<1$ & 3.12 & 2.27 & 120 \\
\hline 7/26/2001 22:00 & & $<80$ & $<80$ & $<50$ & 5.44 & 1.24 & 25.8 & $<0.1$ & 21.9 & $<1$ & $<1$ & $<1$ & $<1$ & 3.50 & 2.59 & 143 \\
\hline 7/26/2001 23:00 & & 83.4 & $<80$ & $<50$ & 5.32 & 0.73 & 26.5 & $<0.1$ & 22.1 & $<1$ & $<1$ & $<1$ & $<1$ & 3.29 & 2.35 & 163 \\
\hline $7 / 27 / 2001 \quad 0: 00$ & & $<80$ & $<80$ & $<50$ & 5.24 & 1.07 & 27.0 & $<0.1$ & 22.2 & $<1$ & $<1$ & $<1$ & $<1$ & 2.92 & 2.25 & 113 \\
\hline $7 / 27 / 2001 \quad 1: 00$ & & $<80$ & $<80$ & $<50$ & 5.18 & $<0.5$ & 27.5 & $<0.1$ & 22.0 & $<1$ & $<1$ & $<1$ & $<1$ & 3.40 & 2.12 & 164 \\
\hline $7 / 27 / 2001 \quad 2: 00$ & & $<80$ & $<80$ & $<50$ & 5.03 & 0.78 & 27.8 & $<0.1$ & 21.9 & $<1$ & $<1$ & $<1$ & $<1$ & 3.38 & 2.25 & 154 \\
\hline $7 / 27 / 2001 \quad 3: 00$ & & -- & $<80$ & -- & 4.92 & 1.02 & 27.5 & $<0.1$ & 22.4 & -- & $<1$ & $<1$ & $<1$ & -- & 2.36 & -- \\
\hline
\end{tabular}


Table 6. Water-quality data for diel investigations in Prickly Pear Creek at site PP-5, Montana, July 25-27, 2001 (Continued).

\begin{tabular}{|c|c|c|c|c|c|c|c|c|c|c|c|c|c|c|c|c|c|c|}
\hline Date and time & $\begin{array}{c}\text { QA } \\
\text { sample } \\
\text { type }\end{array}$ & $\begin{array}{l}\text { Iron, } \\
\text { total, } \\
\text { dis- } \\
\text { solved } \\
(\mu \mathrm{g} / \mathrm{L})\end{array}$ & $\begin{array}{c}\text { Iron, } \\
\text { Fe }^{+2} \\
\text { dis- } \\
\text { solved } \\
(\mu \mathrm{g} / \mathrm{L})\end{array}$ & $\begin{array}{l}\text { Lead, } \\
\text { total } \\
\text { recov- } \\
\text { erable } \\
(\mu \mathrm{g} / \mathrm{L})\end{array}$ & $\begin{array}{c}\text { Lead, } \\
\text { dis- } \\
\text { solved } \\
(\mu \mathrm{g} / \mathrm{L})\end{array}$ & $\begin{array}{l}\text { Lith- } \\
\text { ium, } \\
\text { dis- } \\
\text { solved } \\
(\mu \mathrm{g} / \mathrm{L})\end{array}$ & $\begin{array}{c}\text { Manga- } \\
\text { nese, } \\
\text { total } \\
\text { recov- } \\
\text { erable } \\
(\mu \mathrm{g} / \mathrm{L}) \\
\end{array}$ & $\begin{array}{l}\text { Manga- } \\
\text { nese, } \\
\text { dis- } \\
\text { solved } \\
(\mu \mathrm{g} / \mathrm{L})\end{array}$ & $\begin{array}{c}\text { Nickel, } \\
\text { total } \\
\text { recov- } \\
\text { erable } \\
(\mu \mathrm{g} / \mathrm{L})\end{array}$ & $\begin{array}{c}\text { Nickel, } \\
\text { dis- } \\
\text { solved } \\
(\mu \mathrm{g} / \mathrm{L})\end{array}$ & 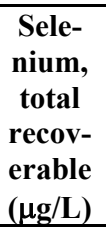 & $\begin{array}{c}\text { Sele- } \\
\text { nium, } \\
\text { dis- } \\
\text { solved } \\
(\mu \mathrm{g} / \mathrm{L})\end{array}$ & $\begin{array}{c}\text { Stron- } \\
\text { tium, } \\
\text { total } \\
\text { recov- } \\
\text { erable } \\
(\mu \mathrm{g} / \mathrm{L}) \\
\end{array}$ & $\begin{array}{c}\text { Stron- } \\
\text { tium, } \\
\text { dis- } \\
\text { solved } \\
(\mu \mathrm{g} / \mathrm{L})\end{array}$ & $\begin{array}{l}\text { Vana- } \\
\text { dium, } \\
\text { total } \\
\text { recov- } \\
\text { erable } \\
(\mu \mathrm{g} / \mathrm{L}) \\
\end{array}$ & $\begin{array}{c}\text { Vana- } \\
\text { dium, } \\
\text { dis- } \\
\text { solved } \\
(\mu \mathrm{g} / \mathrm{L})\end{array}$ & $\begin{array}{l}\text { Zinc, } \\
\text { total } \\
\text { recov- } \\
\text { erable } \\
(\mu \mathrm{g} / \mathrm{L})\end{array}$ & $\begin{array}{c}\text { Zinc, } \\
\text { dis- } \\
\text { solved } \\
(\mu \mathrm{g} / \mathrm{L})\end{array}$ \\
\hline $7 / 26 / 2001 \quad 9: 00$ & & 27.5 & 27.7 & $<8$ & $<8$ & 22.6 & 40.2 & 30.4 & $<2$ & $<2$ & $<50$ & $<50$ & 250 & 252 & $<1$ & $<1$ & 68.1 & 49.4 \\
\hline 7/26/2001 10:00 & & 28.2 & -- & $<8$ & $<8$ & 22.0 & 42.7 & 29.9 & $<2$ & $<2$ & $<50$ & $<50$ & 249 & 248 & $<1$ & $<1$ & 64.7 & 50.0 \\
\hline 7/26/2001 10:01 & $\mathrm{R}$ & 27.0 & 24.6 & -- & $<8$ & 22.6 & -- & 25.4 & -- & $<2$ & -- & $<50$ & -- & 250 & -- & $<1$ & -- & -- \\
\hline 7/26/2001 11:00 & & 27.3 & 25.9 & $<8$ & $<8$ & 21.7 & 40.8 & 28.4 & 2.4 & $<2$ & $<50$ & $<50$ & 252 & 249 & $<1$ & $<1$ & 69.6 & 44.7 \\
\hline $7 / 26 / 2001 \quad 12: 00$ & & 24.0 & 23.3 & $<8$ & $<8$ & 21.8 & 39.0 & 27.2 & $<2$ & $<2$ & $<50$ & $<50$ & 251 & 249 & $<1$ & $<1$ & 54.7 & 39.9 \\
\hline $7 / 26 / 2001 \quad 12: 20$ & B & $<2$ & $<2$ & $<8$ & $<8$ & $<8$ & $<1$ & $<1$ & $<2$ & $<2$ & $<50$ & $<50$ & $<1$ & $<1$ & $<1$ & $<1$ & $<5$ & $<5$ \\
\hline 7/26/2001 13:00 & & 20.8 & 19.6 & $<8$ & $<8$ & 21.3 & 38.3 & 25.3 & $<2$ & $<2$ & $<50$ & $<50$ & 250 & 245 & $<1$ & $<1$ & 52.3 & 33.5 \\
\hline $7 / 26 / 2001$ 14:00 & & 21.3 & 19.9 & $<8$ & $<8$ & 21.6 & 36.9 & 23.4 & $<2$ & $<2$ & $<50$ & $<50$ & 249 & 244 & $<1$ & $<1$ & 46.5 & 30.4 \\
\hline 7/26/2001 15:00 & & 15.7 & 14.8 & $<8$ & $<8$ & 21.7 & 35.4 & 22.1 & $<2$ & $<2$ & $<50$ & $<50$ & 247 & 242 & $<1$ & $<1$ & 43.2 & 24.3 \\
\hline $7 / 26 / 2001 \quad 15: 30$ & & 18.1 & 18.3 & $<8$ & $<8$ & 21.8 & 35.4 & 22.0 & $<2$ & $<2$ & $<50$ & $<50$ & 248 & 244 & $<1$ & $<1$ & 41.4 & 22.4 \\
\hline 7/26/2001 16:00 & & 18.4 & 17.9 & $<8$ & $<8$ & 22.0 & 38.3 & 22.4 & $<2$ & $<2$ & $<50$ & $<50$ & 250 & 245 & 1.1 & 1.0 & 42.2 & 22.9 \\
\hline $7 / 26 / 200116: 30$ & & 19.1 & 16.7 & $<8$ & $<8$ & 21.5 & 42.6 & 24.4 & $<2$ & $<2$ & $<50$ & $<50$ & 252 & 244 & 1.1 & 1.1 & 44.5 & 21.5 \\
\hline 7/26/2001 17:00 & & 16.7 & 16.2 & $<8$ & $<8$ & 21.3 & 35.5 & 22.0 & $<2$ & $<2$ & $<50$ & $<50$ & 253 & 243 & 1.1 & 1.0 & 42.4 & 24.0 \\
\hline $7 / 26 / 2001 \quad 17: 30$ & & 18.1 & 16.6 & $<8$ & $<8$ & 21.7 & 40.9 & 24.3 & $<2$ & $<2$ & $<50$ & $<50$ & 251 & 244 & 1.2 & $<1$ & 45.1 & 22.4 \\
\hline 7/26/2001 18:00 & & 15.7 & 15.2 & $<8$ & $<8$ & 21.4 & 36.8 & 21.5 & $<2$ & $<2$ & $<50$ & $<50$ & 253 & 243 & 1.3 & $<1$ & 44.0 & 20.6 \\
\hline $7 / 26 / 200118: 30$ & & 16.2 & 15.2 & $<8$ & $<8$ & 21.4 & 40.4 & 21.7 & $<2$ & $<2$ & $<50$ & $<50$ & 252 & 241 & 1.2 & 1.2 & 43.7 & 21.5 \\
\hline $7 / 26 / 2001$ 19:00 & & 18.0 & 17.4 & $<8$ & $<8$ & 21.7 & 37.3 & 21.8 & $<2$ & $<2$ & $<50$ & $<50$ & 253 & 245 & $<1$ & $<1$ & 42.2 & 22.8 \\
\hline $7 / 26 / 200119: 30$ & & 18.7 & 17.9 & $<8$ & $<8$ & 21.7 & 38.1 & 22.4 & $<2$ & $<2$ & $<50$ & $<50$ & 250 & 245 & 1.2 & 1.2 & 41.6 & 22.7 \\
\hline 7/26/2001 20:00 & & 18.1 & 16.0 & $<8$ & $<8$ & 21.7 & 35.8 & 21.8 & $<2$ & $<2$ & $<50$ & $<50$ & 251 & 247 & 1.3 & $<1$ & 45.4 & 25.2 \\
\hline 7/26/2001 21:00 & & 20.9 & 19.4 & $<8$ & $<8$ & 21.7 & 37.2 & 23.3 & $<2$ & $<2$ & $<50$ & $<50$ & 252 & 248 & 1.3 & 1.1 & 47.2 & 30.6 \\
\hline $7 / 26 / 200122: 00$ & & 19.8 & 19.6 & $<8$ & $<8$ & 21.2 & 41.9 & 25.9 & $<2$ & $<2$ & $<50$ & $<50$ & 255 & 250 & 1.2 & $<1$ & 55.1 & 35.6 \\
\hline 7/26/2001 23:00 & & 23.5 & 23.0 & $<8$ & $<8$ & 21.7 & 44.8 & 29.1 & $<2$ & $<2$ & $<50$ & $<50$ & 255 & 249 & 1.4 & $<1$ & 61.5 & 38.7 \\
\hline $7 / 27 / 2001 \quad 0: 00$ & & 23.8 & 24.7 & $<8$ & $<8$ & 21.8 & 39.8 & 29.0 & $<2$ & $<2$ & $<50$ & $<50$ & 256 & 252 & 1.4 & $<1$ & 60.2 & 42.7 \\
\hline $7 / 27 / 2001 \quad 1: 00$ & & 28.6 & 28.6 & $<8$ & $<8$ & 21.8 & 51.4 & 35.3 & $<2$ & $<2$ & $<50$ & $<50$ & 260 & 253 & 1.4 & $<1$ & 69.0 & 45.4 \\
\hline $7 / 27 / 2001 \quad 2: 00$ & & 28.5 & 25.0 & $<8$ & $<8$ & 22.5 & 49.3 & 30.7 & $<2$ & $<2$ & $<50$ & $<50$ & 261 & 256 & 1.0 & $<1$ & 80.6 & 49.1 \\
\hline $7 / 27 / 2001 \quad 3: 00$ & & 28.8 & 26.7 & -- & $<8$ & 22.8 & -- & 29.7 & -- & $<2$ & -- & $<50$ & -- & 253 & -- & $<1$ & -- & 51.2 \\
\hline
\end{tabular}


Table 6. Water-quality data for diel investigations in Prickly Pear Creek at site PP-5, Montana, July 25-27, 2001 (Continued).

\begin{tabular}{|c|c|c|c|c|c|c|c|c|c|c|c|c|c|c|c|c|}
\hline Date and time & $\begin{array}{c}\text { QA } \\
\text { sample } \\
\text { type }\end{array}$ & $\begin{array}{l}\text { Stream- } \\
\text { flow, } \\
\text { instan- } \\
\text { taneous } \\
\left(\mathbf{f t}^{3} / \mathbf{s}\right)\end{array}$ & 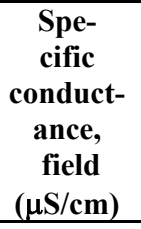 & $\begin{array}{c}\text { pH, } \\
\text { field } \\
\text { (su) }\end{array}$ & $\begin{array}{l}\text { Tem- } \\
\text { pera- } \\
\text { ture, } \\
\text { water } \\
\left({ }^{\circ} \mathrm{C}\right)\end{array}$ & $\begin{array}{c}\text { Dis- } \\
\text { solved } \\
\text { oxygen, } \\
\text { field } \\
(\mathrm{mg} / \mathrm{L})\end{array}$ & $\begin{array}{c}\text { Cal- } \\
\text { cium, } \\
\text { dis- } \\
\text { solved } \\
(\mathbf{m g} / \mathbf{L})\end{array}$ & $\begin{array}{l}\text { Magne- } \\
\text { sium, } \\
\text { dissolved } \\
(\mathrm{mg} / \mathrm{L})\end{array}$ & $\begin{array}{c}\text { So- } \\
\text { dium, } \\
\text { dis- } \\
\text { solved } \\
(\mathrm{mg} / \mathrm{L})\end{array}$ & $\begin{array}{c}\text { Potas- } \\
\text { sium, } \\
\text { dis- } \\
\text { solved } \\
(\mathrm{mg} / \mathrm{L})\end{array}$ & $\begin{array}{c}\text { Alka- } \\
\text { linity, } \\
\text { lab } \\
(\mathrm{mg} / \mathrm{L} \text { as } \\
\left.\mathrm{CaCO}_{3}\right)\end{array}$ & $\begin{array}{c}\begin{array}{c}\text { Sulfate, } \\
\text { dis- } \\
\text { solved } \\
(\mathrm{mg} \text { as }\end{array} \\
\left.\mathrm{SO}_{4}\right)\end{array}$ & $\begin{array}{c}\text { Chlo- } \\
\text { ride, } \\
\text { dis- } \\
\text { solved } \\
(\mathrm{mg} / \mathrm{L})\end{array}$ & $\begin{array}{l}\text { Fluo- } \\
\text { ride, } \\
\text { dis- } \\
\text { solved } \\
(\mathrm{mg} / \mathrm{L})\end{array}$ & $\begin{array}{c}\text { Silica, } \\
\text { dis- } \\
\text { solved } \\
(\mathrm{mg} / \mathrm{L} \text { as } \\
\left.\mathrm{SiO}_{2}\right) \\
\end{array}$ & $\begin{array}{c}\text { Nitrate, } \\
\text { dis- } \\
\text { solved } \\
(\mathrm{mg} / \mathrm{L} \text { as } \\
\left.\mathrm{NO}_{3}\right)\end{array}$ \\
\hline $7 / 27 / 2001 \quad 4: 00$ & & 16.9 & 256 & 8.06 & 15.23 & 8.10 & -- & -- & -- & -- & 93.8 & 44.0 & 3.40 & 0.39 & -- & 0.62 \\
\hline $7 / 27 / 2001 \quad 5: 00$ & & 16.5 & 255 & 8.05 & 14.51 & 8.30 & -- & -- & -- & -- & 94.3 & 43.7 & 3.45 & 0.32 & -- & 0.70 \\
\hline $7 / 27 / 2001 \quad 5: 30$ & & 16.9 & 254 & 8.04 & 14.14 & 8.34 & -- & -- & -- & -- & 93.9 & 43.4 & 3.48 & 0.33 & -- & 0.60 \\
\hline $7 / 27 / 2001 \quad 6: 00$ & & 16.5 & 255 & 8.04 & 13.82 & 8.45 & -- & -- & -- & -- & 94.3 & 43.6 & 3.54 & 0.39 & -- & 0.60 \\
\hline $7 / 27 / 2001 \quad 6: 30$ & & 16.5 & 254 & 8.05 & 13.47 & 8.59 & -- & -- & -- & -- & 94.0 & 44.3 & 3.48 & 0.37 & -- & 0.65 \\
\hline $7 / 27 / 2001 \quad 7: 00$ & & 16.9 & 254 & 8.07 & 13.15 & 8.69 & -- & -- & -- & -- & 93.9 & 43.8 & 3.46 & 0.37 & -- & 0.66 \\
\hline $7 / 27 / 2001 \quad 7: 30$ & & 16.9 & 253 & 8.08 & 12.87 & 8.81 & -- & -- & -- & -- & 93.6 & 43.1 & 3.38 & 0.36 & -- & 0.62 \\
\hline $7 / 27 / 2001 \quad 8: 00$ & & 16.9 & 250 & 8.08 & 12.65 & 8.97 & -- & -- & -- & -- & 93.5 & 42.7 & 3.50 & 0.32 & -- & 0.66 \\
\hline $7 / 27 / 2001 \quad 8: 30$ & & 17.3 & 250 & 8.11 & 12.57 & 9.07 & -- & -- & -- & -- & 93.5 & 42.4 & 3.34 & 0.36 & -- & 0.45 \\
\hline $7 / 27 / 2001 \quad 8: 31$ & $\mathrm{R}$ & -- & -- & -- & -- & -- & -- & -- & -- & -- & 93.2 & 43.7 & 3.47 & 0.34 & -- & 0.53 \\
\hline $7 / 27 / 2001 \quad 9: 00$ & & 17.7 & 248 & 8.11 & 12.76 & 9.28 & -- & -- & -- & -- & 92.7 & 42.5 & 3.41 & 0.34 & -- & 0.53 \\
\hline $7 / 27 / 2001 \quad 9: 30$ & & 17.3 & 250 & 8.16 & 13.17 & 9.23 & 28.0 & 6.56 & 15.6 & 2.39 & 93.0 & 42.9 & 3.61 & 0.34 & 21.3 & 0.53 \\
\hline 7/27/2001 10:00 & & 17.3 & 250 & 8.17 & 13.68 & 9.25 & -- & -- & -- & -- & 93.1 & 43.1 & 3.40 & 0.36 & -- & 0.55 \\
\hline $7 / 27 / 200111: 00$ & & 17.7 & 250 & 8.24 & 15.10 & 9.17 & 27.9 & 6.62 & 15.9 & 2.42 & 92.7 & 42.7 & 3.41 & 0.36 & 21.4 & 0.52 \\
\hline
\end{tabular}


Table 6. Water-quality data for diel investigations in Prickly Pear Creek at site PP-5, Montana, July 25-27, 2001 (Continued)

\begin{tabular}{|c|c|c|c|c|c|c|c|c|c|c|c|c|c|c|c|c|}
\hline Date and time & $\begin{array}{c}\text { QA } \\
\text { sample } \\
\text { type }\end{array}$ & $\begin{array}{c}\text { Alum- } \\
\text { inum, } \\
\text { total } \\
\text { recov- } \\
\text { erable } \\
(\mu \mathrm{g} / \mathrm{L})\end{array}$ & $\begin{array}{c}\text { Alum- } \\
\text { inum, } \\
\text { dis- } \\
\text { solved } \\
(\mu \mathrm{g} / \mathrm{L})\end{array}$ & $\begin{array}{c}\text { Arsenic, } \\
\text { total } \\
\text { recov- } \\
\text { erable } \\
(\mu \mathrm{g} / \mathrm{L})\end{array}$ & $\begin{array}{c}\text { Arsenic, } \\
\text { total, } \\
\text { dis- } \\
\text { solved } \\
(\mu \mathrm{g} / \mathrm{L})\end{array}$ & $\begin{array}{c}\text { Arsenic, } \\
\text { As }^{+3}, \text { dis- } \\
\text { solved } \\
(\mu \mathrm{g} / \mathrm{L})\end{array}$ & $\begin{array}{c}\text { Barium, } \\
\text { dis- } \\
\text { solved } \\
(\mu \mathrm{g} / \mathrm{L})\end{array}$ & $\begin{array}{c}\text { Beryl- } \\
\text { lium, } \\
\text { dis- } \\
\text { solved } \\
(\mu \mathrm{g} / \mathrm{L})\end{array}$ & $\begin{array}{c}\text { Boron, } \\
\text { dis- } \\
\text { solved } \\
(\mu \mathrm{g} / \mathrm{L})\end{array}$ & $\begin{array}{c}\text { Cad- } \\
\text { mium, } \\
\text { total } \\
\text { recov- } \\
\text { erable } \\
(\mu \mathrm{g} / \mathrm{L}) \\
\end{array}$ & $\begin{array}{c}\text { Cad- } \\
\text { mium, } \\
\text { dis- } \\
\text { solved } \\
(\mu \mathrm{g} / \mathrm{L})\end{array}$ & $\begin{array}{c}\text { Chro- } \\
\text { mium, } \\
\text { dis- } \\
\text { solved } \\
(\mu \mathrm{g} / \mathrm{L})\end{array}$ & $\begin{array}{c}\text { Cobalt, } \\
\text { dis- } \\
\text { solved } \\
(\mu \mathrm{g} / \mathrm{L})\end{array}$ & $\begin{array}{c}\text { Copper, } \\
\text { total } \\
\text { recov- } \\
\text { erable } \\
(\mu \mathrm{g} / \mathrm{L})\end{array}$ & $\begin{array}{c}\text { Copper, } \\
\text { dis- } \\
\text { solved } \\
(\mu \mathrm{g} / \mathrm{L})\end{array}$ & $\begin{array}{l}\text { Iron, } \\
\text { total } \\
\text { recov- } \\
\text { erable } \\
(\mu \mathrm{g} / \mathrm{L})\end{array}$ \\
\hline $7 / 27 / 2001 \quad 4: 00$ & & -- & -- & -- & 4.75 & 0.88 & -- & -- & -- & -- & -- & -- & -- & -- & -- & -- \\
\hline $7 / 27 / 2001 \quad 5: 00$ & & -- & -- & -- & 4.58 & 0.65 & -- & -- & -- & -- & -- & -- & -- & -- & -- & -- \\
\hline $7 / 27 / 2001 \quad 5: 30$ & & -- & -- & -- & 4.78 & 0.91 & -- & -- & -- & -- & -- & -- & -- & -- & -- & -- \\
\hline $7 / 27 / 2001 \quad 6: 00$ & & -- & -- & -- & 4.63 & $<0.5$ & -- & -- & -- & -- & -- & -- & -- & -- & -- & -- \\
\hline $7 / 27 / 2001 \quad 6: 30$ & & -- & -- & -- & 4.46 & $<0.5$ & -- & -- & -- & -- & -- & -- & -- & -- & -- & -- \\
\hline $7 / 27 / 2001 \quad 7: 00$ & & -- & -- & -- & 4.44 & 0.95 & -- & -- & -- & -- & -- & -- & -- & -- & -- & -- \\
\hline $7 / 27 / 2001 \quad 7: 30$ & & -- & -- & -- & 4.67 & $<0.5$ & -- & -- & -- & -- & -- & -- & -- & -- & -- & -- \\
\hline $7 / 27 / 2001 \quad 8: 00$ & & -- & -- & -- & 4.54 & $<0.5$ & -- & -- & -- & -- & -- & -- & -- & -- & -- & -- \\
\hline $7 / 27 / 2001 \quad 8: 30$ & & -- & -- & -- & 4.25 & $<0.5$ & -- & -- & -- & -- & -- & -- & -- & -- & -- & -- \\
\hline $7 / 27 / 2001 \quad 8: 31$ & $\mathrm{R}$ & -- & -- & -- & 4.26 & $<0.5$ & -- & -- & -- & -- & -- & -- & -- & -- & -- & -- \\
\hline $7 / 27 / 2001 \quad 9: 00$ & & -- & -- & -- & 4.47 & $<0.5$ & -- & -- & -- & -- & -- & -- & -- & -- & -- & -- \\
\hline $7 / 27 / 2001 \quad 9: 30$ & & $<80$ & $<80$ & $<50$ & 4.36 & 0.75 & 25.1 & $<0.1$ & 21.3 & $<1$ & $<1$ & $<1$ & 1.8 & 2.46 & 2.14 & 116 \\
\hline 7/27/2001 10:00 & & -- & -- & -- & 4.50 & $<0.5$ & -- & -- & -- & -- & -- & -- & -- & -- & -- & -- \\
\hline 7/27/2001 11:00 & & $<80$ & $<80$ & $<50$ & 4.50 & $<0.5$ & 25.2 & $<0.1$ & 21.9 & $<1$ & $<1$ & $<1$ & $<1$ & 2.64 & 2.23 & 111 \\
\hline
\end{tabular}


Table 6. Water-quality data for diel investigations in Prickly Pear Creek at site PP-5, Montana, July 25-27, 2001 (Continued)

\begin{tabular}{|c|c|c|c|c|c|c|c|c|c|c|c|c|c|c|c|c|c|c|}
\hline Date and time & $\begin{array}{c}\text { QA } \\
\text { sample } \\
\text { type }\end{array}$ & $\begin{array}{c}\text { Iron, } \\
\text { total, } \\
\text { dis- } \\
\text { solved } \\
(\mu \mathrm{g} / \mathrm{L})\end{array}$ & $\begin{array}{c}\text { Iron, } \\
\mathrm{Fe}^{+2}, \\
\text { dis- } \\
\text { solved } \\
(\mu \mathrm{g} / \mathrm{L})\end{array}$ & $\begin{array}{c}\text { Lead, } \\
\text { total } \\
\text { recov- } \\
\text { erable } \\
(\mu \mathrm{g} / \mathrm{L})\end{array}$ & $\begin{array}{c}\text { Lead, } \\
\text { dis- } \\
\text { solved } \\
(\mu \mathrm{g} / \mathrm{L})\end{array}$ & $\begin{array}{l}\text { Lith- } \\
\text { ium, } \\
\text { dis- } \\
\text { solved } \\
(\mu \mathrm{g} / L)\end{array}$ & $\begin{array}{c}\text { Manga- } \\
\text { nese, } \\
\text { total } \\
\text { recov- } \\
\text { erable } \\
(\mu \mathrm{g} / \mathrm{L}) \\
\end{array}$ & $\begin{array}{c}\text { Manga- } \\
\text { nese, } \\
\text { dis- } \\
\text { solved } \\
(\mu \mathrm{g} / \mathrm{L})\end{array}$ & $\begin{array}{c}\text { Nickel, } \\
\text { total } \\
\text { recov- } \\
\text { erable } \\
(\mu \mathrm{g} / \mathrm{L})\end{array}$ & $\begin{array}{c}\text { Nickel, } \\
\text { dis- } \\
\text { solved } \\
(\mu \mathrm{g} / \mathrm{L})\end{array}$ & $\begin{array}{c}\text { Sele- } \\
\text { nium, } \\
\text { total } \\
\text { recov- } \\
\text { erable } \\
(\mu \mathrm{g} / \mathrm{L}) \\
\end{array}$ & $\begin{array}{c}\text { Sele- } \\
\text { nium, } \\
\text { dis- } \\
\text { solved } \\
(\mu \mathrm{g} / \mathrm{L})\end{array}$ & $\begin{array}{c}\text { Stron- } \\
\text { tium, } \\
\text { total } \\
\text { recov- } \\
\text { erable } \\
(\mu \mathrm{g} / \mathrm{L}) \\
\end{array}$ & $\begin{array}{c}\text { Stron- } \\
\text { tium, } \\
\text { dis- } \\
\text { solved } \\
(\mu \mathrm{g} / \mathrm{L})\end{array}$ & $\begin{array}{l}\text { Vana- } \\
\text { dium, } \\
\text { total } \\
\text { recov- } \\
\text { erable } \\
(\mu \mathrm{g} / \mathrm{L}) \\
\end{array}$ & $\begin{array}{c}\text { Vana- } \\
\text { dium, } \\
\text { dis- } \\
\text { solved } \\
(\mu \mathrm{g} / \mathrm{L})\end{array}$ & $\begin{array}{l}\text { Zinc, } \\
\text { total } \\
\text { recov- } \\
\text { erable } \\
(\mu \mathrm{g} / \mathrm{L})\end{array}$ & $\begin{array}{c}\text { Zinc, } \\
\text { dis- } \\
\text { solved } \\
(\mu \mathrm{g} / \mathrm{L})\end{array}$ \\
\hline $7 / 27 / 2001 \quad 4: 00$ & & 28.8 & 27.0 & -- & -- & -- & -- & -- & -- & -- & -- & -- & -- & - & -- & -- & -- & -- \\
\hline $7 / 27 / 2001 \quad 5: 00$ & & 29.4 & 26.5 & -- & -- & -- & -- & -- & -- & -- & -- & -- & -- & -- & -- & -- & -- & -- \\
\hline $7 / 27 / 2001 \quad 5: 30$ & & 30.6 & 28.7 & -- & -- & -- & -- & -- & -- & -- & -- & -- & -- & -- & -- & -- & -- & -- \\
\hline $7 / 27 / 2001 \quad 6: 00$ & & 30.0 & 28.4 & -- & -- & -- & -- & -- & -- & -- & -- & -- & -- & -- & -- & -- & -- & -- \\
\hline $7 / 27 / 2001 \quad 6: 30$ & & 29.9 & 29.4 & -- & -- & -- & -- & -- & -- & -- & -- & -- & -- & -- & -- & -- & -- & -- \\
\hline $7 / 27 / 2001 \quad 7: 00$ & & 30.1 & 28.1 & -- & -- & -- & -- & -- & -- & -- & -- & -- & -- & -- & -- & -- & -- & -- \\
\hline $7 / 27 / 2001 \quad 7: 30$ & & 30.3 & 28.4 & -- & -- & -- & -- & -- & -- & -- & -- & -- & -- & -- & -- & -- & -- & -- \\
\hline $7 / 27 / 2001 \quad 8: 00$ & & 29.3 & 27.7 & -- & -- & -- & -- & -- & -- & -- & -- & -- & -- & -- & -- & -- & -- & -- \\
\hline $7 / 27 / 2001 \quad 8: 30$ & & 29.3 & 29.0 & -- & -- & -- & -- & -- & -- & -- & -- & -- & -- & -- & -- & -- & -- & -- \\
\hline $7 / 27 / 2001 \quad 8: 31$ & $\mathrm{R}$ & 30.5 & 27.4 & -- & -- & -- & -- & -- & -- & -- & -- & -- & -- & -- & -- & -- & -- & -- \\
\hline $7 / 27 / 2001 \quad 9: 00$ & & 29.0 & 27.8 & -- & -- & -- & -- & -- & -- & -- & -- & -- & -- & -- & -- & -- & -- & -- \\
\hline $7 / 27 / 2001 \quad 9: 30$ & & 24.8 & 21.8 & $<8$ & $<8$ & 22.3 & 41.7 & 28.9 & $<2$ & $<2$ & $<50$ & $<50$ & 253 & 245 & 1.2 & $<1$ & 67.8 & 67.4 \\
\hline 7/27/2001 10:00 & & 26.6 & 23.7 & -- & -- & -- & -- & -- & -- & -- & -- & -- & -- & -- & -- & -- & -- & -- \\
\hline 7/27/2001 11:00 & & 24.0 & 21.2 & $<8$ & $<8$ & 22.1 & 40.0 & 28.1 & $<2$ & $<2$ & $<50$ & $<50$ & 252 & 247 & 1.2 & $<1$ & 63.5 & 62.2 \\
\hline
\end{tabular}


Table 7. Water-quality data for diel investigations in Middle Fork Warm Springs Creek at site WS-3, Montana, June 26-27, 2001.

[Samples analyzed by the U.S. Geological Survey National Water Quality Laboratory, Denver, Colo. Analytical data are reported to a maximum of three significant figures to provide sufficient resolution for detecting small differences between samples closely spaced in time or distance. Similarly, field measurements from electronic recorders have not been rounded to illustrate the pattern of relative change between measurements. Abbreviations: $\mathrm{ft}^{3} / \mathrm{s}$, cubic feet per second; ${ }^{\circ} \mathrm{C}$, degrees Celsius; $\mathrm{g} / \mathrm{L}$, micrograms per liter; $\mathrm{S} / \mathrm{cm}$, microsiemens per centimeter at $25^{\circ} \mathrm{C} ;$ su, standard units. Symbol: --, no data].

\begin{tabular}{|c|c|c|c|c|c|c|c|c|}
\hline Date and time & $\begin{array}{c}\text { Streamflow, } \\
\text { instan- } \\
\text { taneous } \\
\left(\mathbf{f t}^{3} / \mathbf{s}\right)\end{array}$ & $\begin{array}{c}\text { Specific } \\
\text { conduc- } \\
\text { tance, } \\
\text { field } \\
(\mu S / \mathrm{cm})\end{array}$ & $\begin{array}{c}\text { pH, } \\
\text { field } \\
\text { (su) }\end{array}$ & $\begin{array}{c}\text { Temp- } \\
\text { erature, } \\
\text { water } \\
\left({ }^{\circ} \mathrm{C}\right)\end{array}$ & $\begin{array}{c}\text { Arsenic, } \\
\text { dissolved } \\
(\mu \mathrm{g} / \mathrm{L})\end{array}$ & $\begin{array}{c}\text { Cadmium, } \\
\text { dissolved } \\
(\mu \mathrm{g} / \mathrm{L})\end{array}$ & $\begin{array}{c}\text { Nickel, } \\
\text { dissolved } \\
(\mu \mathrm{g} / \mathrm{L})\end{array}$ & $\begin{array}{c}\text { Zinc, } \\
\text { dissolved } \\
(\mu \mathrm{g} / \mathrm{L})\end{array}$ \\
\hline $6 / 26 / 2001 \quad 4: 00$ & -- & 177 & 7.79 & 10.66 & 26.0 & 0.681 & 0.165 & 221 \\
\hline $6 / 26 / 2001 \quad 5: 00$ & 1.20 & 177 & 7.80 & 10.38 & 26.0 & 0.675 & 0.165 & 227 \\
\hline $6 / 26 / 2001 \quad 6: 00$ & 1.17 & 177 & 7.81 & 9.95 & 26.0 & 0.644 & 0.131 & 226 \\
\hline $6 / 26 / 2001 \quad 7: 00$ & 1.17 & 177 & 7.82 & 9.65 & 25.8 & 0.637 & 0.141 & 228 \\
\hline $6 / 26 / 2001 \quad 8: 00$ & 1.11 & 176 & 7.84 & 9.53 & 25.5 & 0.649 & 0.166 & 218 \\
\hline $6 / 26 / 2001 \quad 9: 00$ & 1.13 & 176 & 7.88 & 9.74 & 25.1 & 0.664 & 0.121 & 210 \\
\hline $6 / 26 / 2001$ 10:00 & 1.12 & 176 & 7.90 & 10.34 & 25.4 & 0.623 & 0.163 & 201 \\
\hline $6 / 26 / 2001$ 11:00 & 1.11 & 176 & 7.91 & 11.27 & 25.8 & 0.628 & 0.229 & 194 \\
\hline $6 / 26 / 2001$ 12:00 & 1.12 & 176 & 7.95 & 11.63 & 26.1 & 0.606 & 0.156 & 190 \\
\hline $6 / 26 / 200113: 00$ & 1.10 & 176 & 7.90 & 11.51 & 26.0 & 0.591 & 0.153 & 190 \\
\hline $6 / 26 / 2001$ 14:00 & 1.10 & 176 & 7.90 & 11.63 & 26.5 & 0.615 & 0.201 & 194 \\
\hline $6 / 26 / 2001$ 15:00 & 1.12 & 176 & 7.93 & 12.04 & 26.6 & 0.544 & 0.177 & 186 \\
\hline $6 / 26 / 2001$ 16:00 & 1.11 & 176 & 7.96 & 12.67 & 27.0 & 0.556 & 0.183 & 175 \\
\hline $6 / 26 / 2001$ 17:00 & 1.18 & 177 & 7.96 & 12.94 & 26.7 & 0.564 & 0.176 & 175 \\
\hline $6 / 26 / 2001$ 18:00 & 1.21 & 177 & 7.92 & 12.88 & 27.0 & 0.582 & 0.258 & 172 \\
\hline $6 / 26 / 2001$ 19:00 & 1.15 & 178 & 7.89 & 12.77 & 27.3 & 0.562 & 0.165 & 184 \\
\hline $6 / 26 / 200120: 00$ & 1.10 & 179 & 7.86 & 12.56 & 27.4 & 0.656 & 0.176 & 220 \\
\hline 6/26/2001 21:00 & -- & 179 & 7.84 & 12.42 & 26.3 & 0.654 & 0.245 & 217 \\
\hline $6 / 26 / 200122: 00$ & 1.16 & 179 & 7.80 & 12.23 & 26.2 & 0.648 & 0.141 & 224 \\
\hline $6 / 26 / 200123: 00$ & 1.12 & 180 & 7.80 & 12.00 & 26.0 & 0.637 & 0.171 & 207 \\
\hline $6 / 27 / 2001 \quad 0: 00$ & 1.13 & 180 & 7.79 & 11.80 & 25.6 & 0.666 & 0.142 & 224 \\
\hline $6 / 27 / 2001 \quad 1: 00$ & 1.13 & 181 & 7.80 & 11.74 & 26.1 & 0.664 & 0.149 & 223 \\
\hline $6 / 27 / 2001 \quad 2: 00$ & 1.13 & 181 & 7.78 & 11.60 & 25.9 & 0.681 & 0.183 & 228 \\
\hline $6 / 27 / 2001 \quad 3: 00$ & 1.13 & 181 & 7.78 & 11.48 & 25.7 & 0.654 & 0.097 & 231 \\
\hline $6 / 27 / 2001 \quad 4: 00$ & 1.12 & 181 & 7.76 & 11.33 & 25.6 & 0.654 & 0.184 & 225 \\
\hline $6 / 27 / 2001 \quad 5: 00$ & 1.11 & 181 & 7.77 & 11.13 & 25.5 & 0.749 & 0.147 & 220 \\
\hline $6 / 27 / 2001 \quad 6: 00$ & 1.14 & 181 & 7.79 & 10.87 & 26.0 & 0.676 & 0.308 & 229 \\
\hline $6 / 27 / 2001 \quad 7: 00$ & 1.14 & 181 & 7.80 & 10.65 & 26.3 & 0.664 & -- & 211 \\
\hline $6 / 27 / 2001 \quad 8: 00$ & 1.17 & 180 & 7.80 & 10.55 & 26.0 & 0.649 & 0.158 & 216 \\
\hline $6 / 27 / 2001 \quad 9: 00$ & 1.18 & 180 & 7.84 & 10.76 & 26.0 & 0.616 & 0.137 & 200 \\
\hline $6 / 27 / 2001$ 10:00 & 1.15 & 180 & 7.87 & 11.41 & 25.8 & 0.619 & 0.193 & 193 \\
\hline $6 / 27 / 200111: 00$ & 1.16 & 181 & 7.91 & 12.37 & 26.3 & 0.606 & 0.141 & 180 \\
\hline $6 / 27 / 2001$ 12:00 & 1.16 & 181 & 7.93 & 13.49 & 26.8 & 0.580 & 0.080 & 170 \\
\hline $6 / 27 / 2001$ 13:00 & 1.16 & 181 & 7.95 & 15.13 & 27.3 & 0.561 & 0.248 & 146 \\
\hline $6 / 27 / 200114: 00$ & 1.19 & 182 & 7.97 & 16.21 & 28.1 & 0.569 & 0.130 & 156 \\
\hline $6 / 27 / 2001$ 15:00 & 1.18 & 182 & 7.96 & 16.70 & 28.8 & 0.570 & 0.096 & 155 \\
\hline $6 / 27 / 2001$ 16:00 & 1.12 & 182 & 7.94 & 16.51 & 27.8 & 0.549 & 0.189 & 145 \\
\hline $6 / 27 / 2001$ 17:00 & 1.13 & 182 & 7.92 & 15.54 & 29.0 & 0.552 & 0.087 & 162 \\
\hline 6/27/2001 18:00 & 1.13 & 183 & 7.93 & 15.30 & 28.8 & 0.573 & 0.161 & 164 \\
\hline $6 / 27 / 2001$ 19:00 & 1.14 & 183 & 7.91 & 15.16 & 29.0 & 0.572 & 0.146 & 173 \\
\hline $6 / 27 / 200120: 00$ & 1.14 & 183 & 7.88 & 14.97 & 29.3 & 0.616 & 0.140 & 188 \\
\hline
\end{tabular}




\section{BED SEDIMENT DATA FOR STREAMS IN THE UPPER PRICKLY PEAR CREEK WATERSHED, MONTANA, 2001}

By Terry L. Klein and David L. Fey

\section{INTRODUCTION}

This section of the report presents the descriptions of sample sites and geochemical data for streambed and lakebed sediment samples collected by the USGS during July 2001. The locations of 35 streambed sediment sites are shown in figure 1. Samples were collected at these 35 sites to supplement data collected in October 2000 (Klein and others, 2001) and to characterize temporal geochemical variation by re-sampling at select October 2000 sites. Field duplicate samples were collected during October 2000 and July 2001 at fourteen sites and are indicated with the suffix " $r$ " in tables 8, 9, and 11 .

Chemical concentrations in streambed and lakebed sediments represent: 1) the composition of the underlying bedrock and glacial debris at and above the sample site 2) input from natural or cultural sources, for example naturally outcropping mineral deposits, or abandoned or active mines and mills. These sediments present a time-integrated view of the chemical input from these sources. Stream water chemistry is related to the composition of the underlying sediment through a variety of chemical solubility relationships, most of them governed by the $\mathrm{pH}$ (acidity) and Eh (oxidation potential) of the water. One of the most important sources of metal in water is metal loosely held in colloidal sediment material and grain coatings such as manganese and iron oxy-hydroxides. Streambed and lakebed sediment composition is also important because it may directly affect the health and metal content of benthic fauna, which are a primary food source for fish.

The locations of 35 streambed sediment sites from the July 2001 sample-collection program are shown in figure 1. Samples were collected at these 35 sites to supplement data from the October 2000 sample-collection program (Klein and others, 2001) and to determine temporal geochemical variation by re-sampling at select October 2000 sites. Samples were collected during October 2000 and July 2001 at fourteen sites and are indicated with the suffix " $r$ " in tables 8, 9, and 11 .

Each streambed sediment sample collected during July 2001 was analyzed using two different digestion methods (see below). These same methods were also used for streambed sediment samples collected in the watershed during 2000 (Klein and others, 2001). A four acid total-digestion method was used to determine the amount of metal within mineral grains that comprise the sediment and metal present in grain coatings. A dilute acid-peroxide partialdigestion method was used to determine the amount of loosely held metals (leachable) that are present mainly in grain coatings and colloidal material.

Lakebed sediment cores were collected from a small reservoir in the upper part of Lump Gulch, about 0.5 miles below the outlet of Frohner Meadows (fig. 1). A total of 6 sites were selected to determine the extent of metal accumulation in the reservoir. The locations of the 6 sample sites are shown in figure 2.

\section{SAMPLE AND ANALYTICAL PROTOCOL}

\section{Sample Locations}

A summary of field observations and locations of streambed sediment samples is given in table 8. Site locations from the July 2001 sampling program were determined with a Global Positioning System (GPS) onsite using a NAD 27 datum and subsequently transferred into a Geographic Information Systems (GIS) layer. Digital raster graphics (DRG) topographic maps were registered to the GIS layer and the GPS locations were checked against the topography for 
accuracy. Several GPS site locations were revised upon comparison with the DRG topographic maps.

All samples were collected within the upper Prickly Pear Creek watershed, except for two sites downstream from the USGS streamflow gaging station 06061500 (fig. 1). Sample sites are located on the Chessman Reservoir, Clancy, Jefferson City, Wickes, East Helena, Elkhorn, and Mount Thompson, Montana USGS 1:24,000 topographic maps.

\section{Streambed Sediments}

A composited streambed sediment sample was collected from the active alluvial channel at each site by combining 10 to 20 individual sub-sites within $15 \mathrm{~m}(50 \mathrm{ft}$.) of the plotted site. Each composite sample was sieved onsite through a $2 \mathrm{~mm}(10$-mesh) stainless steel screen, and the less than $2 \mathrm{~mm}$ fraction was retained. These site locations and field measurements are shown in table 8 .

Streambed sediment samples were air-dried at ambient room temperature $\left(25^{\circ} \mathrm{C}\right)$ and sieved to less than 80 -mesh $(<0.18 \mathrm{~mm})$ prior to laboratory analyses. Streambed sediment data are in tables 9 and 11.

\section{Lakebed Sediments}

A total of 6 lakebed sediment cores were collected from an unnamed reservoir in upper Lump Gulch (fig. 2). The cores were collected with a free-falling coring apparatus operated from a floating platform. Sample locations were determined with a GPS onsite using a NAD27 datum. Cores were described in the laboratory and subintervals were sampled. The material was air-dried at ambient room temperature $\left(25^{\circ} \mathrm{C}\right)$ and sieved to less than 80 -mesh $(<0.18 \mathrm{~mm})$. The sieved material was then pulverized to less than 100 -mesh $(<0.23 \mathrm{~mm})$ before laboratory analysis. Cores from sites 1-4 and site 6 were chemically analyzed. Lakebed site locations and sediment data are in table 10 .

\section{SAMPLE ANALYSIS}

\section{Total digestion}

Streambed and lakebed sediment samples were digested with a mixed-acid procedure consisting of $\mathrm{HCl}, \mathrm{HNO}_{3}, \mathrm{HClO}_{4}$, and $\mathrm{HF}$. Results are reported for 34 elements analyzed by inductively coupled plasma-atomic emission spectroscopy (ICP-AES) (Crock and others, 1983; Briggs, 1996). This procedure is effective in completely dissolving most minerals, including silicates, oxides and sulfides; and partially dissolving resistant or refractory minerals such as zircon, chromite, and some tin oxides. Previous investigations using a variety of materials support the completeness of the total digestion (Church and others, 1987; Wilson and others, 1994). Limits of determination for the total digestion method are given in table 5 of Fey and others (1999a). A statistical summary of mean values, standard deviations, and median values for four National Institute of Standards and Technology (NIST) standard reference materials (SRM-2704, SRM2709, SRM-2710, and SRM-2711) analyzed with samples from the Boulder River watershed Abandoned Mine Lands Initiative study is contained in table 6 of Fey and others, (1999a). Comparisons with certified values for these standards (NIST, 1993a, 1993b, 1993c and 1993d) are also in Fey and others, (1999a). Streambed and lakebed sediment data for the upper Prickly Pear Creek watershed using the total digestion method are shown in tables 9 and 10, respectively. 
Figure 2. Sketch map showing approximate locations of lakebed sediment sample sites in an unnamed reservoir in upper Lump Gulch. Location of reservoir is shown in fig. 1 as site $L G P$.

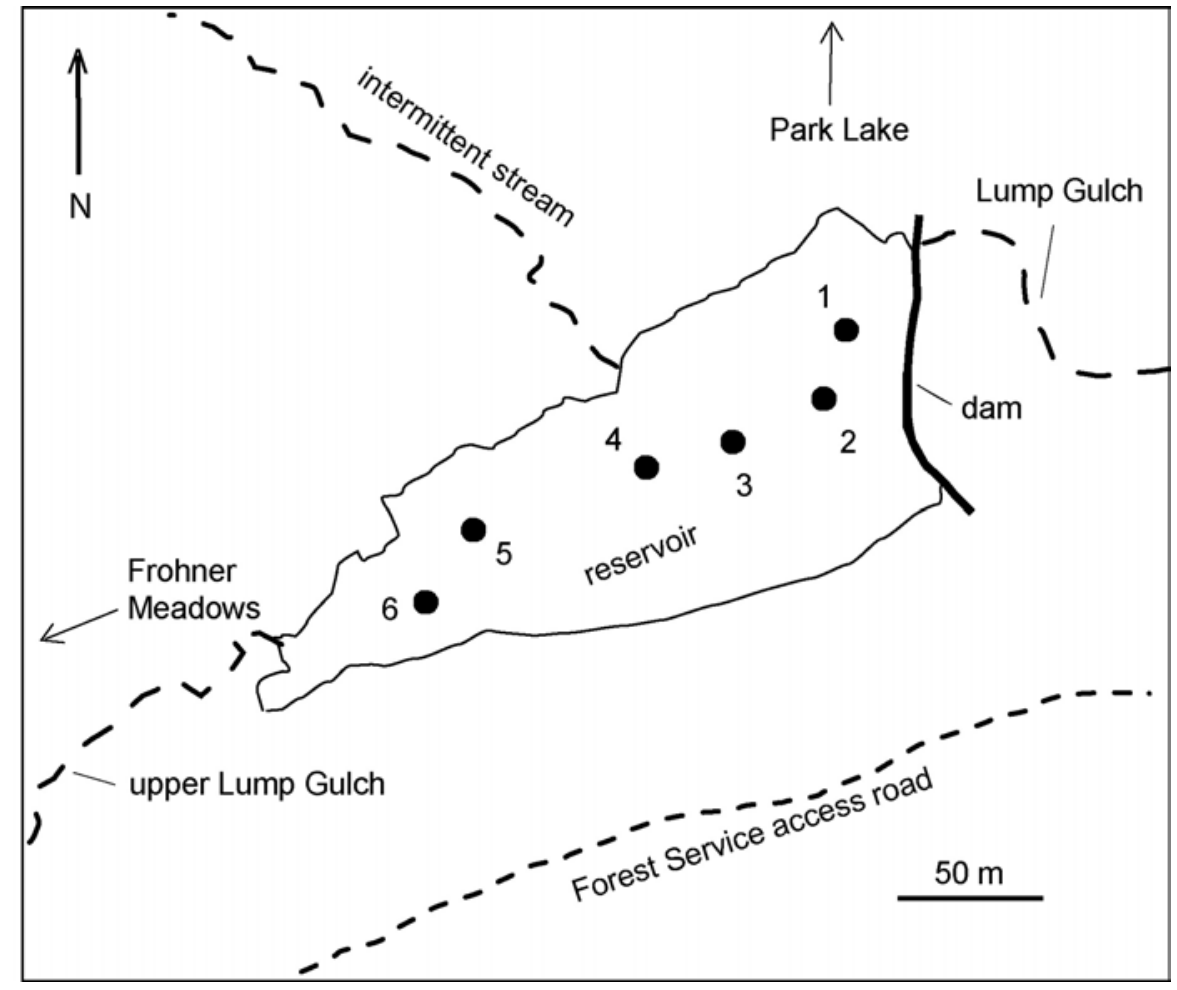

\section{Partial digestion}

Partial-digestion extraction method was used to determine concentrations of trace elements bound within different mineral phases (Chao, 1984). The streambed sediments were subjected to a partial-digestion extraction consisting of warm $\left(50^{\circ} \mathrm{C}\right) 2 \mathrm{M} \mathrm{HCl}-1$ percent $\mathrm{H}_{2} \mathrm{O}_{2}$ for three hours with continuous agitation; the leachates were subsequently analyzed by ICP-AES for 32 elements. This partial digestion extraction releases trace elements associated with hydrous amorphous ironand manganese-oxide mineral coatings and colloidal particles (Appendix III of Church and others, 1993; Church and others, 1997). Mineral coatings, such as those observed in the study area, can contain a significant percentage of the trace elements in a sample (Church and others, 1997; Fey and Church, 1998; Fey and others, 1999b; 2000). Analytical limits of determination for the partial-digestion extraction method are also in table 5 of Fey and others (1999a). A statistical summary of mean values, standard deviations, and median values obtained using the partial digestion method for the same four National Institute of Standards and Technology standard reference materials (SRM-2704, SRM-2709, SRM-2710, and SRM-2711) is in table 8 of Fey and others (1999a). Streambed sediment data for the upper Prickly Pear Creek watershed using the partial digestion method are shown in table 11.

\section{Field measurements of water samples}

Conductivity and $\mathrm{pH}$ of stream water samples were measured onsite using temperaturecompensated instruments calibrated daily with solutions of the appropriate range. The results, expressed as micro-siemens/centimeter $(\mu \mathrm{S} / \mathrm{cm})$ and standard $\mathrm{pH}$ units are in table 8 . Sediment sample sites located on streams that were not flowing are noted in table 8 . 
Table 8. Streambed sediment site locations and field measurements of water samples in the upper Prickly Pear Creek watershed, Montana, July 2001.

[Samples collected during July 2001 by David Fey and Paul Wigton, USGS. Latitude and longitude were determined with a Global Positioning System using a horizontal NAD27 datum. Replicate samples collected at a site during October, 2000 and July, 2001 are marked with ' $r$ ' in the site number.]

\begin{tabular}{|c|c|c|c|c|c|c|}
\hline Site No & sample description & $\begin{array}{l}\text { latitude } \\
(\mathbf{N})\end{array}$ & $\begin{array}{l}\text { longitude } \\
\text { (W) }\end{array}$ & pH & $\begin{array}{c}\text { conductivity } \\
\mu \mathrm{S} / \mathrm{cm}\end{array}$ & date \\
\hline C-3r & sand and gravel & 46.38556 & -112.14139 & 6.92 & 121 & $7 / 22 / 2001$ \\
\hline C-4r & cobbles, gravel & 46.40222 & -112.11944 & 7.21 & 210 & $7 / 22 / 2001$ \\
\hline C-100r & sand & 46.38889 & -112.11861 & 3.10 & 1140 & $7 / 22 / 2001$ \\
\hline C-102r & sand and gravel & 46.41028 & -112.1475 & 7.36 & 100 & $7 / 20 / 2001$ \\
\hline C-103r & sand and gravel & 46.40833 & -112.14917 & 7.44 & 140 & $7 / 20 / 2001$ \\
\hline $\mathrm{C}-200$ & cobbles, gravel, sand & 46.40517 & -112.18416 & 6.98 & 113 & $7 / 20 / 2001$ \\
\hline C-201 & sand & 46.39225 & -112.12103 & 7.1 & 202 & $7 / 22 / 2001$ \\
\hline C-202 & sand and gravel & 46.39779 & -112.12126 & 7.44 & 420 & $7 / 22 / 2001$ \\
\hline C-203 & cobbles, gravel & 46.38768 & -112.15697 & 7.15 & 123 & $7 / 22 / 2001$ \\
\hline C-204 & cobbles, gravel & 46.38824 & -112.15729 & no & flow & 7/22/2001 \\
\hline C-205 & sand and gravel & 46.44012 & -112.06093 & 7.48 & 238 & 7/23/2001 \\
\hline D-1r & sand and gravel & 46.41669 & -111.99973 & 7.17 & 84 & $7 / 23 / 2001$ \\
\hline L-3br & sand & 46.43667 & -112.18790 & 6.15 & 97 & 7/13/2001 \\
\hline L-4r & sand & 46.43684 & -112.17223 & 6.97 & 75 & 7/13/2001 \\
\hline L-4ar & sand & 46.43541 & -112.17673 & 6.96 & 69 & 7/13/2001 \\
\hline L-100r & sand & 46.44302 & -112.19855 & 6.26 & 66 & $7 / 15 / 2001$ \\
\hline L-150 (L-3a) $)^{1}$ & sand & 46.43411 & -112.48955 & 6.00 & 81 & $7 / 13 / 2001$ \\
\hline L-151 & sand & 46.43395 & -112.18934 & 6.25 & 53 & 7/13/2001 \\
\hline L-152 & sand and silt & 46.43847 & -112.17259 & 6.58 & 78 & 7/13/2001 \\
\hline L-153 & sand and gravel & 46.43676 & -112.17419 & no & flow & $7 / 13 / 2001$ \\
\hline L-154 & sand and gravel & 46.43531 & -112.17749 & 7.17 & 67 & 7/13/2001 \\
\hline L-155 & sand and gravel & 46.43524 & -112.18047 & 7.17 & 67 & 7/13/2001 \\
\hline L-156 & sand and gravel & 46.43628 & -112.18438 & 6.90 & 67 & $7 / 13 / 2001$ \\
\hline L-157 & sand and gravel & 46.43504 & -112.18664 & 7.07 & 56 & $7 / 13 / 2001$ \\
\hline L-158 & sand and gravel & 46.43665 & -112.18594 & 6.84 & 96 & $7 / 13 / 2001$ \\
\hline L-159 & sand & 46.43664 & -112.19405 & 6.56 & 121 & $7 / 14 / 2001$ \\
\hline L-160 & fine organic material and peat & 46.43623 & -112.19144 & 6.48 & 123 & 7/14/2001 \\
\hline L-161 & sand and gravel & 46.43842 & -112.19408 & 6.60 & 206 & 7/14/2001 \\
\hline L-162 & sand and gravel & 46.44163 & -112.20333 & 6.04 & 70 & $7 / 15 / 2001$ \\
\hline L-163 & sand & 46.44323 & -112.19746 & 6.20 & 69 & $7 / 15 / 2001$ \\
\hline L-164 & sand & 46.44292 & -112.19551 & 6.45 & 69 & $7 / 15 / 2001$ \\
\hline L-165 & sand & 46.44229 & -112.19487 & 6.51 & 73 & $7 / 15 / 2001$ \\
\hline L-166 & sand & 46.44073 & -112.19516 & 6.39 & 99 & $7 / 15 / 2001$ \\
\hline L-167 & sand & 46.44072 & -112.19659 & 6.41 & 520 & $7 / 15 / 2001$ \\
\hline L-168 & sand & 46.43932 & -112.19451 & 5.30 & 111 & $7 / 15 / 2001$ \\
\hline L-180 & sand & 46.47858 & -111.98283 & 7.59 & 167 & $7 / 20 / 2001$ \\
\hline PP-2a & sand & 46.37832 & -112.02994 & 7.26 & 101 & $7 / 22 / 2001$ \\
\hline PP-2b & sand and silt & 46.38956 & -112.02402 & 7.27 & 105 & $7 / 22 / 2001$ \\
\hline PP-3r & sand and gravel & 46.40194 & -112.01444 & 6.85 & 150 & $7 / 22 / 2001$ \\
\hline PP-3a & sand and gravel & 46.42062 & -111.99789 & 7.05 & 186 & $7 / 23 / 2001$ \\
\hline PP-3b & sand and gravel & 46.43714 & -111.99146 & 7.88 & 180 & $7 / 20 / 2001$ \\
\hline PP-4r & sand and gravel & 46.45500 & -111.98413 & 7.42 & 192 & 7/20/2001 \\
\hline PP-4a & sand and gravel & 46.4687 & -111.98222 & 7.67 & 198 & $7 / 20 / 2001$ \\
\hline PP-4b & sand and gravel & 46.48451 & -111.97166 & 7.68 & 200 & $7 / 20 / 2001$ \\
\hline PP-5r & sand and gravel & 46.51917 & -111.94611 & 7.88 & 202 & $7 / 20 / 2001$ \\
\hline PP-100 & sand & 46.40194 & -112.01977 & no & flow & $7 / 22 / 2001$ \\
\hline PP-200 & sand & 46.55427 & -111.90940 & 7.79 & 223 & $7 / 20 / 2001$ \\
\hline
\end{tabular}


Table 8. Field numbers, site descriptions, sample descriptions, latitude and longitude, and field measurements of streambed sediment localities. (Continued)

\begin{tabular}{|c|l|c|c|c|c|c|}
\hline Field No & \multicolumn{1}{|c|}{ sample description } & $\begin{array}{c}\text { latitude } \\
(\mathbf{N})\end{array}$ & \multicolumn{1}{c|}{$\begin{array}{c}\text { longitude } \\
\text { (W) }\end{array}$} & $\mathbf{p H}$ & conductivity & \multicolumn{1}{c|}{ date } \\
\hline PP-201 & sand & 46.53427 & -111.93513 & 7.69 & 214 & $7 / 20 / 2001$ \\
\hline S-3r & sand and gravel & 46.37632 & -112.06323 & 7.26 & 665 & $7 / 22 / 2001$ \\
\hline S-4r & sand and gravel & 46.38887 & -112.02716 & no & flow & $7 / 22 / 2001$ \\
\hline S-200 & sand & 46.38414 & -112.05098 & no & flow & $7 / 22 / 2001$ \\
\hline S-201 & sand & 46.38083 & -112.06126 & no & flow & $7 / 22 / 2001$ \\
\hline S-202 & sand & 46.34276 & -112.10922 & no & flow & $7 / 22 / 2001$ \\
\hline S-203 & sand & 46.36168 & -112.07643 & no & flow & $7 / 22 / 2001$ \\
\hline S-204 & sandy fluvial tailings & 46.35799 & -112.12746 & 6.97 & 556 & $7 / 23 / 2001$ \\
\hline S-205 & sand & 46.38300 & -112.05305 & 7.22 & 475 & $7 / 23 / 2001$ \\
\hline S-206 & sand & 46.38560 & -112.04090 & no & flow & $7 / 23 / 2001$ \\
\hline WS-8 & sand and gravel & 46.45002 & -111.98438 & 7.67 & 237 & $7 / 20 / 2001$ \\
\hline
\end{tabular}

${ }^{1}$ same location as site L-3a in table 1. 
Table 9. Streambed sediment data using the mixed-acid total digestion method for the upper Prickly Pear Creek watershed, Montana, July 2001.

[Analyzed by David Fey, USGS. The following elements with their detection limits (ppm) in parenthesis were analyzed but not detected: gold (8), bismuth (10), europium (2), holmium (4), tantalum (40), uranium (100). < is less than the value indicated, ' $r$ ' in the site number indicates that samples were collected at this site during October 2000 and July 2001 . Major element data expressed in

weight percent; trace element data expressed in ppm (parts per million), dry weight basis.]

\begin{tabular}{|c|c|c|c|c|c|c|c|c|c|c|c|c|c|c|c|c|c|c|}
\hline Site No. & Al \% & $\mathrm{Ca} \%$ & $\mathrm{Fe} \%$ & K \% & Mg \% & $\mathrm{Na} \%$ & P \% & $\mathrm{Ti} \%$ & Ag ppm & As ppm & Ba ppm & Be ppm & Cd ppm & Ce ppm & Co ppm & Cr ppm & Cu ppm & Ga ppm \\
\hline$C-3 r$ & 7.6 & 1.4 & 5.8 & 3.0 & 0.70 & 1.0 & 0.12 & 0.42 & $<2$ & 44 & 770 & 2 & 9 & 88 & 13 & 49 & 32 & 20 \\
\hline C-4r & 7.4 & 1.4 & 7.4 & 2.6 & 0.70 & 1.2 & 0.15 & 0.29 & 11 & 1,400 & 790 & 3 & 36 & 91 & 24 & 77 & 590 & 22 \\
\hline C-100r & 6.6 & 0.64 & 3.1 & 2.6 & 0.42 & 1.5 & 0.09 & 0.21 & 4 & 210 & 850 & 2 & 3 & 43 & 3 & 37 & 87 & 15 \\
\hline C-102r & 7.7 & 1.8 & 11 & 2.0 & 0.55 & 2.4 & 0.14 & 0.29 & $<2$ & 16 & 700 & 2 & 6 & 110 & 30 & 83 & 15 & 21 \\
\hline C-103r & 7.7 & 2.1 & 5.6 & 2.4 & 0.72 & 1.7 & 0.14 & 0.36 & $<2$ & 42 & 860 & 2 & 13 & 97 & 12 & 28 & 51 & 21 \\
\hline C-200 & 8.5 & 2.0 & 5.1 & 2.2 & 0.56 & 2.7 & 0.12 & 0.34 & $<2$ & 23 & 710 & 2 & 6 & 120 & 9 & 47 & 26 & 21 \\
\hline C-201 & 7.1 & 0.92 & 4.6 & 2.4 & 0.55 & 0.92 & 0.15 & 0.14 & 14 & 1,900 & 740 & 5 & 77 & 110 & 19 & 35 & 1900 & 24 \\
\hline C-202 & 7.1 & 2.0 & 14 & 2.0 & 0.54 & 2.1 & 0.22 & 0.38 & 9 & 160 & 750 & 2 & 10 & 150 & 35 & 110 & 74 & 27 \\
\hline C-203 & 7.8 & 1.2 & 5.0 & 3.2 & 0.68 & 0.90 & 0.12 & 0.36 & $<2$ & 51 & 770 & 2 & 7 & 80 & 13 & 39 & 34 & 20 \\
\hline C-204 & 7.3 & 1.9 & 8.9 & 2.4 & 1.0 & 1.2 & 0.13 & 0.65 & $<2$ & 31 & 710 & 2 & 39 & 110 & 21 & 86 & 220 & 24 \\
\hline C-205 & 7.6 & 2.7 & 5.6 & 2.6 & 1.1 & 1.6 & 0.15 & 0.50 & $<2$ & 15 & 610 & 2 & 4 & 88 & 22 & 57 & 38 & 18 \\
\hline D-1r & 7.3 & 2.6 & 11 & 1.9 & 0.71 & 2.4 & 0.16 & 0.52 & $<2$ & $<10$ & 610 & 2 & 6 & 200 & 26 & 74 & 29 & 20 \\
\hline L-3br & 8.6 & 1.4 & 4.9 & 1.9 & 0.25 & 2.8 & 0.09 & 0.11 & $<2$ & 640 & 1,000 & 3 & 90 & 55 & 77 & 4 & 140 & 24 \\
\hline L-4ar & 8.8 & 1.7 & 2.3 & 2.2 & 0.29 & 2.9 & 0.06 & 0.16 & $<2$ & 37 & 880 & 2 & 14 & 64 & $<1$ & 10 & 22 & 22 \\
\hline L-4r & 7.4 & 1.7 & 3.4 & 1.9 & 0.45 & 2.2 & 0.12 & 0.18 & $<2$ & 72 & 870 & 2 & 37 & 71 & 4 & 14 & 52 & 18 \\
\hline L-100r & 7.6 & 0.98 & 4.8 & 1.9 & 0.25 & 1.8 & 0.05 & 0.09 & 25 & 3,200 & 400 & 2 & 57 & 42 & 84 & 6 & 300 & 27 \\
\hline L-150 & 8.9 & 1.7 & 3.5 & 2.3 & 0.23 & 3.0 & 0.07 & 0.11 & $<2$ & 260 & 1,300 & 2 & 42 & 72 & 14 & 7 & 52 & 24 \\
\hline L-151 & 9.2 & 1.7 & 3.6 & 2.4 & 0.25 & 2.9 & 0.08 & 0.16 & $<2$ & 20 & 860 & 2 & 3 & 78 & $<1$ & 21 & 9 & 22 \\
\hline L-152 & 8.1 & 1.7 & 0.99 & 2.4 & 0.27 & 2.6 & 0.07 & 0.15 & $<2$ & 15 & 870 & 2 & 2 & 65 & $<1$ & 6 & 16 & 19 \\
\hline L-153 & 8.3 & 1.5 & 4.1 & 1.9 & 0.82 & 1.6 & 0.11 & 0.31 & $<2$ & 47 & 700 & 2 & 12 & 78 & 8 & 39 & 74 & 21 \\
\hline L-154 & 8.6 & 1.6 & 3.9 & 2.1 & 0.35 & 2.9 & 0.08 & 0.19 & $<2$ & 58 & 900 & 2 & 32 & 95 & 6 & 19 & 37 & 23 \\
\hline L-155 & 8.6 & 1.6 & 4.6 & 2.1 & 0.43 & 2.5 & 0.10 & 0.16 & $<2$ & 130 & 1,000 & 2 & 52 & 90 & 35 & 18 & 52 & 24 \\
\hline L-156 & 9.0 & 1.7 & 4.7 & 2.0 & 0.49 & 2.8 & 0.09 & 0.23 & $<2$ & 86 & 800 & 2 & 20 & 73 & 8 & 36 & 65 & 22 \\
\hline L-157 & 9.1 & 1.7 & 5.0 & 2.3 & 0.29 & 3.1 & 0.10 & 0.18 & $<2$ & 26 & 840 & 2 & 6 & 98 & 3 & 31 & 15 & 23 \\
\hline L-158 & 9.5 & 1.7 & 5.1 & 2.0 & 0.43 & 2.8 & 0.11 & 0.17 & $<2$ & 340 & 990 & 3 & 58 & 86 & 64 & 14 & 98 & 27 \\
\hline L-159 & 8.6 & 1.3 & 2.9 & 1.9 & 0.24 & 2.6 & 0.05 & 0.11 & 2 & 900 & 860 & 2 & 44 & 45 & 25 & 6 & 160 & 22 \\
\hline L-160 & 7.6 & 1.4 & 5.7 & 1.7 & 0.20 & 2.4 & 0.09 & 0.08 & 3 & 2,500 & 940 & 3 & 80 & 50 & 240 & 2 & 280 & 21 \\
\hline L-161 & 8.9 & 1.2 & 4.2 & 2.4 & 0.44 & 2.6 & 0.07 & 0.17 & 2 & 790 & 830 & 2 & 58 & 48 & 44 & 16 & 130 & 22 \\
\hline L-162 & 7.5 & 0.82 & 3.3 & 2.2 & 0.25 & 1.6 & 0.05 & 0.10 & 34 & 3,300 & 720 & 2 & 22 & 36 & 44 & 5 & 190 & 23 \\
\hline L-163 & 8.2 & 1.1 & 5.1 & 2.0 & 0.27 & 2.0 & 0.06 & 0.10 & 24 & 3,300 & 820 & 3 & 53 & 46 & 99 & 4 & 280 & 26 \\
\hline L-164 & 9.2 & 1.4 & 2.9 & 2.2 & 0.28 & 2.7 & 0.06 & 0.15 & 6 & 1,000 & 900 & 2 & 10 & 64 & 4 & 12 & 130 & 25 \\
\hline L-165 & 9.8 & 1.6 & 3.0 & 2.2 & 0.33 & 3.0 & 0.06 & 0.17 & 12 & 2,100 & 900 & 2 & 15 & 54 & 12 & 8 & 220 & 26 \\
\hline L-166 & 8.0 & 1.1 & 2.7 & 2.0 & 0.27 & 2.2 & 0.05 & 0.12 & 20 & 2,000 & 710 & 2 & 19 & 34 & $<1$ & 6 & 300 & 21 \\
\hline
\end{tabular}


Table 9. Streambed sediment data using the total digestion method for the upper Prickly Pear Creek watershed, Montana, July 2001. (Continued)

\begin{tabular}{|c|c|c|c|c|c|c|c|c|c|c|c|c|c|c|c|c|}
\hline Field No. & La ppm & Li ppm & Mn ppm & Mo ppm & Nb ppm & Nd ppm & \begin{tabular}{|l|} 
Ni ppm \\
\end{tabular} & Pb ppm & Sc ppm & Sn ppm & Sr ppm & Th ppm & \begin{tabular}{|l|l|} 
V ppm \\
\end{tabular} & Y ppm & Yb ppm & Zn ppm \\
\hline C-3r & 52 & 17 & 4,600 & 4 & 24 & 32 & 19 & 200 & 11 & $<5$ & 290 & 26 & 170 & 21 & 2 & 1,100 \\
\hline C-4r & 54 & 21 & 7,900 & 4 & $<4$ & 37 & 30 & 1,300 & 12 & 6 & 290 & 17 & 220 & 30 & 3 & 5,800 \\
\hline C-100r & 25 & 18 & 440 & 2 & 38 & 20 & 10 & 330 & 6 & 15 & 320 & 10 & 70 & 12 & 1 & 310 \\
\hline C-102r & 63 & 18 & 620 & $<2$ & 44 & 47 & 15 & 29 & 9 & $<5$ & 460 & 32 & 340 & 25 & 3 & 89 \\
\hline C-103r & 59 & 20 & 8,200 & 3 & $<4$ & 36 & 19 & 160 & 12 & $<5$ & 390 & 22 & 120 & 27 & 3 & 2,200 \\
\hline C-200 & 76 & 20 & 900 & 2 & 53 & 49 & 12 & 64 & 9 & $<5$ & 550 & 23 & 160 & 26 & 3 & 390 \\
\hline C-201 & 81 & 21 & 19,000 & 4 & $<4$ & 44 & 42 & 2,200 & 10 & 7 & 250 & 9 & 88 & 65 & 5 & 9,800 \\
\hline C-202 & 86 & 15 & 920 & 3 & 37 & 59 & 18 & 130 & 11 & 6 & 430 & 44 & 450 & 29 & 4 & 600 \\
\hline C-203 & 47 & 18 & 6,100 & 5 & 13 & 28 & 19 & 270 & 11 & $<5$ & 280 & 21 & 130 & 19 & 2 & 1,000 \\
\hline C-204 & 61 & 21 & 8,000 & 4 & 8 & 43 & 34 & 230 & 16 & $<5$ & 270 & 37 & 320 & 41 & 4 & 4,000 \\
\hline C-205 & 50 & 24 & 1,100 & 2 & 50 & 37 & 19 & 34 & 15 & $<5$ & 340 & 24 & 200 & 28 & 3 & 130 \\
\hline D-1r & 120 & 13 & 730 & 5 & 82 & 80 & 14 & 19 & 14 & 7 & 460 & 170 & 340 & 49 & 6 & 54 \\
\hline L-3br & 37 & 25 & 10,000 & 5 & $<4$ & 20 & 17 & 320 & 5 & $<5$ & 620 & 10 & 42 & 20 & 2 & 5,600 \\
\hline L-4ar & 40 & 18 & 890 & 2 & 44 & 25 & 7 & 56 & 4 & $<5$ & 650 & 17 & 50 & 12 & 2 & 1,100 \\
\hline L-4r & 44 & 28 & 4,300 & 5 & 15 & 31 & 14 & 58 & 8 & $<5$ & 470 & 15 & 58 & 27 & 3 & 2,800 \\
\hline L-100r & 23 & 49 & 18,000 & 7 & $<4$ & 6 & 19 & 3,800 & 4 & 5 & 420 & 5 & 40 & 17 & 2 & 4,400 \\
\hline L-150r & 45 & 15 & 9,000 & 11 & $<4$ & 23 & 13 & 130 & 4 & $<5$ & 680 & 11 & 49 & 15 & 2 & 3,200 \\
\hline L-151 & 49 & 20 & 570 & 3 & 48 & 32 & 7 & 37 & 4 & $<5$ & 640 & 24 & 100 & 14 & 2 & 120 \\
\hline L-152 & 41 & 18 & 680 & 2 & 46 & 25 & 5 & 31 & 4 & $<5$ & 560 & 15 & 23 & 18 & 2 & 180 \\
\hline L-153 & 43 & 46 & 1,600 & 3 & 43 & 34 & 20 & 110 & 13 & $<5$ & 350 & 29 & 110 & 30 & 3 & 1,500 \\
\hline L-154 & 60 & 21 & 2,700 & 3 & 34 & 36 & 11 & 70 & 6 & $<5$ & 600 & 20 & 87 & 19 & 2 & 2,500 \\
\hline L-155 & 58 & 26 & 8,300 & 5 & $<4$ & 33 & 18 & 110 & 7 & $<5$ & 540 & 18 & 86 & 25 & 3 & 5,200 \\
\hline L-156 & 47 & 27 & 1,400 & 3 & 44 & 31 & 12 & 110 & 7 & $<5$ & 600 & 26 & 120 & 19 & 2 & 1,800 \\
\hline L-157 & 63 & 20 & 530 & 3 & 45 & 37 & 8 & 57 & 5 & $<5$ & 630 & 26 & 140 & 16 & 2 & 470 \\
\hline L-158 & 54 & 30 & 8,900 & 6 & $<4$ & 30 & 16 & 220 & 7 & $<5$ & 670 & 16 & 90 & 20 & 2 & 4,700 \\
\hline L-159 & 32 & 27 & 3,000 & 4 & 22 & 21 & 10 & 1,200 & 4 & $<5$ & 560 & 12 & 27 & 20 & 2 & 2,400 \\
\hline L-160 & 36 & 23 & 12,000 & 9 & $<4$ & 22 & 20 & 1,300 & 5 & $<5$ & 520 & 7 & 29 & 31 & 3 & 6,600 \\
\hline L-161 & 31 & 30 & 4,000 & 4 & 19 & 18 & 12 & 720 & 6 & $<5$ & 520 & 29 & 79 & 16 & 2 & 2,600 \\
\hline L-162 & 21 & 58 & 10,000 & 6 & $<4$ & 9 & 11 & 5,400 & 4 & $<5$ & 370 & 11 & 39 & 12 & 1 & 2,000 \\
\hline L-163 & 27 & 55 & 17,000 & 6 & $<4$ & 10 & 19 & 3,700 & 5 & 6 & 470 & 8 & 46 & 18 & 2 & 4,300 \\
\hline L-164 & 40 & 32 & 2,000 & 8 & 36 & 25 & 8 & 1,500 & 4 & $<5$ & 610 & 13 & 62 & 12 & 1 & 830 \\
\hline L-165 & 36 & 40 & 2,400 & 4 & 38 & 23 & 9 & 2,000 & 5 & $<5$ & 650 & 14 & 47 & 15 & 2 & 1,400 \\
\hline L-166 & 22 & 36 & 750 & 3 & 36 & 16 & 7 & 2,400 & 4 & 7 & 470 & 9 & 34 & 11 & 1 & 1,800 \\
\hline
\end{tabular}


Table 9. Streambed sediment data using the total digestion method for the upper Prickly Pear Creek watershed, Montana, July 2001. (Continued)

\begin{tabular}{|c|c|c|c|c|c|c|c|c|c|c|c|c|c|c|c|c|c|c|}
\hline Field No. & Al \% & $\mathrm{Ca} \%$ & $\mathrm{Fe} \%$ & K \% & Mg \% & $\mathrm{Na} \%$ & P \% & $\mathrm{Ti} \%$ & Ag ppm & As ppm & Ba ppm & Be ppm & Cd ppm & Ce ppm & Co ppm & Cr ppm & Cu ppm & Ga ppm \\
\hline L-167 & 9.0 & 1.9 & 4.2 & 2.3 & 0.66 & 2.2 & 0.11 & \begin{tabular}{|l|}
0.26 \\
\end{tabular} & $<2$ & 410 & 690 & 3 & 24 & 82 & 34 & 14 & 180 & 22 \\
\hline L-168 & 7.4 & 1.1 & 1.8 & 1.7 & 0.23 & 2.2 & 0.04 & 0.11 & $<2$ & 580 & 710 & 2 & 19 & 39 & 11 & 4 & 100 & 19 \\
\hline L-180 & 6.9 & 2.8 & 17 & 1.8 & 0.83 & 2.2 & \begin{tabular}{|l|}
0.19 \\
\end{tabular} & \begin{tabular}{|l|}
0.40 \\
\end{tabular} & $<2$ & 14 & 590 & 2 & 10 & 140 & 61 & 120 & 13 & 24 \\
\hline PP-2a & 7.7 & 3.1 & 5.8 & 2.6 & 1.3 & 2.2 & 0.16 & \begin{tabular}{|l|}
0.48 \\
\end{tabular} & $<2$ & 15 & 580 & 2 & 5 & 150 & 12 & 84 & 39 & 18 \\
\hline PP-2b & 6.1 & 1.4 & 4.0 & 2.0 & 0.72 & 1.0 & 0.11 & \begin{tabular}{|l|}
0.22 \\
\end{tabular} & 19 & 340 & 580 & 2 & 25 & 62 & 6 & 31 & 500 & 19 \\
\hline PP-3a & 7.1 & 2.4 & 9.8 & 2.1 & 0.77 & 2.2 & 0.16 & \begin{tabular}{|l|}
0.50 \\
\end{tabular} & 2 & 59 & 630 & 2 & 8 & 200 & 23 & 75 & 89 & 20 \\
\hline PP-3b & 7.4 & 2.5 & 7.8 & 2.2 & 0.89 & 2.1 & \begin{tabular}{|l|}
0.17 \\
\end{tabular} & 0.45 & 8 & 170 & 620 & 2 & 22 & 170 & 19 & 56 & 230 & 20 \\
\hline PP-3r & 6.5 & 1.7 & 5.6 & 2.2 & 0.77 & 1.4 & 0.14 & \begin{tabular}{|l|}
0.32 \\
\end{tabular} & 13 & 360 & 570 & 2 & 15 & 83 & 12 & 46 & 440 & 19 \\
\hline PP-4a & 6.6 & 2.4 & 14 & 2.0 & 0.70 & 2.0 & 0.17 & \begin{tabular}{|l|}
0.58 \\
\end{tabular} & 3 & 120 & 640 & 2 & 13 & 220 & 32 & 100 & 120 & 22 \\
\hline PP-4b & 7.2 & 2.6 & 9.5 & 2.1 & 0.69 & 2.3 & 0.15 & 0.41 & $<2$ & 43 & 610 & 2 & 8 & 180 & 20 & 68 & 63 & 22 \\
\hline PP-4r & 8.0 & 2.5 & 5.2 & 2.4 & 0.94 & 2.2 & 0.16 & \begin{tabular}{|l|}
0.38 \\
\end{tabular} & 6 & 120 & 620 & 2 & 12 & 110 & 14 & 38 & 170 & 21 \\
\hline PP-5r & 7.2 & 2.7 & 12 & 2.0 & 0.86 & 2.2 & \begin{tabular}{|l|}
0.21 \\
\end{tabular} & \begin{tabular}{|l|}
0.48 \\
\end{tabular} & 2 & 45 & 600 & 2 & 9 & 160 & 41 & 120 & 54 & 21 \\
\hline PP-100 & 8.1 & 2.1 & 6.6 & 2.1 & 0.63 & 2.0 & \begin{tabular}{|l|}
0.18 \\
\end{tabular} & \begin{tabular}{|l|}
0.28 \\
\end{tabular} & $<2$ & 14 & 730 & 2 & 5 & 110 & 19 & 54 & 92 & 21 \\
\hline PP-200 & 7.6 & 3.9 & 7.5 & 2.2 & 1.3 & 2.2 & 0.20 & 0.48 & $<2$ & 49 & 580 & 2 & 6 & 170 & 16 & 80 & 54 & 16 \\
\hline PP-201 & 7.5 & 2.9 & 5.9 & 2.2 & 0.94 & 2.2 & 0.15 & 0.38 & $<2$ & 31 & 580 & 2 & 5 & 110 & 12 & 61 & 45 & 18 \\
\hline S-3r & 7.6 & 1.6 & 4.6 & 2.7 & 0.46 & 2.0 & \begin{tabular}{|l|}
0.10 \\
\end{tabular} & \begin{tabular}{|l|}
0.27 \\
\end{tabular} & 6 & 300 & 870 & 2 & 11 & 69 & 6 & 35 & 260 & 20 \\
\hline S-4r & 5.6 & 1.3 & 6.2 & 2.0 & 0.51 & 0.95 & \begin{tabular}{|l|}
0.12 \\
\end{tabular} & \begin{tabular}{|l|}
0.22 \\
\end{tabular} & 38 & 720 & 620 & 2 & 41 & 82 & 8 & 43 & 940 & 24 \\
\hline S-200 & 7.9 & 2.1 & 6.3 & 2.3 & 1.4 & 1.3 & 0.21 & 0.49 & $<2$ & 52 & 600 & 2 & 8 & 98 & 33 & 47 & 180 & 21 \\
\hline S-201 & 8.9 & 2.6 & 3.5 & 3.0 & 1.1 & 2.3 & 0.16 & \begin{tabular}{|l|}
0.38 \\
\end{tabular} & $<2$ & 27 & 790 & 2 & 4 & 79 & 12 & 22 & 180 & 20 \\
\hline S-202 & 8.2 & 1.6 & 1.6 & 2.6 & 0.46 & 2.3 & 0.08 & 0.17 & $<2$ & 42 & 960 & 2 & 4 & 33 & $<1$ & 21 & 66 & 21 \\
\hline S-203 & 7.5 & \begin{tabular}{|l|}
0.85 \\
\end{tabular} & 5.2 & 3.2 & 0.42 & 1.4 & \begin{tabular}{|l|}
0.12 \\
\end{tabular} & \begin{tabular}{|l|}
0.17 \\
\end{tabular} & $<2$ & 38 & 790 & 2 & 4 & 46 & 5 & 20 & 280 & 17 \\
\hline S-204 & 5.0 & 0.60 & 13 & 2.0 & 0.42 & 0.51 & 0.07 & 0.16 & 19 & 12,000 & 170 & $<1$ & 30 & 26 & 60 & 25 & 220 & 16 \\
\hline S-205 & 8.1 & 1.6 & 5.4 & 2.8 & 0.92 & 1.6 & 0.11 & 0.31 & 3 & 89 & 920 & 2 & 12 & 72 & 15 & 52 & 190 & 21 \\
\hline S-206 & 7.8 & 1.7 & 4.7 & 2.4 & 0.94 & 1.5 & 0.13 & 0.33 & 4 & 120 & 740 & 2 & 13 & 74 & 19 & 55 & 240 & 19 \\
\hline WS-8 & 7.7 & 2.8 & 4.4 & 2.2 & 0.89 & 2.4 & 0.17 & \begin{tabular}{|l|}
0.37 \\
\end{tabular} & $<2$ & 40 & 460 & 3 & 4 & 120 & 8 & 29 & 25 & 18 \\
\hline
\end{tabular}


Table 9. Streambed sediment data using the total digestion method for the upper Prickly Pear Creek watershed, Montana, July 2001. (Continued)

\begin{tabular}{|c|c|c|c|c|c|c|c|c|c|c|c|c|c|c|c|c|}
\hline Field No. & La ppm & Li ppm & Mn ppm & Mo ppm & Nb ppm & Nd ppm & Ni ppm & Pb ppm & Sc ppm & Sn ppm & Sr ppm & Th ppm & V ppm & $\mathbf{Y}$ ppm & Yb ppm & Zn ppm \\
\hline L-167 & 50 & 28 & 2,800 & 22 & 47 & 32 & 12 & 490 & 10 & $<5$ & 440 & 29 & 95 & 26 & 3 & 3,500 \\
\hline L-168 & 26 & 22 & 1,200 & 3 & 32 & 17 & 6 & 500 & 4 & $<5$ & 500 & 8 & 24 & 12 & 1 & 1,600 \\
\hline L-180 & 80 & 14 & 790 & 3 & 46 & 68 & 19 & 9 & 16 & 5 & 410 & 110 & 550 & 44 & 6 & 130 \\
\hline PP-2a & 86 & 15 & 1,200 & 3 & 75 & 60 & 21 & 98 & 17 & $<5$ & 420 & 54 & 170 & 43 & 5 & 280 \\
\hline PP-2b & 35 & 27 & 10,000 & 6 & $<4$ & 21 & 19 & 2,800 & 9 & 8 & 250 & 17 & 82 & 22 & 2 & 3,800 \\
\hline PP-3a & 120 & 16 & 1,800 & 4 & 64 & 74 & 15 & 390 & 14 & 5 & 430 & 180 & 310 & 44 & 5 & 570 \\
\hline PP-3b & 98 & 20 & 2,600 & 4 & 53 & 66 & 15 & 610 & 15 & 8 & 430 & 120 & 230 & 43 & 5 & 3,000 \\
\hline PP-3r & 48 & 25 & 6,900 & 7 & 4 & 31 & 17 & 1,800 & 11 & 9 & 310 & 34 & 150 & 26 & 3 & 2,600 \\
\hline PP-4a & 130 & 15 & 2,200 & 4 & 63 & 79 & 17 & 360 & 14 & 8 & 360 & 140 & 440 & 48 & 6 & 1,200 \\
\hline PP-4b & 110 & 13 & 1,600 & 3 & 63 & 69 & 14 & 150 & 13 & 5 & 430 & 95 & 300 & 43 & 5 & 640 \\
\hline PP-4r & 68 & 25 & 3,000 & 4 & 46 & 44 & 15 & 560 & 14 & $<5$ & 450 & 49 & 140 & 32 & 4 & 1,900 \\
\hline PP-5r & 92 & 18 & 1,700 & 4 & 64 & 65 & 19 & 220 & 15 & $<5$ & 420 & 82 & 380 & 44 & 5 & 680 \\
\hline PP-100 & 66 & 33 & 600 & 3 & 47 & 42 & 16 & 71 & 10 & 5 & 440 & 41 & 200 & 25 & 3 & 180 \\
\hline PP-200 & 100 & 18 & 1,700 & 3 & 76 & 70 & 17 & 170 & 16 & $<5$ & 450 & 72 & 240 & 49 & 6 & 600 \\
\hline PP-201 & 60 & 18 & 1,700 & 2 & 50 & 45 & 14 & 180 & 13 & 10 & 420 & 46 & 180 & 34 & 4 & 630 \\
\hline S-3r & 40 & 18 & 1,300 & 4 & 42 & 28 & 13 & 890 & 8 & $<5$ & 480 & 13 & 110 & 14 & 2 & 1,700 \\
\hline S-4r & 47 & 26 & 19,000 & 5 & $<4$ & 21 & 17 & 4,900 & 8 & 7 & 250 & 16 & 140 & 24 & 3 & 6,900 \\
\hline S-200 & 52 & 40 & 1,900 & 4 & 42 & 41 & 25 & 370 & 20 & 5 & 280 & 30 & 180 & 32 & 4 & 930 \\
\hline S-201 & 44 & 26 & 930 & 4 & 61 & 36 & 16 & 95 & 15 & $<5$ & 520 & 18 & 90 & 27 & 3 & 240 \\
\hline S-202 & 20 & 30 & 570 & $<2$ & 43 & 15 & 9 & 260 & 5 & $<5$ & 620 & 6 & 31 & 10 & 1 & 240 \\
\hline S-203 & 29 & 12 & 240 & 78 & 36 & 21 & 9 & 110 & 8 & 6 & 350 & 16 & 81 & 10 & 1 & 130 \\
\hline S-204 & 14 & 19 & 2,400 & 2 & $<4$ & 16 & 22 & 2,900 & 10 & 12 & 160 & 7 & 82 & 7 & $<1$ & 4,400 \\
\hline S-205 & 38 & 24 & 3,000 & 5 & 22 & 29 & 19 & 520 & 12 & $<5$ & 430 & 14 & 130 & 19 & 2 & 1,300 \\
\hline S-206 & 42 & 26 & 3,200 & 5 & 24 & 30 & 24 & 590 & 12 & 5 & 380 & 15 & 120 & 22 & 2 & 1,600 \\
\hline WS-8 & 70 & 20 & 660 & 3 & 72 & 51 & 10 & 44 & 14 & 6 & 410 & 78 & 130 & 40 & 5 & 260 \\
\hline
\end{tabular}


Table 10. Lakebed sediment site locations and data using the mixed-acid total digestion method for upper Prickly Pear Creek watershed, Montana, July 2001.

[Samples were collected by David Fey, Terry Klein and Paul Wigton, USGS and analyzed by David Fey. Depths are measured from top of recovered core and are not corrected for compaction. The following

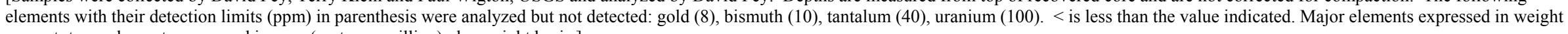
percent; trace elements expressed in ppm (parts per million), dry weight basis.]

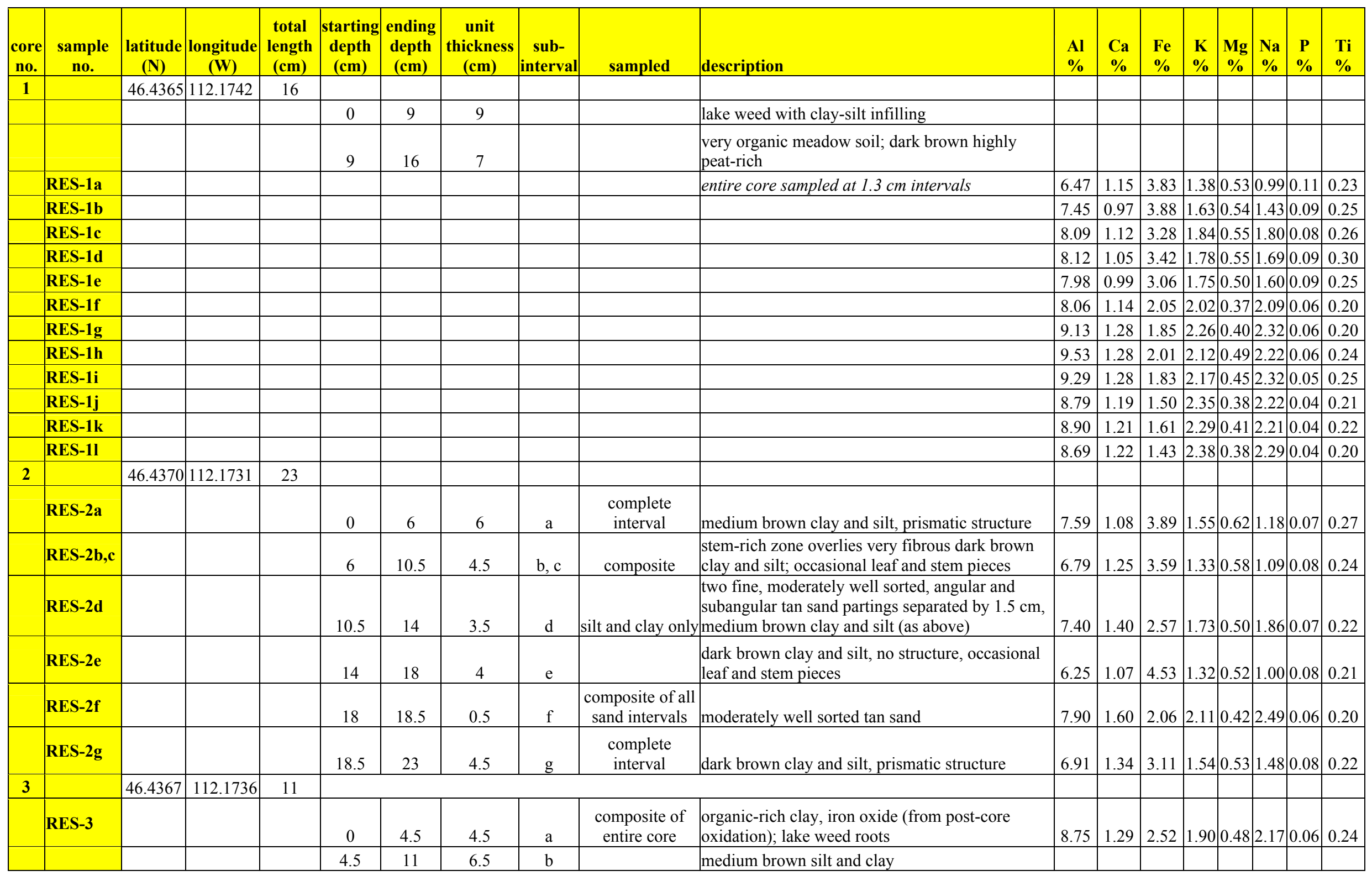


Table 10. Core descriptions and locations, and the total digestion ICP-AES results from lakebed sediments collected July 2001 from the upper Lump Gulch (Continued).

\begin{tabular}{|c|c|c|c|c|c|c|c|c|c|c|c|c|c|c|c|c|c|c|c|c|c|c|c|c|c|c|c|c|c|}
\hline $\begin{array}{c}\text { core } \\
\text { no. }\end{array}$ & sample no. & $\begin{array}{c}\text { Ag } \\
\text { ppm }\end{array}$ & $\begin{array}{c}\text { As } \\
\text { ppm }\end{array}$ & $\begin{array}{c}\text { Ba } \\
\text { ppm }\end{array}$ & $\begin{array}{c}\mathrm{Be} \\
\mathbf{p p m}\end{array}$ & $\begin{array}{c}\text { Cd } \\
\text { ppm }\end{array}$ & $\begin{array}{c}\mathrm{Ce} \\
\mathrm{ppm}\end{array}$ & $\begin{array}{c}\text { Co } \\
\text { ppm }\end{array}$ & $\begin{array}{c}\mathrm{Cr} \\
\mathbf{p p m}\end{array}$ & $\begin{array}{c}\mathrm{Cu} \\
\mathbf{p p m}\end{array}$ & $\begin{array}{c}\mathbf{E u} \\
\mathbf{p p m}\end{array}$ & $\begin{array}{c}\text { Ga } \\
\text { ppm }\end{array}$ & \begin{tabular}{|c|} 
Ho \\
ppm
\end{tabular} & $\begin{array}{c}\mathbf{L a} \\
\mathbf{p p m}\end{array}$ & $\begin{array}{c}\mathbf{L i} \\
\mathbf{p p m}\end{array}$ & $\begin{array}{c}\text { Mn } \\
\text { ppm }\end{array}$ & $\begin{array}{c}\text { Mo } \\
\text { ppm }\end{array}$ & $\begin{array}{c}\mathrm{Nb} \\
\mathrm{ppm}\end{array}$ & \begin{tabular}{c|} 
Nd \\
ppm
\end{tabular} & $\begin{array}{c}\mathrm{Ni} \\
\text { ppm }\end{array}$ & \begin{tabular}{c|}
$\mathbf{P b}$ \\
$\mathbf{p p m}$
\end{tabular} & $\begin{array}{c}\text { Sc } \\
\text { ppm }\end{array}$ & $\begin{array}{c}\text { Sn } \\
\text { ppm }\end{array}$ & $\begin{array}{c}\mathrm{Sr} \\
\mathbf{p p m}\end{array}$ & \begin{tabular}{|c|} 
Th \\
ppm
\end{tabular} & $\begin{array}{c}\mathrm{V} \\
\mathbf{p p m}\end{array}$ & $\begin{array}{c}\mathbf{Y} \\
\mathbf{p p m}\end{array}$ & $\begin{array}{c}\text { Yb } \\
\text { ppm }\end{array}$ & $\begin{array}{c}\mathbf{Z n} \\
\mathbf{p p m}\end{array}$ \\
\hline \multicolumn{30}{|l|}{1} \\
\hline & & & & & & & & & & & & & & & & & & & & & & & & & & & & & \\
\hline & & & & & & & & & & & & & & & & & & & & & & & & & & & & & \\
\hline & RES-1a & 2 & 202 & 478 & 1.8 & 54 & 66 & 21 & 17 & 102 & 1.1 & 16 & 1.1 & 41 & 40 & 1,083 & 5 & 18 & 34 & 17 & 165 & 9 & 5 & 239 & 18 & 62 & 31 & 3 & 7,537 \\
\hline & RES-1b & 2 & 181 & 690 & 2.0 & 38 & 72 & 18 & 17 & 101 & 1.1 & 19 & 1.2 & 45 & 41 & 893 & 3 & 20 & 37 & 15 & 189 & 9 & 2 & 299 & 19 & 66 & 31 & 3 & 4,412 \\
\hline & RES-1c & 1 & 144 & 797 & 2.0 & 25 & 73 & 15 & 15 & 89 & 1.2 & 21 & 1.2 & 45 & 39 & 748 & 2 & 23 & 35 & 12 & 166 & 9 & 4 & 373 & 18 & 64 & 30 & 3 & 2,419 \\
\hline & RES-1d & 1 & 158 & 813 & 2.1 & 28 & 80 & 17 & 17 & 106 & 1.3 & 21 & 1.2 & 50 & 41 & 773 & 3 & 28 & 40 & 13 & 157 & 10 & 4 & 351 & 18 & 67 & 34 & 3 & 2,472 \\
\hline & RES-1e & 1 & 137 & 785 & 2.1 & 28 & 77 & 12 & 16 & 103 & 1.3 & 21 & 1.3 & 48 & 41 & 730 & 2 & 25 & 38 & 12 & 145 & 9 & 4 & 334 & 18 & 63 & 33 & 3 & 2,219 \\
\hline & RES-1f & 1 & 62 & 887 & 2.0 & 14 & 68 & 3 & 12 & 55 & 1.2 & 20 & 1.0 & 42 & 32 & 446 & 2 & 27 & 32 & 9 & 85 & 6 & 1 & 442 & 15 & 47 & 25 & 2 & 1,364 \\
\hline & RES-1g & 1 & 14 & 954 & 2.2 & 7 & 85 & $<2$ & 13 & 44 & 1.5 & 23 & 1.2 & 51 & 37 & 473 & 1 & 28 & 41 & 8 & 67 & 7 & 3 & 489 & 16 & 49 & 32 & 3 & 720 \\
\hline & RES-1h & 1 & 9 & 901 & 2.2 & 4 & 76 & $<2$ & 16 & 36 & 1.3 & 24 & 1.1 & 47 & 44 & 487 & 1 & 29 & 36 & 9 & 70 & 9 & 3 & 475 & 17 & 56 & 28 & 3 & 401 \\
\hline & RES-1i & 1 & 11 & 920 & 2.0 & 4 & 69 & $<2$ & 14 & 34 & 1.2 & 23 & 1.2 & 42 & 40 & 426 & 0 & 30 & 31 & 8 & 60 & 8 & 2 & 480 & 18 & 51 & 24 & 3 & 398 \\
\hline & RES-1j & 1 & 16 & 964 & 2.0 & 3 & 59 & $<2$ & 13 & 31 & 1.1 & 22 & 0.8 & 36 & 34 & 344 & 1 & 28 & 27 & 8 & 59 & 6 & 3 & 475 & 13 & 43 & 20 & 2 & 258 \\
\hline & RES-1k & 1 & 15 & 935 & 2.1 & 4 & 70 & $<2$ & 16 & 42 & 1.2 & 23 & 1.3 & 44 & 35 & 380 & 1 & 30 & 33 & 9 & 63 & 7 & 2 & 480 & 14 & 49 & 24 & 2 & 258 \\
\hline & RES-11 & 1 & 13 & 950 & 2.1 & 3 & 65 & $<2$ & 13 & 45 & 1.2 & 22 & 0.9 & 40 & 32 & 367 & 2 & 28 & 29 & 9 & 60 & 6 & 4 & 484 & 12 & 44 & 22 & 2 & 246 \\
\hline \multicolumn{30}{|l|}{2} \\
\hline & RES-2a & 2 & 149 & 230 & 1.8 & 26 & 71 & 24 & 21 & 116 & 1.0 & 20 & 1.0 & 44 & 51 & 978 & 6 & 23 & 35 & 17 & 185 & 10 & 3 & 264 & 20 & 75 & 32 & 3 & 3,149 \\
\hline & RES-2b,c & 2 & 205 & 260 & 1.7 & 27 & 74 & 25 & 22 & 141 & 1.0 & 17 & 1.2 & 44 & 49 & 1,177 & 10 & 18 & 36 & 17 & 136 & 10 & 4 & 262 & 21 & 74 & 34 & 3 & 2,500 \\
\hline & RES-2d & 1 & 101 & 661 & 1.8 & 17 & 77 & 6 & 17 & 90 & 1.0 & 19 & 1.1 & 44 & 38 & 823 & 3 & 23 & 33 & 11 & 112 & 8 & 3 & 405 & 19 & 63 & 29 & 3 & 1,292 \\
\hline & RES-2e & 2 & 288 & 532 & 1.9 & 40 & 73 & 33 & 18 & 155 & 1.1 & 16 & 1.1 & 44 & 42 & 2,464 & 4 & 11 & 36 & 17 & 183 & 10 & 3 & 243 & 19 & 72 & 35 & 3 & 3,367 \\
\hline & RES-2f & 1 & 64 & 807 & 1.8 & 12 & 66 & 6 & 10 & 51 & 0.8 & 19 & 0.9 & 37 & 27 & 555 & 3 & 26 & 27 & 9 & 78 & 6 & 2 & 522 & 15 & 43 & 20 & 2 & 1,072 \\
\hline & RES-2g & 1 & 164 & 439 & 1.8 & 27 & 73 & 23 & 18 & 115 & 0.9 & 17 & 1.2 & 43 & 41 & 1,511 & 4 & 19 & 32 & 14 & 120 & 9 & 0 & 338 & 18 & 65 & 30 & 3 & 2,425 \\
\hline \multicolumn{30}{|l|}{3} \\
\hline & RES-3 & 1 & 78 & 818 & 2.0 & 14 & 75 & 5 & 16 & 72 & 1.1 & 22 & 1.1 & 47 & 39 & 504 & 3 & 25 & 35 & 11 & 115 & 8 & 1 & 458 & 17 & 60 & 27 & 3 & 1,339 \\
\hline & & & & & & & & & & & & & & & & & & & & & & & & & & & & & \\
\hline
\end{tabular}


Table 10. Core descriptions and locations, and the total digestion ICP-AES results from lakebed sediments collected July 2001 from the upper Lump Gulch. (Continued)

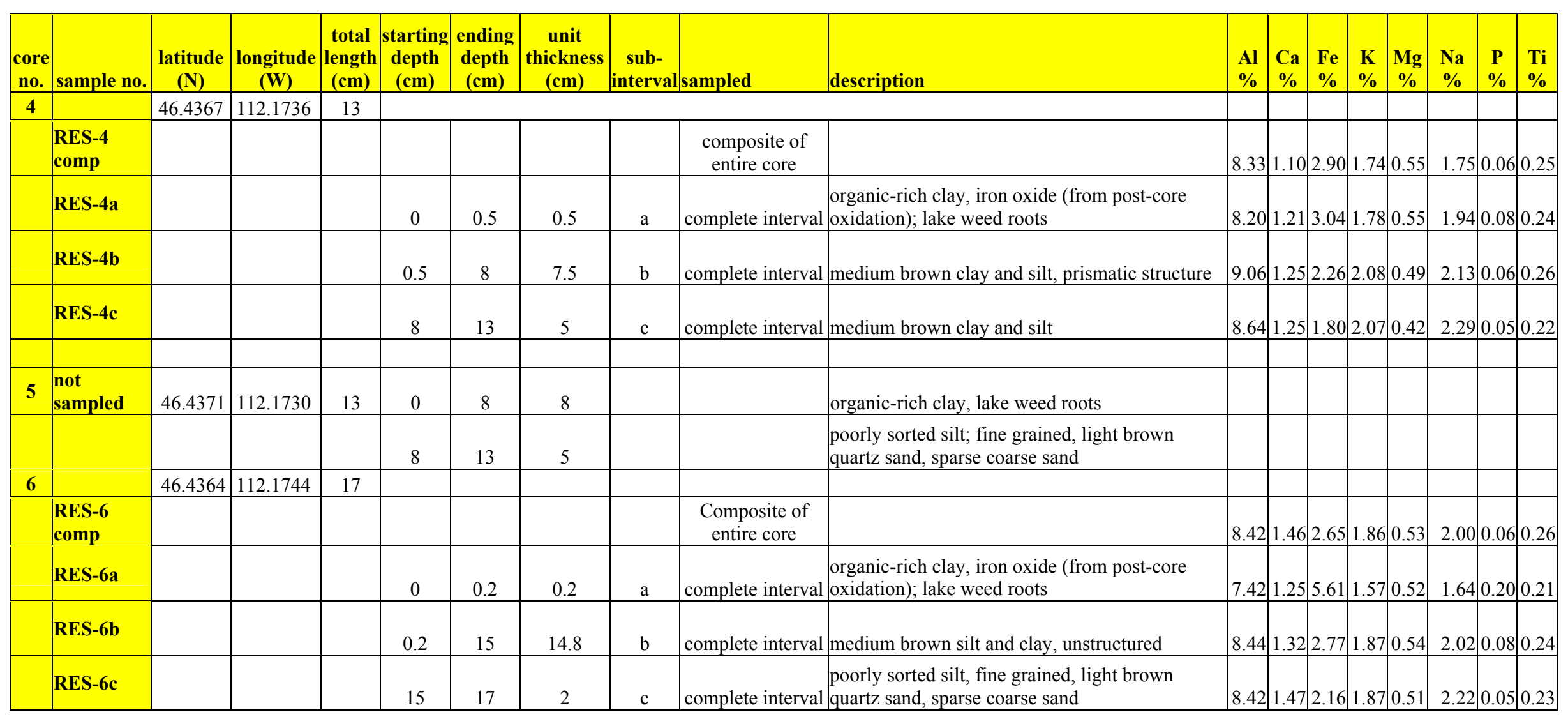


Table 10. Core descriptions and locations, and the total digestion ICP-AES results from lakebed sediments collected July 2001 from the upper Lump Gulch. (Continued)

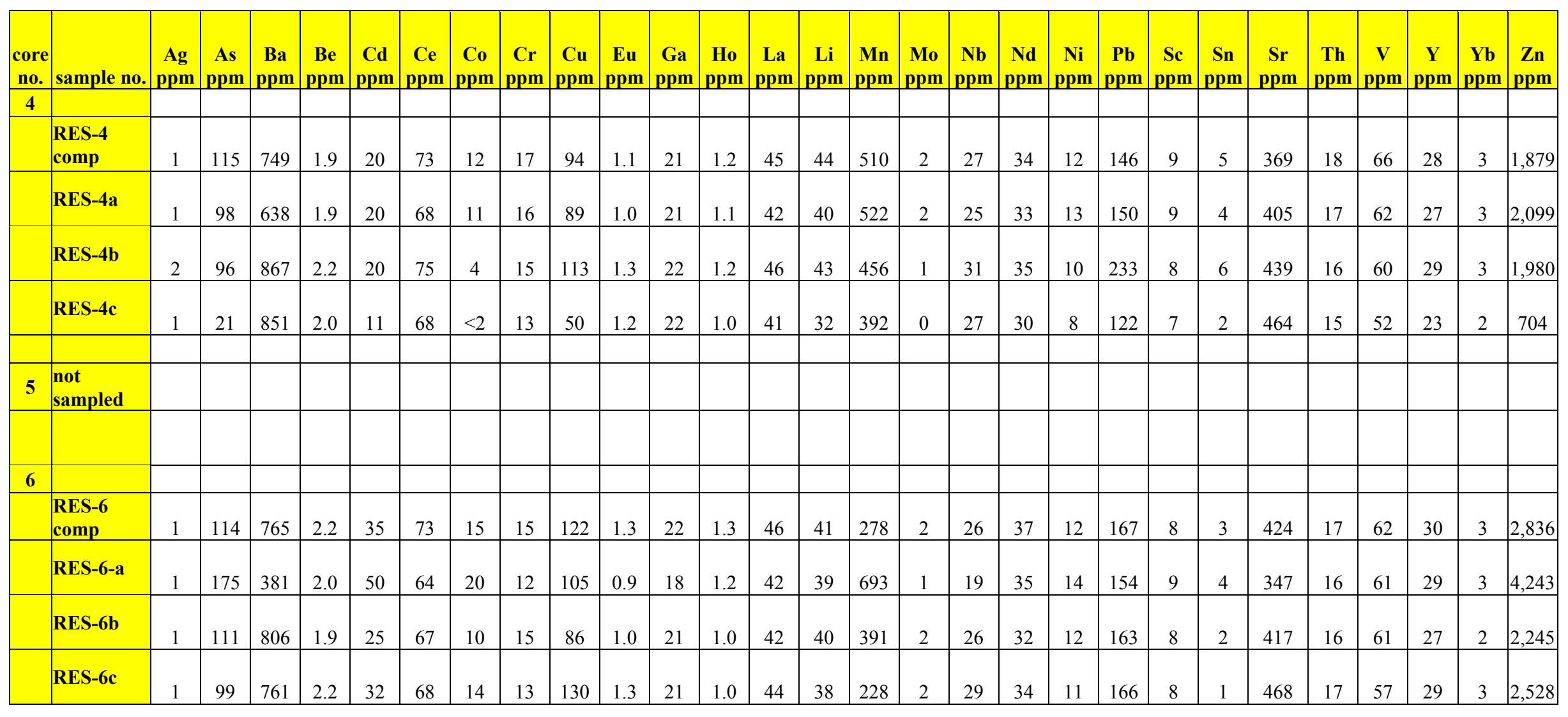


Table 11. Streambed sediment data using the partial digestion method for the upper Prickly Pear Creek watershed, Montana, July 2001.

Analyzed by David Fey, USGS. All values in ppm. < is less than the indicated value.

\begin{tabular}{|c|c|c|c|c|c|c|c|c|c|c|c|c|c|c|c|c|c|c|c|c|c|c|c|c|c|c|c|}
\hline & Al & a & 10 & K & $g$ & $\mathbf{N a}$ & $\mathbf{P}$ & SI & $\mathbf{T i}$ & Ag & As & & $\mathbf{B a}$ & $\mathrm{Be}$ & Cd & Co & $\mathrm{Cr}$ & $\mathbf{C u}$ & $\mathbf{L i}$ & Mn & Mo & $\mathrm{Ni}$ & $\mathbf{P b}$ & $\mathbf{S b}$ & $\mathbf{r}$ & V & n \\
\hline$C_{-} 3 r$ & 00 & 000 & 000 & 860 & 00 & 64 & 870 & 00 & 110 & & - & & 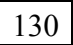 & 6 & 9 & 2 & & 2 & 33 & 000 & 2 & 3 & 130 & $<3$ & 30 & 14 & 80 \\
\hline & & & & 1,000 & & 72 & 100 & & $<30$ & 10 & & & & & 30 & 14 & 9 & 450 & 40 & 100 & $<1$ & 19 &, 000 & $<3$ & 8 & 15 & \\
\hline $0 \mathrm{ar}$ & & & & & & 63 & 600 & & 40 & & & & & & & 40 & $-\sigma_{1}$ & 35 & & & 1 & 3.0 & 200 & $<3$ & 4 & 86 & 60 \\
\hline & 00 & 00 & 00 & & 00 & 81 & 00 & 00 & 320 & & & & & 0.6 & $<1$ & 10 & 3.4 & 1 & & & 1 & 4.2 & 24 & 3 & -1 & 18 & \\
\hline & 400 & 000 & 000 & & 300 & 51 & 94 & 500 & 110 & 1 & & 3 & 0 & 0.6 & \begin{tabular}{|l|}
8.6 \\
\end{tabular} & 10 & & 8 & - & 000 & 1 & 10 & 00 & $<3$ & 2 & 6 & 500 \\
\hline & 900 & 00 & 00 & $3 x$ & 1,200 & OS & 850 & & 10 & $<1$ & & 3 & & 0.6 & 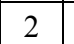 & \begin{tabular}{|l|}
5.3 \\
\end{tabular} & 3.0 & 3 & & 50 & $<1$ & .1 & 34 & $<3$ & 1 & 12 & 40 \\
\hline & 400 & 600 & 000 & 440 & 900 & 38 & 710 & 400 & $<30$ & 8.9 & 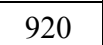 & 3 & & & \begin{tabular}{|l|}
46 \\
\end{tabular} & 11 & & 970 & & 2,000 & $<1$ & 22 & 100 & $<3$ & 29 & 8 & 300 \\
\hline & 800 & 700 & 00 & 1,100 & 100 & 63 & 000 & 1,200 & 270 & 2 & & $<3$ & & $=0$ & & \begin{tabular}{|l|}
5.9 \\
\end{tabular} & 3.4 & 37 & & \begin{tabular}{|l|}
600 \\
\end{tabular} & & 1.2 & 120 & $<3$ & 16 & 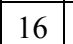 & 30 \\
\hline & 400 & 600 & 000 & 1,100 & 200 & 71 & \begin{tabular}{|l|}
870 \\
\end{tabular} & 300 & 100 & $<1$ & & $<3$ & & & \begin{tabular}{|l|}
4.9 \\
\end{tabular} & 8.8 & & 20 & & 0 & & 10 & 00 & $<3$ & 2 & 16 & 20 \\
\hline & 600 & 00 & & 70 & 300 & 40 & 820 & 0 & \begin{tabular}{|l|}
72 \\
\end{tabular} & 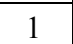 & & 3 & 0 & & 27 & 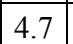 & - & 0 & & 0 & 1 & 17 & \begin{tabular}{|l|}
30 \\
\end{tabular} & $<3$ & 0 & \begin{tabular}{|l|}
22 \\
\end{tabular} &, 0 \\
\hline & 300 & & & 000 & 800 & 100 & 1,000 & 1,100 & 480 & $<1$ & & 6 & 10 & 0.6 & $<1$ & 9.5 & 4.9 & $2 J$ & & & $<1$ & .8 & -2 & $<3$ & 33 & 19 & $\pi$ \\
\hline & 000 & 00 & & 680 & 400 & 80 & 1,200 & 970 & 230 & $<1$ & & 3 & & $<0.6$ & \begin{tabular}{|l|}
$<1$ \\
\end{tabular} & 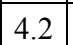 & & \begin{tabular}{|l|}
8.8 \\
\end{tabular} & & & & & & $<3$ & 25 & 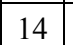 & 19 \\
\hline & 200 & & & & & 4 & 600 & 950 & $<30$ & - & & & & & 7 & 61 & 0.6 & & & & & 2 & 20 & 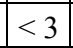 & 0 & & \\
\hline & $\mathrm{C}$ & & & & 100 & & $\overline{43}$ & & \begin{tabular}{|l|}
87 \\
\end{tabular} & 41 & & & & $<0$. & \begin{tabular}{|l|l}
11 \\
\end{tabular} & \begin{tabular}{|l|}
6.5 \\
\end{tabular} & 0.6 & & & & & & & 3 & 5 & 1.1 & \\
\hline & 3,500 & & 000 & 40 & 00 & 49 & 69 & 880 & 86 & $<1$ & & & 0 & 0.6 & \begin{tabular}{|l|}
28 \\
\end{tabular} & \begin{tabular}{|l|}
8.2 \\
\end{tabular} & 0.8 & & & $0_{1}$ & & .1 & $\delta$ & $<3$ & 41 & 12 & 00 \\
\hline & 00 & 00 & 0 & & 470 & 30 & 28 & 00 & $<30$ & 19 & 10 & $<$ & 210 & & 46 & 83 & $<0.6$ & 200 & 3.2 & 6,000 & 3.1 & 14 & 2,900 & $<<3$ & 37 & 5.7 & 3,500 \\
\hline & 200 & 0 & 0 & & 880 & 40 & 62 & 1,200 & $<30$ & $<1$ & & $<3$ & 90 & 0.6 & 40 & \begin{tabular}{|l|}
26 \\
\end{tabular} & $<0.6$ & 47 & & 00 & 8.1 & 8.5 & 120 & $<3$ & 29 & 9 & 3, \\
\hline & 300 & - & & 28 & 880 & 63 & 60 & & 50 & $<1$ & & $<3$ & & $<0.6$ & \begin{tabular}{|l|}
$<1$ \\
\end{tabular} & 4.8 & & \begin{tabular}{|l|}
8.0 \\
\end{tabular} & & & 1 & & 18 & $<3$ & 19 & 7.7 & - \\
\hline & 000 & 0 & & 18 & 710 & 47 & 46 & 30 & 120 & $<1$ & & $<3$ & & $<0.6$ & & & 0.9 & 10 & & & $<$ & & 2 & $<3$ & 17 & 4.3 & \\
\hline & 600 & 0 & 00 & 1,30 & 500 & 59 & 63 & 0 & 190 & 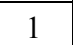 & & $<3$ & 60 & 0.6 & \begin{tabular}{|l|}
9.5 \\
\end{tabular} & 11 & 1 & 41 & 8.7 & 0 & 1 & 8.5 & 7 & $<3$ & 53 & \begin{tabular}{|l|}
34 \\
\end{tabular} & \\
\hline & 000 & 0 & & 490 & 80 & 44 & 63 & 0 & \begin{tabular}{|l|}
86 \\
\end{tabular} & $<1$ & & $<3$ & 0 & $<0.6$ & 27 & 13 & 0 & 29 & 3.9 & & $<1$ & .1 & 46 & $<3$ & 24 & \begin{tabular}{|l|}
12 \\
\end{tabular} & \\
\hline & 00 & & & & 1,400 & 45 & 56 & & 30 & $<1$ & & $<3$ & 20 & & 38 & 28 & 9 & 32 & & & & .7 & 1 & $<3$ & 4 & 15 & \\
\hline & 300 & & & 5 & 1,600 & 69 & 63 & 1,000 & \begin{tabular}{|l|}
98 \\
\end{tabular} & $<1$ & & $<3$ & 120 & $<0.6$ & 15 & 13 & & 31 & & 0 & $<$ & 4.2 & 3 & $<3$ & 6 & 12 & 1,400 \\
\hline & 000 & & & 2 & 870 & 55 & 61 & & 61 & $<1$ & & $<3$ & & $<0.6$ & 3 & 3.3 & 0.9 & \begin{tabular}{|l|}
8.7 \\
\end{tabular} & .0 & & & 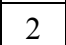 & 27 & $<3$ & 15 & 7.4 & 0 \\
\hline & 800 & 0 & & 18 & 49 & 63 & 56 & 1,200 & $<30$ & 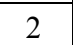 & 2,10 & 4.6 & & 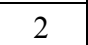 & \begin{tabular}{|l|}
76 \\
\end{tabular} & 180 & $<0.6$ & 220 & & & 3.6 & 16 & 1,000 & $<3$ & 36 & 0 & 5,900 \\
\hline & 200 & & & & 48 & 41 & 36 & & $<30$ & 2 & & - & & & 38 & 35 & $<0.6$ & & & & & 8 & $\begin{array}{l}960 \\
\end{array}$ & $<3$ & 30 & 9 & 2,000 \\
\hline & 3,600 & U & & 71 & 3,100 & 200 & 18 & 61 & $<30$ & $<1$ & & 5. & 30 & $<0.6$ & $<1$ & 4.6 & 14 & 7.3 & & & $<1$ & 22 & & $<3$ & 30 & 12 & 6 \\
\hline & 000 & 0 & 000 & 55 & 1,100 & 34 & 42 & 96 & \begin{tabular}{|l|}
34 \\
\end{tabular} & 2 & & $<$ & 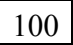 & 0. & 43 & 32 & $<0.6$ & 79 & 3.7 & & & 5.0 & 480 & $<3$ & 20 & 6.6 & 000 \\
\hline L- & 3,000 & 200 & & 33 & 360 & $<30$ & 25 & 880 & $<30$ & 17 & , & $<3$ & 100 & & 16 & 44 & $<0.6$ & 120 & & & & \begin{tabular}{|l|l|}
6.4 \\
\end{tabular} & 3,500 & $<3$ & 21 & 3.7 & 00 \\
\hline L- & 100 & 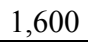 & & 31 & 46 & 30 & 26 & 83 & $<30$ & 15 & 1,6 & $<3$ & 170 & U. & 33 & 59 & $<0.6$ & 140 & 3.0 & 12,000 & 2 & 11 & 2,200 & $<3$ & 29 & 7 & 2,600 \\
\hline L-164 & 2,100 & $\underline{0}$ & & 28 & 61 & 46 & 33 & 840 & $<30$ & 5.0 & 600 & $<3$ & 52 & $<0.6$ & 6.1 & 6.8 & $<0.6$ & 74 & & 0 & 1 & 2 & 940 & $<3$ & 16 & 6 & 80 \\
\hline L-165 & 2,900 & 0 & 000 & 32 & 650 & 66 & 36 & 900 & $<30$ & 8.0 & 1,400 & $<3$ & 5 & $<0.6$ & \begin{tabular}{|l|}
9.1 \\
\end{tabular} & \begin{tabular}{|l|}
15 \\
\end{tabular} & $<0.6$ & 1 & 3.0 & 1,900 & 1 & 3 & 1,300 & $<3$ & 28 & 8 & 760 \\
\hline & 900 & 0 & 000 & 28 & 580 & 40 & 35 & 930 & $<30$ & 16 & 1,500 & $<3$ & 6 & $<0.6$ & 13 & \begin{tabular}{|l|}
8.6 \\
\end{tabular} & $<0.6$ & 200 & 2 & 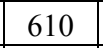 & 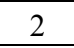 & 2 & 1,800 & $<3$ & 19 & 6.2 & 90 \\
\hline & 10 & & & 1900 & 2400 & 85 & 88 & 1,400 & 100 & 1 & & $<$ & & 1 & 24 & 45 & 1 & 40 & 6 & 0 & 6.2 & 6.5 & 420 & $<3$ & 38 & 22 & 700 \\
\hline & & & & 33 & 1,000 & 68 & 390 & 1,100 & $<30$ & 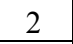 & & $<3$ & 110 & 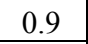 & 26 & 32 & $<0.6$ & 130 & 3.4 & 1,700 & 2 & \begin{tabular}{|l|} 
\\
\end{tabular} & 600 & $<3$ & 30 & 7.8 & 2,000 \\
\hline 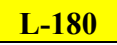 & & & & 82 & 1,300 & 82 & 1,300 & 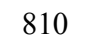 & 230 & $<1$ & $<r$ & $<3$ & 57 & $<0.6$ & $<1$ & 4.2 & 0 & 2 & 3 & 60 & $<1$ & 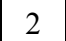 & 4 & $<3$ & 11 & 6 & 8 \\
\hline
\end{tabular}


Table 11. Streambed sediment data using the partial digestion method for the upper Prickly Pear Creek watershed, Montana, July 2001. (Continued)

\begin{tabular}{|c|c|c|c|c|c|c|c|c|c|c|c|c|c|c|c|c|c|c|c|c|c|c|c|c|c|c|c|}
\hline $\begin{array}{l}\text { Field } \\
\text { No. }\end{array}$ & Al & $\mathbf{C a}$ & $\mathrm{Fe}$ & $K$ & Mg & \begin{tabular}{|l|}
$\mathbf{N a}$ \\
\end{tabular} & $\mathbf{P}$ & $\mathbf{S i}$ & $\mathbf{T i}$ & Ag & As & B & $\mathbf{B a}$ & $\mathbf{B e}$ & Cd & Co & $\mathrm{Cr}$ & $\mathbf{C u}$ & $\mathbf{L i}$ & Mn & \begin{tabular}{|l|} 
Mo \\
\end{tabular} & $\mathbf{N i}$ & $\mathbf{P b}$ & \begin{tabular}{|l|} 
Sb \\
\end{tabular} & $\mathrm{Sr}$ & $\mathbf{V}$ & $\mathbf{Z n}$ \\
\hline PP-2a & 500 & 300 & 900 & 750 & 100 & \begin{tabular}{|l|}
63 \\
\end{tabular} & 100 & 960 & 70 & $<1$ & 7 & $<3$ & 37 & 0.6 & 1 & 3.5 & 4.3 & 18 & 4.0 & 380 & $<1$ & 4.1 & 52 & $<3$ & 20 & 12 & 160 \\
\hline PP-2b & 500 & 4,400 &, 000 & 1,000 & 300 & 64 & 680 & 1,500 & $<30$ & 16 & 160 & $<3$ & 140 & 0.6 & 19 & 5.4 & \begin{tabular}{|l|}
4.6 \\
\end{tabular} & 310 & 3.9 & 900 & $<1$ & 11 & 000 & $<3$ & 41 & 20 & 2,900 \\
\hline PP-3a & 2,700 & 4,100 & 7,500 & 980 & 000 & 80 & 1,600 & 1,200 & 230 & 2 & 36 & $<3$ & 60 & 0.6 & 3 & 5.5 & 3.2 & 53 & 4.1 & 1,200 & 2 & 3.8 & 350 & $<3$ & 27 & 17 & 500 \\
\hline PP-3b & 200 & 400 & 6,600 & 930 & 800 & \begin{tabular}{|l|}
59 \\
\end{tabular} & 1,300 & 990 & 210 & 3.8 & 31 & $<3$ & 57 & 0.6 & 4.0 & 3.9 & 2 & 48 & 3.8 & 700 & 1 & 3.4 & 400 & $<3$ & 18 & 13 & 700 \\
\hline PP-3r & 3,000 & 3,100 & 11,000 & 1,100 & 2,000 & 64 & 920 & 1,100 & 94 & 8.9 & 110 & $<3$ & 85 & $<0.6$ & 8.8 & 4.5 & 3.6 & 160 & 4.1 & 5,200 & 2 & 6.8 & 1,200 & $<3$ & 23 & 15 & 1,800 \\
\hline PP-4a & 1,900 & 3,600 & 7,100 & 760 & 1,500 & \begin{tabular}{|l|}
85 \\
\end{tabular} & 1,400 & 970 & 190 & 2 & 37 & $<3$ & 58 & $<0.6$ & 3.0 & 4.8 & 3 & 37 & 3.1 & 400 & $<1$ & 3.1 & 250 & $<3$ & 17 & 14 & 610 \\
\hline PP-4b & 1,800 & 3,300 & 5, & 670 & 00 & 10 & 1. & 980 & 170 & 1 & $\overline{19}$ & $<3$ & 53 & 0.6 & 2 & 3.6 & 2 & 2 & 3 & 00 & $<1$ & 2 & 110 & $<3$ & 15 & 12 & 420 \\
\hline PP-4r & 2,700 & 3,400 & 7,300 & 1,100 & 2,000 & \begin{tabular}{|l|}
68 \\
\end{tabular} & 1,100 & 1,100 & 280 & 3.0 & 33 & $<3$ & 79 & $<0.6$ & 4.2 & 5.0 & 2 & 55 & 5.0 & 2,000 & $<1$ & 4.3 & 340 & $<3$ & 23 & 13 & 880 \\
\hline PP-5r & 200 & 3,800 & 6,800 & 910 & 1,600 & \begin{tabular}{|l|}
73 \\
\end{tabular} & 1,400 & 880 & 240 & 1 & 20 & $<3$ & 63 & $<0.6$ & 2 & 3.5 & 3.6 & 23 & 4.2 & 900 & $<1$ & 5.2 & 140 & $<3$ & 15 & 14 & 450 \\
\hline PP-100 & 3,100 & 5,700 & 8,400 & 1,500 & 2,100 & 63 & 1,100 & 1,000 & 200 & $<1$ & 9 & 3.4 & 140 & $<0.6$ & 2 & 6.0 & 3.2 & 45 & 4.8 & 350 & $<1$ & 5.0 & 44 & \begin{tabular}{|l|}
$<3$ \\
\end{tabular} & 37 & 14 & 98 \\
\hline PP-200 & 2,500 & 11,000 & 6,200 & 960 & 4,200 & 100 & 1,600 & 980 & 230 & $<1$ & 22 & $<3$ & 62 & $<0.6$ & 1 & 3.8 & 3.0 & 19 & 4.6 & 960 & $<1$ & 3.9 & 110 & $<3$ & 21 & 12 & 430 \\
\hline PP-201 & 2,400 & 0 & & 1,000 & 00 & 86 & 1,200 & 0 & 270 & 1 & 15 & $<3$ & 59 & 0.6 & 2 & 4.9 & 3 & 24 & 4.4 & 000 & $<1$ & & 120 & $<3$ & 21 & 12 & 460 \\
\hline S-3r & 2,100 & 2,500 & 8,000 & 720 & 1,400 & 87 & 800 & 1,000 & 66 & 2 & 150 & $<3$ & 55 & $<0.6$ & 7.6 & 5.4 & 3 & 73 & 2 & 960 & $<1$ & 6.1 & 500 & $<3$ & 24 & 10 & 630 \\
\hline S-4r & 2,400 & 4,600 & 16,000 & 700 & 1,500 & 57 & 820 & 1,600 & $<30$ & 27 & 310 & $<3$ & 180 & 0.6 & 31 & $<0.6$ & 1 & 430 & 2 & 17,000 & 3 & 8.8 & 3,900 & \begin{tabular}{|l|}
8.2 \\
\end{tabular} & 46 & 8.7 & 5,400 \\
\hline $\mathrm{S}-200$ & 5,300 & 4,800 & 14,000 & 2,800 & 4,000 & 56 & 1,000 & 1,200 & 480 & 2 & 21 & $<3$ & 140 & $<0.6$ & 3.2 & 14 & 4.5 & 74 & 9.3 & 1,100 & $<1$ & 8.6 & 210 & $<3$ & 23 & 28 & 360 \\
\hline S-201 & 5,000 & 3,400 & 11,000 & 2,800 & 4,100 & 87 & 1,100 & 1,500 & 640 & $<1$ & 14 & $<3$ & 110 & $<0.6$ & 1 & 11 & \begin{tabular}{|l|}
4.1 \\
\end{tabular} & 96 & 8.9 & 570 & $<1$ & 6.3 & 60 & $<3$ & 15 & 24 & 170 \\
\hline S-202 & 3,500 & 2,900 & 4,400 & 1,400 & 1,200 & 68 & 460 & 880 & 150 & $<1$ & 25 & $<3$ & 89 & $<0.6$ & 2 & 3.8 & 4.3 & 37 & 2 & 380 & $<1$ & 3.3 & 170 & $<3$ & 38 & 5.4 & 150 \\
\hline S-203 & 2,200 & 440 & 15,000 & 530 & 650 & 60 & 500 & 780 & $<30$ & $<1$ & 1 & $<3$ & 56 & $<0.6$ & $<1$ & 4.3 & 0.8 & 93 & 1 & 94 & 23 & 2 & 45 & $<3$ & 8.3 & 9.5 & 39 \\
\hline S-204 & 1,400 & 2,200 & 30,000 & 560 & 900 & 59 & 370 & 750 & $<30$ & 9.9 & 4,100 & 3.8 & 57 & $<0.6$ & 8.5 & 11 & 2 & 80 & 1 & 1,800 & 1 & 4.0 & 1,600 & $<3$ & 19 & 5.8 & 1,500 \\
\hline S-205 & 4,400 & 3,500 & 11,000 & 1,500 & 3,800 & 110 & 780 & 1,300 & 140 & 2 & 36 & $<3$ & 120 & $<0.6$ & 7.5 & 11 & 5.4 & 94 & 4.8 & 2,300 & 1 & 8.9 & 360 & $<3$ & 42 & 17 & 950 \\
\hline S-206 & 5,000 & 5,000 & 12,000 & 1,600 & 3,600 & 120 & 810 & 1,100 & 160 & 3.0 & 48 & $<3$ & 130 & $<0.6$ & 8.7 & 10 & \begin{tabular}{|l|}
14 \\
\end{tabular} & 120 & 5.4 & 2,600 & $<1$ & 13 & 410 & $<3$ & 45 & 18 & 1,200 \\
\hline WS-8 & 2,400 & 4,000 & 5,800 & 1,000 & 2,000 & 110 & 1,400 & 1,000 & 310 & $<1$ & 27 & $<3$ & 50 & 0.7 & 1 & 4.7 & 2 & 9.8 & 4.8 & 200 & $<1$ & 3 & 24 & $<3$ & 25 & 12 & 200 \\
\hline
\end{tabular}




\title{
BIOLOGICAL DATA FOR STREAMS IN THE UPPER PRICKLY PEAR CREEK WATERSHED, MONTANA, 2001
}

\author{
By Aïda M. Farag and David D. Harper
}

\section{INTRODUCTION}

This section presents biological data that were collected in the upper Prickly Pear watershed during August 2001. Data presented in this section include measurements of metal residues in biofilm (aufwuchs), benthic macroinvertebrates, and fish tissues, histological analysis, results from lipid peroxidation bio-assays, metallothionein tissue concentrations, and biomass estimates.

Site numbers for biological sampling were similar to the alpha-numeric system used for water quality and sediment sampling efforts. The alpha character denotes the first letter of the stream name and the numeric characters denote the downstream sequence, beginning at the most upstream site (fig.1). Because multiple biological samples were collected from identical sites, additional numeric designations refer to the individual samples.

\section{BIOLOGICAL DATA}

Biological samples were collected from 14 sites (fig.1). Biofilm was collected from 14 sites, benthic macroinvertebrates were collected from 6 sites, and fish tissues were collected from 5 sites. As, $\mathrm{Cd}, \mathrm{Cu}, \mathrm{Pb}$, and $\mathrm{Zn}$ were measured in all samples and reported as $\mu \mathrm{g} / \mathrm{g}$ dry weight (table 12). Metal residue data for aquatic vegetation, biofilm, benthic macroinvertebrates, individual fish tissues; results of histological analysis; lipid peroxidation data, metallothionein data, and trout population and biomass assessment data are also reported in table 12 .

\section{Methods}

Sampling methods were similar to those described by Farag et al. (1998). Rocks along the shore were removed from the water and the rock surface was scraped gently with acid-washed plastic utensils. The biofilm was placed directly in acid-washed plastic vials. For benthic macroinvertebrates, an area of approximately $6 \mathrm{~m}^{2}$ was overturned upstream of a 3-mm mesh net. Benthic macroinvertebrates were removed with plastic or stainless steel forceps and placed in acid-washed vials. The benthic macroinvertebrates were rinsed with stream water to remove debris, but they were not allowed time to depurate (purge the contents of the gut). Without depuration, the metal concentrations in the invertebrates represent the "dose" of metals received by fish. Fish were collected by electrofishing; fish were pithed and lengths and weights were recorded. Individual fish were placed in plastic bags for whole fish analyses of metals. Fillet, gills, livers, and pyloric caeca were removed from additional fish and each tissue type was placed in an acid-washed plastic vial. In the laboratory, the fillet, gills, livers, and pyloric caeca of approximately three fish from each sample site were combined for a composite sample of each tissue type. The chemical element content of all samples was measured by atomic absorption spectroscopy with a graphite furnace or flame source. The tissues were dried at $58^{\circ} \mathrm{C}$ for 48 hours and digested with $30 \%$ nitric acid at $76^{\circ} \mathrm{C}$ for 48 hours. Quality control was monitored for all chemical analyses. Instrument calibration was verified by analyzing certified calibration solutions during each instrumental run. These external 
reference standards were generally within $80 \%$ to $120 \%$ of the nominal concentrations. All of the sample spikes for tissues were within $80 \%$ to $120 \%$ recovery, except when the tissues were heterogeneous (biofilm and pyloric caeca). Digestion blanks were analyzed to detect potential contamination during the digestion procedure. In all cases, the average concentration of the digestion blanks was less than 2 times the instrument detection limit; therefore, we did not adjust the tissue concentrations.

Histological samples were collected, preserved in Davidson's fixative, and transferred to alcohol. Tissues were dehydrated and embedded in paraffin, sectioned to 5 $\mu \mathrm{m}$, stained with hemotoxylion \& eosin and examined by light microscopy. Slides were examined with no knowledge of site or species. Cellular and tissue alterations were graded on a scale of $0-5 ; 0=$ none, $1=$ minimal, $=2=$ mild, $3=$ moderate, $4=$ moderately severe, $5=$ severe.

The products of lipid peroxidation were measured using a fluorometric assay to measure the relative intensity of fluorophores formed during lipid peroxidation (Dillard and Tappel 1984; Fletcher and others, 1973; Farag and others, 1995). Frozen tissue was combined with chloroform, methanol and water, homogenized, vortexed and centrifuged. After removing the chloroform layer, fluorescence was measured at a wavelength of 435 $\mathrm{nm}$ emission during excitation at 340 and $360 \mathrm{~nm}$.

Metallothionein concentrations were determined using a double-antibody radioammunoassay developed for fish tissues (Hogstrand et. al. 1994). Trout population and biomass data were collected and analyzed using using the three pass removal method (Zippen 1958). 
Table 12. Biological data for streams in the upper Prickly Pear Creek watershed, Montana, 2001. (Includes data for biofilm, benthic macroinvertebrates; individual fish tissues; results of histological analysis; lipid peroxidation data; metallothionein data and trout population and biomass data)

[All measurements are as $\mu \mathrm{g} / \mathrm{g}$ dry weight. $\mathrm{BIO}=$ biofilm, $\mathrm{FIL}=$ filet, $\mathrm{INV}=$ benthic macroinvertebrates, $\mathrm{CAECA}=$ pyloric caeca, $\mathrm{WF}=$ Whole Fish, WSC $=$ Westslope Cutthroat, GI=gastrointestinal, N/A= no data or not analyzed (in lipid peroxidation part of table), $=$ not computed (histological analysis). $<=$ less than the value listed to right. Cellular and tissue alterations were graded on a scale of 0-5; $0=$ none, $1=$ minimal, $2=$ mild, $3=$ moderate, $4=$ moderately severe, $5=$ severe. Lipid peroxidation measured at wavelengths of 340 and $360 \mu \mathrm{m}$.]

\section{Biofilm, and Benthic Macroinvertebrates}

\begin{tabular}{|c|c|c|c|c|c|c|c|c|}
\hline Site ID & Date & Sample ID & Type & $\mu \mathrm{g}$ As/g & $\mu \mathrm{g} \mathrm{Cd} / \mathrm{g}$ & $\mu \mathrm{g} \mathrm{Cu} / \mathrm{g}$ & $\mu \mathrm{g} \mathrm{Pb} / \mathrm{g}$ & $\mu \mathrm{g} \mathrm{Zn} / \mathrm{g}$ \\
\hline PP-1 & $8 / 24 / 01$ & PP1-INV-1 & INV & 1.14 & 2.00 & 32.1 & 0.37 & 229 \\
\hline PP-1 & $8 / 24 / 01$ & PP1-INV-2 & INV & 1.63 & 2.55 & 28.1 & 1.46 & 254 \\
\hline PP-1 & $8 / 24 / 01$ & PP1-INV-3 & INV & 1.28 & 2.37 & 26.9 & 0.71 & 262 \\
\hline PP-1 & 8/24/01 & PP1-INV-4 & INV & 1.31 & 2.36 & 27.6 & 1.680 & 259 \\
\hline PP-1 & $8 / 24 / 01$ & PP1-BIO-1 & $\mathrm{BIO}$ & 8.50 & 0.76 & 20.7 & 10.6 & 111 \\
\hline PP-1 & $8 / 24 / 01$ & PP1-BIO-2 & $\mathrm{BIO}$ & 7.08 & 0.48 & 19.1 & 9.78 & 96 \\
\hline PP-1 & $8 / 24 / 01$ & PP1-BIO-3 & $\mathrm{BIO}$ & 5.45 & 0.36 & 11.0 & 3.89 & 66 \\
\hline PP-1 & 8/24/01 & PP1-BIO-4 & $\mathrm{BIO}$ & 8.32 & 0.86 & 20.8 & 6.01 & 111 \\
\hline PP-3 & $8 / 24 / 01$ & PP3-INV-1 & INV & 17.0 & 5.43 & 79.7 & 121 & 1,340 \\
\hline PP-3 & $8 / 24 / 01$ & PP3-INV-2 & INV & 6.92 & 2.62 & 52.7 & 67.9 & 785 \\
\hline PP-3 & $8 / 24 / 01$ & PP3-INV-3 & INV & 15.6 & 4.43 & 51.1 & 85.3 & 1050 \\
\hline PP-3 & 8/24/01 & PP3-INV-4 & INV & 19.8 & 4.81 & 80.8 & 126 & 970 \\
\hline PP-3 & $8 / 24 / 01$ & PP3-BIO-1 & $\mathrm{BIO}$ & 257 & 274 & 1,030 & 1880 & 50,100 \\
\hline PP-3 & 8/24/01 & PP3-BIO-2 & $\mathrm{BIO}$ & 275 & 477 & 1,754 & 1740 & 87,700 \\
\hline PP-3 & 8/24/01 & PP3-BIO-3 & $\mathrm{BIO}$ & 267 & 478 & 1,584 & 1630 & 96,400 \\
\hline PP-3 & 8/24/01 & PP3-BIO-4 & $\mathrm{BIO}$ & 316 & 489 & 1,610 & 2020 & 99,700 \\
\hline PP-4 & $8 / 27 / 01$ & PP4-BIO-1 & $\mathrm{BIO}$ & 64 & 20.3 & 126 & 640 & 4,400 \\
\hline PP-4 & $8 / 27 / 01$ & PP4-BIO-2 & $\mathrm{BIO}$ & 85.5 & 20.4 & 130 & 589 & 4,550 \\
\hline PP-4 & $8 / 27 / 01$ & PP4-BIO-3 & $\mathrm{BIO}$ & 64.77 & 20.1 & 134 & 652 & 4,600 \\
\hline PP-4 & $8 / 27 / 01$ & PP4-BIO-4 & $\mathrm{BIO}$ & 449 & 10.6 & 90.2 & 446 & 2,8002 \\
\hline PP-5 & $8 / 24 / 01$ & PP5-INV-1 & INV & 7.42 & 2.30 & 58.3 & 19.9 & 761 \\
\hline PP-5 & 8/24/01 & PP5-INV-2 & INV & 10.6 & 2.30 & 47.2 & 36.8 & 762 \\
\hline PP-5 & $8 / 24 / 01$ & PP5-INV-3 & INV & 8.55 & 1.94 & 51.3 & 23.0 & 673 \\
\hline PP-5 & 8/24/01 & PP5-INV-4 & INV & 9.22 & 2.42 & 58.3 & 32.1 & 792 \\
\hline PP-5 & $8 / 24 / 01$ & PP5-BIO-1 & $\mathrm{BIO}$ & 110 & 22.9 & 96.4 & 356 & 5,360 \\
\hline PP-5 & $8 / 24 / 01$ & PP5-BIO-2 & $\mathrm{BIO}$ & 66.8 & 10.3 & 88.6 & 359 & 2,310 \\
\hline PP-5 & 8/24/01 & PP5-BIO-3 & $\mathrm{BIO}$ & 64.5 & 11.8 & 88.8 & 383 & 2,690 \\
\hline PP-5 & $8 / 24 / 01$ & PP5-BIO-4 & $\mathrm{BIO}$ & 60.4 & 7.80 & 75.7 & 352 & 1,820 \\
\hline C-5 & $8 / 27 / 01$ & C5-BIO-1 & $\mathrm{BIO}$ & 162 & 17.4 & 69.7 & 96.2 & 2,270 \\
\hline$C-5$ & 8/27/01 & C5-BIO-2 & $\mathrm{BIO}$ & 134 & 11.3 & 55.1 & 138.6 & 1,420 \\
\hline$C-5$ & $8 / 27 / 01$ & C5-BIO-3 & $\mathrm{BIO}$ & 278 & 47.7 & 239 & 84.8 & 17,800 \\
\hline $\mathrm{C}-5$ & $8 / 27 / 01$ & C5-BIO-4 & $\mathrm{BIO}$ & 158 & 15.6 & 63.1 & 44.3 & 6,070 \\
\hline L-1a & $8 / 23 / 01$ & L1A-INV-1 & INV & 3.87 & 4.17 & 29.9 & 6.98 & 907 \\
\hline L-1a & 8/23/01 & L1A-INV-2 & INV & 9.73 & 7.40 & 33.5 & 9.81 & 1,140 \\
\hline L-1a & 8/23/01 & L1A-INV-3 & INV & 10.0 & 3.95 & 24.1 & 2.60 & 793 \\
\hline L-1a & 8/23/01 & L1A-INV-4 & INV & 7.22 & 4.26 & 27.1 & 5.57 & 1,040 \\
\hline L-1a & $8 / 23 / 01$ & L1A-BIO-1 & $\mathrm{BIO}$ & 141 & 20.7 & 55.9 & 32.5 & 1,860 \\
\hline L-1a & 8/23/01 & L1A-BIO-2 & $\mathrm{BIO}$ & 28.0 & 1.27 & 17.7 & 21.5 & 184 \\
\hline L-1a & 8/23/01 & L1A-BIO-3 & $\mathrm{BIO}$ & 38.8 & 3.12 & 24.4 & 15.2 & 410 \\
\hline L-1a & 8/23/01 & L1A-BIO-4 & $\mathrm{BIO}$ & 26.4 & 1.63 & 23.5 & 27.9 & 234 \\
\hline$L-3 b$ & $8 / 23 / 01$ & L3-B & $\mathrm{BIO}$ & 1940 & 463 & 226 & 619 & 49,800 \\
\hline$L-3 b$ & 8/23/01 & L3-B & $\mathrm{BIO}$ & 1490 & 586 & 245 & 547 & 61,600 \\
\hline$L-3 b$ & 8/23/01 & L3-B & $\mathrm{BIO}$ & 1,110 & 1,018 & 330 & 371 & 87,200 \\
\hline$L-3 b$ & 8/23/01 & L3-B & $\mathrm{BIO}$ & 1,460 & 870 & 355 & 732 & 79,300 \\
\hline L-5 & $8 / 27 / 01$ & L5-BIO-1 & $\mathrm{BIO}$ & 0.89 & 15.6 & 42.5 & 36.4 & 4,120 \\
\hline$L-5$ & $8 / 27 / 01$ & L5-BIO-2 & $\mathrm{BIO}$ & 4.40 & 4.9 & 19.4 & 10.8 & 1,800 \\
\hline$L-5$ & $8 / 27 / 01$ & L5-BIO-3 & $\mathrm{BIO}$ & 1.38 & 11.3 & 22.5 & 7.19 & 2,790 \\
\hline L-5 & 8/27/01 & L5-BIO-4 & $\mathrm{BIO}$ & 4.31 & 13.1 & 35.8 & 31.1 & 3,550 \\
\hline
\end{tabular}


Biofilm, and Benthic Macroinvertebrates (Continued)

\begin{tabular}{|c|c|c|c|c|c|c|c|c|}
\hline Site ID & Date & Sample ID & Type & $\mu \mathrm{g}$ As $/ \mathrm{g}$ & $\mu \mathrm{g} \mathrm{Cd} / \mathrm{g}$ & $\mu \mathrm{g} \mathrm{Cu} / \mathrm{g}$ & $\mu \mathrm{g} \mathrm{Pb} / \mathrm{g}$ & $\mu \mathrm{g} \quad \mathrm{Zn} / \mathrm{g}$ \\
\hline$\overline{\mathrm{LL}-7}$ & $8 / 27 / 01$ & L7-BIO-1 & $\mathrm{BIO}$ & 8.52 & 0.62 & 78.8 & 111.4 & 123 \\
\hline L-7 & $8 / 27 / 01$ & L7-BIO-2 & $\mathrm{BIO}$ & 12.9 & 10.8 & 158.5 & 2.89 & 1,350 \\
\hline L-7 & $8 / 27 / 01$ & L7-BIO-3 & $\mathrm{BIO}$ & 9.90 & 0.59 & 69.1 & 4.71 & 104 \\
\hline $\mathrm{L}-7$ & $8 / 27 / 01$ & L7-BIO-4 & $\mathrm{BIO}$ & 8.40 & 0.55 & 70.2 & 7.92 & 111 \\
\hline 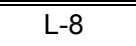 & $8 / 27 / 01$ & L8-BIO-1 & $\mathrm{BIO}$ & 19.3 & 3.01 & 19.7 & 15.3 & 969 \\
\hline L-8 & $8 / 27 / 01$ & L8-BIO-2 & $\mathrm{BIO}$ & 26.6 & 8.40 & 33.6 & 22.0 & 1,970 \\
\hline L-8 & $8 / 27 / 01$ & L8-BIO-3 & $\mathrm{BIO}$ & 21.5 & 5.62 & 29.5 & 18.0 & 1,670 \\
\hline L-8 & $8 / 27 / 01$ & L8-BIO-4 & $\mathrm{BIO}$ & 25.0 & 7.65 & 41.3 & 25.6 & 2,320 \\
\hline UULGP & $8 / 8 / 26 / 01$ & ULG-BIO-1 & $\mathrm{BIO}$ & 19.3 & 8.71 & 19.1 & 38.0 & 2,030 \\
\hline ULGP & $8 / 26 / 01$ & ULG-BIO-2 & $\mathrm{BIO}$ & 39.6 & 21.6 & 38.8 & 93.8 & 3,385 \\
\hline ULGP & 8/26/01 & ULG-BIO-3 & $\mathrm{BIO}$ & 17.0 & 7.67 & 19.0 & 36.1 & 1,680 \\
\hline ULGP & 8/26/01 & ULG-BIO-4 & $\mathrm{BIO}$ & 55.9 & 23.4 & 40.6 & 61.2 & 4,530 \\
\hline 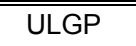 & $8 / 26 / 01$ & ULLG-INV-1 & IINV & 6.83 & 4.17 & 29.9 & 6.98 & 907 \\
\hline ULGP & 8/26/01 & ULG-INV-2 & INV & 12.7 & 7.40 & 33.5 & 9.81 & 1140 \\
\hline ULGP & 8/26/01 & ULG-INV-3 & INV & 5.57 & 3.95 & 24.1 & 2.60 & 793 \\
\hline ULGP & 8/26/01 & ULG-INV-4 & INV & 8.02 & 4.26 & 27.2 & 5.57 & 1040 \\
\hline WS-7 & $8 / 8 / 27 / 01$ & WS7-BIO-1 & $\mathrm{BIO}$ & "64.0 & 6.46 & 13.8 & 8.22 & 1,210 \\
\hline WS-7 & $8 / 27 / 01$ & WS7-BIO-2 & $\mathrm{BIO}$ & 85.5 & 7.06 & 18.2 & 13.1 & 1,300 \\
\hline WS-7 & $8 / 27 / 01$ & WS7-BIO-3 & $\mathrm{BIO}$ & 64.7 & 1.29 & 12.4 & 25.2 & 366 \\
\hline WS-7 & $8 / 27 / 01$ & WS7-BIO-4 & $\mathrm{BIO}$ & 449 & 4.38 & 30.1 & 60.0 & 1,130 \\
\hline WS-2 & $8 / 27 / 01$ & WS2-BIO-1 & $\mathrm{BIO}$ & 1,130 & 126 & 186 & 666 & 13,500 \\
\hline WS-2 & $8 / 27 / 01$ & WS2-BIO-2 & $\mathrm{BIO}$ & 1,180 & 101 & 88.4 & 176 & 14,600 \\
\hline WS-2 & $8 / 27 / 01$ & WS2-BIO-3 & $\mathrm{BIO}$ & 564 & 67.7 & 133 & 96.1 & 4,750 \\
\hline WS-2 & $8 / 27 / 01$ & WS2-BIO-4 & $\mathrm{BIO}$ & 1,260 & 25.7 & 93.8 & 203 & 4,620 \\
\hline WS-3 & $8 / 27 / 01$ & "WS3-INV-1 & IINV & 46.0 & 5.95 & 488.2 & 17.8 & 788 \\
\hline WS-3 & $8 / 27 / 01$ & WS3-INV-2 & INV & 29.7 & 4.95 & 43.3 & 16.2 & 1,130 \\
\hline WS-3 & $8 / 27 / 01$ & WS3-INV-3 & INV & 34.5 & 4.41 & 42.9 & 13.0 & 842 \\
\hline WS-3 & $8 / 27 / 01$ & WS3-INV-4 & INV & 30.9 & 3.53 & 37.3 & 12.7 & 766 \\
\hline WS-3 & $8 / 27 / 01$ & WS3-BIO-1 & $\mathrm{BIO}$ & 1,440 & 13.6 & 106 & 660 & 6,640 \\
\hline WS-3 & 8/27/01 & WS3-BIO-2 & $\mathrm{BIO}$ & 1,300 & 18.7 & 87.5 & 553 & 5,800 \\
\hline WS-3 & $8 / 27 / 01$ & WS3-BIO-3 & $\mathrm{BIO}$ & 927 & 27.2 & 71.5 & 396 & 5,590 \\
\hline WS-3 & $8 / 27 / 01$ & WS3-BIO-4 & $\mathrm{BIO}$ & 248 & 58.6 & 102 & 106 & 3,870 \\
\hline
\end{tabular}


Individual fish tissues

\begin{tabular}{|c|c|c|c|c|c|c|c|c|c|c|c|c|}
\hline Site ID & Date & Species & Sex & $L(\mathrm{~mm})$ & wt (gm) & Type & Sample Id & $\mu \mathrm{g}$ As/g & $\mu \mathrm{g} \mathrm{Cd} / \mathrm{g}$ & $\mu \mathrm{g} \mathrm{Cu} / \mathrm{g}$ & $\mu \mathrm{g} \mathrm{Pb} / \mathrm{g}$ & $\mu \mathrm{g} \mathrm{Zn} / \mathrm{g}$ \\
\hline PP-1 & $8 / 29 / 01$ & BROOK & $\bar{F}$ & 185 & 82 & \multirow{2}{*}{$\begin{array}{l}\text { LIVER } \\
\text { LIVER }\end{array}$} & \multirow[t]{2}{*}{ "MT-PP1-LIV1 } & \multirow[t]{2}{*}{0.24} & \multirow[t]{2}{*}{2.95} & \multirow[t]{2}{*}{ "58.1 } & \multirow[t]{2}{*}{ 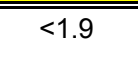 } & \multirow[t]{2}{*}{1110.0} \\
\hline PP-1 & $8 / 29 / 01$ & BROOK & M & 222 & 130 & & & & & & & \\
\hline PP-1 & $8 / 29 / 01$ & BROOK & $\mathrm{F}$ & 200 & 82 & LIVER & \multirow[t]{2}{*}{ MT-PP1-LIV2 } & \multirow[t]{2}{*}{0.19} & \multirow[t]{2}{*}{2.31} & \multirow[t]{2}{*}{27.6} & \multirow[t]{2}{*}{$<2.0$} & \multirow[t]{2}{*}{128} \\
\hline PP-1 & $8 / 29 / 01$ & BROOK & $M$ & 180 & 63 & LIVER & & & & & & \\
\hline PP-1 & $8 / 29 / 01$ & BROOK & $\mathrm{F}$ & 190 & 70 & LIVER & \multirow[t]{2}{*}{ MT-PP1-LIV3 } & \multirow[t]{2}{*}{0.22} & \multirow[t]{2}{*}{2.21} & \multirow[t]{2}{*}{24.6} & \multirow[t]{2}{*}{$<1.9$} & \multirow[t]{2}{*}{115} \\
\hline PP-1 & $8 / 29 / 01$ & BROOK & $M$ & 188 & 86 & LIVER & & & & & & \\
\hline PP-1 & $8 / 29 / 01$ & BROOK & $M$ & 168 & 60 & LIVER & \multirow{3}{*}{ MT-PP1-LIV4 } & \multirow{3}{*}{0.20} & \multirow{3}{*}{2.45} & \multirow{3}{*}{32.9} & \multirow{3}{*}{$<1.9$} & \multirow{3}{*}{98.8} \\
\hline PP-1 & $8 / 29 / 01$ & BROOK & $\mathrm{F}$ & 206 & 93 & LIVER & & & & & & \\
\hline PP-1 & $8 / 29 / 01$ & BROOK & M & 174 & 51 & LIVER & & & & & & \\
\hline PP-1 & $8 / 29 / 01$ & BROOK & $M$ & 194 & 84 & LIVER & MT-PP1-LIV5 & 0.34 & 1.52 & 38.1 & $<1.9$ & 102 \\
\hline PP-1 & $8 / 29 / 01$ & BROOK & $\mathrm{F}$ & 199 & 77 & LIVER & & & & & & \\
\hline PP-1 & $8 / 29 / 01$ & BROOK & $\mathrm{F}$ & 176 & 60 & LIVER & MT-PP1-LIV6 & 0.37 & 1.88 & 21.0 & $<1.9$ & 90.9 \\
\hline PP-1 & $8 / 29 / 01$ & BROOK & - & 205 & 95 & LIVER & & & & & & \\
\hline PP-1 & $8 / 29 / 01$ & BROOK & $M$ & 200 & 103 & LIVER & MT-PP1-LIV7 & 0.19 & 4.88 & 66.6 & $<1.9$ & 124 \\
\hline PP-1 & $8 / 29 / 01$ & BROOK & M & 210 & 121 & LIVER & & & & & & \\
\hline PP-1 & 8/29/01 & BROOK & 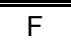 & 185 & 82 & 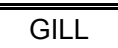 & "MT-PP1-GIL1 & 0.25 & 1.57 & 2.56 & 0.87 & 108 \\
\hline PP-1 & $8 / 29 / 01$ & BROOK & M & 222 & 130 & GILL & & & & & & \\
\hline PP-1 & $8 / 29 / 01$ & BROOK & $F$ & 200 & 82 & GILL & MT-PP1-GIL2 & 0.15 & 1.86 & 3.18 & $<0.79$ & 111 \\
\hline PP-1 & $8 / 29 / 01$ & BROOK & M & 180 & 63 & GILL & & & & & & \\
\hline PP-1 & $8 / 29 / 01$ & BROOK & $\mathrm{F}$ & 190 & 70 & GILL & & & & & & \\
\hline PP-1 & $8 / 29 / 01$ & BROOK & M & 188 & 86 & GILL & MT-PP1-GIL3 & 0.20 & 1.54 & 2.48 & 1.20 & 106 \\
\hline PP-1 & $8 / 29 / 01$ & BROOK & M & 168 & 60 & GILL & & & & & & \\
\hline PP-1 & $8 / 29 / 01$ & BROOK & $\mathrm{F}$ & 206 & 93 & GILL & MT-PP1-GIL4 & 0.16 & 1.43 & 3.37 & $<0.80$ & 111 \\
\hline PP-1 & $8 / 29 / 01$ & BROOK & M & 174 & 51 & GILL & & & & & & \\
\hline PP-1 & $8 / 29 / 01$ & BROOK & $M$ & 194 & 84 & GILL & MT-PP1-GIL5 & 0.40 & 1.14 & 3.10 & $<0.82$ & 103 \\
\hline PP-1 & 8/29/01 & BROOK & $\mathrm{F}$ & 199 & 77 & GILL & & & & & & \\
\hline PP-1 & $8 / 29 / 01$ & BROOK & $\mathrm{F}$ & 176 & 60 & GILL & MT-PP1-GIL6 & 0.34 & 1.22 & 2.52 & $<0.82$ & 98.9 \\
\hline PP-1 & $8 / 29 / 01$ & BROOK & - & 205 & 95 & GILL & & & & & & \\
\hline PP-1 & $8 / 29 / 01$ & BROOK & $M$ & 200 & 103 & GILL & MT-PP1-GIL7 & 0.21 & 1.59 & 3.02 & $<0.78$ & 118 \\
\hline PP-1 & $8 / 29 / 01$ & BROOK & M & 210 & 121 & GILL & & & & & & \\
\hline PP-1 & 8/29/01 & "BROOK & $\overline{F F}$ & 185 & 82 & CAECA & MT-PP1-PYC1 & 0.90 & 0.72 & 1.84 & $<<1.16$ & 134 \\
\hline PP-1 & $8 / 29 / 01$ & BROOK & M & 222 & 130 & CAECA & & & & & & \\
\hline PP-1 & $8 / 29 / 01$ & BROOK & $\mathrm{F}$ & 200 & 82 & CAECA & MT-PP1-PYC2 & 0.39 & 1.00 & 3.76 & $<1.39$ & 293 \\
\hline PP-1 & $8 / 29 / 01$ & BROOK & $M$ & 180 & 63 & CAECA & & & & & & \\
\hline
\end{tabular}


Individual fish tissues (Continued)

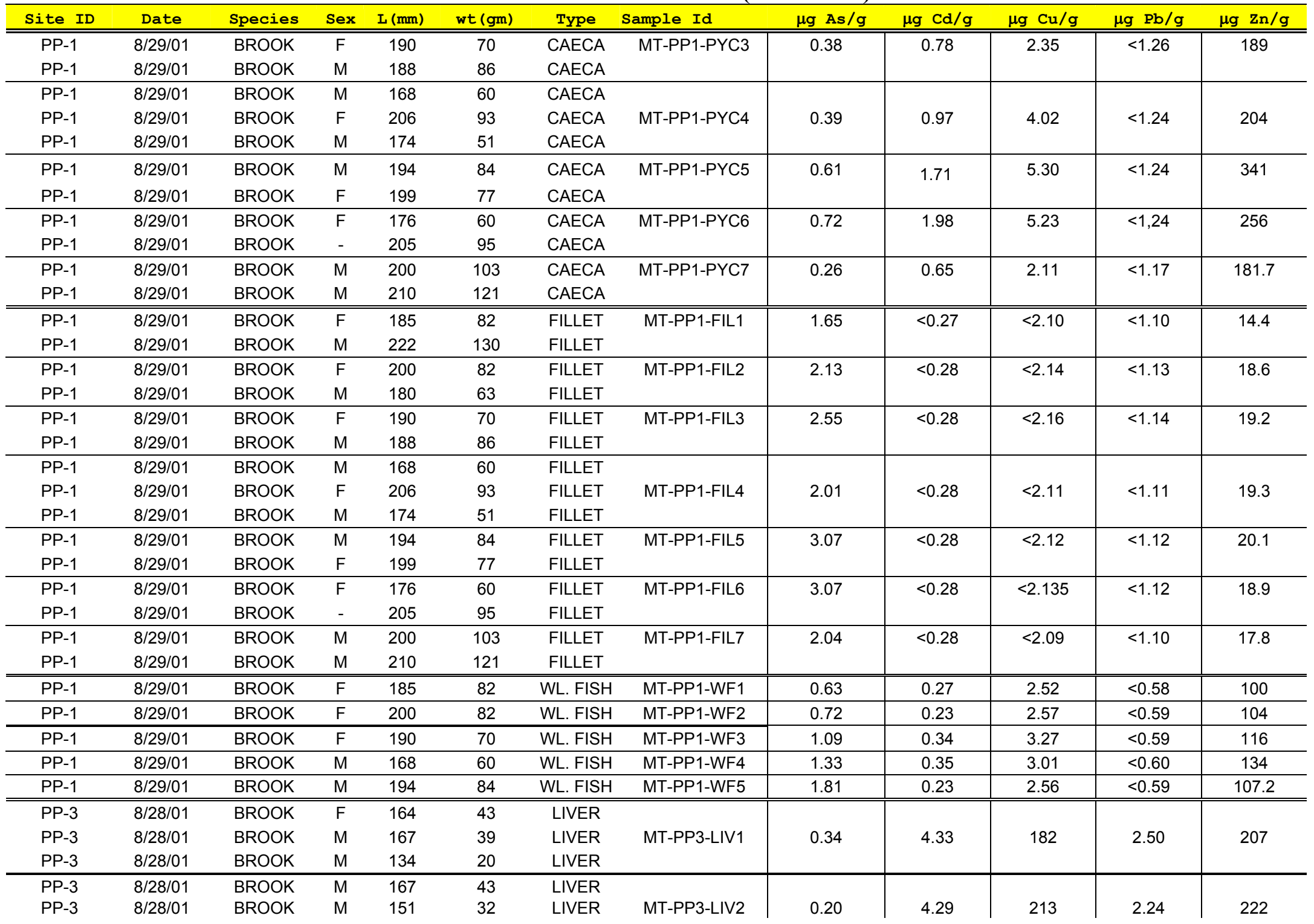


Individual fish tissues (Continued)

\begin{tabular}{|c|c|c|c|c|c|c|c|c|c|c|c|c|}
\hline Site ID & Date & Species & Sex & $\mathrm{L}(\mathrm{mm})$ & wt (gm) & Type & Sample Id & $\mu \mathrm{g} \mathrm{As/g}$ & $\mu \mathrm{g} \mathrm{Cd} / \mathrm{g}$ & $\mu \mathrm{g} \mathrm{Cu} / \mathrm{g}$ & $\mu \mathrm{g} \mathrm{Pb} / \mathrm{g}$ & $\mu \mathrm{g} \mathrm{Zn} / \mathrm{g}$ \\
\hline PP-3 & $8 / 28 / 01$ & BROOK & $\mathrm{F}$ & 139 & 28 & LIVER & & & & & & \\
\hline PP-3 & $8 / 28 / 01$ & BROOK & $\mathrm{F}$ & 140 & 22 & LIVER & & & & & & \\
\hline PP-3 & 8/28/01 & BROOK & M & 163 & 39 & LIVER & MT-PP3-LIV3 & 0.45 & 7.04 & 345 & 4.26 & 307 \\
\hline PP-3 & $8 / 28 / 01$ & BROOK & M & 169 & 46 & LIVER & & & & & & \\
\hline PP-3 & 8/28/01 & BROOK & M & 190 & 76 & LIVER & MT-PP3-LIV4 & 0.24 & 7.18 & 252 & 5.34 & 231 \\
\hline PP-3 & $8 / 28 / 01$ & BROOK & M & 150 & 32 & LIVER & & & & & & \\
\hline PP-3 & 8/28/01 & BROOK & $\mathrm{F}$ & 153 & 43 & LIVER & & & & & & \\
\hline PP-3 & $8 / 28 / 01$ & BROOK & $\mathrm{F}$ & 147 & 28 & LIVER & MT-PP3-LIV5 & 0.32 & 6.12 & 199 & 3.24 & 200 \\
\hline PP-3 & $8 / 28 / 01$ & BROOK & M & 151 & 29 & LIVER & & & & & & \\
\hline PP-3 & $8 / 28 / 01$ & BROOK & $\mathrm{F}$ & 243 & 140 & LIVER & MT-PP3-LIV6 & 0.55 & 17.11 & 269 & 9.02 & 286 \\
\hline PP-3 & 8/28/01 & RAINBOW & $\mathrm{F}$ & 247 & 140 & LIVER & MT-PP3-LIV7 & 0.64 & 16.82 & 885 & $<1.95$ & 229 \\
\hline PP-3 & $8 / 28 / 01$ & RAINBOW & $\mathrm{M}$ & 268 & 194 & LIVER & MT-PP3-LIV8 & 1.94 & 7.50 & 165 & $<2.2$ & 139 \\
\hline PP-3 & $8 / 28 / 01$ & RAINBOW & M & 265 & 176 & LIVER & MT-PP3-LIV9 & 0.50 & 22.6 & 670 & $<1.97$ & 173 \\
\hline PP-3 & $8 / 28 / 01$ & RAINBOW & $\mathrm{F}$ & 302 & 222 & LIVER & MT-PP3-LIV10 & 0.37 & 49.4 & 608 & 2.54 & 307 \\
\hline PP-3 & $8 / 28 / 01$ & RAINBOW & $\mathrm{F}$ & 302 & 258 & LIVER & MT-PP3-LIV11 & 1.19 & 8.86 & 942 & 2.44 & 180 \\
\hline PP-3 & 8/28/01 & BROOK & $\mathrm{F}$ & 164 & 43 & GILL & & & & & & \\
\hline PP-3 & 8/28/01 & BROOK & M & 167 & 39 & GILL & MT-PP3-GIL1 & 0.47 & 19.4 & 3.97 & 4.02 & 229 \\
\hline PP-3 & $8 / 28 / 01$ & BROOK & M & 134 & 20 & GILL & & & & & & \\
\hline PP-3 & $8 / 28 / 01$ & BROOK & M & 167 & 43 & GILL & & & & & & \\
\hline PP-3 & $8 / 28 / 01$ & BROOK & M & 151 & 32 & GILL & MT-PP3-GIL2 & 0.48 & 19.3 & 3.77 & 6.23 & 314 \\
\hline PP-3 & $8 / 28 / 01$ & BROOK & $\mathrm{F}$ & 139 & 28 & GILL & & & & & & \\
\hline PP-3 & $8 / 28 / 01$ & BROOK & $\mathrm{F}$ & 140 & 22 & GILL & & & & & & \\
\hline PP-3 & $8 / 28 / 01$ & BROOK & M & 163 & 39 & GILL & MT-PP3-GIL3 & 0.35 & 21.1 & 4.14 & 5.01 & 295 \\
\hline PP-3 & $8 / 28 / 01$ & BROOK & M & 169 & 46 & GILL & & & & & & \\
\hline PP-3 & $8 / 28 / 01$ & BROOK & M & 190 & 76 & GILL & MT-PP3-GIL4 & 0.34 & 13.9 & 3.93 & 8.59 & 316 \\
\hline PP-3 & $8 / 28 / 01$ & BROOK & M & 150 & 32 & GILL & & & & & & \\
\hline PP-3 & $8 / 28 / 01$ & BROOK & $\mathrm{F}$ & 153 & 43 & GILL & & & & & & \\
\hline PP-3 & 8/28/01 & BROOK & $\mathrm{F}$ & 147 & 28 & GILL & MT-PP3-GIL5 & 0.20 & 19.9 & 5.79 & 2.74 & 227 \\
\hline PP-3 & $8 / 28 / 01$ & BROOK & M & 151 & 29 & GILL & & & & & & \\
\hline PP-3 & $8 / 28 / 01$ & BROOK & $\mathrm{F}$ & 243 & 140 & GILL & MT-PP3-GIL6 & 0.67 & 5.71 & 5.14 & 17.5 & 257 \\
\hline PP-3 & $8 / 28 / 01$ & RAINBOW & $\mathrm{F}$ & 247 & 140 & GILL & MT-PP3-GIL7 & 0.18 & 16.5 & 3.10 & 3.48 & 8,580 \\
\hline PP-3 & $8 / 28 / 01$ & RAINBOW & $\mathrm{M}$ & 268 & 194 & GILL & MT-PP3-GIL8 & 0.15 & 7.95 & 2.72 & 6.82 & 2,290 \\
\hline PP-3 & $8 / 28 / 01$ & RAINBOW & M & 265 & 176 & GILL & MT-PP3-GIL9 & 0.21 & 26.7 & 3.21 & 6.65 & 2,720 \\
\hline PP-3 & $8 / 28 / 01$ & RAINBOW & $\mathrm{F}$ & 302 & 222 & GILL & MT-PP3-GIL10 & 0.14 & 11.9 & 2.94 & 11.6 & 6,800 \\
\hline PP-3 & $8 / 28 / 01$ & RAINBOW & $\mathrm{F}$ & 302 & 258 & GILL & MT-PP3-GIL11 & 0.31 & 8.54 & 3.88 & 5.79 & 1,580 \\
\hline PP-3 & $8 / 28 / 01$ & BROOK & $\mathrm{F}$ & 164 & 43 & CAECA & & & & & & \\
\hline
\end{tabular}


Individual fish tissues (Continued)

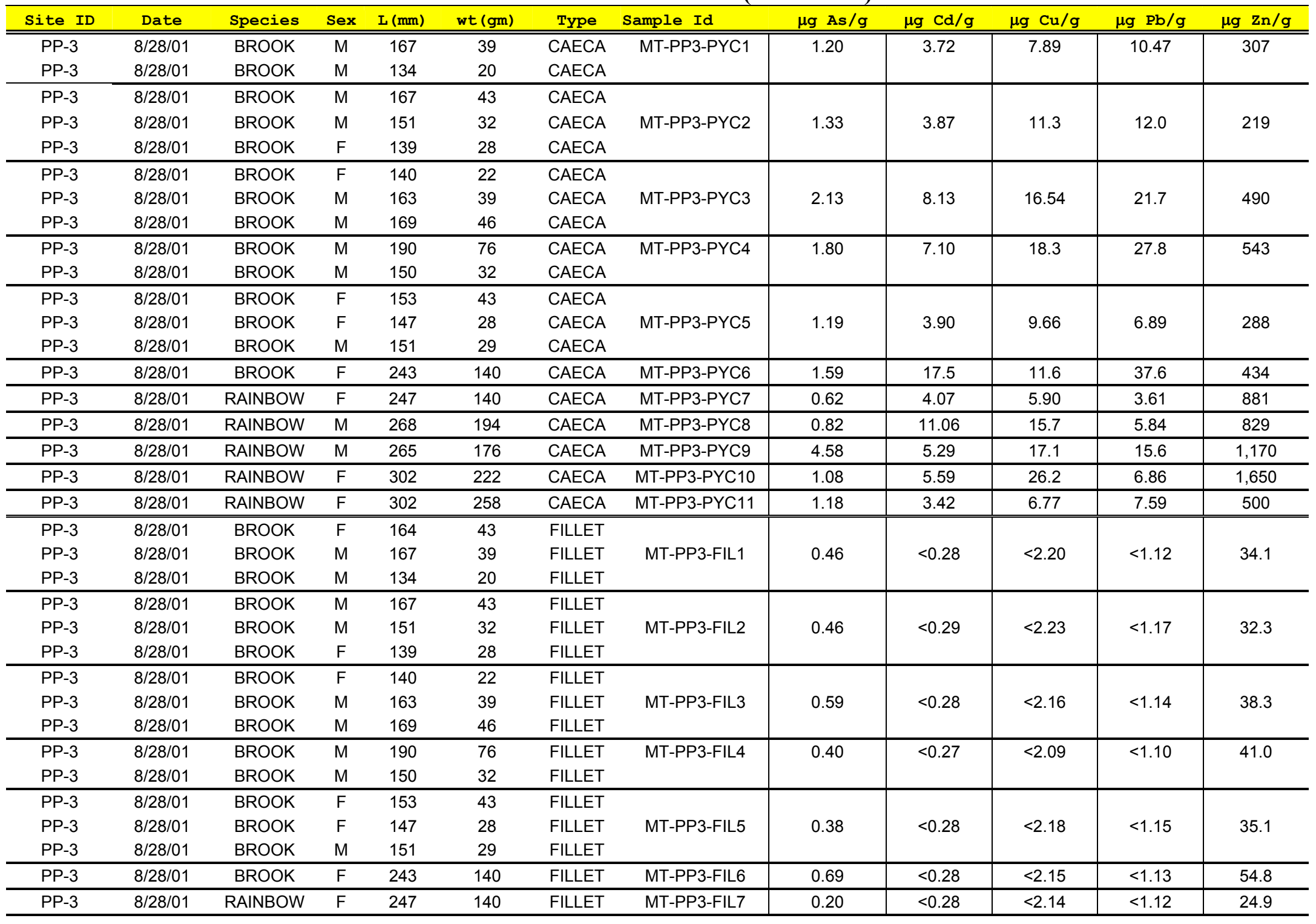


Individual fish tissues (Continued)

\begin{tabular}{|c|c|c|c|c|c|c|c|c|c|c|c|c|}
\hline Site ID & Date & Species & Sex & $\mathrm{L}(\mathrm{mm})$ & wt (gm) & Type & Sample Id & $\mu \mathrm{g}$ As $/ \mathrm{g}$ & $\mu \mathrm{g} \mathrm{Cd} / \mathrm{g}$ & $\mu \mathrm{g} \mathrm{Cu} / \mathrm{g}$ & $\mu \mathrm{g} \mathrm{Pb} / \mathrm{g}$ & $\mu \mathrm{g} \mathrm{zn} / \mathrm{g}$ \\
\hline PP-3 & $8 / 28 / 01$ & RAINBOW & $\bar{M}$ & 268 & 194 & 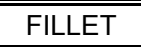 & MT-PP3-FIL8 & 0.25 & $<0.28$ & $<2.15$ & $<1.13$ & 29.0 \\
\hline PP-3 & $8 / 28 / 01$ & RAINBOW & $\mathrm{M}$ & 265 & 176 & FILLET & MT-PP3-FIL9 & 0.29 & $<0.29$ & $<2.10$ & $<1.11$ & 32.9 \\
\hline PP-3 & $8 / 28 / 01$ & RAINBOW & $F$ & 302 & 222 & FILLET & MT-PP3-FIL10 & 0.12 & $<0.28$ & $<2.18$ & $<1.15$ & 32.1 \\
\hline PP-3 & $8 / 28 / 01$ & BROOK & - & 92 & 8 & "WL. FISH & "MT-PP3-WF1 & 1.01 & 0.57 & 5.30 & 5.90 & 370 \\
\hline PP-3 & $8 / 28 / 01$ & BROOK & - & 92 & 7 & WL. FISH & MT-PP3-WF2 & 0.39 & 0.48 & 4.24 & 1.18 & 271 \\
\hline PP-3 & $8 / 28 / 01$ & BROOK & - & 90 & 6 & WL. FISH & MT-PP3-WF3 & 0.77 & 0.89 & 8.32 & 3.59 & 446 \\
\hline PP-3 & $8 / 28 / 01$ & BROOK & - & 94 & 8 & WL. FISH & MT-PP3-WF5 & 0.66 & 0.49 & 5.75 & 1.67 & 239 \\
\hline PP-3 & $8 / 28 / 01$ & RAINBOW & - & 139 & 24 & WL. FISH & MT-PP3-WF6 & 0.35 & 0.31 & 4.61 & 2.23 & 427 \\
\hline PP-3 & $8 / 28 / 01$ & RAINBOW & - & 125 & 17 & WL. FISH & MT-PP3-WF7 & 1.49 & 0.87 & 6.18 & 10.9 & 435 \\
\hline PP-3 & $8 / 28 / 01$ & RAINBOW & - & 165 & 41 & WL. FISH & MT-PP3-WF8 & 0.68 & 0.68 & 6.91 & 3.68 & 308 \\
\hline PP-3 & $8 / 28 / 01$ & RAINBOW & - & 133 & 22 & WL. FISH & MT-PP3-WF9 & 0.64 & 0.52 & 4.37 & 2.01 & 275 \\
\hline PP-3 & $8 / 28 / 01$ & RAINBOW & - & 213 & 96 & WL. FISH & MT-PP3-WF10 & 2.70 & 0.85 & 9.32 & 17.0 & 318 \\
\hline PP-5 & $8 / 28 / 01$ & RAINBOW & $\bar{M}$ & 153 & 37 & LIVER & & & & & & \\
\hline PP-5 & $8 / 28 / 01$ & RAINBOW & M & 167 & 42 & LIVER & MT-PP5-LIV1 & 2.51 & 2.40 & 291 & 13.9 & 190 \\
\hline PP-5 & $8 / 28 / 01$ & RAINBOW & $\bar{F}$ & 155 & 34 & LIVER & MT-PP5-LIV2 & 0.95 & 4.20 & 548 & $<1.90$ & 205 \\
\hline PP-5 & $8 / 28 / 01$ & RAINBOW & $M$ & 220 & 106 & LIVER & & & & & & \\
\hline PP-5 & $8 / 28 / 01$ & RAINBOW & $\bar{M}$ & 222 & 99 & LIVER & MT-PP5-LIV3 & 2.44 & 2.47 & 206 & 3.37 & 162 \\
\hline PP-5 & $8 / 28 / 01$ & RAINBOW & $\mathrm{F}$ & 161 & 43 & LIVER & & & & & & \\
\hline PP-5 & $8 / 28 / 01$ & RAINBOW & $\mathrm{M}$ & 195 & 76 & LIVER & MT-PP5-LIV4 & 2.22 & 3.01 & 454 & 3.89 & 198 \\
\hline PP-5 & $8 / 28 / 01$ & RAINBOW & $\mathrm{F}$ & 167 & 46 & LIVER & & & & & & \\
\hline PP-5 & $8 / 28 / 01$ & RAINBOW & $M$ & 185 & 64 & LIVER & MT-PP5-LIV5 & 2.89 & 3.08 & 176 & $<2.84$ & 216 \\
\hline PP-5 & $8 / 28 / 01$ & RAINBOW & $\mathrm{F}$ & 174 & 49 & LIVER & & & & & & \\
\hline PP-5 & $8 / 28 / 01$ & RAINBOW & $M$ & 173 & 50 & LIVER & MT-PP5-LIV6 & 1.87 & 2.83 & 211 & 4.76 & 159 \\
\hline PP-5 & $8 / 28 / 01$ & RAINBOW & M & 154 & 60 & LIVER & & & & & & \\
\hline PP-5 & $8 / 28 / 01$ & RAINBOW & $\mathrm{M}$ & 182 & 60 & LIVER & MT-PP5-LIV7 & 3.77 & 2.811 & 466 & $<2.79$ & 165 \\
\hline PP-5 & $8 / 28 / 01$ & RAINBOW & $\mathrm{F}$ & 168 & 46 & LIVER & & & & & & \\
\hline PP-5 & $8 / 28 / 01$ & "RAINBOW & $\bar{M}$ & 153 & 37 & 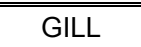 & & & & & & \\
\hline PP-5 & $8 / 28 / 01$ & RAINBOW & M & 167 & 42 & GILL & MT-PP5-GIL1 & 0.50 & 5.69 & 3.77 & 3.86 & 665 \\
\hline PP-5 & $8 / 28 / 01$ & RAINBOW & $\mathrm{M}$ & 154 & 36 & GILL & & & & & & \\
\hline PP-5 & $8 / 28 / 01$ & RAINBOW & $\bar{F}$ & 155 & 34 & GILL & MT-PP5-GIL2 & 0.41 & 3.74 & 4.85 & 3.68 & 1380 \\
\hline
\end{tabular}


Individual fish tissues (Continued)

\begin{tabular}{|c|c|c|c|c|c|c|c|c|c|c|c|c|}
\hline Site ID & Date & Species & Sex & $\mathrm{L}(\mathrm{mm})$ & wt (gm) & Type & Sample Id & $\mu \mathrm{g}$ As $/ \mathrm{g}$ & $\mu \mathrm{g} \mathrm{Cd} / \mathrm{g}$ & $\mu \mathrm{g} \mathrm{Cu} / \mathrm{g}$ & $\mu \mathrm{g} \mathrm{Pb} / \mathrm{g}$ & $\mu \mathrm{g} \mathrm{Zn} / \mathrm{g}$ \\
\hline PP-5 & $8 / 28 / 01$ & RAINBOW & $\bar{F}$ & 161 & 43 & 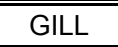 & & & & & & \\
\hline PP-5 & $8 / 28 / 01$ & RAINBOW & $M$ & 195 & 76 & GILL & MT-PP5-GIL4 & 0.59 & 5.36 & 3.28 & $<2.68$ & 1184 \\
\hline PP-5 & $8 / 28 / 01$ & RAINBOW & $\mathrm{F}$ & 167 & 46 & GILL & & & & & & \\
\hline PP-5 & $8 / 28 / 01$ & RAINBOW & $M$ & 185 & 64 & GILL & MT-PP5-GIL5 & 0.39 & 4.73 & 5.22 & 5.00 & 837 \\
\hline PP-5 & $8 / 28 / 01$ & RAINBOW & $\mathrm{F}$ & 174 & 49 & GILL & & & & & & \\
\hline PP-5 & $8 / 28 / 01$ & RAINBOW & $M$ & 173 & 50 & GILL & MT-PP5-GIL6 & 0.47 & 7.25 & 3.08 & 4.23 & 829 \\
\hline PP-5 & 8/28/01 & RAINBOW & M & 154 & 60 & GILL & & & & & & \\
\hline PP-5 & 8/28/01 & RAINBOW & M & 182 & 60 & GILL & MT-PP5-LIV7 & 0.55 & 8.44 & 3.25 & 6.18 & 237 \\
\hline PP-5 & 8/28/01 & RAINBOW & $\mathrm{F}$ & 168 & 46 & GILL & & & & & & \\
\hline PP-5 & $8 / 28 / 01$ & RAINBOW & $M$ & 153 & 37 & CAECA & & & & & & \\
\hline PP-5 & $8 / 28 / 01$ & RAINBOW & M & 167 & 42 & CAECA & MT-PP5-PYC1 & 3.19 & 2.29 & 11.5 & 8.64 & 536 \\
\hline PP-5 & 8/28/01 & RAINBOW & M & 154 & 36 & CAECA & & & & & & \\
\hline PP-5 & $8 / 28 / 01$ & RAINBOW & $F$ & 155 & 34 & CAECA & MT-PP5-PYC2 & 2.48 & 2.13 & 8.73 & 4.64 & 571 \\
\hline PP-5 & $8 / 28 / 01$ & RAINBOW & M & 220 & 106 & CAECA & & & & & & \\
\hline PP-5 & $8 / 28 / 01$ & RAINBOW & $M$ & 222 & 99 & CAECA & MT-PP5-PYC3 & 3.29 & 2.00 & 9.37 & 12.6 & 827 \\
\hline PP-5 & $8 / 28 / 01$ & RAINBOW & $\mathrm{F}$ & 161 & 43 & CAECA & & & & & & \\
\hline PP-5 & $8 / 28 / 01$ & RAINBOW & $M$ & 195 & 76 & CAECA & MT-PP5-PYC4 & 3.95 & 2.11 & 9.44 & 12.4 & 734 \\
\hline PP-5 & $8 / 28 / 01$ & RAINBOW & $\mathrm{F}$ & 167 & 46 & CAECA & & & & & & \\
\hline PP-5 & $8 / 28 / 01$ & RAINBOW & $M$ & 185 & 64 & CAECA & MT-PP5-PYC5 & 4.98 & 2.68 & 9.67 & 8.45 & 856 \\
\hline PP-5 & $8 / 28 / 01$ & RAINBOW & $\mathrm{F}$ & 174 & 49 & CAECA & & & & & & \\
\hline PP-5 & $8 / 28 / 01$ & RAINBOW & $M$ & 173 & 50 & CAECA & MT-PP5-PYC6 & 3.49 & 3.94 & 10.42 & 4.80 & 580 \\
\hline PP-5 & 8/28/01 & RAINBOW & $\mathrm{M}$ & 154 & 60 & CAECA & & & & & & \\
\hline PP-5 & $8 / 28 / 01$ & RAINBOW & $M$ & 182 & 60 & CAECA & MT-PP5-PYC7 & 3.97 & 2.99 & 17.29 & 7.31 & 943 \\
\hline PP-5 & $8 / 28 / 01$ & RAINBOW & $\mathrm{F}$ & 168 & 46 & CAECA & & & & & & \\
\hline PP-5 & $8 / 28 / 01$ & RAINBOW & $M$ & 153 & 37 & FILLET & & & & & & \\
\hline PP-5 & $8 / 28 / 01$ & RAINBOW & M & 167 & 42 & FILLET & MT-PP5-FIL1 & 0.36 & $<0.28$ & $<2.15$ & 2.30 & 38.1 \\
\hline PP-5 & $8 / 28 / 01$ & RAINBOW & M & 154 & 36 & FILLET & & & & & & \\
\hline PP-5 & $8 / 28 / 01$ & RAINBOW & $F$ & 155 & 34 & FILLET & MT-PP5-FIL2 & 0.38 & $<0.28$ & $<2.11$ & $<1.11$ & 36.6 \\
\hline PP-5 & $8 / 28 / 01$ & RAINBOW & M & 220 & 106 & FILLET & & & & & & \\
\hline PP-5 & $8 / 28 / 01$ & RAINBOW & $M$ & 222 & 99 & FILLET & MT-PP5-FIL3 & 0.41 & $<0.28$ & $<2.18$ & $<1.15$ & 35.4 \\
\hline PP-5 & $8 / 28 / 01$ & RAINBOW & $\mathrm{F}$ & 161 & 43 & FILLET & & & & & & \\
\hline PP-5 & $8 / 28 / 01$ & RAINBOW & $M$ & 195 & 76 & FILLET & MT-PP5-FIL4 & 0.33 & 1.62 & $<2.20$ & $<1.16$ & 36.8 \\
\hline PP-5 & $8 / 28 / 01$ & RAINBOW & $\mathrm{F}$ & 167 & 46 & FILLET & & & & & & \\
\hline PP-5 & $8 / 28 / 01$ & RAINBOW & $M$ & 185 & 64 & FILLET & MT-PP5-FIL5 & 0.47 & $<0.28$ & $<2.14$ & $<1.12$ & 44.0 \\
\hline PP-5 & $8 / 28 / 01$ & RAINBOW & $\mathrm{F}$ & 174 & 49 & FILLET & & & & & & \\
\hline PP-5 & $8 / 28 / 01$ & RAINBOW & $M$ & 173 & 50 & FILLET & MT-PP5-FIL6 & 1.51 & 0.61 & 4.21 & 3.11 & 294 \\
\hline
\end{tabular}


Individual fish tissues (Continued)

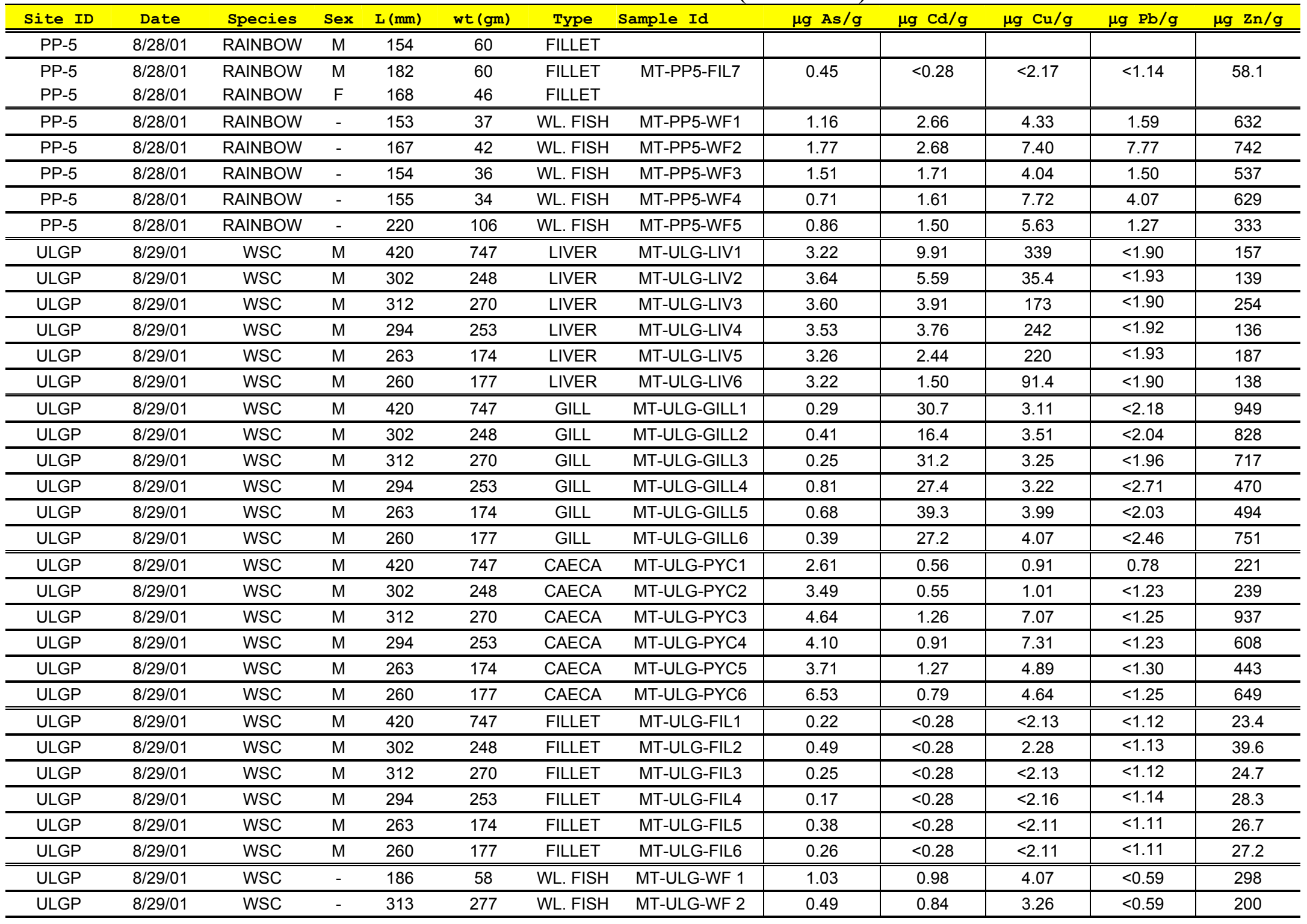


Individual fish tissues (Continued)

\begin{tabular}{|c|c|c|c|c|c|c|c|c|c|c|c|c|}
\hline Site ID & Date & Species & Sex & $\mathrm{L}(\mathrm{mm})$ & wt (gm) & Type & Sample Id & $\mu \mathrm{g}$ As/g & $\mu \mathrm{g} \mathrm{Cd} / \mathrm{g}$ & $\mu \mathrm{g} \mathrm{Cu} / \mathrm{g}$ & $\mu \mathrm{g} \mathrm{Pb} / \mathrm{g}$ & $\mu \mathrm{g} \mathrm{Zn} / \mathrm{g}$ \\
\hline ULGP & $8 / 29 / 01$ & WSC & - & 370 & 422 & WL. FISH & MT-ULG-WF 3 & 0.22 & 1.03 & 10.7 & $<0.59$ & 248 \\
\hline WS-3 & $8 / 27 / 01$ & BROOK & $M$ & 127 & 23 & LIVER & \multirow{6}{*}{ MT-WS3-LIV1 } & \multirow{6}{*}{2.31} & \multirow{6}{*}{13.58} & \multirow{6}{*}{169} & \multirow{6}{*}{$<2.87$} & \multirow{6}{*}{246} \\
\hline WS-3 & $8 / 27 / 01$ & BROOK & - & 93 & 10 & LIVER & & & & & & \\
\hline WS-3 & $8 / 27 / 01$ & BROOK & $\mathrm{F}$ & 111 & 12 & LIVER & & & & & & \\
\hline WS-3 & $8 / 27 / 01$ & BROOK & $\mathrm{F}$ & 114 & 15 & LIVER & & & & & & \\
\hline WS-3 & $8 / 27 / 01$ & BROOK & - & 100 & 9 & LIVER & & & & & & \\
\hline WS-3 & $8 / 27 / 01$ & BROOK & $\mathrm{F}$ & 114 & 17 & LIVER & & & & & & \\
\hline WS-3 & $8 / 27 / 01$ & BROOK & $F$ & 142 & 28 & LIVER & \multirow{6}{*}{ MT-WS3-LIV2 } & \multirow{6}{*}{2.67} & \multirow{6}{*}{8.46} & \multirow{6}{*}{137} & \multirow{6}{*}{$<2.25$} & \multirow{6}{*}{231} \\
\hline WS-3 & $8 / 27 / 01$ & BROOK & M & 115 & 14 & LIVER & & & & & & \\
\hline WS-3 & $8 / 27 / 01$ & BROOK & - & 97 & 8 & LIVER & & & & & & \\
\hline WS-3 & $8 / 27 / 01$ & BROOK & $M$ & 110 & 14 & LIVER & & & & & & \\
\hline WS-3 & $8 / 27 / 01$ & BROOK & M & 112 & 13 & LIVER & & & & & & \\
\hline WS-3 & $8 / 27 / 01$ & BROOK & - & 105 & 9 & LIVER & & & & & & \\
\hline WS-3 & $8 / 27 / 01$ & BROOK & $M$ & 120 & 19 & LIVER & \multirow{6}{*}{ MT-WS3-LIV3 } & \multirow{6}{*}{5.14} & \multirow{6}{*}{9.58} & \multirow{6}{*}{149} & \multirow{6}{*}{5.10} & \multirow{6}{*}{218} \\
\hline WS-3 & $8 / 27 / 01$ & BROOK & M & 132 & 26 & LIVER & & & & & & \\
\hline WS-3 & $8 / 27 / 01$ & BROOK & $\mathrm{F}$ & 100 & 9 & LIVER & & & & & & \\
\hline WS-3 & $8 / 27 / 01$ & BROOK & - & 105 & 9 & LIVER & & & & & & \\
\hline WS-3 & $8 / 27 / 01$ & BROOK & M & 102 & 10 & LIVER & & & & & & \\
\hline WS-3 & $8 / 27 / 01$ & BROOK & - & 108 & 12 & LIVER & & & & & & \\
\hline WS-3 & $8 / 27 / 01$ & BROOK & - & 111 & 13 & LIVER & \multirow{6}{*}{ MT-WS3-LIV4 } & \multirow{6}{*}{3.62} & \multirow{6}{*}{9.79} & \multirow{6}{*}{190} & \multirow{6}{*}{5.66} & \\
\hline WS-3 & $8 / 27 / 01$ & BROOK & M & 125 & 20 & LIVER & & & & & & \\
\hline WS-3 & $8 / 27 / 01$ & BROOK & M & 112 & 12 & LIVER & & & & & & 274 \\
\hline WS-3 & $8 / 27 / 01$ & BROOK & - & 109 & 10 & LIVER & & & & & & \\
\hline WS-3 & $8 / 27 / 01$ & BROOK & M & 110 & 13 & LIVER & & & & & & \\
\hline WS-3 & $8 / 27 / 01$ & BROOK & - & 98 & 9 & LIVER & & & & & & \\
\hline WS-3 & $8 / 27 / 01$ & BROOK & - & 105 & 9 & LIVER & & & & & & \\
\hline WS-3 & $8 / 27 / 01$ & BROOK & M & 127 & 23 & GILL & & & & & & \\
\hline WS-3 & 8/27/01 & BROOK & - & 93 & 10 & GILL & & & & & & \\
\hline WS-3 & $8 / 27 / 01$ & BROOK & $\mathrm{F}$ & 111 & 12 & GILL & MT-WS3-GIL1 & 2.31 & 29.8 & 4.36 & $<3.92$ & 382 \\
\hline WS-3 & $8 / 27 / 01$ & BROOK & $\mathrm{F}$ & 114 & 15 & GILL & & & & & & \\
\hline WS-3 & $8 / 27 / 01$ & BROOK & - & 100 & 9 & GILL & & & & & & \\
\hline WS-3 & 8/27/01 & BROOK & $\mathrm{F}$ & 114 & 17 & GILL & & & & & & \\
\hline WS-3 & $8 / 27 / 01$ & BROOK & $\mathrm{F}$ & 142 & 28 & $\overline{\text { GILL }}$ & & & & & & \\
\hline WS-3 & $8 / 27 / 01$ & BROOK & M & 115 & 14 & GILL & & & & & & \\
\hline WS-3 & 8/27/01 & BROOK & - & 97 & 8 & GILL & MT-WS3-GIL2 & 6.70 & 28.6 & 4.37 & $<2.82$ & 344 \\
\hline WS-3 & $8 / 27 / 01$ & BROOK & M & 110 & 14 & GILL & & & & & & \\
\hline WS-3 & $8 / 27 / 01$ & BROOK & M & 112 & 13 & GILL & & & & & & \\
\hline WS-3 & $8 / 27 / 01$ & BROOK & - & 105 & 9 & GILL & & & & & & \\
\hline
\end{tabular}


Individual fish tissues (Continued)

\begin{tabular}{|c|c|c|c|c|c|c|c|c|c|c|c|c|}
\hline Site ID & Date & Species & Sex & $\mathrm{L}(\mathrm{mm})$ & wt (gm) & Type & Sample Id & $\mu \mathrm{g}$ As/g & $\mu \mathrm{g} \mathrm{Cd} / \mathrm{g}$ & $\mu \mathrm{g} \mathrm{Cu} / \mathrm{g}$ & $\mu \mathrm{g} \mathrm{Pb} / \mathrm{g}$ & $\mu \mathrm{g} \mathrm{Zn} / \mathrm{g}$ \\
\hline WS-3 & $8 / 27 / 01$ & BROOK & $\bar{M}$ & 120 & 19 & 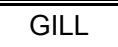 & \multirow{6}{*}{ MT-WS3-GIL3 } & \multirow{6}{*}{4.06} & \multirow{6}{*}{27.0} & \multirow{6}{*}{4.77} & \multirow{6}{*}{$<2.84$} & \multirow{6}{*}{290.2} \\
\hline WS-3 & $8 / 27 / 01$ & BROOK & $M$ & 132 & 26 & GILL & & & & & & \\
\hline WS-3 & 8/27/01 & BROOK & $\mathrm{F}$ & 100 & 9 & GILL & & & & & & \\
\hline WS-3 & $8 / 27 / 01$ & BROOK & - & 105 & 9 & GILL & & & & & & \\
\hline WS-3 & $8 / 27 / 01$ & BROOK & M & 102 & 10 & GILL & & & & & & \\
\hline WS-3 & $8 / 27 / 01$ & BROOK & - & 108 & 12 & GILL & & & & & & \\
\hline WS-3 & $8 / 27 / 01$ & BROOK & - & 111 & 13 & GILL & \multirow{7}{*}{ MT-WS3-GIL4 } & \multirow{7}{*}{3.61} & \multirow{7}{*}{24.2} & \multirow{7}{*}{4.35} & \multirow{7}{*}{$<2.71$} & \multirow{7}{*}{297} \\
\hline WS-3 & 8/27/01 & BROOK & M & 125 & 20 & GILL & & & & & & \\
\hline WS-3 & $8 / 27 / 01$ & BROOK & M & 112 & 12 & GILL & & & & & & \\
\hline WS-3 & $8 / 27 / 01$ & BROOK & - & 109 & 10 & GILL & & & & & & \\
\hline WS-3 & $8 / 27 / 01$ & BROOK & M & 110 & 13 & GILL & & & & & & \\
\hline WS-3 & $8 / 27 / 01$ & BROOK & - & 105 & 9 & GILL & & & & & & \\
\hline WS-3 & $8 / 27 / 01$ & BROOK & - & 98 & 9 & GILL & & & & & & \\
\hline WS-3 & $8 / 27 / 01$ & "BROOK & $\bar{M}$ & 127 & 23 & CAECA & \multirow{6}{*}{ MT-WS3-PYC1 } & \multirow{6}{*}{31.1} & \multirow{6}{*}{6.20} & \multirow{6}{*}{8.57} & \multirow{6}{*}{3.97} & \multirow{6}{*}{337} \\
\hline WS-3 & 8/27/01 & BROOK & - & 93 & 10 & CAECA & & & & & & \\
\hline WS-3 & $8 / 27 / 01$ & BROOK & $\mathrm{F}$ & 111 & 12 & CAECA & & & & & & \\
\hline WS-3 & $8 / 27 / 01$ & BROOK & $\mathrm{F}$ & 114 & 15 & CAECA & & & & & & \\
\hline WS-3 & $8 / 27 / 01$ & BROOK & - & 100 & 9 & CAECA & & & & & & \\
\hline WS-3 & $8 / 27 / 01$ & BROOK & $\mathrm{F}$ & 114 & 17 & CAECA & & & & & & \\
\hline WS-3 & $8 / 27 / 01$ & BROOK & $\mathrm{F}$ & 142 & 28 & CAECA & \multirow{6}{*}{ MT-WS3-PYC2 } & \multirow{6}{*}{20.9} & \multirow{6}{*}{7.04} & \multirow{6}{*}{9.84} & \multirow{6}{*}{$<3.39$} & \\
\hline WS-3 & $8 / 27 / 01$ & BROOK & M & 115 & 14 & CAECA & & & & & & \\
\hline WS-3 & $8 / 27 / 01$ & BROOK & - & 97 & 8 & CAECA & & & & & & 369 \\
\hline WS-3 & $8 / 27 / 01$ & BROOK & M & 110 & 14 & CAECA & & & & & & \\
\hline WS-3 & $8 / 27 / 01$ & BROOK & M & 112 & 13 & CAECA & & & & & & \\
\hline WS-3 & $8 / 27 / 01$ & BROOK & - & 105 & 9 & CAECA & & & & & & \\
\hline WS-3 & $8 / 27 / 01$ & BROOK & $M$ & 120 & 19 & CAECA & & & & & & \\
\hline WS-3 & $8 / 27 / 01$ & BROOK & M & 132 & 26 & CAECA & & & & & & \\
\hline WS-3 & $8 / 27 / 01$ & BROOK & $\mathrm{F}$ & 100 & 9 & CAECA & MT-WS3-PYC3 & 22.6 & 4.70 & 7.52 & $<3.54$ & 331 \\
\hline WS-3 & $8 / 27 / 01$ & BROOK & - & 105 & 9 & CAECA & & & & & & \\
\hline WS-3 & $8 / 27 / 01$ & BROOK & M & 102 & 10 & CAECA & & & & & & \\
\hline WS-3 & $8 / 27 / 01$ & BROOK & - & 108 & 12 & CAECA & & & & & & \\
\hline WS-3 & $8 / 27 / 01$ & BROOK & - & 111 & 13 & CAECA & & & & & & \\
\hline WS-3 & $8 / 27 / 01$ & BROOK & M & 125 & 20 & CAECA & & & & & & \\
\hline WS-3 & $8 / 27 / 01$ & BROOK & M & 112 & 12 & CAECA & MT-WS3-PYC4 & 27.3 & 6.54 & 6.12 & $<4.20$ & 395 \\
\hline WS-3 & $8 / 27 / 01$ & BROOK & - & 109 & 10 & CAECA & & & & & & \\
\hline WS-3 & $8 / 27 / 01$ & BROOK & M & 110 & 13 & CAECA & & & & & & \\
\hline WS-3 & $8 / 27 / 01$ & BROOK & - & 105 & 9 & CAECA & & & & & & \\
\hline WS-3 & $8 / 27 / 01$ & BROOK & - & 98 & 97 & CAECA & & & & & & \\
\hline WS-3 & $8 / 8 / 27 / 01$ & BROOK & $\bar{M}$ & 127 & 23 & CAECA & & & & & & \\
\hline
\end{tabular}


Individual fish tissues (Continued)

\begin{tabular}{|c|c|c|c|c|c|c|c|c|c|c|c|c|}
\hline Site ID & Date & Species & Sex & $\mathrm{L}(\mathrm{mm})$ & wt (gm) & Type & Sample Id & $\mu \mathrm{g}$ As/g & $\mu \mathrm{g} \mathrm{Cd} / \mathrm{g}$ & $\mu \mathrm{g} \mathrm{Cu} / \mathrm{g}$ & $\mu \mathrm{g} \mathrm{Pb} / \mathrm{g}$ & $\mu \mathrm{g} \mathrm{Zn} / \mathrm{g}$ \\
\hline WS-3 & $8 / 27 / 01$ & BROOK & - & 93 & 10 & $\begin{array}{l}\text { FILET } \\
\end{array}$ & \multirow{5}{*}{ MT-WS3-FIL1 } & \multirow{5}{*}{2.02} & \multirow{5}{*}{$<0.28$} & \multirow{5}{*}{2.13} & \multirow{5}{*}{$<1.12$} & \multirow{5}{*}{47.4} \\
\hline WS-3 & $8 / 27 / 01$ & BROOK & $\mathrm{F}$ & 111 & 12 & FILET & & & & & & \\
\hline WS-3 & 8/27/01 & BROOK & $\mathrm{F}$ & 114 & 15 & FILET & & & & & & \\
\hline WS-3 & 8/27/01 & BROOK & - & 100 & 9 & FILET & & & & & & \\
\hline WS-3 & 8/27/01 & BROOK & $\mathrm{F}$ & 114 & 17 & FILET & & & & & & \\
\hline WS-3 & $8 / 27 / 01$ & BROOK & $\bar{F}$ & 142 & 28 & FILET & \multirow{6}{*}{ MT-WS3-FIL2 } & \multirow{6}{*}{1.87} & \multirow{6}{*}{$<0.28$} & \multirow{6}{*}{$<2.13$} & \multirow{6}{*}{$<1.12$} & \multirow{6}{*}{41.8} \\
\hline WS-3 & 8/27/01 & BROOK & M & 115 & 14 & FILET & & & & & & \\
\hline WS-3 & $8 / 27 / 01$ & BROOK & - & 97 & 8 & FILET & & & & & & \\
\hline WS-3 & 8/27/01 & BROOK & $M$ & 110 & 14 & FILET & & & & & & \\
\hline WS-3 & $8 / 27 / 01$ & BROOK & M & 112 & 13 & FILET & & & & & & \\
\hline WS-3 & $8 / 27 / 01$ & BROOK & - & 105 & 9 & FILET & & & & & & \\
\hline WS-3 & $8 / 27 / 01$ & BROOK & $M$ & 120 & 19 & FILET & \multirow{6}{*}{ MT-WS3-FIL3 } & \multirow{6}{*}{1.69} & \multirow{6}{*}{$<0.28$} & \multirow{6}{*}{$<2.16$} & \multirow{6}{*}{$<1.14$} & \multirow{6}{*}{47.8} \\
\hline WS-3 & $8 / 27 / 01$ & BROOK & M & 132 & 26 & FILET & & & & & & \\
\hline WS-3 & $8 / 27 / 01$ & BROOK & $\mathrm{F}$ & 100 & 9 & FILET & & & & & & \\
\hline WS-3 & $8 / 27 / 01$ & BROOK & - & 105 & 9 & FILET & & & & & & \\
\hline WS-3 & $8 / 27 / 01$ & BROOK & $M$ & 102 & 10 & FILET & & & & & & \\
\hline WS-3 & $8 / 27 / 01$ & BROOK & - & 108 & 12 & FILET & & & & & & \\
\hline WS-3 & $8 / 27 / 01$ & BROOK & - & 111 & 13 & FILET & \multirow{7}{*}{ MT-WS3-FIL4 } & \multirow{7}{*}{1.97} & \multirow{7}{*}{$<0.28$} & \multirow{7}{*}{$<2.10$} & \multirow{7}{*}{$<1.12$} & \multirow{7}{*}{58.9} \\
\hline WS-3 & $8 / 27 / 01$ & BROOK & M & 125 & 20 & FILET & & & & & & \\
\hline WS-3 & $8 / 27 / 01$ & BROOK & M & 112 & 12 & FILET & & & & & & \\
\hline WS-3 & 8/27/01 & BROOK & - & 109 & 10 & FILET & & & & & & \\
\hline WS-3 & $8 / 27 / 01$ & BROOK & $M$ & 110 & 13 & FILET & & & & & & \\
\hline WS-3 & $8 / 27 / 01$ & BROOK & - & 105 & 9 & FILET & & & & & & \\
\hline WS-3 & $8 / 27 / 01$ & BROOK & - & 98 & 9 & FILET & & & & & & \\
\hline WS-3 & $8 / 27 / 01$ & BROOK & - & 92 & 6 & WL. FISH & MT-WS3-WF1 & 7.94 & 2.66 & 4.33 & 1.59 & 632 \\
\hline WS-3 & $8 / 27 / 01$ & BROOK & - & 94 & 8 & WL. FISH & MT-WS3-WF2 & 17.0 & 2.68 & 7.40 & 7.77 & 742 \\
\hline WS-3 & $8 / 27 / 01$ & BROOK & - & 94 & 7 & WL. FISH & MT-WS3-WF3 & 7.86 & 1.71 & 4.04 & 1.50 & 537 \\
\hline WS-3 & $8 / 27 / 01$ & BROOK & - & 135 & 23 & WL. FISH & MT-WS3-WF4 & 17.2 & 1.61 & 7.72 & 4.07 & 629 \\
\hline WS-3 & $8 / 27 / 01$ & BROOK & - & 146 & 34 & WL. FISH & MT-WS3-WF5 & 3.83 & 1.50 & 5.63 & 1.27 & 333 \\
\hline
\end{tabular}


Histological analysis

\begin{tabular}{|c|c|c|c|c|c|c|c|c|c|c|c|}
\hline $\begin{array}{l}\text { Site ID } \\
\text { (n) }\end{array}$ & Species & Tissue & $\begin{array}{c}\text { Glycogen } \\
\text { Vacuolation }\end{array}$ & $\begin{array}{c}\text { Scattered } \\
\text { Degeneration } \\
\text { Hepatocytes }\end{array}$ & $\begin{array}{c}\text { Focal } \\
\text { Inflammation }\end{array}$ & $\begin{array}{c}\text { Nuclear } \\
\text { Vacuolation }\end{array}$ & $\begin{array}{l}\text { Karryorrhetic } \\
\text { Nuclei }\end{array}$ & Hepatomegally & $\begin{array}{l}\text { Hyper- } \\
\text { cellularity }\end{array}$ & $\begin{array}{l}\text { Melano- } \\
\text { macraphage } \\
\text { Centers }\end{array}$ & Comments \\
\hline $\begin{array}{l}P P-1 \\
(n=15)\end{array}$ & Brook & Gill & & & & & & & & & Mostly normal \\
\hline $\begin{array}{l}P P-1 \\
(n=15)\end{array}$ & Brook & Liver & +2 & $\begin{array}{c}+3 \\
\text { (9 of } 15 \text { fish) } \\
\end{array}$ & $\begin{array}{c}+2 \\
\text { (9 of } 15 \text { fish) } \\
\end{array}$ & $\begin{array}{c}+2 \\
\text { (3 of } 15 \text { fish) } \\
\end{array}$ & & & & & \\
\hline $\begin{array}{l}\mathrm{PP}-1 \\
(\mathrm{n}=15\end{array}$ & Brook & Kidney & & & & & & & & +3 & Regenerating tubules +2 \\
\hline $\begin{array}{l}\mathrm{PP}-1 \\
(\mathrm{n}=15) \\
\end{array}$ & Brook & Spleen & & & & & & & & +2 & \\
\hline $\begin{array}{l}P P-1 \\
(n=15)\end{array}$ & Brook & G.I. & - & & & & & & & & $\begin{array}{l}\text { Contained feed;migrating lymphocytes } \\
\text { epithelia }+2 \text {; rodlet cells }\end{array}$ \\
\hline $\begin{array}{l}\text { PP-1 } \\
(n=15)\end{array}$ & Brook & $\begin{array}{c}\text { Skel. } \\
\text { Muscle }\end{array}$ & - & & & & & & & & Focal areas of fatty infiltration \\
\hline PP-1 & Brook & $\begin{array}{c}\text { General } \\
\text { Condition }\end{array}$ & \multicolumn{9}{|c|}{5 fish scored 0 for glycogen Vacuolation, highly variable, more than any other group. } \\
\hline $\begin{array}{l}P P-3 \\
(n=15)\end{array}$ & Brook & Gill & - & & & & & & & & Mostly normal \\
\hline $\begin{array}{l}\text { PP-3 } \\
(n=15)\end{array}$ & Brook & Liver & 0 & +3 & +2.5 & +2 & (5 fish) & $\begin{array}{c}+3 \\
(2 \text { of } 15 \text { fish })\end{array}$ & +4 & & \\
\hline $\begin{array}{l}P P-3 \\
(n=15)\end{array}$ & Brook & Kidney & - & & & & & & & +3.5 & $\begin{array}{l}\text { Hypertrophy tubule epithelia; } 1 \text { fish } \\
\text { Degenerate tubule epithelia }\end{array}$ \\
\hline $\begin{array}{l}\text { PP-3 } \\
(n=15)\end{array}$ & Brook & Spleen & - & & & & & & & +3 & \\
\hline $\begin{array}{l}P P-3 \\
(n=15)\end{array}$ & Brook & G.I. & - & & & & & & & & $\begin{array}{l}\text { Empty to packed with feed, migrating } \\
\text { lymphocytes mucosal epithelia }+2.5\end{array}$ \\
\hline $\begin{array}{l}\text { PP-3 } \\
(n=15)\end{array}$ & Brook & $\begin{array}{c}\text { Skel. } \\
\text { Muscle }\end{array}$ & - & & & & & & & & $\begin{array}{l}\text { Scattered degeneration and fatty } \\
\text { inflitration }\end{array}$ \\
\hline PP-3 & Brook & $\begin{array}{c}\text { General } \\
\text { Condition } \\
\end{array}$ & \multicolumn{9}{|c|}{$\begin{array}{l}\text { Kidney contains sloughed cells and calcification }+2 \text {, focal inflammation, hypercellular glomeruli. Atrophy (moderately severe hypercellularity) liver and kidney; } \\
\text { starvation and contaminant burden. Chronic cell turnover (melanomacrophage centers) moderate in spleen and somewhat more severe in kidney. }\end{array}$} \\
\hline $\begin{array}{l}\text { PP-3 } \\
(n=5)\end{array}$ & Rainbow & Gill & - & & & & & & & & Mostly normal \\
\hline $\begin{array}{l}\mathrm{PP}-3 \\
(\mathrm{n}=5)\end{array}$ & Rainbow & Liver & 0 & +3 & & & & & +4 & +3 & $\begin{array}{l}\text { Vasculitus }+3 \text {, scat. foci granulomatous infl.; } \\
\text { Increase in bile ducts \& inflammation }\end{array}$ \\
\hline
\end{tabular}


Histological analysis (Continued)

\begin{tabular}{|c|c|c|c|c|c|c|c|c|c|c|c|}
\hline $\begin{array}{l}\text { Site ID } \\
\text { (n) }\end{array}$ & Species & Tissue & $\begin{array}{c}\text { Glycogen } \\
\text { Vacuolation }\end{array}$ & $\begin{array}{c}\text { Scattered } \\
\text { Degeneration } \\
\text { Hepatocytes }\end{array}$ & $\begin{array}{c}\text { Focal } \\
\text { Inflammation }\end{array}$ & $\begin{array}{l}\text { Nuclear } \\
\text { Vacuolation }\end{array}$ & $\begin{array}{l}\text { Karryorrhetic } \\
\text { Nuclei }\end{array}$ & Hepatomegally & $\begin{array}{l}\text { Hyper- } \\
\text { cellularity }\end{array}$ & $\begin{array}{l}\text { Melano- } \\
\text { macraphage } \\
\text { Centers }\end{array}$ & Comments \\
\hline $\begin{array}{l}\text { PP-3 } \\
(n=5)\end{array}$ & Rainbow & Kidney & & & & & & & & +5 & $\begin{array}{l}\text { Erythrophagia }+3 \text {; dystrophic calcification } \\
\text { Tubule lumen }+2 \text { hypercellular glomeruli }\end{array}$ \\
\hline $\begin{array}{l}\mathrm{PP}-3 \\
(\mathrm{n}=5)\end{array}$ & Rainbow & Spleen & & & & & & & & +4 & Few erythrocytes \\
\hline $\begin{array}{l}\mathrm{PP}-3 \\
(\mathrm{n}=5)\end{array}$ & Rainbow & G.I. & & & & & & & & & $\begin{array}{l}\text { Empty to some feed; migrating lymphocytes } \\
\text { mucosal epithelia }+3\end{array}$ \\
\hline $\begin{array}{l}\mathrm{PP}-3 \\
(\mathrm{n}=5)\end{array}$ & Rainbow & $\begin{array}{l}\text { Skel. } \\
\text { Muscle }\end{array}$ & & & & & & & & & Scattered degeneration +2 \\
\hline $\begin{array}{l}\mathrm{PP}-3 \\
(\mathrm{n}=5) \\
\end{array}$ & Rainbow & General & $\begin{array}{r}\text { Increased } \\
\text { accumul }\end{array}$ & $\begin{array}{l}\text { mucous cells } \mathrm{i} \\
\text { ation of melano }\end{array}$ & $\begin{array}{l}\text { in the skin (seve } \\
\text { macrophage ce }\end{array}$ & eral layers thich & $\begin{array}{l}\text { k) The most se } \\
\text { roup with moder } \\
\text { cholangitis (inf }\end{array}$ & $\begin{array}{l}\text { evere changes se } \\
\text { rate to moderatel } \\
\text { flammation of the }\end{array}$ & $\begin{array}{l}\text { en in this } \mathrm{g} \\
\text { ly severe } \mathrm{m} \\
\text { bile ducts) }\end{array}$ & $\begin{array}{l}\text { yroup. Interstiti } \\
\text { nelanomacroph } \\
\text { ). }\end{array}$ & $\begin{array}{l}\text { al tissue in kidney almost solid black from } \\
\text { lage centers in liver and spleen, and with }\end{array}$ \\
\hline $\begin{array}{l}\text { PP-5 } \\
(n=15)\end{array}$ & Rainbow & Gill & - & - & - & - & - & - & - & & mostly normal, three fish with aneurisms +2 \\
\hline $\begin{array}{l}P P-5 \\
(n=15)\end{array}$ & Rainbow & Liver & 0 & +3.5 & & & & & +3 & & Few fish - hepatomegaly, focal necrosis \\
\hline $\begin{array}{l}\mathrm{PP}-5 \\
(\mathrm{n}=15)\end{array}$ & Rainbow & Kidney & & & & & & & & +3 & $\begin{array}{l}\text { Hypertrophy tubule epithelia; sclerotic } \\
\text { Glomeruli. }\end{array}$ \\
\hline $\begin{array}{l}\mathrm{PP}-5 \\
(\mathrm{n}=15)\end{array}$ & Rainbow & Spleen & & & & & & & & 2.5 & Distinct nodes \\
\hline $\begin{array}{l}P P-5 \\
(n=15)\end{array}$ & Rainbow & G.I. & & & & & & & & & Increased mucous \\
\hline $\begin{array}{l}P P-5 \\
(n=15)\end{array}$ & Rainbow & $\begin{array}{l}\text { Skel. } \\
\text { Muscle }\end{array}$ & & & & & & & & & Mostly normal \\
\hline $\begin{array}{l}\mathrm{PP}-5 \\
(\mathrm{n}=15)\end{array}$ & Rainbow & $\begin{array}{c}\text { General } \\
\text { condition }\end{array}$ & & & Moder & ate changes in & liver, kidney an & nd spleen. Few $\mathrm{fi}$ & fish showed & $d$ increase in bi & le ducts. \\
\hline $\begin{array}{l}\text { ULGP } \\
(n=6)\end{array}$ & Cutthroat & Gill & - & - & - & - & - & - & - & & $\begin{array}{l}\text { Mostly normal, mild scattered fusion of } \\
\text { lamellae. }\end{array}$ \\
\hline $\begin{array}{l}\text { ULGP } \\
(n=6)\end{array}$ & Cutthroat & Liver & +20 & +3 & & +2 & & & +3 & +2 & $\begin{array}{c}\text { Hepatomegally, liquifactive necrosis, inflam. } \\
\text { Diffusely scattered to widespread. }\end{array}$ \\
\hline $\begin{array}{l}\text { ULGP } \\
(n=6)\end{array}$ & Cutthroat & Kidney & & & & & & & & +2.5 & $\begin{array}{l}\text { Regenerating tubules }+2 \text {; sclerotic glomeruli } \\
+3 \text {; erythrophagia }+2 \text {; cont. gen. condition }\end{array}$ \\
\hline $\begin{array}{l}\text { ULGP } \\
(n=6)\end{array}$ & Cutthroat & Spleen & & & & & & & & +2 & \\
\hline
\end{tabular}


Histological analysis (Continued)

\begin{tabular}{|c|c|c|c|c|c|c|c|c|c|c|c|}
\hline $\begin{array}{l}\text { Site ID } \\
\text { (n) }\end{array}$ & Species & Tissue & $\begin{array}{c}\text { Glycogen } \\
\text { Vacuolation }\end{array}$ & $\begin{array}{l}\text { Scattered } \\
\text { Degeneration } \\
\text { Hepatocytes }\end{array}$ & $\begin{array}{c}\text { Focal } \\
\text { Inflammation }\end{array}$ & $\begin{array}{c}\text { Nuclear } \\
\text { Vacuolation }\end{array}$ & $\begin{array}{c}\text { Karryorrhetic } \\
\text { Nuclei }\end{array}$ & Hepatomegally & $\begin{array}{l}\text { Hyper- } \\
\text { cellularity }\end{array}$ & $\begin{array}{l}\text { Melano- } \\
\text { macraphage } \\
\text { Centers }\end{array}$ & Comments \\
\hline $\begin{array}{l}\text { ULGP } \\
(\mathrm{n}=6)\end{array}$ & Cutthroat & G.I. & & & & & & & & & $\begin{array}{l}\text { Little to no feed; migrating lymphocytes } \\
\text { mucosal epithelia }+2.5\end{array}$ \\
\hline $\begin{array}{l}\text { ULGP } \\
(n=6)\end{array}$ & Cutthroat & $\begin{array}{c}\text { Skel. } \\
\text { Muscle }\end{array}$ & & & & & & & & & Mostly normal, scattered degeneration +2 \\
\hline $\begin{array}{l}\text { ULGP } \\
(n=6)\end{array}$ & Cutthroat & $\begin{array}{c}\text { General } \\
\text { comments }\end{array}$ & $\begin{array}{l}\text { Mild accumulc } \\
\text { moderate deg }\end{array}$ & $\begin{array}{l}\text { ation of melanon } \\
\text { generative chang }\end{array}$ & $\begin{array}{l}\text { macraphage ce } \\
\text { ges in tubules. }\end{array}$ & nters in liver; $n$ & mild to moderate & e in kidney, mild $\mathrm{i}$ & in spleen. & Hematipoietic c & centers proliferation moderately severe. Mild \\
\hline $\begin{array}{l}\text { WS-3 } \\
(n=25)\end{array}$ & Brook & Gill & - & - & - & - & - & - & - & & Mostly normal \\
\hline $\begin{array}{l}\text { WS-3 } \\
(n=25)\end{array}$ & Brook & Liver & 0 & +3 & & +2 & & +3 & +2 & & Cystic change +2 ; focal inflammation \\
\hline $\begin{array}{l}\text { WS-3 } \\
(n=25)\end{array}$ & Brook & Kidney & & & & & & & & +3 & $\begin{array}{l}\text { Hyperplasia hematopoietic tissue }+3 \text {, hyper- } \\
\text { trophy, degeneration and sloughing }\end{array}$ \\
\hline $\begin{array}{l}\text { WS-3 } \\
(n=25)\end{array}$ & Brook & Spleen & & & & & & & & +2 & \\
\hline $\begin{array}{l}\text { WS-3 } \\
(n=25)\end{array}$ & Brook & G.I. & & & & & & & & & $\begin{array}{l}\text { Few with feed; migrating lymphocytes } \\
\text { mucosal epithelia }+3\end{array}$ \\
\hline $\begin{array}{l}\text { WS-3 } \\
(n=25)\end{array}$ & Brook & $\begin{array}{c}\text { Skel. } \\
\text { Muscle }\end{array}$ & & & & & & & & & \\
\hline $\begin{array}{l}\text { WS-3 } \\
(n=25)\end{array}$ & Brook & $\begin{array}{c}\text { General } \\
\text { condition }\end{array}$ & In the liver, & bla b & poietic tiss & +3 , hyper- & hy, degenerat & ation and sloughin & ng (early & cification) tubu & ule epithelia +3 , regenerating tubules +3 \\
\hline
\end{tabular}


Lipid Peroxidation

\begin{tabular}{|c|c|c|c|c|c|c|c|c|c|}
\hline Site ID & Date & Species & Sex & $\begin{array}{c}\text { Length } \\
(\mathrm{mm})\end{array}$ & $\begin{array}{c}\text { Weight } \\
\text { (gm) }\end{array}$ & Tissue & Sample ID & $340 \mu \mathrm{m}$ & $360 \mu \mathrm{m}$ \\
\hline PP-1 & $8 / 29 / 01$ & BROOK & $\mathrm{F}$ & 185 & 82 & LIVER & MT-PP1-LIV1 & 243.7 & 144.5 \\
\hline PP-1 & $8 / 29 / 01$ & BROOK & M & 222 & 130 & LIVER & & & \\
\hline PP-1 & $8 / 29 / 01$ & BROOK & $\mathrm{F}$ & 200 & 82 & LIVER & MT-PP1-LIV2 & 270.9 & 167.4 \\
\hline PP-1 & $8 / 29 / 01$ & BROOK & $\mathrm{M}$ & 180 & 63 & LIVER & & & \\
\hline PP-1 & $8 / 29 / 01$ & BROOK & $F$ & 190 & 70 & LIVER & MT-PP1-LIV3 & 231.9 & 134.6 \\
\hline PP-1 & $8 / 29 / 01$ & BROOK & $M$ & 188 & 86 & LIVER & & & \\
\hline PP-1 & $8 / 29 / 01$ & BROOK & $M$ & 168 & 60 & LIVER & & & \\
\hline PP-1 & $8 / 29 / 01$ & BROOK & $\mathrm{F}$ & 206 & 93 & LIVER & MT-PP1-LIV4 & 267 & 154.8 \\
\hline PP-1 & $8 / 29 / 01$ & BROOK & $\mathrm{M}$ & 174 & 51 & LIVER & & & \\
\hline PP-1 & $8 / 29 / 01$ & BROOK & $M$ & 194 & 84 & LIVER & MT-PP1-LIV5 & 310.8 & 213.2 \\
\hline PP-1 & $8 / 29 / 01$ & BROOK & $\mathrm{F}$ & 199 & 77 & LIVER & & & \\
\hline PP-1 & $8 / 29 / 01$ & BROOK & $F$ & 176 & 60 & LIVER & MT-PP1-LIV6 & 205.4 & 110.6 \\
\hline PP-1 & $8 / 29 / 01$ & BROOK & - & 205 & 95 & LIVER & & & \\
\hline PP-1 & $8 / 29 / 01$ & BROOK & $M$ & 200 & 103 & LIVER & MT-PP1-LIV7 & 275.4 & 193.8 \\
\hline PP-1 & $8 / 29 / 01$ & BROOK & $\mathrm{M}$ & 210 & 121 & LIVER & & & \\
\hline PP-1 & $8 / 29 / 01$ & BROOK & $F$ & 185 & 82 & GILL & MT-PP1-GIL1 & 26.10 & 24.73 \\
\hline PP-1 & $8 / 29 / 01$ & BROOK & $\mathrm{M}$ & 222 & 130 & GILL & & & \\
\hline PP-1 & $8 / 29 / 01$ & BROOK & $\mathrm{F}$ & 200 & 82 & GILL & MT-PP1-GIL2 & 16.96 & 15.51 \\
\hline PP-1 & $8 / 29 / 01$ & BROOK & $\mathrm{M}$ & 180 & 63 & GILL & & & \\
\hline PP-1 & $8 / 29 / 01$ & BROOK & $\mathrm{M}$ & 188 & 86 & GILL & MT-PP1-GIL3 & 50.47 & 59.41 \\
\hline PP-1 & 8/29/01 & BROOK & $\mathrm{M}$ & 168 & 60 & GILL & & & \\
\hline PP-1 & $8 / 29 / 01$ & BROOK & $F$ & 206 & 93 & GILL & MT-PP1-GIL4 & 27.51 & 22.56 \\
\hline PP-1 & $8 / 29 / 01$ & BROOK & $\mathrm{M}$ & 174 & 51 & GILL & & & \\
\hline PP-1 & $8 / 29 / 01$ & BROOK & $M$ & 194 & 84 & GILL & MT-PP1-GIL5 & 29.94 & 28.73 \\
\hline PP-1 & $8 / 29 / 01$ & BROOK & $\mathrm{F}$ & 199 & 77 & GILL & & & \\
\hline PP-1 & $8 / 29 / 01$ & BROOK & $F$ & 176 & 60 & GILL & MT-PP1-GIL6 & 124.9 & 159.1 \\
\hline PP-1 & $8 / 29 / 01$ & BROOK & - & 205 & 95 & GILL & & & \\
\hline PP-1 & $8 / 29 / 01$ & BROOK & $M$ & 200 & 103 & GILL & MT-PP1-GIL7 & 32.39 & 33.40 \\
\hline PP-1 & $8 / 29 / 01$ & BROOK & $M$ & 210 & 121 & GILL & & & \\
\hline PP-1 & $8 / 29 / 01$ & BROOK & $F$ & 185 & 82 & CAECA & MT-PP1-PYC1 & 329.6 & 279.5 \\
\hline PP-1 & $8 / 29 / 01$ & BROOK & $\mathrm{M}$ & 222 & 130 & CAECA & & & \\
\hline PP-1 & $8 / 29 / 01$ & BROOK & $F$ & 200 & 82 & CAECA & MT-PP1-PYC2 & 320.8 & 315.3 \\
\hline PP-1 & $8 / 29 / 01$ & BROOK & M & 180 & 63 & CAECA & & & \\
\hline PP-1 & $8 / 29 / 01$ & BROOK & $F$ & 190 & 70 & CAECA & MT-PP1-PYC3 & 314.7 & 273.3 \\
\hline PP-1 & $8 / 29 / 01$ & BROOK & M & 188 & 86 & CAECA & & & \\
\hline PP-1 & 8/29/01 & BROOK & $\mathrm{M}$ & 168 & 60 & CAECA & & & \\
\hline PP-1 & $8 / 29 / 01$ & BROOK & $\mathrm{F}$ & 206 & 93 & CAECA & MT-PP1-PYC4 & 310.8 & 273.3 \\
\hline PP-1 & 8/29/01 & BROOK & $\mathrm{M}$ & 174 & 51 & CAECA & & & \\
\hline PP-1 & $8 / 29 / 01$ & BROOK & $\mathrm{M}$ & 194 & 84 & CAECA & MT-PP1-PYC5 & 370.2 & 299.8 \\
\hline PP-1 & $8 / 29 / 01$ & BROOK & $\mathrm{F}$ & 199 & 77 & CAECA & & & \\
\hline PP-1 & $8 / 29 / 01$ & BROOK & $\mathrm{F}$ & 176 & 60 & CAECA & MT-PP1-PYC6 & 231.2 & 290.5 \\
\hline PP-1 & $8 / 29 / 01$ & BROOK & - & 205 & 95 & CAECA & & & \\
\hline PP-1 & $8 / 29 / 01$ & BROOK & $M$ & 200 & 103 & CAECA & MT-PP1-PYC7 & 329.6 & 238.8 \\
\hline PP-1 & $8 / 29 / 01$ & BROOK & $\mathrm{M}$ & 210 & 121 & CAECA & & & \\
\hline PP-1 & $8 / 29 / 01$ & BROOK & $\mathrm{F}$ & 185 & 82 & FILLET & MT-PP1-FIL1 & 36.76 & 32.03 \\
\hline PP-1 & $8 / 29 / 01$ & BROOK & $\mathrm{M}$ & 222 & 130 & FILLET & & & \\
\hline PP-1 & $8 / 29 / 01$ & BROOK & $\mathrm{F}$ & 200 & 82 & FILLET & MT-PP1-FIL2 & 21.59 & 18.71 \\
\hline PP-1 & 8/29/01 & BROOK & M & 180 & 63 & FILLET & & & \\
\hline PP-1 & $8 / 29 / 01$ & BROOK & $\mathrm{F}$ & 190 & 70 & FILLET & MT-PP1-FIL3 & 15.95 & 12.28 \\
\hline PP-1 & $8 / 29 / 01$ & BROOK & M & 188 & 86 & FILLET & & & \\
\hline PP-1 & $8 / 29 / 01$ & BROOK & $\mathrm{M}$ & 168 & 60 & FILLET & & & \\
\hline PP-1 & $8 / 29 / 01$ & BROOK & $F$ & 206 & 93 & FILLET & MT-PP1-FIL4 & 19.25 & 18.03 \\
\hline PP-1 & $8 / 29 / 01$ & BROOK & M & 174 & 51 & FILLET & & & \\
\hline
\end{tabular}


Lipid Peroxidation (Continued)

\begin{tabular}{|c|c|c|c|c|c|c|c|c|c|}
\hline Site ID & Date & Species & Sex & $\begin{array}{l}\text { Length } \\
\text { (mm) }\end{array}$ & $\begin{array}{l}\text { Weight } \\
\text { (gm) }\end{array}$ & Tissue & Sample ID & $340 \mu \mathrm{m}$ & $360 \mu \mathrm{m}$ \\
\hline PP-1 & $8 / 29 / 01$ & BROOK & M & 194 & 84 & FILLET & MT-PP1-FIL5 & 33.72 & 28.88 \\
\hline PP-1 & $8 / 29 / 01$ & BROOK & $\mathrm{F}$ & 199 & 77 & FILLET & & & \\
\hline PP-1 & $8 / 29 / 01$ & BROOK & $F$ & 176 & 60 & FILLET & MT-PP1-FIL6 & 25.55 & 19.72 \\
\hline PP-1 & $8 / 29 / 01$ & BROOK & - & 205 & 95 & FILLET & & & \\
\hline PP-1 & $8 / 29 / 01$ & BROOK & $M$ & 200 & 103 & FILLET & MT-PP1-FIL7 & 57.73 & 68.38 \\
\hline PP-1 & $8 / 29 / 01$ & BROOK & M & 210 & 121 & FILLET & & & \\
\hline PP-1 & $8 / 29 / 01$ & BROOK & $\mathrm{F}$ & 185 & 82 & WL. FISH & MT-PP1-WF1 & N/A & $\mathrm{N} / \mathrm{A}$ \\
\hline PP-1 & $8 / 29 / 01$ & BROOK & $\mathrm{F}$ & 200 & 82 & WL. FISH & MT-PP1-WF2 & $\mathrm{N} / \mathrm{A}$ & $\mathrm{N} / \mathrm{A}$ \\
\hline PP-1 & $8 / 29 / 01$ & BROOK & $\mathrm{F}$ & 190 & 70 & WL. FISH & MT-PP1-WF3 & $\mathrm{N} / \mathrm{A}$ & $\mathrm{N} / \mathrm{A}$ \\
\hline PP-1 & $8 / 29 / 01$ & BROOK & M & 168 & 60 & WL. FISH & MT-PP1-WF4 & $\mathrm{N} / \mathrm{A}$ & $\mathrm{N} / \mathrm{A}$ \\
\hline PP-1 & $8 / 29 / 01$ & BROOK & $\mathrm{M}$ & 194 & 84 & WL. FISH & MT-PP1-WF5 & $\mathrm{N} / \mathrm{A}$ & $\mathrm{N} / \mathrm{A}$ \\
\hline PP-3 & $8 / 28 / 01$ & BROOK & $F$ & 164 & 43 & LIVER & & & \\
\hline PP-3 & $8 / 28 / 01$ & BROOK & M & 167 & 39 & LIVER & MT-PP3-LIV1 & 187.1 & 142.8 \\
\hline PP-3 & $8 / 28 / 01$ & BROOK & M & 134 & 20 & LIVER & & & \\
\hline PP-3 & $8 / 28 / 01$ & BROOK & $M$ & 167 & 43 & LIVER & & & \\
\hline PP-3 & $8 / 28 / 01$ & BROOK & M & 151 & 32 & LIVER & MT-PP3-LIV2 & 186.6 & 137.7 \\
\hline PP-3 & $8 / 28 / 01$ & BROOK & $\mathrm{F}$ & 139 & 28 & LIVER & & & \\
\hline PP-3 & $8 / 28 / 01$ & BROOK & $\mathrm{F}$ & 140 & 22 & LIVER & & & \\
\hline PP-3 & $8 / 28 / 01$ & BROOK & M & 163 & 39 & LIVER & MT-PP3-LIV3 & 165.7 & 131.1 \\
\hline PP-3 & $8 / 28 / 01$ & BROOK & M & 169 & 46 & LIVER & & & \\
\hline PP-3 & $8 / 28 / 01$ & BROOK & $M$ & 190 & 76 & LIVER & MT-PP3-LIV4 & 240.1 & 154.8 \\
\hline PP-3 & $8 / 28 / 01$ & BROOK & M & 150 & 32 & LIVER & & & \\
\hline PP-3 & $8 / 28 / 01$ & BROOK & $F$ & 153 & 43 & LIVER & & & \\
\hline PP-3 & $8 / 28 / 01$ & BROOK & $F$ & 147 & 28 & LIVER & MT-PP3-LIV5 & 250.1 & 169.5 \\
\hline PP-3 & $8 / 28 / 01$ & BROOK & M & 151 & 29 & LIVER & & & \\
\hline PP-3 & $8 / 28 / 01$ & BROOK & $\mathrm{F}$ & 243 & 140 & LIVER & MT-PP3-LIV6 & 257.1 & 162.7 \\
\hline PP-3 & $8 / 28 / 01$ & RAINBOW & $\mathrm{F}$ & 247 & 140 & LIVER & MT-PP3-LIV7 & 120.4 & 93.10 \\
\hline PP-3 & $8 / 28 / 01$ & RAINBOW & $\mathrm{M}$ & 268 & 194 & LIVER & MT-PP3-LIV8 & 81.32 & 66.44 \\
\hline PP-3 & $8 / 28 / 01$ & RAINBOW & $\mathrm{M}$ & 265 & 176 & LIVER & MT-PP3-LIV9 & 142.0 & 94.63 \\
\hline PP-3 & $8 / 28 / 01$ & RAINBOW & $\mathrm{F}$ & 302 & 222 & LIVER & MT-PP3-LIV10 & 61.59 & 57.07 \\
\hline PP-3 & $8 / 28 / 01$ & RAINBOW & $\mathrm{F}$ & 302 & 258 & LIVER & MT-PP3-LIV11 & 182.4 & 116.4 \\
\hline PP-3 & $8 / 28 / 01$ & BROOK & $F$ & 164 & 43 & $\overline{\text { GILL }}$ & & & \\
\hline PP-3 & $8 / 28 / 01$ & BROOK & M & 167 & 39 & GILL & MT-PP3-GIL1 & 22.21 & 20.68 \\
\hline PP-3 & $8 / 28 / 01$ & BROOK & M & 134 & 20 & GILL & & & \\
\hline PP-3 & $8 / 28 / 01$ & BROOK & M & 167 & 43 & GILL & & & \\
\hline PP-3 & $8 / 28 / 01$ & BROOK & M & 151 & 32 & GILL & MT-PP3-GIL2 & 25.74 & 23.77 \\
\hline PP-3 & $8 / 28 / 01$ & BROOK & $\mathrm{F}$ & 139 & 28 & GILL & & & \\
\hline PP-3 & $8 / 28 / 01$ & BROOK & $\mathrm{F}$ & 140 & 22 & GILL & & & \\
\hline PP-3 & $8 / 28 / 01$ & BROOK & M & 163 & 39 & GILL & MT-PP3-GIL3 & 36.01 & 33.05 \\
\hline PP-3 & $8 / 28 / 01$ & BROOK & M & 169 & 46 & GILL & & & \\
\hline PP-3 & $8 / 28 / 01$ & BROOK & M & 190 & 76 & GILL & MT-PP3-GIL4 & 20.25 & 19.88 \\
\hline PP-3 & $8 / 28 / 01$ & BROOK & M & 150 & 32 & GILL & & & \\
\hline PP-3 & $8 / 28 / 01$ & BROOK & $F$ & 153 & 43 & GILL & & & \\
\hline PP-3 & $8 / 28 / 01$ & BROOK & $F$ & 147 & 28 & GILL & MT-PP3-GIL5 & 42.87 & 49.32 \\
\hline PP-3 & $8 / 28 / 01$ & BROOK & M & 151 & 29 & GILL & & & \\
\hline PP-3 & $8 / 28 / 01$ & BROOK & $\mathrm{F}$ & 243 & 140 & GILL & MT-PP3-GIL6 & 36.31 & 32.46 \\
\hline PP-3 & $8 / 28 / 01$ & RAINBOW & $\mathrm{F}$ & 247 & 140 & GILL & MT-PP3-GIL7 & 49.12 & 45.24 \\
\hline PP-3 & $8 / 28 / 01$ & RAINBOW & M & 268 & 194 & GILL & MT-PP3-GIL8 & 22.85 & 20.72 \\
\hline PP-3 & $8 / 28 / 01$ & RAINBOW & $M$ & 265 & 176 & GILL & MT-PP3-GIL9 & 24.20 & 23.10 \\
\hline PP-3 & $8 / 28 / 01$ & RAINBOW & $\mathrm{F}$ & 302 & 222 & GILL & MT-PP3-GIL10 & 30.17 & 29.33 \\
\hline PP-3 & $8 / 28 / 01$ & RAINBOW & $\mathrm{F}$ & 302 & 258 & GILL & MT-PP3-GIL11 & 24.94 & 22.23 \\
\hline PP-3 & $8 / 28 / 01$ & BROOK & $\mathrm{F}$ & 164 & 43 & CAECA & & & \\
\hline PP-3 & $8 / 28 / 01$ & BROOK & M & 167 & 39 & CAECA & MT-PP3-PYC1 & 333.7 & 294.8 \\
\hline
\end{tabular}


Lipid Peroxidation (Continued)

\begin{tabular}{|c|c|c|c|c|c|c|c|c|c|}
\hline Site ID & Date & Species & Sex & Length & Weight & Tissue & Sample ID & $340 \mu \mathrm{m}$ & $360 \mu \mathrm{m}$ \\
\hline PP-3 & $8 / 28 / 01$ & BROOK & $\mathrm{M}$ & 134 & 20 & CAECA & & & \\
\hline PP-3 & $8 / 28 / 01$ & BROOK & $M$ & 167 & 43 & CAECA & & & \\
\hline PP-3 & $8 / 28 / 01$ & BROOK & $M$ & 151 & 32 & CAECA & MT-PP3-PYC2 & 333.7 & 294.8 \\
\hline PP-3 & $8 / 28 / 01$ & BROOK & $\mathrm{F}$ & 139 & 28 & CAECA & & & \\
\hline PP-3 & $8 / 28 / 01$ & BROOK & $F$ & 140 & 22 & CAECA & & & \\
\hline PP-3 & $8 / 28 / 01$ & BROOK & $M$ & 163 & 39 & CAECA & MT-PP3-PYC3 & 357.4 & 295.3 \\
\hline PP-3 & $8 / 28 / 01$ & BROOK & $\mathrm{M}$ & 169 & 46 & CAECA & & & \\
\hline PP-3 & $8 / 28 / 01$ & BROOK & $M$ & 190 & 76 & CAECA & MT-PP3-PYC4 & 361.5 & 290.9 \\
\hline PP-3 & $8 / 28 / 01$ & BROOK & $\mathrm{M}$ & 150 & 32 & CAECA & & & \\
\hline PP-3 & $8 / 28 / 01$ & BROOK & $F$ & 153 & 43 & CAECA & & & \\
\hline PP-3 & $8 / 28 / 01$ & BROOK & $F$ & 147 & 28 & CAECA & MT-PP3-PYC5 & 328.7 & 264.1 \\
\hline PP-3 & $8 / 28 / 01$ & BROOK & $\mathrm{M}$ & 151 & 29 & CAECA & & & \\
\hline PP-3 & $8 / 28 / 01$ & BROOK & $\mathrm{F}$ & 243 & 140 & CAECA & MT-PP3-PYC6 & 290.7 & 242.8 \\
\hline PP-3 & $8 / 28 / 01$ & RAINBOW & $\mathrm{F}$ & 247 & 140 & CAECA & MT-PP3-PYC7 & 55.06 & 79.45 \\
\hline PP-3 & $8 / 28 / 01$ & RAINBOW & $\mathrm{M}$ & 268 & 194 & CAECA & MT-PP3-PYC8 & 23.95 & 38.06 \\
\hline PP-3 & $8 / 28 / 01$ & RAINBOW & $\mathrm{M}$ & 265 & 176 & CAECA & MT-PP3-PYC9 & 64.16 & 38.98 \\
\hline PP-3 & $8 / 28 / 01$ & RAINBOW & $\mathrm{F}$ & 302 & 222 & CAECA & MT-PP3-PYC10 & 114.9 & 133.4 \\
\hline PP-3 & $8 / 28 / 01$ & RAINBOW & $\mathrm{F}$ & 302 & 258 & CAECA & MT-PP3-PYC11 & 147.0 & 117.7 \\
\hline PP-3 & $8 / 28 / 01$ & BROOK & $F$ & 164 & 43 & FILLET & & & \\
\hline PP-3 & $8 / 28 / 01$ & BROOK & $M$ & 167 & 39 & FILLET & MT-PP-3-FIL1 & 19.95 & 16.82 \\
\hline PP-3 & $8 / 28 / 01$ & BROOK & $\mathrm{M}$ & 134 & 20 & FILLET & & & \\
\hline PP-3 & $8 / 28 / 01$ & BROOK & $M$ & 167 & 43 & FILLET & & & \\
\hline PP-3 & $8 / 28 / 01$ & BROOK & $\mathrm{M}$ & 151 & 32 & FILLET & MT-PP-3-FIL2 & 12.80 & 11.04 \\
\hline $\mathrm{PP}^{*} 3$ & $8 / 28 / 01$ & BROOK & $\mathrm{F}$ & 139 & 28 & FILLET & & & \\
\hline PP-3 & $8 / 28 / 01$ & BROOK & $\mathrm{F}$ & 140 & 22 & FILLET & & & \\
\hline PP-3 & $8 / 28 / 01$ & BROOK & $\mathrm{M}$ & 163 & 39 & FILLET & MT-PP3-FIL3 & 13.37 & 11.78 \\
\hline PP-3 & $8 / 28 / 01$ & BROOK & $\mathrm{M}$ & 169 & 46 & FILLET & & & \\
\hline PP-3 & $8 / 28 / 01$ & BROOK & $\mathrm{M}$ & 190 & 76 & FILLET & MT-PP3-FIL4 & 19.79 & 17.52 \\
\hline PP-3 & $8 / 28 / 01$ & BROOK & $\mathrm{M}$ & 150 & 32 & FILLET & & & \\
\hline PP-3 & $8 / 28 / 01$ & BROOK & $\mathrm{F}$ & 153 & 43 & FILLET & & & \\
\hline PP-3 & $8 / 28 / 01$ & BROOK & $\mathrm{F}$ & 147 & 28 & FILLET & MT-PP3-FIL5 & 17.5 & 16.98 \\
\hline PP-3 & $8 / 28 / 01$ & BROOK & M & 151 & 29 & FILLET & & & \\
\hline PP-3 & $8 / 28 / 01$ & BROOK & $\mathrm{F}$ & 243 & 140 & FILLET & MT-PP3-FIL6 & 30.55 & 31.17 \\
\hline PP-3 & $8 / 28 / 01$ & RAINBOW & $\mathrm{F}$ & 247 & 140 & FILLET & MT-PP3-FIL7 & 16.64 & 16.19 \\
\hline PP-3 & $8 / 28 / 01$ & RAINBOW & $\mathrm{M}$ & 268 & 194 & FILLET & MT-PP3-FIL8 & 15.33 & 15.14 \\
\hline PP-3 & $8 / 28 / 01$ & RAINBOW & $\mathrm{M}$ & 265 & 176 & FILLET & MT-PP3-FIL9 & 23.03 & 24.14 \\
\hline PP-3 & $8 / 28 / 01$ & RAINBOW & $\mathrm{F}$ & 302 & 222 & FILLET & MT-PP3-FIL10 & 20.35 & 16.73 \\
\hline PP-3 & $8 / 28 / 01$ & RAINBOW & $\mathrm{F}$ & 302 & 258 & FILLET & MT-PP3-FIL11 & 18.82 & 16.63 \\
\hline PP-3 & $8 / 28 / 01$ & BROOK & - & 92 & 8 & WL. FISH & MT-PP3-WF1 & N/A & $\mathrm{N} / \mathrm{A}$ \\
\hline PP-3 & $8 / 28 / 01$ & BROOK & - & 92 & 7 & WL. FISH & MT-PP3-WF2 & $\mathrm{N} / \mathrm{A}$ & $\mathrm{N} / \mathrm{A}$ \\
\hline PP-3 & $8 / 28 / 01$ & BROOK & - & 90 & 6 & WL. FISH & MT-PP3-WF3 & $\mathrm{N} / \mathrm{A}$ & $\mathrm{N} / \mathrm{A}$ \\
\hline PP-3 & $8 / 28 / 01$ & BROOK & - & 95 & 9 & WL. FISH & MT-PP3-WF4 & $\mathrm{N} / \mathrm{A}$ & $\mathrm{N} / \mathrm{A}$ \\
\hline PP-3 & $8 / 28 / 01$ & BROOK & - & 94 & 8 & WL. FISH & MT-PP3-WF5 & N/A & $\mathrm{N} / \mathrm{A}$ \\
\hline PP-3 & $8 / 28 / 01$ & RAINBOW & - & 139 & 24 & WL. FISH & MT-PP3-WF6 & $\mathrm{N} / \mathrm{A}$ & $\mathrm{N} / \mathrm{A}$ \\
\hline PP-3 & $8 / 28 / 01$ & RAINBOW & - & 125 & 17 & WL. FISH & MT-PP3-WF7 & $\mathrm{N} / \mathrm{A}$ & $\mathrm{N} / \mathrm{A}$ \\
\hline PP-3 & $8 / 28 / 01$ & RAINBOW & - & 165 & 41 & WL. FISH & MT-PP3-WF8 & $\mathrm{N} / \mathrm{A}$ & $\mathrm{N} / \mathrm{A}$ \\
\hline PP-3 & $8 / 28 / 01$ & RAINBOW & - & 133 & 22 & WL. FISH & MT-PP3-WF9 & $\mathrm{N} / \mathrm{A}$ & $\mathrm{N} / \mathrm{A}$ \\
\hline PP-3 & $8 / 28 / 01$ & RAINBOW & - & 213 & 96 & WL. FISH & MT-PP3-WF10 & $\mathrm{N} / \mathrm{A}$ & $\mathrm{N} / \mathrm{A}$ \\
\hline PP-5 & $8 / 28 / 01$ & RAINBOW & $M$ & 153 & 37 & LIVER & & & \\
\hline PP-5 & $8 / 28 / 01$ & RAINBOW & $\mathrm{M}$ & 167 & 42 & LIVER & MT-PP5-LIV1 & 190.3 & 132.2 \\
\hline PP-5 & $8 / 28 / 01$ & RAINBOW & $\mathrm{M}$ & 154 & 36 & LIVER & & & \\
\hline PP-5 & $8 / 28 / 01$ & RAINBOW & $\mathrm{F}$ & 155 & 34 & LIVER & MT-PP5-LIV2 & 158.8 & 104.1 \\
\hline
\end{tabular}


Lipid Peroxidation (Continued)

\begin{tabular}{|c|c|c|c|c|c|c|c|c|c|}
\hline Site ID & Date & Species & Sex & Length & Weight & Tissue & Sample ID & $340 \mu \mathrm{m}$ & $360 \mu \mathrm{m}$ \\
\hline PP-5 & $8 / 28 / 01$ & RAINBOW & $\mathrm{M}$ & 220 & 106 & LIVER & & & \\
\hline PP-5 & $8 / 28 / 01$ & RAINBOW & M & 222 & 99 & LIVER & MT-PP5-LIV3 & 161.3 & 99.42 \\
\hline PP-5 & 8/28/01 & RAINBOW & $\mathrm{F}$ & 161 & 43 & LIVER & & & \\
\hline PP-5 & $8 / 28 / 01$ & RAINBOW & $M$ & 195 & 76 & LIVER & MT-PP5-LIV4 & 170.3 & 105.7 \\
\hline PP-5 & 8/28/01 & RAINBOW & $\mathrm{F}$ & 167 & 46 & LIVER & & & \\
\hline PP-5 & $8 / 28 / 01$ & RAINBOW & $M$ & 185 & 64 & LIVER & MT-PP5-LIV5 & 156.1 & 100.7 \\
\hline PP-5 & 8/28/01 & RAINBOW & $\mathrm{F}$ & 174 & 49 & LIVER & & & \\
\hline PP-5 & $8 / 28 / 01$ & RAINBOW & $M$ & 173 & 50 & LIVER & MT-PP5-LIV6 & 128.5 & 82.68 \\
\hline PP-5 & 8/28/01 & RAINBOW & $\mathrm{M}$ & 154 & 60 & LIVER & & & \\
\hline PP-5 & $8 / 28 / 01$ & RAINBOW & $M$ & 182 & 60 & LIVER & MT-PP5-LIV7 & 180.9 & 128.3 \\
\hline PP-5 & 8/28/01 & RAINBOW & $\mathrm{F}$ & 168 & 46 & LIVER & & & \\
\hline PP-5 & $8 / 28 / 01$ & RAINBOW & $M$ & 153 & 37 & "GILL & & & \\
\hline PP-5 & $8 / 28 / 01$ & RAINBOW & $\mathrm{M}$ & 167 & 42 & GILL & MT-PP5-GIL1 & 20.35 & 19.27 \\
\hline PP-5 & $8 / 28 / 01$ & RAINBOW & $\mathrm{M}$ & 154 & 36 & GILL & & & \\
\hline PP-5 & $8 / 28 / 01$ & RAINBOW & $\mathrm{F}$ & 155 & 34 & GILL & MT-PP5-GIL2 & 47.05 & 43.47 \\
\hline PP-5 & 8/28/01 & RAINBOW & $\mathrm{M}$ & 220 & 106 & GILL & & & \\
\hline PP-5 & $8 / 28 / 01$ & RAINBOW & $M$ & 222 & 99 & GILL & MT-PP5-GIL3 & 26.03 & 24.69 \\
\hline PP-5 & 8/28/01 & RAINBOW & $\mathrm{F}$ & 161 & 43 & GILL & & & \\
\hline PP-5 & $8 / 28 / 01$ & RAINBOW & $M$ & 195 & 76 & GILL & MT-PP5-GIL4 & 26.42 & 24.73 \\
\hline PP-5 & 8/28/01 & RAINBOW & $\mathrm{F}$ & 167 & 46 & GILL & & & \\
\hline PP-5 & $8 / 28 / 01$ & RAINBOW & $M$ & 185 & 64 & GILL & MT-PP5-GIL5 & 18.06 & 17.17 \\
\hline PP-5 & 8/28/01 & RAINBOW & $\mathrm{F}$ & 174 & 49 & GILL & & & \\
\hline PP-5 & $8 / 28 / 01$ & RAINBOW & $M$ & 173 & 50 & GILL & MT-PP5-GIL6 & 19.15 & 17.66 \\
\hline PP-5 & 8/28/01 & RAINBOW & $\mathrm{M}$ & 154 & 60 & GILL & & & \\
\hline PP-5 & $8 / 28 / 01$ & RAINBOW & $M$ & 182 & 60 & GILL & MT-PP5-LIV7 & 25.01 & 23.19 \\
\hline PP-5 & 8/28/01 & RAINBOW & $\mathrm{F}$ & 168 & 46 & GILL & & & \\
\hline PP-5 & $8 / 28 / 01$ & RAINBOW & $M$ & 153 & 37 & CAECA & & & \\
\hline PP-5 & 8/28/01 & RAINBOW & M & 167 & 42 & CAECA & MT-PP5-PYC1 & 201.0 & 125.1 \\
\hline PP-5 & 8/28/01 & RAINBOW & M & 154 & 36 & CAECA & & & \\
\hline PP-5 & $8 / 28 / 01$ & RAINBOW & $F$ & 155 & 34 & CAECA & MT-PP5-PYC2 & 156.1 & 110.5 \\
\hline PP-5 & 8/28/01 & RAINBOW & $\mathrm{M}$ & 220 & 106 & CAECA & & & \\
\hline PP-5 & $8 / 28 / 01$ & RAINBOW & $M$ & 222 & 99 & CAECA & MT-PP5-PYC3 & 216.4 & 129.2 \\
\hline PP-5 & 8/28/01 & RAINBOW & $\mathrm{F}$ & 161 & 43 & CAECA & & & \\
\hline PP-5 & $8 / 28 / 01$ & RAINBOW & $M$ & 195 & 76 & CAECA & MT-PP5-PYC4 & $\mathrm{N} / \mathrm{A}$ & $\mathrm{N} / \mathrm{A}$ \\
\hline PP-5 & $8 / 28 / 01$ & RAINBOW & $\mathrm{F}$ & 167 & 46 & CAECA & & & \\
\hline PP-5 & $8 / 28 / 01$ & RAINBOW & $M$ & 185 & 64 & CAECA & MT-PP5-PYC5 & 209.1 & 129.8 \\
\hline PP-5 & 8/28/01 & RAINBOW & $\mathrm{F}$ & 174 & 49 & CAECA & & & \\
\hline PP-5 & $8 / 28 / 01$ & RAINBOW & $M$ & 173 & 50 & CAECA & MT-PP5-PYC6 & 151.9 & 91.27 \\
\hline PP-5 & 8/28/01 & RAINBOW & $\mathrm{M}$ & 154 & 60 & CAECA & & & \\
\hline PP-5 & $8 / 28 / 01$ & RAINBOW & $M$ & 182 & 60 & CAECA & MT-PP5-PYC7 & 201.7 & 119.4 \\
\hline PP-5 & 8/28/01 & RAINBOW & $\mathrm{F}$ & 168 & 46 & CAECA & & & \\
\hline PP-5 & $8 / 28 / 01$ & RAINBOW & $M$ & 153 & 37 & FILLET & & & \\
\hline PP-5 & 8/28/01 & RAINBOW & $M$ & 167 & 42 & FILLET & MT-PP5-FIL1 & 21.82 & 20.8 \\
\hline PP-5 & $8 / 28 / 01$ & RAINBOW & M & 154 & 36 & FILLET & & & \\
\hline PP-5 & $8 / 28 / 01$ & RAINBOW & $F$ & 155 & 34 & FILLET & MT-PP5-FIL2 & 15.71 & 14.24 \\
\hline PP-5 & 8/28/01 & RAINBOW & $\mathrm{M}$ & 220 & 106 & FILLET & & & \\
\hline PP-5 & $8 / 28 / 01$ & RAINBOW & $M$ & 222 & 99 & FILLET & MT-PP5-FIL3 & 27.34 & 23.19 \\
\hline PP-5 & 8/28/01 & RAINBOW & $\mathrm{F}$ & 161 & 43 & FILLET & & & \\
\hline PP-5 & $8 / 28 / 01$ & RAINBOW & $M$ & 195 & 76 & FILLET & MT-PP5-FIL4 & 17.08 & 14.67 \\
\hline PP-5 & $8 / 28 / 01$ & RAINBOW & $\mathrm{F}$ & 167 & 46 & FILLET & & & \\
\hline
\end{tabular}


Lipid Peroxidation (Continued)

\begin{tabular}{|c|c|c|c|c|c|c|c|c|c|}
\hline Site ID & Date & Species & Sex & Length & Weight & Tissue & Sample ID & $340 \mu \mathrm{m}$ & $360 \mu \mathrm{m}$ \\
\hline PP-5 & $8 / 28 / 01$ & RAINBOW & $M$ & 185 & 64 & FILLET & MT-PP5-FIL5 & 20.72 & 19.44 \\
\hline PP-5 & 8/28/01 & RAINBOW & $\mathrm{F}$ & 174 & 49 & FILLET & & & \\
\hline PP-5 & $8 / 28 / 01$ & RAINBOW & $\mathrm{M}$ & 173 & 50 & FILLET & MT-PP5-FIL6 & 176.6 & 108.4 \\
\hline PP-5 & $8 / 28 / 01$ & RAINBOW & $\mathrm{M}$ & 154 & 60 & FILLET & & & \\
\hline PP-5 & $8 / 28 / 01$ & RAINBOW & $M$ & 182 & 60 & FILLET & MT-PP5-FIL7 & 18.05 & 16.60 \\
\hline PP-5 & $8 / 28 / 01$ & RAINBOW & $\mathrm{F}$ & 168 & 46 & FILLET & & & \\
\hline PP-5 & $8 / 28 / 01$ & RAINBOW & 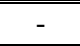 & 153 & 37 & WL. FISH & MT-PP5-WF1 & $\mathrm{N} / \mathrm{A}$ & $\mathrm{N} / \mathrm{A}$ \\
\hline PP-5 & $8 / 28 / 01$ & RAINBOW & - & 167 & 42 & WL. FISH & MT-PP5-WF2 & $\mathrm{N} / \mathrm{A}$ & $\mathrm{N} / \mathrm{A}$ \\
\hline PP-5 & $8 / 28 / 01$ & RAINBOW & - & 154 & 36 & WL. FISH & MT-PP5-WF3 & $\mathrm{N} / \mathrm{A}$ & $\mathrm{N} / \mathrm{A}$ \\
\hline PP-5 & $8 / 28 / 01$ & RAINBOW & - & 155 & 34 & WL. FISH & MT-PP5-WF4 & $\mathrm{N} / \mathrm{A}$ & $\mathrm{N} / \mathrm{A}$ \\
\hline PP-5 & $8 / 28 / 01$ & RAINBOW & - & 220 & 106 & WL. FISH & MT-PP5-WF5 & $\mathrm{N} / \mathrm{A}$ & $\mathrm{N} / \mathrm{A}$ \\
\hline ULGP & $8 / 29 / 01$ & WSC & $\mathrm{M}$ & 420 & 747 & LIVER & MT-ULG-LIV1 & 237.8 & 175.0 \\
\hline ULGP & $8 / 29 / 01$ & WSC & $\mathrm{M}$ & 302 & 248 & LIVER & MT-ULG-LIV2 & 181.0 & 116.8 \\
\hline ULGP & $8 / 29 / 01$ & WSC & $M$ & 312 & 270 & LIVER & MT-ULG-LIV3 & 527.9 & 250.3 \\
\hline ULGP & $8 / 29 / 01$ & WSC & $\mathrm{M}$ & 294 & 253 & LIVER & MT-ULG-LIV4 & 351.4 & 196.2 \\
\hline ULGP & $8 / 29 / 01$ & WSC & $\mathrm{M}$ & 263 & 174 & LIVER & MT-ULG-LIV5 & 364.5 & 211.8 \\
\hline ULGP & $8 / 29 / 01$ & WSC & $\mathrm{M}$ & 260 & 177 & LIVER & MT-ULG-LIV6 & 352.5 & 194.8 \\
\hline ULGP & $8 / 29 / 01$ & WSC & $\mathrm{M}$ & 420 & 747 & GILL & MT-ULG-LIV1 & 68.64 & 82.25 \\
\hline ULGP & $8 / 29 / 01$ & WSC & $\mathrm{M}$ & 302 & 248 & GILL & MT-ULG-LIV2 & 59.26 & 54.72 \\
\hline ULGP & $8 / 29 / 01$ & WSC & $\mathrm{M}$ & 312 & 270 & GILL & MT-ULG-LIV3 & 45.34 & 40.43 \\
\hline ULGP & $8 / 29 / 01$ & WSC & $\mathrm{M}$ & 294 & 253 & GILL & MT-ULG-LIV4 & 25.84 & 25.70 \\
\hline ULGP & $8 / 29 / 01$ & WSC & $\mathrm{M}$ & 263 & 174 & GILL & MT-ULG-LIV5 & 20.87 & 19.53 \\
\hline ULGP & $8 / 29 / 01$ & WSC & $\mathrm{M}$ & 260 & 177 & GILL & MT-ULG-LIV6 & 30.29 & 27.98 \\
\hline ULGP & $8 / 29 / 01$ & WSC & $\mathrm{M}$ & 420 & 747 & CAECA & MT-ULG-PYC1 & 396.8 & 188.4 \\
\hline ULGP & $8 / 29 / 01$ & WSC & $M$ & 302 & 248 & CAECA & MT-ULG-PYC2 & 257.5 & 153.2 \\
\hline ULGP & $8 / 29 / 01$ & WSC & $\mathrm{M}$ & 312 & 270 & CAECA & MT-ULG-PYC3 & 502.7 & 257.8 \\
\hline ULGP & $8 / 29 / 01$ & WSC & $\mathrm{M}$ & 294 & 253 & CAECA & MT-ULG-PYC4 & 670.6 & 276.1 \\
\hline ULGP & $8 / 29 / 01$ & WSC & $\mathrm{M}$ & 263 & 174 & CAECA & MT-ULG-PYC5 & 598.4 & 265.0 \\
\hline ULGP & $8 / 29 / 01$ & WSC & $\mathrm{M}$ & 260 & 177 & CAECA & MT-ULG-PYC6 & 546.0 & 288.2 \\
\hline ULGP & $8 / 29 / 01$ & WSC & $\mathrm{M}$ & 420 & 747 & FILLET & MT-ULG-FIL1 & 51.12 & 57.20 \\
\hline ULGP & $8 / 29 / 01$ & WSC & $\mathrm{M}$ & 302 & 248 & FILLET & MT-ULG-FIL2 & 30.61 & 23.18 \\
\hline ULGP & 8/29/01 & WSC & $\mathrm{M}$ & 312 & 270 & FILLET & MT-ULG-FIL3 & 11.88 & 10.82 \\
\hline ULGP & $8 / 29 / 01$ & WSC & $M$ & 294 & 253 & FILLET & MT-ULG-FIL4 & 17.09 & 14.60 \\
\hline ULGP & $8 / 29 / 01$ & WSC & $\mathrm{M}$ & 263 & 174 & FILLET & MT-ULG-FIL5 & 32.16 & 30.48 \\
\hline ULGP & $8 / 29 / 01$ & WSC & $\mathrm{M}$ & 260 & 177 & FILLET & MT-ULG-FIL6 & 15.79 & 13.02 \\
\hline ULGP & $8 / 29 / 01$ & WSC & - & 186 & 58 & WL. FISH & MT-ULG-WF 1 & $\mathrm{~N} / \mathrm{A}$ & $\mathrm{N} / \mathrm{A}$ \\
\hline ULGP & $8 / 29 / 01$ & WSC & - & 313 & 277 & WL. FISH & MT-ULG-WF 2 & N/A & $\mathrm{N} / \mathrm{A}$ \\
\hline ULGP & $8 / 29 / 01$ & WSC & - & 370 & 422 & WL. FISH & MT-ULG-WF 3 & $\mathrm{~N} / \mathrm{A}$ & $\mathrm{N} / \mathrm{A}$ \\
\hline WS-3 & $8 / 27 / 01$ & BROOK & $\mathrm{M}$ & 127 & 23 & LIVER & & & \\
\hline WS-3 & $8 / 27 / 01$ & BROOK & - & 93 & 10 & LIVER & & & \\
\hline WS-3 & $8 / 27 / 01$ & BROOK & $F$ & 111 & 12 & LIVER & MT-WS3-LIV1 & 256.5 & 176.5 \\
\hline WS-3 & $8 / 27 / 01$ & BROOK & $\mathrm{F}$ & 114 & 15 & LIVER & & & \\
\hline WS-3 & $8 / 27 / 01$ & BROOK & - & 100 & 9 & LIVER & & & \\
\hline WS-3 & $8 / 27 / 01$ & BROOK & $\mathrm{F}$ & 114 & 17 & LIVER & & & \\
\hline WS-3 & $8 / 27 / 01$ & BROOK & $F$ & 142 & 28 & LIVER & & & \\
\hline WS-3 & $8 / 27 / 01$ & BROOK & $\mathrm{M}$ & 115 & 14 & LIVER & & & \\
\hline WS-3 & $8 / 27 / 01$ & BROOK & - & 97 & 8 & LIVER & MT-WS3-LIV2 & 264.7 & 166.3 \\
\hline WS-3 & $8 / 27 / 01$ & BROOK & M & 110 & 14 & LIVER & & & \\
\hline WS-3 & $8 / 27 / 01$ & BROOK & M & 112 & 13 & LIVER & & & \\
\hline WS-3 & $8 / 27 / 01$ & BROOK & - & 105 & 9 & LIVER & & & \\
\hline WS-3 & $8 / 27 / 01$ & BROOK & $\mathrm{M}$ & 120 & 19 & LIVER & & & \\
\hline
\end{tabular}


Lipid Peroxidation (Continued)

\begin{tabular}{|c|c|c|c|c|c|c|c|c|c|}
\hline Site ID & Date & Species & Sex & Length & Weight & Tissue & Sample ID & $340 \mu \mathrm{m}$ & $360 \mu \mathrm{m}$ \\
\hline WS-3 & $8 / 27 / 01$ & BROOK & $M$ & 132 & 26 & LIVER & & & \\
\hline WS-3 & 8/27/01 & BROOK & $\mathrm{F}$ & 100 & 9 & LIVER & MT-WS3-LIV3 & 247.4 & 174.4 \\
\hline WS-3 & $8 / 27 / 01$ & BROOK & - & 105 & 9 & LIVER & & & \\
\hline WS-3 & $8 / 27 / 01$ & BROOK & M & 102 & 10 & LIVER & & & \\
\hline WS-3 & $8 / 27 / 01$ & BROOK & - & 108 & 12 & LIVER & & & \\
\hline WS-3 & $8 / 27 / 01$ & BROOK & - & 111 & 13 & LIVER & & & \\
\hline WS-3 & $8 / 27 / 01$ & BROOK & M & 125 & 20 & LIVER & & & \\
\hline WS-3 & $8 / 27 / 01$ & BROOK & $\mathrm{M}$ & 112 & 12 & LIVER & MT-WS3-LIV4 & 197.2 & 160.2 \\
\hline WS-3 & $8 / 27 / 01$ & BROOK & - & 109 & 10 & LIVER & & & \\
\hline WS-3 & $8 / 27 / 01$ & BROOK & M & 110 & 13 & LIVER & & & \\
\hline WS-3 & $8 / 27 / 01$ & BROOK & - & 105 & 9 & LIVER & & & \\
\hline WS-3 & $8 / 27 / 01$ & BROOK & - & 98 & 9 & LIVER & & & \\
\hline WS-3 & $8 / 27 / 01$ & BROOK & $\bar{M}$ & 127 & 23 & "GILL & & & \\
\hline WS-3 & $8 / 27 / 01$ & BROOK & - & 93 & 10 & GILL & & & \\
\hline WS-3 & $8 / 27 / 01$ & BROOK & $\mathrm{F}$ & 111 & 12 & GILL & MT-WS3-GILL1 & 45.71 & 46.79 \\
\hline WS-3 & $8 / 27 / 01$ & BROOK & $\mathrm{F}$ & 114 & 15 & GILL & & & \\
\hline WS-3 & $8 / 27 / 01$ & BROOK & - & 100 & 9 & GILL & & & \\
\hline WS-3 & $8 / 27 / 01$ & BROOK & $\mathrm{F}$ & 114 & 17 & GILL & & & \\
\hline WS-3 & $8 / 27 / 01$ & BROOK & $F$ & 142 & 28 & GILL & & & \\
\hline WS-3 & $8 / 27 / 01$ & BROOK & $\mathrm{M}$ & 115 & 14 & GILL & & & \\
\hline WS-3 & $8 / 27 / 01$ & BROOK & - & 97 & 8 & GILL & MT-WS3-GIL2 & 35.36 & 32.83 \\
\hline WS-3 & $8 / 27 / 01$ & BROOK & M & 110 & 14 & GILL & & & \\
\hline WS-3 & $8 / 27 / 01$ & BROOK & M & 112 & 13 & GILL & & & \\
\hline WS-3 & $8 / 27 / 01$ & BROOK & - & 105 & 9 & GILL & & & \\
\hline WS-3 & $8 / 27 / 01$ & BROOK & $M$ & 120 & 19 & GILL & & & \\
\hline WS-3 & $8 / 27 / 01$ & BROOK & $M$ & 132 & 26 & GILL & & & \\
\hline WS-3 & $8 / 27 / 01$ & BROOK & $F$ & 100 & 9 & GILL & MT-WS3-GIL3 & 31.78 & 29.96 \\
\hline WS-3 & $8 / 27 / 01$ & BROOK & - & 105 & 9 & GILL & & & \\
\hline WS-3 & $8 / 27 / 01$ & BROOK & M & 102 & 10 & GILL & & & \\
\hline WS-3 & $8 / 27 / 01$ & BROOK & - & 108 & 12 & GILL & & & \\
\hline WS-3 & $8 / 27 / 01$ & BROOK & - & 111 & 13 & GILL & & & \\
\hline WS-3 & $8 / 27 / 01$ & BROOK & M & 125 & 20 & GILL & & & \\
\hline WS-3 & $8 / 27 / 01$ & BROOK & M & 112 & 12 & GILL & MT-WS3-GIL4 & 14.11 & 12.90 \\
\hline WS-3 & $8 / 27 / 01$ & BROOK & - & 109 & 10 & GILL & & & \\
\hline WS-3 & $8 / 27 / 01$ & BROOK & M & 110 & 13 & GILL & & & \\
\hline WS-3 & $8 / 27 / 01$ & BROOK & - & 105 & 9 & GILL & & & \\
\hline WS-3 & $8 / 27 / 01$ & BROOK & - & 98 & 9 & GILL & & & \\
\hline WS-3 & $8 / 27 / 01$ & BROOK & $\bar{M}$ & 127 & 23 & CAECA & & & \\
\hline WS-3 & $8 / 27 / 01$ & BROOK & - & 93 & 10 & CAECA & & & \\
\hline WS-3 & $8 / 27 / 01$ & BROOK & $\mathrm{F}$ & 111 & 12 & CAECA & MT-WS3-PYC1 & 314.9 & 188.3 \\
\hline WS-3 & $8 / 27 / 01$ & BROOK & $F$ & 114 & 15 & CAECA & & & \\
\hline WS-3 & $8 / 27 / 01$ & BROOK & - & 100 & 9 & CAECA & & & \\
\hline WS-3 & $8 / 27 / 01$ & BROOK & $\mathrm{F}$ & 114 & 17 & CAECA & & & \\
\hline WS-3 & $8 / 27 / 01$ & BROOK & $F$ & 142 & 28 & CAECA & & & \\
\hline WS-3 & $8 / 27 / 01$ & BROOK & $\mathrm{M}$ & 115 & 14 & CAECA & & & \\
\hline WS-3 & $8 / 27 / 01$ & BROOK & - & 97 & 8 & CAECA & MT-WS3-PYC2 & 295.8 & 183.7 \\
\hline WS-3 & $8 / 27 / 01$ & BROOK & M & 110 & 14 & CAECA & & & \\
\hline WS-3 & $8 / 27 / 01$ & BROOK & M & 112 & 13 & CAECA & & & \\
\hline WS-3 & $8 / 27 / 01$ & BROOK & - & 105 & 9 & CAECA & & & \\
\hline WS-3 & $8 / 27 / 01$ & BROOK & $\mathrm{M}$ & 120 & 19 & CAECA & & & \\
\hline WS-3 & $8 / 27 / 01$ & BROOK & M & 132 & 26 & CAECA & & & \\
\hline WS-3 & $8 / 27 / 01$ & BROOK & $\mathrm{F}$ & 100 & 9 & CAECA & MT-WS3-PYC3 & 274.8 & 161.9 \\
\hline WS-3 & $8 / 27 / 01$ & BROOK & - & 105 & 9 & CAECA & & & \\
\hline WS-3 & $8 / 27 / 01$ & BROOK & $M$ & 102 & 10 & CAECA & & & \\
\hline
\end{tabular}


Lipid Peroxidation (Continued)

\begin{tabular}{|c|c|c|c|c|c|c|c|c|c|}
\hline Site ID & Date & Species & Sex & Length & Weight & Tissue & Sample ID & $340 \mu \mathrm{m}$ & $360 \mu \mathrm{m}$ \\
\hline WS-3 & $8 / 27 / 01$ & BROOK & - & 108 & 12 & CAECA & & & \\
\hline WS-3 & $8 / 27 / 01$ & BROOK & - & 111 & 13 & CAECA & & & \\
\hline WS-3 & $8 / 27 / 01$ & BROOK & $M$ & 125 & 20 & CAECA & & & \\
\hline WS-3 & $8 / 27 / 01$ & BROOK & $M$ & 112 & 12 & CAECA & MT-WS3- PYC4 & 343.0 & 219.5 \\
\hline WS-3 & $8 / 27 / 01$ & BROOK & - & 109 & 10 & CAECA & & & \\
\hline WS-3 & $8 / 27 / 01$ & BROOK & $M$ & 110 & 13 & CAECA & & & \\
\hline WS-3 & $8 / 27 / 01$ & BROOK & - & 105 & 9 & CAECA & & & \\
\hline WS-3 & $8 / 27 / 01$ & BROOK & - & 98 & 9 & CAECA & & & \\
\hline WS-3 & $8 / 27 / 01$ & BROOK & $\bar{M}$ & 127 & 23 & FILLET & & & \\
\hline WS-3 & $8 / 27 / 01$ & BROOK & - & 93 & 10 & FILLET & & & \\
\hline WS-3 & $8 / 27 / 01$ & BROOK & $\mathrm{F}$ & 111 & 12 & FILLET & MT-WS3-FIL1 & 24.83 & 18.32 \\
\hline WS-3 & $8 / 27 / 01$ & BROOK & $\mathrm{F}$ & 114 & 15 & FILLET & & & \\
\hline WS-3 & 8/27/01 & BROOK & - & 100 & 9 & FILLET & & & \\
\hline WS-3 & 8/27/01 & BROOK & $\mathrm{F}$ & 114 & 17 & FILLET & & & \\
\hline WS-3 & $8 / 27 / 01$ & BROOK & $F$ & 142 & 28 & FILLET & & & \\
\hline WS-3 & $8 / 27 / 01$ & BROOK & M & 115 & 14 & FILLET & & & \\
\hline WS-3 & $8 / 27 / 01$ & BROOK & - & 97 & 8 & FILLET & MT-WS3-FIL2 & 17.28 & 15.82 \\
\hline WS-3 & $8 / 27 / 01$ & BROOK & M & 110 & 14 & FILLET & & & \\
\hline WS-3 & $8 / 27 / 01$ & BROOK & $M$ & 112 & 13 & FILLET & & & \\
\hline WS-3 & $8 / 27 / 01$ & BROOK & - & 105 & 9 & FILLET & & & \\
\hline WS-3 & $8 / 27 / 01$ & BROOK & $M$ & 120 & 19 & FILLET & & & \\
\hline WS-3 & 8/27/01 & BROOK & M & 132 & 26 & FILLET & & & \\
\hline WS-3 & 8/27/01 & BROOK & $\mathrm{F}$ & 100 & 9 & FILLET & MT-WS3-FIL3 & 16.89 & 14.75 \\
\hline WS-3 & $8 / 27 / 01$ & BROOK & - & 105 & 9 & FILLET & & & \\
\hline WS-3 & $8 / 27 / 01$ & BROOK & M & 102 & 10 & FILLET & & & \\
\hline WS-3 & 8/27/01 & BROOK & - & 108 & 12 & FILLET & & & \\
\hline WS-3 & $8 / 27 / 01$ & BROOK & - & 111 & 13 & FILLET & & & \\
\hline WS-3 & $8 / 27 / 01$ & BROOK & M & 125 & 20 & FILLET & & & \\
\hline WS-3 & $8 / 27 / 01$ & BROOK & M & 112 & 12 & FILLET & MT-WS3- FIL4 & 17.24 & 15.83 \\
\hline WS-3 & $8 / 27 / 01$ & BROOK & - & 109 & 10 & FILLET & & & \\
\hline WS-3 & 8/27/01 & BROOK & M & 110 & 13 & FILLET & & & \\
\hline WS-3 & 8/27/01 & BROOK & - & 105 & 9 & FILLET & & & \\
\hline WS-3 & 8/27/01 & BROOK & - & 98 & 9 & FILLET & & & \\
\hline
\end{tabular}


Metallothionein

\begin{tabular}{|c|c|c|c|c|c|c|c|c|}
\hline Site ID & Date & Species & Sex & $\begin{array}{l}\text { Length } \\
(\mathrm{mm})\end{array}$ & $\begin{array}{c}\text { Weight } \\
(\mathrm{gm})\end{array}$ & Tissue & Sample ID & $\begin{array}{c}\text { Metallothionein } \\
(\mu \mathrm{g} / \mathrm{g})\end{array}$ \\
\hline PP-1 & $8 / 29 / 01$ & BROOK & $\mathrm{F}$ & 185 & 82 & LIVER & MT-PP1-LIV1 & 135.6 \\
\hline PP-1 & $8 / 29 / 01$ & BROOK & M & 222 & 130 & LIVER & & \\
\hline PP-1 & 8/29/01 & BROOK & $\mathrm{F}$ & 200 & 82 & LIVER & MT-PP1-LIV2 & 118.5 \\
\hline PP-1 & $8 / 29 / 01$ & BROOK & M & 180 & 63 & LIVER & & \\
\hline PP-1 & 8/29/01 & BROOK & $\mathrm{F}$ & 190 & 70 & LIVER & MT-PP1-LIV3 & 130.6 \\
\hline PP-1 & $8 / 29 / 01$ & BROOK & M & 188 & 86 & LIVER & & \\
\hline PP-1 & $8 / 29 / 01$ & BROOK & M & 168 & 60 & LIVER & & \\
\hline PP-1 & $8 / 29 / 01$ & BROOK & $\mathrm{F}$ & 206 & 93 & LIVER & MT-PP1-LIV4 & 51.5 \\
\hline PP-1 & $8 / 29 / 01$ & BROOK & M & 174 & 51 & LIVER & & \\
\hline PP-1 & $8 / 29 / 01$ & BROOK & M & 194 & 84 & LIVER & MT-PP1-LIV5 & 103.9 \\
\hline PP-1 & $8 / 29 / 01$ & BROOK & $\mathrm{F}$ & 199 & 77 & LIVER & & \\
\hline PP-1 & $8 / 29 / 01$ & BROOK & $\mathrm{F}$ & 176 & 60 & LIVER & MT-PP1-LIV6 & 54.0 \\
\hline PP-1 & $8 / 29 / 01$ & BROOK & - & 205 & 95 & LIVER & & \\
\hline PP-1 & $8 / 29 / 01$ & BROOK & $\mathrm{M}$ & 200 & 103 & LIVER & MT-PP1-LIV7 & 93.7 \\
\hline PP-1 & $8 / 29 / 01$ & BROOK & $\mathrm{M}$ & 210 & 121 & LIVER & & \\
\hline PP-1 & $8 / 29 / 01$ & BROOK & $\mathrm{F}$ & 185 & 82 & GILL & MT-PP1-GIL1 & 16.1 \\
\hline PP-1 & $8 / 29 / 01$ & BROOK & $\mathrm{M}$ & 222 & 130 & GILL & & \\
\hline PP-1 & $8 / 29 / 01$ & BROOK & $\mathrm{F}$ & 200 & 82 & GILL & MT-PP1-GIL2 & 20.9 \\
\hline PP-1 & 8/29/01 & BROOK & M & 180 & 63 & GILL & & \\
\hline PP-1 & $8 / 29 / 01$ & BROOK & $\mathrm{F}$ & 190 & 70 & GILL & & \\
\hline PP-1 & 8/29/01 & BROOK & M & 188 & 86 & GILL & MT-PP1-GIL3 & 38.1 \\
\hline PP-1 & $8 / 29 / 01$ & BROOK & $\mathrm{M}$ & 168 & 60 & GILL & & \\
\hline PP-1 & $8 / 29 / 01$ & BROOK & $\mathrm{F}$ & 206 & 93 & GILL & MT-PP1-GIL4 & 44.0 \\
\hline PP-1 & $8 / 29 / 01$ & BROOK & $\mathrm{M}$ & 174 & 51 & GILL & & \\
\hline PP-1 & 8/29/01 & BROOK & $\mathrm{M}$ & 194 & 84 & GILL & MT-PP1-GIL5 & 75.9 \\
\hline PP-1 & $8 / 29 / 01$ & BROOK & $\mathrm{F}$ & 199 & 77 & GILL & & \\
\hline PP-1 & $8 / 29 / 01$ & BROOK & $\mathrm{F}$ & 176 & 60 & GILL & MT-PP1-GIL6 & 48.1 \\
\hline PP-1 & $8 / 29 / 01$ & BROOK & - & 205 & 95 & GILL & & \\
\hline PP-1 & $8 / 29 / 01$ & BROOK & $\mathrm{M}$ & 200 & 103 & GILL & MT-PP1-GIL7 & 101.2 \\
\hline PP-1 & $8 / 29 / 01$ & BROOK & M & 210 & 121 & GILL & & \\
\hline PP-1 & 8/29/01 & BROOK & $\bar{F}$ & 185 & 82 & CAECA & MT-PP1-PYC1 & 316.2 \\
\hline PP-1 & $8 / 29 / 01$ & BROOK & M & 222 & 130 & CAECA & & \\
\hline PP-1 & 8/29/01 & BROOK & $\mathrm{F}$ & 200 & 82 & CAECA & MT-PP1-PYC2 & 301.0 \\
\hline PP-1 & $8 / 29 / 01$ & BROOK & $\mathrm{M}$ & 180 & 63 & CAECA & & \\
\hline PP-1 & 8/29/01 & BROOK & $\mathrm{F}$ & 190 & 70 & CAECA & MT-PP1-PYC3 & 367.8 \\
\hline PP-1 & $8 / 29 / 01$ & BROOK & $\mathrm{M}$ & 188 & 86 & CAECA & & \\
\hline PP-1 & 8/29/01 & BROOK & $\mathrm{M}$ & 168 & 60 & CAECA & & \\
\hline PP-1 & $8 / 29 / 01$ & BROOK & $\mathrm{F}$ & 206 & 93 & CAECA & MT-PP1-PYC4 & 322.4 \\
\hline PP-1 & $8 / 29 / 01$ & BROOK & M & 174 & 51 & CAECA & & \\
\hline PP-1 & $8 / 29 / 01$ & BROOK & $\mathrm{M}$ & 194 & 84 & CAECA & MT-PP1-PYC5 & 510.0 \\
\hline PP-1 & $8 / 29 / 01$ & BROOK & $\mathrm{F}$ & 199 & 77 & CAECA & & \\
\hline PP-1 & $8 / 29 / 01$ & BROOK & $\mathrm{F}$ & 176 & 60 & CAECA & MT-PP1-PYC6 & 332.5 \\
\hline PP-1 & $8 / 29 / 01$ & BROOK & - & 205 & 95 & CAECA & & \\
\hline PP-1 & 8/29/01 & BROOK & M & 200 & 103 & CAECA & MT-PP1-PYC7 & 161.7 \\
\hline PP-1 & $8 / 29 / 01$ & BROOK & M & 210 & 121 & CAECA & & \\
\hline PP-1 & 8/29/01 & BROOK & $\bar{F}$ & 185 & 82 & \begin{tabular}{c|} 
FILLET \\
\end{tabular} & "MT-PP1-FIL1 & 26.5 \\
\hline PP-1 & $8 / 29 / 01$ & BROOK & M & 222 & 130 & FILLET & & \\
\hline PP-1 & $8 / 29 / 01$ & BROOK & $\mathrm{F}$ & 200 & 82 & FILLET & MT-PP1-FIL2 & 6.2 \\
\hline PP-1 & $8 / 29 / 01$ & BROOK & M & 180 & 63 & FILLET & & \\
\hline PP-1 & 8/29/01 & BROOK & $\mathrm{F}$ & 190 & 70 & FILLET & MT-PP1-FIL3 & 10.3 \\
\hline PP-1 & $8 / 29 / 01$ & BROOK & M & 188 & 86 & FILLET & & \\
\hline PP-1 & $8 / 29 / 01$ & BROOK & M & 168 & 60 & FILLET & & \\
\hline PP-1 & 8/29/01 & BROOK & $\mathrm{F}$ & 206 & 93 & FILLET & MT-PP1-FIL4 & 7.2 \\
\hline PP-1 & $8 / 29 / 01$ & BROOK & M & 174 & 51 & FILLET & & \\
\hline
\end{tabular}


Metallothionein (Continued)

\begin{tabular}{|c|c|c|c|c|c|c|c|c|}
\hline Site ID & Date & Species & Sex & Length & Weight & Tissue & Sample ID & $\begin{array}{c}\text { Metallothionein } \\
(\mu \mathrm{g} / \mathrm{g})\end{array}$ \\
\hline PP-1 & $8 / 29 / 01$ & BROOK & $M$ & 194 & 84 & FILLET & "MT-PP1-FIL5 & 4.90 \\
\hline PP-1 & $8 / 29 / 01$ & BROOK & $\mathrm{F}$ & 199 & 77 & FILLET & & \\
\hline PP-1 & $8 / 29 / 01$ & BROOK & $F$ & 176 & 60 & FILLET & MT-PP1-FIL6 & 17.1 \\
\hline PP-1 & $8 / 29 / 01$ & BROOK & - & 205 & 95 & FILLET & & \\
\hline PP-1 & $8 / 29 / 01$ & BROOK & $\mathrm{M}$ & 200 & 103 & FILLET & MT-PP1-FIL7 & 39.0 \\
\hline PP-1 & $8 / 29 / 01$ & BROOK & M & 210 & 121 & FILLET & & \\
\hline PP-3 & $8 / 28 / 01$ & BROOK & $\mathrm{F}$ & 164 & 43 & LIVER & & \\
\hline PP-3 & $8 / 28 / 01$ & BROOK & M & 167 & 39 & LIVER & MT-PP3-LIV1 & 429.6 \\
\hline PP-3 & $8 / 28 / 01$ & BROOK & $M$ & 134 & 20 & LIVER & & \\
\hline PP-3 & $8 / 28 / 01$ & BROOK & $M$ & 167 & 43 & LIVER & & \\
\hline PP-3 & $8 / 28 / 01$ & BROOK & $M$ & 151 & 32 & LIVER & MT-PP3-LIV2 & 553.5 \\
\hline PP-3 & $8 / 28 / 01$ & BROOK & $\mathrm{F}$ & 139 & 28 & LIVER & & \\
\hline PP-3 & $8 / 28 / 01$ & BROOK & $\mathrm{F}$ & 140 & 22 & LIVER & & \\
\hline PP-3 & $8 / 28 / 01$ & BROOK & M & 163 & 39 & LIVER & MT-PP3-LIV3 & 1,057 \\
\hline PP-3 & $8 / 28 / 01$ & BROOK & M & 169 & 46 & LIVER & & \\
\hline PP-3 & $8 / 28 / 01$ & BROOK & $M$ & 190 & 76 & LIVER & MT-PP3-LIV4 & 480.2 \\
\hline PP-3 & $8 / 28 / 01$ & BROOK & $M$ & 150 & 32 & LIVER & & \\
\hline PP-3 & $8 / 28 / 01$ & BROOK & $\mathrm{F}$ & 153 & 43 & LIVER & & \\
\hline PP-3 & 8/28/01 & BROOK & $\mathrm{F}$ & 147 & 28 & LIVER & MT-PP3-LIV5 & 523.9 \\
\hline PP-3 & $8 / 28 / 01$ & BROOK & $M$ & 151 & 29 & LIVER & & \\
\hline PP-3 & $8 / 28 / 01$ & BROOK & $\mathrm{F}$ & 243 & 140 & LIVER & MT-PP3-LIV6 & 407.4 \\
\hline PP-3 & $8 / 28 / 01$ & RAINBOW & $F$ & 247 & 140 & LIVER & MT-PP3-LIV7 & 574.3 \\
\hline PP-3 & $8 / 28 / 01$ & RAINBOW & $M$ & 268 & 194 & LIVER & MT-PP3-LIV8 & 464.4 \\
\hline PP-3 & $8 / 28 / 01$ & RAINBOW & $M$ & 265 & 176 & LIVER & MT-PP3-LIV9 & 275.2 \\
\hline PP-3 & $8 / 28 / 01$ & RAINBOW & $\mathrm{F}$ & 302 & 222 & LIVER & MT-PP3-LIV10 & 261.6 \\
\hline PP-3 & $8 / 28 / 01$ & RAINBOW & $\mathrm{F}$ & 302 & 258 & LIVER & MT-PP3-LIV11 & 121.1 \\
\hline PP-3 & 8/28/01 & BROOK & $F$ & 164 & 43 & GILL & & \\
\hline PP3 & $8 / 28 / 01$ & BROOK & M & 167 & 39 & GILL & MT-PP3-GIL1 & 196.9 \\
\hline PP-3 & $8 / 28 / 01$ & BROOK & $\mathrm{M}$ & 134 & 20 & GILL & & \\
\hline PP-3 & $8 / 28 / 01$ & BROOK & $M$ & 167 & 43 & GILL & & \\
\hline PP-3 & $8 / 28 / 01$ & BROOK & $M$ & 151 & 32 & GILL & MT-PP3-GIL2 & 264.6 \\
\hline PP-3 & $8 / 28 / 01$ & BROOK & $\mathrm{F}$ & 139 & 28 & GILL & & \\
\hline PP-3 & $8 / 28 / 01$ & BROOK & $F$ & 140 & 22 & GILL & & \\
\hline PP-3 & $8 / 28 / 01$ & BROOK & M & 163 & 39 & GILL & MT-PP3-GIL3 & 123.2 \\
\hline PP-3 & $8 / 28 / 01$ & BROOK & M & 169 & 46 & GILL & & \\
\hline PP-3 & $8 / 28 / 01$ & BROOK & $\mathrm{M}$ & 190 & 76 & GILL & MT-PP3-GIL4 & 293.8 \\
\hline PP-3 & $8 / 28 / 01$ & BROOK & M & 150 & 32 & GILL & & \\
\hline PP-3 & $8 / 28 / 01$ & BROOK & $\mathrm{F}$ & 153 & 43 & GILL & & \\
\hline PP-3 & $8 / 28 / 01$ & BROOK & $\mathrm{F}$ & 147 & 28 & GILL & MT-PP3-GIL5 & 143.4 \\
\hline PP-3 & $8 / 28 / 01$ & BROOK & $\mathrm{M}$ & 151 & 29 & GILL & & \\
\hline PP-3 & $8 / 28 / 01$ & BROOK & $\mathrm{F}$ & 243 & 140 & GILL & MT-PP3-GIL6 & 182.0 \\
\hline PP-3 & $8 / 28 / 01$ & RAINBOW & $\mathrm{F}$ & 247 & 140 & GILL & MT-PP3-GIL7 & 60.9 \\
\hline PP-3 & $8 / 28 / 01$ & RAINBOW & $M$ & 268 & 194 & GILL & MT-PP3-GIL8 & 69.9 \\
\hline PP-3 & $8 / 28 / 01$ & RAINBOW & $M$ & 265 & 176 & GILL & MT-PP3-GIL9 & 109.7 \\
\hline PP-3 & $8 / 28 / 01$ & RAINBOW & $\mathrm{F}$ & 302 & 222 & GILL & MT-PP3-GIL10 & 96.4 \\
\hline PP-3 & $8 / 28 / 01$ & RAINBOW & $\mathrm{F}$ & 302 & 258 & GILL & MT-PP3-GIL11 & 87.4 \\
\hline PP-3 & $8 / 28 / 01$ & BROOK & $F$ & 164 & 43 & CAECA & & \\
\hline PP-3 & $8 / 28 / 01$ & BROOK & M & 167 & 39 & CAECA & MT-PP3-PYC1 & 699.2 \\
\hline PP-3 & $8 / 28 / 01$ & BROOK & $\mathrm{M}$ & 134 & 20 & CAECA & & \\
\hline PP-3 & $8 / 28 / 01$ & BROOK & $M$ & 167 & 43 & CAECA & MT-PP3-PYC2 & 665.9 \\
\hline
\end{tabular}


Metallothionein (Continued)

\begin{tabular}{|c|c|c|c|c|c|c|c|c|}
\hline Site ID & Date & Species & Sex & Length & Weight & Tissue & Sample ID & $\begin{array}{c}\text { Metallothionein } \\
(\mu \mathrm{g} / \mathrm{g})\end{array}$ \\
\hline PP-3 & $8 / 28 / 01$ & BROOK & $\mathrm{M}$ & 151 & 32 & CAECA & & \\
\hline PP-3 & $8 / 28 / 01$ & BROOK & $M$ & 163 & 39 & CAECA & MT-PP3-PYC3 & 621.8 \\
\hline PP-3 & $8 / 28 / 01$ & BROOK & M & 169 & 46 & CAECA & & \\
\hline PP-3 & 8/28/01 & BROOK & M & 190 & 76 & CAECA & MT-PP3-PYC4 & 898.2 \\
\hline PP-3 & $8 / 28 / 01$ & BROOK & M & 150 & 32 & CAECA & & \\
\hline PP-3 & 8/28/01 & BROOK & $\mathrm{F}$ & 153 & 43 & CAECA & & \\
\hline PP-3 & $8 / 28 / 01$ & BROOK & $\mathrm{F}$ & 147 & 28 & CAECA & MT-PP3-PYC5 & 318.5 \\
\hline PP-3 & $8 / 28 / 01$ & BROOK & $M$ & 151 & 29 & CAECA & & \\
\hline PP-3 & $8 / 28 / 01$ & BROOK & $\mathrm{F}$ & 243 & 140 & CAECA & MT-PP3-PYC6 & 708.0 \\
\hline PP-3 & $8 / 28 / 01$ & RAINBOW & $\mathrm{F}$ & 247 & 140 & CAECA & MT-PP3-PYC7 & 771.7 \\
\hline PP-3 & $8 / 28 / 01$ & RAINBOW & $M$ & 268 & 194 & CAECA & MT-PP3-PYC8 & 646.4 \\
\hline PP-3 & $8 / 28 / 01$ & RAINBOW & $M$ & 265 & 176 & CAECA & MT-PP3-PYC9 & 838.8 \\
\hline PP-3 & 8/28/01 & RAINBOW & $\mathrm{F}$ & 302 & 222 & CAECA & MT-PP3-PYC10 & 496.9 \\
\hline PP-3 & $8 / 28 / 01$ & RAINBOW & $\mathrm{F}$ & 302 & 258 & CAECA & MT-PP3-PYC11 & 1,178 \\
\hline PP-3 & $8 / 28 / 01$ & BROOK & $\mathrm{F}$ & 164 & 43 & FILLET & & \\
\hline PP-3 & $8 / 28 / 01$ & BROOK & M & 167 & 39 & FILLET & MT-PP3-FIL1 & 25.7 \\
\hline PP-3 & $8 / 28 / 01$ & BROOK & $M$ & 134 & 20 & FILLET & & \\
\hline PP-3 & $8 / 28 / 01$ & BROOK & $M$ & 167 & 43 & FILLET & & \\
\hline PP-3 & $8 / 28 / 01$ & BROOK & $M$ & 151 & 32 & FILLET & MT-PP3-FIL2 & 17.6 \\
\hline PP-3 & $8 / 28 / 01$ & BROOK & $\mathrm{F}$ & 139 & 28 & FILLET & & \\
\hline PP-3 & $8 / 28 / 01$ & BROOK & $F$ & 140 & 22 & FILLET & & \\
\hline PP-3 & $8 / 28 / 01$ & BROOK & M & 163 & 39 & FILLET & MT-PP3-FIL3 & - \\
\hline PP-3 & $8 / 28 / 01$ & BROOK & $M$ & 169 & 46 & FILLET & & \\
\hline PP-3 & $8 / 28 / 01$ & BROOK & M & 190 & 76 & FILLET & MT-PP3-FIL4 & - \\
\hline PP-3 & $8 / 28 / 01$ & BROOK & $M$ & 150 & 32 & FILLET & & \\
\hline PP-3 & $8 / 28 / 01$ & BROOK & $F$ & 153 & 43 & FILLET & & \\
\hline PP-3 & $8 / 28 / 01$ & BROOK & $\mathrm{F}$ & 147 & 28 & FILLET & MT-PP3-FIL5 & 509.7 \\
\hline PP-3 & $8 / 28 / 01$ & BROOK & M & 151 & 29 & FILLET & & \\
\hline PP-3 & $8 / 28 / 01$ & BROOK & $\mathrm{F}$ & 243 & 140 & FILLET & MT-PP3-FIL6 & 1,611 \\
\hline PP-3 & $8 / 28 / 01$ & RAINBOW & $\mathrm{F}$ & 247 & 140 & FILLET & MT-PP3-FIL7 & 285.0 \\
\hline PP-3 & $8 / 28 / 01$ & RAINBOW & $\mathrm{M}$ & 268 & 194 & FILLET & MT-PP3-FIL8 & 78.0 \\
\hline PP-3 & $8 / 28 / 01$ & RAINBOW & $\mathrm{M}$ & 265 & 176 & FILLET & MT-PP3-FIL9 & - \\
\hline PP-3 & $8 / 28 / 01$ & RAINBOW & $\mathrm{F}$ & 302 & 222 & FILLET & MT-PP3-FIL10 & 155.7 \\
\hline PP-3 & $8 / 28 / 01$ & RAINBOW & $\mathrm{F}$ & 302 & 258 & FILLET & MT-PP3-FIL11 & 104.5 \\
\hline PP-5 & $8 / 28 / 01$ & "RAINBOW & $\bar{M}$ & 153 & 37 & "LIVER & & \\
\hline PP-5 & $8 / 28 / 01$ & RAINBOW & M & 167 & 42 & LIVER & MT-PP5-LIV1 & 114.0 \\
\hline PP-5 & 8/28/01 & RAINBOW & M & 154 & 36 & LIVER & & \\
\hline PP-5 & $8 / 28 / 01$ & RAINBOW & $F$ & 155 & 34 & LIVER & MT-PP5-LIV2 & 50.4 \\
\hline PP-5 & 8/28/01 & RAINBOW & $M$ & 220 & 106 & LIVER & & \\
\hline PP-5 & $8 / 28 / 01$ & RAINBOW & $M$ & 222 & 99 & LIVER & MT-PP5-LIV3 & 43.1 \\
\hline PP-5 & $8 / 28 / 01$ & RAINBOW & $\mathrm{F}$ & 161 & 43 & LIVER & & \\
\hline PP-5 & $8 / 28 / 01$ & RAINBOW & $M$ & 195 & 76 & LIVER & MT-PP5-LIV4 & 263.9 \\
\hline PP-5 & $8 / 28 / 01$ & RAINBOW & $\mathrm{F}$ & 167 & 46 & LIVER & & \\
\hline PP-5 & $8 / 28 / 01$ & RAINBOW & $M$ & 185 & 64 & LIVER & MT-PP5-LIV5 & 294.1 \\
\hline PP-5 & $8 / 28 / 01$ & RAINBOW & $\mathrm{F}$ & 174 & 49 & LIVER & & \\
\hline PP-5 & $8 / 28 / 01$ & RAINBOW & $M$ & 173 & 50 & LIVER & MT-PP5-LIV6 & 182.9 \\
\hline PP-5 & $8 / 28 / 01$ & RAINBOW & M & 154 & 60 & LIVER & & \\
\hline PP-5 & $8 / 28 / 01$ & RAINBOW & $M$ & 182 & 60 & LIVER & MT-PP5-LIV7 & 54.1 \\
\hline PP-5 & $8 / 28 / 01$ & RAINBOW & $\mathrm{F}$ & 168 & 46 & LIVER & & \\
\hline PP-5 & $8 / 28 / 01$ & RAINBOW & $M$ & 153 & 37 & GILL & & \\
\hline PP-5 & $8 / 28 / 01$ & RAINBOW & $M$ & 167 & 42 & GILL & MT-PP5-GIL1 & 186.9 \\
\hline PP-5 & $8 / 28 / 01$ & RAINBOW & M & 154 & 36 & GILL & & \\
\hline PP-5 & $8 / 28 / 01$ & RAINBOW & $\mathrm{F}$ & 155 & 34 & GILL & MT-PP5-GIL2 & 167.9 \\
\hline PP-5 & 8/28/01 & RAINBOW & M & 220 & 106 & GILL & & \\
\hline
\end{tabular}


Metallothionein (Continued)

\begin{tabular}{|c|c|c|c|c|c|c|c|c|}
\hline Site ID & Date & Species & Sex & Length & Weight & Tissue & Sample ID & $\begin{array}{c}\text { Metallothionein } \\
(\mu \mathrm{g} / \mathrm{g})\end{array}$ \\
\hline PP-5 & $8 / 28 / 01$ & RAINBOW & $M$ & 222 & 99 & GILL & MT-PP5-GIL3 & 111.3 \\
\hline PP-5 & $8 / 28 / 01$ & RAINBOW & $\mathrm{F}$ & 161 & 43 & GILL & & \\
\hline PP-5 & $8 / 28 / 01$ & RAINBOW & $M$ & 195 & 76 & GILL & MT-PP5-GIL4 & 126.7 \\
\hline PP-5 & $8 / 28 / 01$ & RAINBOW & $\mathrm{F}$ & 167 & 46 & GILL & & \\
\hline PP-5 & $8 / 28 / 01$ & RAINBOW & $M$ & 185 & 64 & GILL & MT-PP5-GIL5 & 53.2 \\
\hline PP-5 & $8 / 28 / 01$ & RAINBOW & $\mathrm{F}$ & 174 & 49 & GILL & & \\
\hline PP-5 & $8 / 28 / 01$ & RAINBOW & $\mathrm{M}$ & 173 & 50 & GILL & MT-PP5-GIL6 & 126.1 \\
\hline PP-5 & $8 / 28 / 01$ & RAINBOW & M & 154 & 60 & GILL & & \\
\hline PP-5 & $8 / 28 / 01$ & RAINBOW & $M$ & 182 & 60 & GILL & MT-PP5-LIV7 & 35.4 \\
\hline PP-5 & $8 / 28 / 01$ & RAINBOW & $\mathrm{F}$ & 168 & 46 & GILL & & \\
\hline PP-5 & $8 / 28 / 01$ & RAINBOW & M & 153 & 37 & CAECA & & \\
\hline PP-5 & $8 / 28 / 01$ & RAINBOW & M & 167 & 42 & CAECA & MT-PP5-PYC1 & 634.3 \\
\hline PP-5 & $8 / 28 / 01$ & RAINBOW & M & 154 & 36 & CAECA & & \\
\hline PP-5 & $8 / 28 / 01$ & RAINBOW & $\mathrm{F}$ & 155 & 34 & CAECA & MT-PP5-PYC2 & - \\
\hline PP-5 & $8 / 28 / 01$ & RAINBOW & $\mathrm{M}$ & 220 & 106 & CAECA & & \\
\hline PP-5 & $8 / 28 / 01$ & RAINBOW & $M$ & 222 & 99 & CAECA & MT-PP5-PYC3 & 544.3 \\
\hline PP-5 & $8 / 28 / 01$ & RAINBOW & $\mathrm{F}$ & 161 & 43 & CAECA & & \\
\hline PP-5 & $8 / 28 / 01$ & RAINBOW & $M$ & 195 & 76 & CAECA & MT-PP5-PYC4 & - \\
\hline PP-5 & $8 / 28 / 01$ & RAINBOW & $\mathrm{F}$ & 167 & 46 & CAECA & & \\
\hline PP-5 & $8 / 28 / 01$ & RAINBOW & $M$ & 185 & 64 & CAECA & MT-PP5-PYC5 & 513.8 \\
\hline PP-5 & $8 / 28 / 01$ & RAINBOW & $\mathrm{F}$ & 174 & 49 & CAECA & & \\
\hline PP-5 & $8 / 28 / 01$ & RAINBOW & $M$ & 173 & 50 & CAECA & MT-PP5-PYC6 & - \\
\hline PP-5 & $8 / 28 / 01$ & RAINBOW & $\mathrm{M}$ & 154 & 60 & CAECA & & \\
\hline PP-5 & $8 / 28 / 01$ & RAINBOW & $M$ & 182 & 60 & CAECA & MT-PP5-PYC7 & - \\
\hline PP-5 & $8 / 28 / 01$ & RAINBOW & $\mathrm{F}$ & 168 & 46 & CAECA & & \\
\hline PP-5 & $8 / 28 / 01$ & RAINBOW & M & 153 & 37 & FILLET & & \\
\hline PP-5 & $8 / 28 / 01$ & RAINBOW & M & 167 & 42 & FILLET & MT-PP5-FIL1 & 148.4 \\
\hline PP-5 & $8 / 28 / 01$ & RAINBOW & $\mathrm{M}$ & 154 & 36 & FILLET & & \\
\hline PP-5 & $8 / 28 / 01$ & RAINBOW & $\mathrm{F}$ & 155 & 34 & FILLET & MT-PP5-FIL2 & 51.3 \\
\hline PP-5 & $8 / 28 / 01$ & RAINBOW & $\mathrm{M}$ & 220 & 106 & FILLET & & \\
\hline PP-5 & $8 / 28 / 01$ & RAINBOW & $M$ & 222 & 99 & FILLET & MT-PP5-FIL3 & 32.9 \\
\hline PP-5 & $8 / 28 / 01$ & RAINBOW & $\mathrm{F}$ & 161 & 43 & FILLET & & \\
\hline PP-5 & $8 / 28 / 01$ & RAINBOW & $M$ & 195 & 76 & FILLET & MT-PP5-FIL4 & 83.8 \\
\hline PP-5 & 8/28/01 & RAINBOW & $\mathrm{F}$ & 167 & 46 & FILLET & & \\
\hline PP-5 & $8 / 28 / 01$ & RAINBOW & $\mathrm{M}$ & 185 & 64 & FILLET & MT-PP5-FIL5 & 38.3 \\
\hline PP-5 & $8 / 28 / 01$ & RAINBOW & $\mathrm{F}$ & 174 & 49 & FILLET & & \\
\hline PP-5 & $8 / 28 / 01$ & RAINBOW & $M$ & 173 & 50 & FILLET & MT-PP5-FIL6 & 59.0 \\
\hline PP-5 & 8/28/01 & RAINBOW & $\mathrm{M}$ & 154 & 60 & FILLET & & \\
\hline PP-5 & $8 / 28 / 01$ & RAINBOW & $\mathrm{M}$ & 182 & 60 & FILLET & MT-PP5-FIL7 & 33.3 \\
\hline PP-5 & $8 / 28 / 01$ & RAINBOW & $\mathrm{F}$ & 168 & 46 & FILLET & & \\
\hline ULGP & $8 / 29 / 01$ & WSC & $\mathrm{M}$ & 420 & 747 & LIVER & MT-ULG-LIV1 & 81.5 \\
\hline ULGP & $8 / 29 / 01$ & WSC & $\mathrm{M}$ & 302 & 248 & LIVER & MT-ULG-LIV2 & 1043 \\
\hline ULGP & $8 / 29 / 01$ & WSC & $\mathrm{M}$ & 312 & 270 & LIVER & MT-ULG-LIV3 & 97.7 \\
\hline ULGP & $8 / 29 / 01$ & WSC & $\mathrm{M}$ & 294 & 253 & LIVER & MT-ULG-LIV4 & 36.1 \\
\hline ULGP & $8 / 29 / 01$ & WSC & $\mathrm{M}$ & 263 & 174 & LIVER & MT-ULG-LIV5 & 90.5 \\
\hline ULGP & $8 / 29 / 01$ & WSC & $\mathrm{M}$ & 260 & 177 & LIVER & MT-ULG-LIV6 & 141.4 \\
\hline ULGP & $8 / 29 / 01$ & WSC & $\mathrm{M}$ & 420 & 747 & GILL & MT-ULG-LIV1 & 46.7 \\
\hline ULGP & $8 / 29 / 01$ & WSC & $\mathrm{M}$ & 302 & 248 & GILL & MT-ULG-LIV2 & 46.7 \\
\hline ULGP & $8 / 29 / 01$ & WSC & $\mathrm{M}$ & 312 & 270 & GILL & MT-ULG-LIV3 & 33.5 \\
\hline ULGP & $8 / 29 / 01$ & WSC & $\mathrm{M}$ & 294 & 253 & GILL & MT-ULG-LIV4 & 41.7 \\
\hline ULGP & $8 / 29 / 01$ & WSC & $M$ & 263 & 174 & GILL & MT-ULG-LIV5 & 94.5 \\
\hline ULGP & $8 / 29 / 01$ & WSC & $\mathrm{M}$ & 260 & 177 & GILL & MT-ULG-LIV6 & 46.9 \\
\hline ULGP & $8 / 29 / 01$ & WSC & $\mathrm{M}$ & 420 & 747 & CAECA & MT-ULG-PYC1 & 279.9 \\
\hline ULGP & $8 / 29 / 01$ & WSC & $\mathrm{M}$ & 302 & 248 & CAECA & MT-ULG-PYC2 & 342.0 \\
\hline
\end{tabular}


Metallothionein (Continued)

\begin{tabular}{|c|c|c|c|c|c|c|c|c|}
\hline Site ID & Date & Species & Sex & Length & Weight & Tissue & Sample ID & $\begin{array}{c}\text { Metallothionein } \\
(\mu g / g)\end{array}$ \\
\hline ULGP & $8 / 29 / 01$ & WSC & $M$ & 312 & 270 & CAECA & MT-ULG-PYC3 & 275.8 \\
\hline ULGP & $8 / 29 / 01$ & WSC & $M$ & 294 & 253 & CAECA & MT-ULG-PYC4 & 315.5 \\
\hline ULGP & $8 / 29 / 01$ & WSC & $M$ & 263 & 174 & CAECA & MT-ULG-PYC5 & 415.7 \\
\hline ULGP & $8 / 29 / 01$ & WSC & $\mathrm{M}$ & 260 & 177 & CAECA & MT-ULG-PYC6 & 484.7 \\
\hline ULGP & $8 / 29 / 01$ & WSC & $\mathrm{M}$ & 302 & 248 & FILLET & MT-ULG-FIL2 & 2.0 \\
\hline ULGP & $8 / 29 / 01$ & WSC & $M$ & 312 & 270 & FILLET & MT-ULG-FIL3 & 49.2 \\
\hline ULGP & $8 / 29 / 01$ & WSC & $M$ & 294 & 253 & FILLET & MT-ULG-FIL4 & 29.9 \\
\hline ULGP & $8 / 29 / 01$ & WSC & $M$ & 263 & 174 & FILLET & MT-ULG-FIL5 & 39.4 \\
\hline ULGP & $8 / 29 / 01$ & WSC & $\mathrm{M}$ & 260 & 177 & FILLET & MT-ULG-FIL6 & 49.7 \\
\hline WS-3 & $8 / 27 / 01$ & BROOK & $\mathrm{M}$ & 127 & 23 & LIVER & & \\
\hline WS-3 & 8/27/01 & BROOK & $\mathrm{F}$ & 111 & 12 & LIVER & MT-WS3-LIV1 & 273.4 \\
\hline WS-3 & $8 / 27 / 01$ & BROOK & $\mathrm{F}$ & 114 & 15 & LIVER & & \\
\hline WS-3 & $8 / 27 / 01$ & BROOK & - & 100 & 9 & LIVER & & \\
\hline WS-3 & $8 / 27 / 01$ & BROOK & $\mathrm{F}$ & 114 & 17 & LIVER & & \\
\hline WS-3 & $8 / 27 / 01$ & BROOK & $\mathrm{F}$ & 142 & 28 & LIVER & & \\
\hline WS-3 & $8 / 27 / 01$ & BROOK & $M$ & 115 & 14 & LIVER & & \\
\hline WS-3 & 8/27/01 & BROOK & - & 97 & 8 & LIVER & MT-WS3-LIV2 & 36.1 \\
\hline WS-3 & $8 / 27 / 01$ & BROOK & $M$ & 110 & 14 & LIVER & & \\
\hline WS-3 & 8/27/01 & BROOK & $M$ & 112 & 13 & LIVER & & \\
\hline WS-3 & $8 / 27 / 01$ & BROOK & - & 105 & 9 & LIVER & & \\
\hline WS-3 & $8 / 27 / 01$ & BROOK & $M$ & 120 & 19 & LIVER & & \\
\hline WS-3 & $8 / 27 / 01$ & BROOK & $M$ & 132 & 26 & LIVER & & \\
\hline WS-3 & $8 / 27 / 01$ & BROOK & $\mathrm{F}$ & 100 & 9 & LIVER & MT-WS3-LIV3 & 178.1 \\
\hline WS-3 & $8 / 27 / 01$ & BROOK & - & 108 & 12 & LIVER & & \\
\hline WS-3 & $8 / 27 / 01$ & BROOK & - & 111 & 13 & LIVER & & \\
\hline WS-3 & 8/27/01 & BROOK & $M$ & 125 & 20 & LIVER & & \\
\hline WS-3 & $8 / 27 / 01$ & BROOK & $M$ & 112 & 12 & LIVER & MT-WS3-LIV4 & 87.0 \\
\hline WS3 & 8/27/01 & BROOK & - & 109 & 10 & LIVER & & \\
\hline WS-3 & $8 / 27 / 01$ & BROOK & $M$ & 110 & 13 & LIVER & & \\
\hline WS-3 & $8 / 27 / 01$ & BROOK & - & 105 & 9 & LIVER & & \\
\hline WS-3 & $8 / 27 / 01$ & BROOK & - & 98 & 9 & LIVER & & \\
\hline WS-3 & $8 / 27 / 01$ & BROOK & $\bar{M}$ & 127 & 23 & "GILL & & \\
\hline WS-3 & 8/27/01 & BROOK & - & 93 & 10 & GILL & & \\
\hline WS-3 & $8 / 27 / 01$ & BROOK & $\mathrm{F}$ & 111 & 12 & GILL & MT-WS3-GIL1 & 211.6 \\
\hline WS-3 & 8/27/01 & BROOK & $\mathrm{F}$ & 114 & 15 & GILL & & \\
\hline WS-3 & $8 / 27 / 01$ & BROOK & - & 100 & 9 & GILL & & \\
\hline WS-3 & 8/27/01 & BROOK & $\mathrm{F}$ & 114 & 17 & GILL & & \\
\hline WS-3 & $8 / 27 / 01$ & BROOK & $\mathrm{F}$ & 142 & 28 & GILL & & \\
\hline WS-3 & $8 / 27 / 01$ & BROOK & $M$ & 115 & 14 & GILL & & \\
\hline WS-3 & 8/27/01 & BROOK & - & 97 & 8 & GILL & MT-WS3-GIL2 & 140.9 \\
\hline WS-3 & 8/27/01 & BROOK & $M$ & 110 & 14 & GILL & & \\
\hline WS-3 & $8 / 27 / 01$ & BROOK & $M$ & 112 & 13 & GILL & & \\
\hline WS-3 & $8 / 27 / 01$ & BROOK & - & 105 & 9 & GILL & & \\
\hline WS-3 & $8 / 27 / 01$ & BROOK & $M$ & 120 & 19 & GILL & & \\
\hline WS-3 & $8 / 27 / 01$ & BROOK & $M$ & 132 & 26 & GILL & & \\
\hline WS-3 & 8/27/01 & BROOK & $\mathrm{F}$ & 100 & 9 & GILL & MT-WS3-GIL3 & 159.9 \\
\hline WS-3 & $8 / 27 / 01$ & BROOK & - & 105 & 9 & GILL & & \\
\hline WS-3 & $8 / 27 / 01$ & BROOK & $M$ & 102 & 10 & GILL & & \\
\hline WS-3 & 8/27/01 & BROOK & - & 108 & 12 & GILL & & \\
\hline WS-3 & $8 / 27 / 01$ & BROOK & - & 111 & 13 & GILL & & \\
\hline
\end{tabular}


Metallothionein (Continued)

\begin{tabular}{|c|c|c|c|c|c|c|c|c|}
\hline Site ID & Date & Species & Sex & Length & Weight & Tissue & Sample ID & $\begin{array}{c}\text { Metallothionein } \\
(\mu \mathrm{g} / \mathrm{g})\end{array}$ \\
\hline WS-3 & $8 / 27 / 01$ & BROOK & $\mathrm{M}$ & 112 & 12 & GILL & MT-WS3-GIL4 & 300.9 \\
\hline WS-3 & $8 / 27 / 01$ & BROOK & - & 109 & 10 & GILL & & \\
\hline WS-3 & $8 / 27 / 01$ & BROOK & $M$ & 110 & 13 & GILL & & \\
\hline WS-3 & $8 / 27 / 01$ & BROOK & - & 105 & 9 & GILL & & \\
\hline WS-3 & $8 / 27 / 01$ & BROOK & - & 98 & 9 & GILL & & \\
\hline WS-3 & $8 / 27 / 01$ & BROOK & $\mathrm{M}$ & 127 & 23 & CAECA & & \\
\hline WS-3 & $8 / 27 / 01$ & BROOK & - & 93 & 10 & CAECA & & \\
\hline WS-3 & $8 / 27 / 01$ & BROOK & $\mathrm{F}$ & 111 & 12 & CAECA & MT-WS3-PYCL1 & 532.0 \\
\hline WS-3 & $8 / 27 / 01$ & BROOK & $\mathrm{F}$ & 114 & 15 & CAECA & & \\
\hline WS-3 & $8 / 27 / 01$ & BROOK & - & 100 & 9 & CAECA & & \\
\hline WS-3 & $8 / 27 / 01$ & BROOK & $\mathrm{F}$ & 114 & 17 & CAECA & & \\
\hline WS-3 & $8 / 27 / 01$ & BROOK & $F$ & 142 & 28 & CAECA & & \\
\hline WS-3 & $8 / 27 / 01$ & BROOK & M & 115 & 14 & CAECA & & \\
\hline WS-3 & $8 / 27 / 01$ & BROOK & - & 97 & 8 & CAECA & MT-WS3-PYC2 & 442.6 \\
\hline WS-3 & $8 / 27 / 01$ & BROOK & M & 110 & 14 & CAECA & & \\
\hline WS-3 & $8 / 27 / 01$ & BROOK & M & 112 & 13 & CAECA & & \\
\hline WS-3 & $8 / 27 / 01$ & BROOK & - & 105 & 9 & CAECA & & \\
\hline WS-3 & $8 / 27 / 01$ & BROOK & $M$ & 120 & 19 & CAECA & & \\
\hline WS-3 & $8 / 27 / 01$ & BROOK & M & 132 & 26 & CAECA & & \\
\hline WS-3 & $8 / 27 / 01$ & BROOK & $\mathrm{F}$ & 100 & 9 & CAECA & MT-WS3-PYC3 & 345.3 \\
\hline WS-3 & $8 / 27 / 01$ & BROOK & - & 105 & 9 & CAECA & & \\
\hline WS-3 & $8 / 27 / 01$ & BROOK & M & 102 & 10 & CAECA & & \\
\hline WS-3 & $8 / 27 / 01$ & BROOK & - & 108 & 12 & CAECA & & \\
\hline WS-3 & $8 / 27 / 01$ & BROOK & - & 111 & 13 & CAECA & & \\
\hline WS-3 & $8 / 27 / 01$ & BROOK & $M$ & 125 & 20 & CAECA & & \\
\hline WS-3 & $8 / 27 / 01$ & BROOK & M & 112 & 12 & CAECA & MT-WS3- PYC4 & 640.0 \\
\hline WS-3 & $8 / 27 / 01$ & BROOK & - & 109 & 10 & CAECA & & \\
\hline WS-3 & $8 / 27 / 01$ & BROOK & M & 110 & 13 & CAECA & & \\
\hline WS-3 & $8 / 27 / 01$ & BROOK & - & 105 & 9 & CAECA & & \\
\hline WS-3 & $8 / 27 / 01$ & BROOK & - & 98 & 9 & CAECA & & \\
\hline WS-3 & $8 / 27 / 01$ & BROOK & $M$ & 127 & 23 & FILLET & & \\
\hline WS-3 & $8 / 27 / 01$ & BROOK & - & 93 & 10 & FILLET & & \\
\hline WS-3 & $8 / 27 / 01$ & BROOK & $\mathrm{F}$ & 111 & 12 & FILLET & MT-WS3-FIL1 & 11.4 \\
\hline WS-3 & $8 / 27 / 01$ & BROOK & $\mathrm{F}$ & 114 & 15 & FILLET & & \\
\hline WS-3 & $8 / 27 / 01$ & BROOK & - & 100 & 9 & FILLET & & \\
\hline WS-3 & $8 / 27 / 01$ & BROOK & $\mathrm{F}$ & 114 & 17 & FILLET & & \\
\hline WS-3 & $8 / 27 / 01$ & BROOK & $\mathrm{F}$ & 142 & 28 & FILLET & & \\
\hline WS-3 & $8 / 27 / 01$ & BROOK & $M$ & 115 & 14 & FILLET & & \\
\hline WS-3 & $8 / 27 / 01$ & BROOK & - & 97 & 8 & FILLET & MT-WS3-FIL2 & - \\
\hline WS-3 & $8 / 27 / 01$ & BROOK & M & 110 & 14 & FILLET & & \\
\hline WS-3 & $8 / 27 / 01$ & BROOK & M & 112 & 13 & FILLET & & \\
\hline WS-3 & $8 / 27 / 01$ & BROOK & - & 105 & 9 & FILLET & & \\
\hline WS-3 & $8 / 27 / 01$ & BROOK & $M$ & 120 & 19 & FILLET & & \\
\hline WS-3 & $8 / 27 / 01$ & BROOK & M & 132 & 26 & FILLET & & \\
\hline WS-3 & $8 / 27 / 01$ & BROOK & $\mathrm{F}$ & 100 & 9 & FILLET & MT-WS3-FIL3 & 20.0 \\
\hline WS-3 & $8 / 27 / 01$ & BROOK & - & 105 & 9 & FILLET & & \\
\hline WS-3 & $8 / 27 / 01$ & BROOK & M & 102 & 10 & FILLET & & \\
\hline WS-3 & $8 / 27 / 01$ & BROOK & - & 108 & 12 & FILLET & & \\
\hline WS-3 & $8 / 27 / 01$ & BROOK & - & 111 & 13 & FILLET & & \\
\hline WS-3 & $8 / 27 / 01$ & BROOK & $M$ & 125 & 20 & FILLET & & \\
\hline WS-3 & $8 / 27 / 01$ & BROOK & $M$ & 112 & 12 & FILLET & MT-WS3- FIL4 & 37.2 \\
\hline WS-3 & $8 / 27 / 01$ & BROOK & - & 109 & 10 & FILLET & & \\
\hline WS-3 & $8 / 27 / 01$ & BROOK & $M$ & 110 & 13 & FILLET & & \\
\hline WS-3 & $8 / 27 / 01$ & BROOK & - & 105 & 9 & FILLET & & \\
\hline WS-3 & $8 / 27 / 01$ & BROOK & - & 98 & 9 & FILLET & & \\
\hline
\end{tabular}


Trout Population Data

\begin{tabular}{|c|c|c|c|c|c|}
\hline Site ID & Date & Species & Length (mm) & Weight (g) & $\begin{array}{c}\text { Biomass } \\
\text { (kg/hectare) }\end{array}$ \\
\hline PP-1 & $8 / 29 / 2001$ & BROOK & 124 & 19 & \\
\hline PP-1 & $8 / 29 / 2001$ & BROOK & 208 & 121 & \\
\hline PP-1 & $8 / 29 / 2001$ & BROOK & 205 & 95 & \\
\hline PP-1 & $8 / 29 / 2001$ & BROOK & 172 & 58 & \\
\hline PP-1 & 8/29/2001 & BROOK & 184 & 67 & \\
\hline PP-1 & $8 / 29 / 2001$ & BROOK & 154 & 38 & \\
\hline PP-1 & $8 / 29 / 2001$ & BROOK & 166 & 42 & \\
\hline PP-1 & $8 / 29 / 2001$ & BROOK & 154 & 32 & \\
\hline PP-1 & $8 / 29 / 2001$ & BROOK & 121 & 15 & \\
\hline PP-1 & 8/29/2001 & BROOK & 122 & 18 & \\
\hline PP-1 & $8 / 29 / 2001$ & BROOK & 132 & 25 & \\
\hline PP-1 & $8 / 29 / 2001$ & BROOK & 103 & 11 & \\
\hline PP-1 & $8 / 29 / 2001$ & BROOK & 156 & 36 & \\
\hline PP-1 & $8 / 29 / 2001$ & BROOK & 173 & 56 & \\
\hline PP-1 & $8 / 29 / 2001$ & BROOK & 128 & 21 & \\
\hline PP-1 & $8 / 29 / 2001$ & BROOK & 114 & 14 & \\
\hline PP-1 & $8 / 29 / 2001$ & BROOK & 102 & 12 & \\
\hline PP-1 & $8 / 29 / 2001$ & BROOK & 184 & 80 & \\
\hline PP-1 & $8 / 29 / 2001$ & BROOK & 154 & 39 & \\
\hline PP-1 & $8 / 29 / 2001$ & BROOK & 174 & 54 & \\
\hline PP-1 & $8 / 29 / 2001$ & BROOK & 196 & 84 & \\
\hline PP-1 & $8 / 29 / 2001$ & BROOK & 196 & 78 & \\
\hline PP-1 & $8 / 29 / 2001$ & BROOK & 119 & 16 & \\
\hline PP-1 & $8 / 29 / 2001$ & BROOK & 190 & 73 & \\
\hline PP-1 & $8 / 29 / 2001$ & BROOK & 120 & 17 & \\
\hline PP-1 & $8 / 29 / 2001$ & BROOK & 124 & 21 & \\
\hline PP-1 & $8 / 29 / 2001$ & BROOK & 96 & 10 & \\
\hline PP-1 & $8 / 29 / 2001$ & BROOK & 150 & 35 & \\
\hline PP-1 & $8 / 29 / 2001$ & BROOK & 160 & 37 & \\
\hline PP-1 & $8 / 29 / 2001$ & BROOK & 105 & 10 & \\
\hline PP-1 & $8 / 29 / 2001$ & BROOK & 198 & 83 & \\
\hline PP-1 & $8 / 29 / 2001$ & BROOK & 205 & 97 & \\
\hline PP-1 & $8 / 29 / 2001$ & BROOK & 110 & 13 & \\
\hline PP-1 & $8 / 29 / 2001$ & BROOK & 122 & 18 & \\
\hline PP-1 & $8 / 29 / 2001$ & BROOK & 124 & 17 & \\
\hline PP-1 & 8/29/2001 & BROOK & 122 & 18 & \\
\hline PP-1 & $8 / 29 / 2001$ & BROOK & 104 & 11 & \\
\hline PP-1 & $8 / 29 / 2001$ & BROOK & 102 & 12 & \\
\hline PP-1 & $8 / 29 / 2001$ & BROOK & 124 & 19 & \\
\hline PP-1 & $8 / 29 / 2001$ & BROOK & 114 & 14 & \\
\hline PP-1 & 8/29/2001 & BROOK & 112 & 15 & \\
\hline PP-1 & $8 / 29 / 2001$ & BROOK & 180 & 64 & \\
\hline PP-1 & $8 / 29 / 2001$ & BROOK & 124 & 18 & \\
\hline PP-1 & $8 / 29 / 2001$ & BROOK & 222 & 129 & \\
\hline PP-1 & $8 / 29 / 2001$ & BROOK & 100 & 10 & \\
\hline PP-1 & $8 / 29 / 2001$ & BROOK & 110 & 14 & \\
\hline PP-1 & $8 / 29 / 2001$ & BROOK & 104 & 12 & \\
\hline PP-1 & $8 / 29 / 2001$ & BROOK & 164 & 42 & \\
\hline PP-1 & $8 / 29 / 2001$ & BROOK & 119 & 14 & \\
\hline PP-1 & $8 / 29 / 2001$ & BROOK & 110 & 15 & \\
\hline PP-1 & $8 / 29 / 2001$ & BROOK & 121 & 19 & \\
\hline PP-1 & $8 / 29 / 2001$ & BROOK & 122 & 17 & \\
\hline PP-1 & 8/29/2001 & BROOK & 100 & 10 & \\
\hline PP-1 & $8 / 29 / 2001$ & BROOK & 107 & 10 & \\
\hline PP-1 & $8 / 29 / 2001$ & BROOK & 108 & 11 & \\
\hline PP-1 & $8 / 29 / 2001$ & BROOK & 103 & 10 & \\
\hline
\end{tabular}


Trout Population Data (Continued)

\begin{tabular}{|c|c|c|c|c|c|}
\hline Site ID & Date & Species & Length (mm) & Weight (g) & $\begin{array}{c}\text { Biomass } \\
\text { (kg/hectare) }\end{array}$ \\
\hline$\overline{\mathrm{PP}-1}$ & 8/29/2001 & BROOK & 122 & 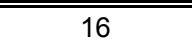 & \\
\hline PP-1 & $8 / 29 / 2001$ & BROOK & 164 & 45 & \\
\hline PP-1 & $8 / 29 / 2001$ & BROOK & 114 & 15 & \\
\hline PP-1 & $8 / 29 / 2001$ & BROOK & 108 & 14 & \\
\hline PP-1 & $8 / 29 / 2001$ & BROOK & 108 & 12 & \\
\hline PP-1 & $8 / 29 / 2001$ & BROOK & 142 & 32 & \\
\hline PP-1 & $8 / 29 / 2001$ & BROOK & 201 & 105 & \\
\hline PP-1 & $8 / 29 / 2001$ & BROOK & 115 & 16 & \\
\hline PP-1 & $8 / 29 / 2001$ & BROOK & 139 & 27 & \\
\hline PP-1 & $8 / 29 / 2001$ & BROOK & 122 & 22 & \\
\hline PP-1 & $8 / 29 / 2001$ & BROOK & 125 & 21 & \\
\hline PP-1 & $8 / 29 / 2001$ & BROOK & 111 & 14 & \\
\hline PP-1 & $8 / 29 / 2001$ & BROOK & 104 & 10 & \\
\hline PP-1 & $8 / 29 / 2001$ & BROOK & 110 & 12 & \\
\hline PP-1 & $8 / 29 / 2001$ & BROOK & 176 & 63 & \\
\hline PP-1 & $8 / 29 / 2001$ & BROOK & 162 & 37 & \\
\hline PP-1 & $8 / 29 / 2001$ & BROOK & 104 & 12 & \\
\hline PP-1 & $8 / 29 / 2001$ & BROOK & 109 & 13 & \\
\hline PP-1 & $8 / 29 / 2001$ & BROOK & 122 & 19 & \\
\hline PP-1 & $8 / 29 / 2001$ & BROOK & 128 & 23 & \\
\hline PP-1 & $8 / 29 / 2001$ & BROOK & 115 & 13 & \\
\hline PP-1 & $8 / 29 / 2001$ & BROOK & 120 & 16 & \\
\hline PP-1 & $8 / 29 / 2001$ & BROOK & 216 & 96 & \\
\hline PP-1 & $8 / 29 / 2001$ & BROOK & 96 & 9 & \\
\hline PP-1 & $8 / 29 / 2001$ & BROOK & 174 & 54 & \\
\hline PP-1 & $8 / 29 / 2001$ & BROOK & 196 & 84 & \\
\hline PP-1 & $8 / 29 / 2001$ & BROOK & 196 & 78 & \\
\hline PP-1 & $8 / 29 / 2001$ & BROOK & 119 & 16 & \\
\hline PP-1 & $8 / 29 / 2001$ & BROOK & 190 & 73 & \\
\hline PP-1 & $8 / 29 / 2001$ & BROOK & 120 & 17 & \\
\hline PP-1 & $8 / 29 / 2001$ & BROOK & 124 & 21 & \\
\hline PP-1 & $8 / 29 / 2001$ & BROOK & 96 & 10 & \\
\hline PP-1 & $8 / 29 / 2001$ & BROOK & 150 & 35 & \\
\hline PP-1 & $8 / 29 / 2001$ & BROOK & 160 & 37 & \\
\hline PP-1 & $8 / 29 / 2001$ & BROOK & 105 & 10 & \\
\hline PP-1 & $8 / 29 / 2001$ & BROOK & 198 & 83 & \\
\hline PP-1 & $8 / 29 / 2001$ & BROOK & 205 & 97 & \\
\hline PP-1 & $8 / 29 / 2001$ & BROOK & 110 & 13 & \\
\hline PP-1 & $8 / 29 / 2001$ & BROOK & 122 & 18 & \\
\hline PP-1 & $8 / 29 / 2001$ & BROOK & 124 & 17 & \\
\hline PP-1 & $8 / 29 / 2001$ & BROOK & 122 & 18 & \\
\hline PP-1 & $8 / 29 / 2001$ & BROOK & 104 & 11 & \\
\hline PP-1 & $8 / 29 / 2001$ & BROOK & 102 & 12 & \\
\hline PP-1 & $8 / 29 / 2001$ & BROOK & 124 & 19 & \\
\hline PP-1 & $8 / 29 / 2001$ & MEAN (all species) & 137 & 33 & 81 \\
\hline PP-3 & 8/28/2001 & BROOK & 244 & 140 & \\
\hline PP-3 & $8 / 28 / 2001$ & BROOK & 188 & 79 & \\
\hline PP-3 & $8 / 28 / 2001$ & BROOK & 160 & 65 & \\
\hline PP-3 & $8 / 28 / 2001$ & BROOK & 164 & 74 & \\
\hline PP-3 & $8 / 28 / 2001$ & BROOK & 168 & 47 & \\
\hline PP-3 & $8 / 28 / 2001$ & BROOK & 142 & 33 & \\
\hline PP-3 & $8 / 28 / 2001$ & BROOK & 144 & 34 & \\
\hline PP-3 & $8 / 28 / 2001$ & BROOK & 148 & 35 & \\
\hline PP-3 & $8 / 28 / 2001$ & BROOK & 150 & 36 & \\
\hline PP-3 & $8 / 28 / 2001$ & BROOK & 138 & 35 & \\
\hline PP-3 & $8 / 28 / 2001$ & BROOK & 150 & 36 & \\
\hline
\end{tabular}


Trout Population Data (Continued)

\begin{tabular}{|c|c|c|c|c|c|}
\hline Site ID & Date & Species & Length (mm) & Weight (g) & $\begin{array}{c}\text { Biomass } \\
\text { (kg/hectare) }\end{array}$ \\
\hline PP-3 & $8 / 28 / 2001$ & BROOK & 146 & 25 & \\
\hline PP-3 & $8 / 28 / 2001$ & BROOK & 96 & 11 & \\
\hline PP-3 & $8 / 28 / 2001$ & BROOK & 128 & 26 & \\
\hline PP-3 & 8/28/2001 & BROOK & 94 & 11 & \\
\hline PP-3 & 8/28/2001 & BROOK & 98 & 12 & \\
\hline PP-3 & $8 / 28 / 2001$ & BROOK & 98 & 13 & \\
\hline PP-3 & $8 / 28 / 2001$ & BROOK & 92 & 13 & \\
\hline PP-3 & 8/28/2001 & BROOK & 98 & 15 & \\
\hline PP-3 & $8 / 28 / 2001$ & BROOK & 92 & 10 & \\
\hline PP-3 & 8/28/2001 & BROOK & 90 & 8 & \\
\hline PP-3 & 8/28/2001 & BROWN & 439 & 839 & \\
\hline PP-3 & $8 / 28 / 2001$ & BROWN & 294 & 248 & \\
\hline PP-3 & 8/28/2001 & BROWN & 269 & 221 & \\
\hline PP-3 & 8/28/2001 & BROWN & 260 & 158 & \\
\hline PP-3 & 8/28/2001 & BROWN & 262 & 187 & \\
\hline PP-3 & 8/28/2001 & BROWN & 210 & 109 & \\
\hline PP-3 & 8/28/2001 & BROWN & 240 & 153 & \\
\hline PP-3 & $8 / 28 / 2001$ & BROWN & 228 & 118 & \\
\hline PP-3 & 8/28/2001 & BROWN & 230 & 128 & \\
\hline PP-3 & 8/28/2001 & BROWN & 264 & 189 & \\
\hline PP-3 & 8/28/2001 & BROWN & 242 & 148 & \\
\hline PP-3 & $8 / 28 / 2001$ & BROWN & 208 & 90 & \\
\hline PP-3 & $8 / 28 / 2001$ & BROWN & 214 & 114 & \\
\hline PP-3 & 8/28/2001 & BROWN & 198 & 77 & \\
\hline PP-3 & 8/28/2001 & BROWN & 150 & 55 & \\
\hline PP-3 & 8/28/2001 & BROWN & 220 & 101 & \\
\hline PP-3 & 8/28/2001 & BROWN & 154 & 46 & \\
\hline PP-3 & $8 / 28 / 2001$ & BROWN & 200 & 73 & \\
\hline PP-3 & 8/28/2001 & BROWN & 152 & 37 & \\
\hline PP-3 & 8/28/2001 & BROWN & 184 & 63 & \\
\hline PP-3 & $8 / 28 / 2001$ & BROWN & 144 & 33 & \\
\hline PP-3 & 8/28/2001 & BROWN & 154 & 50 & \\
\hline PP-3 & $8 / 28 / 2001$ & BROWN & 142 & 31 & \\
\hline PP-3 & 8/28/2001 & BROWN & 136 & 24 & \\
\hline PP-3 & 8/28/2001 & BROWN & 152 & 34 & \\
\hline PP-3 & 8/28/2001 & BROWN & 126 & 22 & \\
\hline PP-3 & 8/28/2001 & BROWN & 128 & 21 & \\
\hline PP-3 & $8 / 28 / 2001$ & RAINBOW & 264 & 212 & \\
\hline PP-3 & 8/28/2001 & RAINBOW & 298 & 240 & \\
\hline PP-3 & $8 / 28 / 2001$ & RAINBOW & 258 & 206 & \\
\hline PP-3 & 8/28/2001 & RAINBOW & 308 & 254 & \\
\hline PP-3 & 8/28/2001 & RAINBOW & 200 & 75 & \\
\hline PP-3 & 8/28/2001 & RAINBOW & 220 & 111 & \\
\hline PP-3 & 8/28/2001 & RAINBOW & 240 & 142 & \\
\hline PP-3 & $8 / 28 / 2001$ & RAINBOW & 262 & 179 & \\
\hline PP-3 & 8/28/2001 & RAINBOW & 298 & 219 & \\
\hline PP-3 & 8/28/2001 & RAINBOW & 160 & 62 & \\
\hline PP-3 & 8/28/2001 & RAINBOW & 122 & 28 & \\
\hline PP-3 & 8/28/2001 & RAINBOW & 130 & 24 & \\
\hline PP-3 & 8/28/2001 & RAINBOW & 115 & 19 & \\
\hline PP-3 & $8 / 28 / 2001$ & RAINBOW & 114 & 19 & \\
\hline PP-3 & $8 / 28 / 2001$ & RAINBOW & 120 & 21 & \\
\hline PP-3 & 8/28/2001 & BROOK & 166 & 44 & \\
\hline PP-3 & $8 / 28 / 2001$ & BROOK & 160 & 40 & \\
\hline PP-3 & $8 / 28 / 2001$ & BROOK & 90 & 7 & \\
\hline PP-3 & $8 / 28 / 2001$ & BROOK & 156 & 37 & \\
\hline
\end{tabular}


Trout Population Data (Continued)

\begin{tabular}{|c|c|c|c|c|c|}
\hline Site ID & Date & Species & Length (mm) & Weight (g) & $\begin{array}{c}\text { Biomass } \\
\text { (kg/hectare) }\end{array}$ \\
\hline PP-3 & $8 / 28 / 2001$ & BROOK & 90 & 8 & \\
\hline PP-3 & $8 / 28 / 2001$ & BROWN & 236 & 130 & \\
\hline PP-3 & $8 / 28 / 2001$ & BROWN & 226 & 130 & \\
\hline PP-3 & $8 / 28 / 2001$ & BROWN & 150 & 31 & \\
\hline PP-3 & $8 / 28 / 2001$ & BROWN & 144 & 28 & \\
\hline PP-3 & $8 / 28 / 2001$ & BROWN & 160 & 40 & \\
\hline PP-3 & $8 / 28 / 2001$ & BROWN & 132 & 24 & \\
\hline PP-3 & $8 / 28 / 2001$ & BROWN & 126 & 18 & \\
\hline PP-3 & $8 / 28 / 2001$ & RAINBOW & 130 & 20 & \\
\hline PP-3 & $8 / 28 / 2001$ & RAINBOW & 166 & 42 & \\
\hline PP-3 & $8 / 28 / 2001$ & RAINBOW & 134 & 24 & \\
\hline PP-3 & $8 / 28 / 2001$ & RAINBOW & 132 & 21 & \\
\hline PP-3 & $8 / 28 / 2001$ & RAINBOW & 134 & 24 & \\
\hline PP-3 & $8 / 28 / 2001$ & MEAN (all species) & 176 & 83 & 167 \\
\hline$\overline{\mathrm{PP}-5}$ & 8/28/2001 & BROWN & 161 & 39 & \\
\hline PP-5 & $8 / 28 / 2001$ & BROWN & 151 & 36 & \\
\hline PP-5 & $8 / 28 / 2001$ & BROWN & 162 & 38 & \\
\hline PP-5 & $8 / 28 / 2001$ & BROWN & 240 & 122 & \\
\hline PP-5 & $8 / 28 / 2001$ & BROWN & 161 & 38 & \\
\hline PP-5 & $8 / 28 / 2001$ & BROWN & 170 & 53 & \\
\hline PP-5 & $8 / 28 / 2001$ & BROWN & 159 & 30 & \\
\hline PP-5 & $8 / 28 / 2001$ & BROWN & 290 & 178 & \\
\hline PP-5 & $8 / 28 / 2001$ & RAINBOW & 181 & 59 & \\
\hline PP-5 & $8 / 28 / 2001$ & RAINBOW & 160 & 42 & \\
\hline PP-5 & $8 / 28 / 2001$ & RAINBOW & 145 & 29 & \\
\hline PP-5 & $8 / 28 / 2001$ & RAINBOW & 163 & 45 & \\
\hline PP-5 & $8 / 28 / 2001$ & RAINBOW & 161 & 38 & \\
\hline PP-5 & $8 / 28 / 2001$ & RAINBOW & 190 & 75 & \\
\hline PP-5 & $8 / 28 / 2001$ & RAINBOW & 154 & 34 & \\
\hline PP-5 & $8 / 28 / 2001$ & RAINBOW & 152 & 31 & \\
\hline PP-5 & $8 / 28 / 2001$ & RAINBOW & 142 & 26 & \\
\hline PP-5 & $8 / 28 / 2001$ & RAINBOW & 146 & 30 & \\
\hline PP-5 & $8 / 28 / 2001$ & RAINBOW & 244 & 108 & \\
\hline PP-5 & $8 / 28 / 2001$ & RAINBOW & 145 & 32 & \\
\hline PP-5 & $8 / 28 / 2001$ & RAINBOW & 159 & 38 & \\
\hline PP-5 & $8 / 28 / 2001$ & RAINBOW & 153 & 37 & \\
\hline PP-5 & $8 / 28 / 2001$ & RAINBOW & 151 & 34 & \\
\hline PP-5 & $8 / 28 / 2001$ & RAINBOW & 220 & 105 & \\
\hline PP-5 & $8 / 28 / 2001$ & RAINBOW & 131 & 23 & \\
\hline PP-5 & $8 / 28 / 2001$ & RAINBOW & 136 & 24 & \\
\hline PP-5 & $8 / 28 / 2001$ & RAINBOW & 172 & 48 & \\
\hline PP-5 & $8 / 28 / 2001$ & RAINBOW & 138 & 28 & \\
\hline PP-5 & $8 / 28 / 2001$ & RAINBOW & 137 & 27 & \\
\hline PP-5 & $8 / 28 / 2001$ & RAINBOW & 166 & 48 & \\
\hline PP-5 & $8 / 28 / 2001$ & RAINBOW & 183 & 65 & \\
\hline PP-5 & $8 / 28 / 2001$ & RAINBOW & 152 & 35 & \\
\hline PP-5 & $8 / 28 / 2001$ & RAINBOW & 143 & 31 & \\
\hline PP-5 & $8 / 28 / 2001$ & BROWN & 170 & 43 & \\
\hline PP-5 & $8 / 28 / 2001$ & RAINBOW & 172 & 50 & \\
\hline PP-5 & $8 / 28 / 2001$ & MEAN (all species) & 167 & 49 & 24 \\
\hline$\overline{\mathrm{L}-1 \mathrm{a}}$ & $8 / 26 / 2001$ & CUTTHROAT & 154 & 29 & \\
\hline L-1a & $8 / 26 / 2001$ & CUTTHROAT & 124 & 15 & \\
\hline L-1a & $8 / 26 / 2001$ & CUTTHROAT & 127 & 17 & \\
\hline L-1a & $8 / 26 / 2001$ & CUTTHROAT & 105 & 10 & \\
\hline$L-1 a$ & $8 / 26 / 2001$ & CUTTHROAT & 120 & 16 & \\
\hline L-1a & $8 / 26 / 2001$ & CUTTHROAT & 130 & 20 & \\
\hline
\end{tabular}


Trout Population Data (Continued)

\begin{tabular}{|c|c|c|c|c|c|}
\hline Site ID & Date & Species & Length (mm) & Weight (g) & $\begin{array}{c}\text { Biomass } \\
\text { (kg/hectare) }\end{array}$ \\
\hline L-1a & $8 / 26 / 2001$ & CUTTHROAT & 85 & 5 & \\
\hline L-1a & $8 / 26 / 2001$ & CUTTHROAT & 71 & 3 & \\
\hline L-1a & $8 / 26 / 2001$ & CUTTHROAT & 102 & 9 & \\
\hline L-1a & $8 / 26 / 2001$ & CUTTHROAT & 75 & 4 & \\
\hline L-1a & $8 / 26 / 2001$ & CUTTHROAT & 95 & 7 & \\
\hline L-1a & 8/26/2001 & CUTTHROAT & 82 & 4 & \\
\hline L-1a & $8 / 26 / 2001$ & CUTTHROAT & 104 & 8 & \\
\hline L-1a & $8 / 26 / 2001$ & CUTTHROAT & 101 & 6 & \\
\hline L-1a & $8 / 26 / 2001$ & CUTTHROAT & 69 & 3 & \\
\hline L-1a & $8 / 26 / 2001$ & CUTTHROAT & 67 & 2 & \\
\hline L-1a & 8/26/2001 & CUTTHROAT & 70 & 3 & \\
\hline L-1a & $8 / 26 / 2001$ & CUTTHROAT & 70 & 3 & \\
\hline L-1a & $8 / 26 / 2001$ & CUTTHROAT & 80 & 4 & \\
\hline L-1a & $8 / 26 / 2001$ & CUTTHROAT & 71 & 3 & \\
\hline L-1a & $8 / 26 / 2001$ & CUTTHROAT & 60 & 2 & \\
\hline L-1a & $8 / 26 / 2001$ & CUTTHROAT & 65 & 2 & \\
\hline L-1a & 8/26/2001 & CUTTHROAT & 70 & 3 & \\
\hline L-1a & $8 / 26 / 2001$ & CUTTHROAT & 75 & 4 & \\
\hline L-1a & 8/26/2001 & CUTTHROAT & 70 & 3 & \\
\hline L-1a & $8 / 26 / 2001$ & CUTTHROAT & 110 & 11 & \\
\hline L-1a & 8/26/2001 & CUTTHROAT & 140 & 20 & \\
\hline L-1a & $8 / 26 / 2001$ & CUTTHROAT & 100 & 9 & \\
\hline L-1a & $8 / 26 / 2001$ & CUTTHROAT & 98 & 8 & \\
\hline L-1a & 8/26/2001 & CUTTHROAT & 98 & 9 & \\
\hline L-1a & $8 / 26 / 2001$ & CUTTHROAT & 65 & 3 & \\
\hline L-1a & $8 / 26 / 2001$ & CUTTHROAT & 86 & 4 & \\
\hline L-1a & $8 / 26 / 2001$ & CUTTHROAT & 71 & 3 & \\
\hline L-1a & $8 / 26 / 2001$ & CUTTHROAT & 84 & 4 & \\
\hline L-1a & $8 / 26 / 2001$ & CUTTHROAT & 77 & 4 & \\
\hline L-1a & $8 / 26 / 2001$ & CUTTHROAT & 72 & 3 & \\
\hline L-1a & $8 / 26 / 2001$ & CUTTHROAT & 68 & 3 & \\
\hline L-1a & $8 / 26 / 2001$ & CUTTHROAT & 72 & 4 & \\
\hline L-1a & $8 / 26 / 2001$ & CUTTHROAT & 128 & 19 & \\
\hline L-1a & $8 / 26 / 2001$ & CUTTHROAT & 100 & 9 & \\
\hline L-1a & $8 / 26 / 2001$ & MEAN (all species) & 90 & 7 & 17 \\
\hline L-5 & $8 / 30 / 2001$ & BROOK & 117 & 15 & \\
\hline L-5 & $8 / 30 / 2001$ & BROOK & 120 & 16 & \\
\hline L-5 & $8 / 30 / 2001$ & BROOK & 97 & 9 & \\
\hline L-5 & $8 / 30 / 2001$ & BROOK & 137 & 24 & \\
\hline L-5 & $8 / 30 / 2001$ & BROOK & 157 & 37 & \\
\hline L-5 & $8 / 30 / 2001$ & BROOK & 127 & 24 & \\
\hline L-5 & $8 / 30 / 2001$ & BROOK & 128 & 20 & \\
\hline L-5 & $8 / 30 / 2001$ & BROOK & 163 & 41 & \\
\hline$L-5$ & $8 / 30 / 2001$ & BROOK & 111 & 12 & \\
\hline L-5 & $8 / 30 / 2001$ & BROOK & 134 & 22 & \\
\hline L-5 & $8 / 30 / 2001$ & BROOK & 120 & 18 & \\
\hline L-5 & $8 / 30 / 2001$ & BROOK & 115 & 14 & \\
\hline L-5 & $8 / 30 / 2001$ & BROOK & 117 & 14 & \\
\hline L-5 & $8 / 30 / 2001$ & BROOK & 98 & 7 & \\
\hline L-5 & $8 / 30 / 2001$ & BROOK & 129 & 25 & \\
\hline L-5 & $8 / 30 / 2001$ & BROOK & 156 & 36 & \\
\hline L-5 & $8 / 30 / 2001$ & BROOK & 175 & 62 & \\
\hline L-5 & $8 / 30 / 2001$ & BROOK & 93 & 8 & \\
\hline$L-5$ & 8/30/2001 & BROOK & 142 & 20 & \\
\hline L-5 & $8 / 30 / 2001$ & BROOK & 90 & 6 & \\
\hline L-5 & $8 / 30 / 2001$ & BROOK & 170 & 51 & \\
\hline
\end{tabular}


Trout Population Data (Continued)

\begin{tabular}{|c|c|c|c|c|c|}
\hline Site ID & Date & Species & Length (mm) & Weight (g) & $\begin{array}{c}\text { Biomass } \\
\text { (kg/hectare) }\end{array}$ \\
\hline$\overline{L-5}$ & $8 / 30 / 2001$ & BROOK & 112 & 13 & \\
\hline L-5 & 8/30/2001 & BROOK & 113 & 12 & \\
\hline L-5 & 8/30/2001 & BROOK & 99 & 7 & \\
\hline L-5 & 8/30/2001 & BROOK & 95 & 5 & \\
\hline L-5 & 8/30/2001 & BROOK & 106 & 10 & \\
\hline L-5 & 8/30/2001 & BROOK & 115 & 12 & \\
\hline L-5 & 8/30/2001 & BROOK & 127 & 21 & \\
\hline$L-5$ & 8/30/2001 & BROOK & 102 & 9 & \\
\hline L-5 & 8/30/2001 & BROOK & 139 & 25 & \\
\hline L-5 & $8 / 30 / 2001$ & BROOK & 143 & 27 & \\
\hline$L-5$ & 8/30/2001 & BROOK & 144 & 26 & \\
\hline L-5 & $8 / 30 / 2001$ & BROOK & 143 & 26 & \\
\hline$L-5$ & 8/30/2001 & BROOK & 102 & 8 & \\
\hline L-5 & 8/30/2001 & BROOK & 134 & 20 & \\
\hline L-5 & 8/30/2001 & BROOK & 103 & 8 & \\
\hline L-5 & $8 / 30 / 2001$ & BROOK & 102 & 8 & \\
\hline L-5 & $8 / 30 / 2001$ & BROOK & 111 & 13 & \\
\hline$L-5$ & 8/30/2001 & BROOK & 126 & 15 & \\
\hline L-5 & 8/30/2001 & BROOK & 97 & 8 & \\
\hline L-5 & 8/30/2001 & BROOK & 103 & 9 & \\
\hline L-5 & 8/30/2001 & BROOK & 122 & 16 & \\
\hline L-5 & 8/30/2001 & BROOK & 124 & 20 & \\
\hline L-5 & 8/30/2001 & BROOK & 101 & 8 & \\
\hline L-5 & 8/30/2001 & BROOK & 96 & 8 & \\
\hline L-5 & $8 / 30 / 2001$ & BROOK & 143 & 27 & \\
\hline L-5 & $8 / 30 / 2001$ & BROOK & 124 & 14 & \\
\hline$L-5$ & 8/30/2001 & BROOK & 112 & 12 & \\
\hline L-5 & 8/30/2001 & BROOK & 107 & 11 & \\
\hline L-5 & $8 / 30 / 2001$ & BROOK & 150 & 32 & \\
\hline L-5 & 8/30/2001 & BROOK & 110 & 13 & \\
\hline L-5 & $8 / 30 / 2001$ & BROOK & 90 & 7 & \\
\hline L-5 & 8/30/2001 & BROOK & 106 & 11 & \\
\hline L-5 & $8 / 30 / 2001$ & BROOK & 103 & 10 & \\
\hline$L-5$ & 8/30/2001 & BROOK & 111 & 12 & \\
\hline L-5 & $8 / 30 / 2001$ & BROOK & 114 & 12 & \\
\hline L-5 & $8 / 30 / 2001$ & BROOK & 121 & 15 & \\
\hline$L-5$ & 8/30/2001 & BROOK & 145 & 30 & \\
\hline L-5 & $8 / 30 / 2001$ & BROOK & 91 & 9 & \\
\hline L-5 & 8/30/2001 & BROOK & 136 & 24 & \\
\hline L-5 & 8/30/2001 & BROOK & 128 & 22 & \\
\hline L-5 & $8 / 30 / 2001$ & BROOK & 105 & 9 & \\
\hline L-5 & $8 / 30 / 2001$ & BROOK & 110 & 10 & \\
\hline L-5 & $8 / 30 / 2001$ & BROOK & 120 & 13 & \\
\hline L-5 & 8/30/2001 & BROOK & 120 & 13 & \\
\hline$L-5$ & 8/30/2001 & BROOK & 90 & 6 & \\
\hline L-5 & $8 / 30 / 2001$ & BROOK & 92 & 7 & \\
\hline L-5 & 8/30/2001 & BROOK & 113 & 12 & \\
\hline L-5 & 8/30/2001 & BROOK & 91 & 6 & \\
\hline L-5 & $8 / 30 / 2001$ & BROOK & 100 & 9 & \\
\hline L-5 & $8 / 30 / 2001$ & BROOK & 108 & 10 & \\
\hline L-5 & $8 / 30 / 2001$ & BROOK & 115 & 14 & \\
\hline L-5 & $8 / 30 / 2001$ & BROOK & 122 & 16 & \\
\hline$L-5$ & 8/30/2001 & BROOK & 103 & 7 & \\
\hline L-5 & 8/30/2001 & BROOK & 95 & 7 & \\
\hline L-5 & $8 / 30 / 2001$ & BROOK & 97 & 7 & \\
\hline L-5 & $8 / 30 / 2001$ & BROOK & 82 & 5 & \\
\hline
\end{tabular}


Trout Population Data (Continued)

\begin{tabular}{|c|c|c|c|c|c|}
\hline Site ID & Date & Species & Length (mm) & Weight (g) & $\begin{array}{c}\text { Biomass } \\
\text { (kg/hectare) }\end{array}$ \\
\hline $\mathrm{L}-5$ & $8 / 30 / 2001$ & BROOK & 100 & 8 & \\
\hline L-5 & $8 / 30 / 2001$ & BROOK & 97 & 8 & \\
\hline L-5 & $8 / 30 / 2001$ & BROOK & 136 & 21 & \\
\hline L-5 & $8 / 30 / 2001$ & BROOK & 104 & 10 & \\
\hline L-5 & $8 / 30 / 2001$ & BROOK & 91 & 7 & \\
\hline L-5 & $8 / 30 / 2001$ & BROOK & 94 & 8 & \\
\hline L-5 & $8 / 30 / 2001$ & BROOK & 125 & 16 & \\
\hline L-5 & $8 / 30 / 2001$ & BROOK & 89 & 6 & \\
\hline L-5 & $8 / 30 / 2001$ & BROOK & 99 & 9 & \\
\hline$L-5$ & $8 / 30 / 2001$ & BROOK & 91 & 6 & \\
\hline L-5 & $8 / 30 / 2001$ & BROOK & 118 & 13 & \\
\hline L-5 & $8 / 30 / 2001$ & BROOK & 132 & 19 & \\
\hline L-5 & $8 / 30 / 2001$ & BROOK & 107 & 11 & \\
\hline L-5 & $8 / 30 / 2001$ & BROOK & 99 & 8 & \\
\hline L-5 & $8 / 30 / 2001$ & BROOK & 125 & 18 & \\
\hline L-5 & $8 / 30 / 2001$ & BROOK & 107 & 11 & \\
\hline L-5 & $8 / 30 / 2001$ & BROOK & 112 & 13 & \\
\hline L-5 & $8 / 30 / 2001$ & BROOK & 119 & 16 & \\
\hline L-5 & $8 / 30 / 2001$ & BROOK & 90 & 6 & \\
\hline L-5 & $8 / 30 / 2001$ & BROOK & 104 & 9 & \\
\hline L-5 & $8 / 30 / 2001$ & BROOK & 98 & 9 & \\
\hline L-5 & $8 / 30 / 2001$ & BROOK & 90 & 6 & \\
\hline L-5 & $8 / 30 / 2001$ & BROOK & 109 & 12 & \\
\hline L-5 & $8 / 30 / 2001$ & BROOK & 103 & 10 & \\
\hline L-5 & $8 / 30 / 2001$ & BROOK & 140 & 28 & \\
\hline$L-5$ & $8 / 30 / 2001$ & BROOK & 85 & 6 & \\
\hline L-5 & $8 / 30 / 2001$ & BROOK & 98 & 9 & \\
\hline L-5 & $8 / 30 / 2001$ & BROOK & 99 & 8 & \\
\hline L-5 & $8 / 30 / 2001$ & BROOK & 96 & 9 & \\
\hline L-5 & $8 / 30 / 2001$ & BROOK & 89 & 6 & \\
\hline$L-5$ & $8 / 30 / 2001$ & BROOK & 85 & 5 & \\
\hline L-5 & $8 / 30 / 2001$ & BROOK & 107 & 10 & \\
\hline L-5 & $8 / 30 / 2001$ & BROOK & 117 & 15 & \\
\hline L-5 & $8 / 30 / 2001$ & BROOK & 120 & 16 & \\
\hline $\mathrm{L}-5$ & $8 / 30 / 2001$ & BROOK & 97 & 9 & \\
\hline L-5 & $8 / 30 / 2001$ & MEAN (all species) & 113 & 14 & 57 \\
\hline WS-3 & 8/27/2001 & BROOK & 91 & 6 & \\
\hline WS-3 & $8 / 27 / 2001$ & BROOK & 130 & 27 & \\
\hline WS-3 & $8 / 27 / 2001$ & BROOK & 100 & 9 & \\
\hline WS-3 & $8 / 27 / 2001$ & BROOK & 105 & 10 & \\
\hline WS-3 & $8 / 27 / 2001$ & BROOK & 88 & 6 & \\
\hline WS-3 & $8 / 27 / 2001$ & BROOK & 96 & 8 & \\
\hline WS-3 & $8 / 27 / 2001$ & BROOK & 99 & 10 & \\
\hline WS-3 & $8 / 27 / 2001$ & BROOK & 112 & 13 & \\
\hline WS-3 & $8 / 27 / 2001$ & BROOK & 90 & 6 & \\
\hline WS-3 & $8 / 27 / 2001$ & BROOK & 115 & 17 & \\
\hline WS-3 & $8 / 27 / 2001$ & BROOK & 99 & 9 & \\
\hline WS-3 & $8 / 27 / 2001$ & BROOK & 100 & 12 & \\
\hline WS-3 & $8 / 27 / 2001$ & BROOK & 127 & 24 & \\
\hline WS-3 & $8 / 27 / 2001$ & BROOK & 89 & 7 & \\
\hline WS-3 & $8 / 27 / 2001$ & BROOK & 83 & 5 & \\
\hline WS-3 & $8 / 27 / 2001$ & BROOK & 107 & 10 & \\
\hline WS-3 & $8 / 27 / 2001$ & BROOK & 97 & 10 & \\
\hline WS-3 & $8 / 27 / 2001$ & BROOK & 86 & 6 & \\
\hline WS-3 & $8 / 27 / 2001$ & BROOK & 115 & 16 & \\
\hline WS-3 & $8 / 27 / 2001$ & BROOK & 84 & 6 & \\
\hline
\end{tabular}


Trout Population Data (Continued)

\begin{tabular}{|c|c|c|c|c|c|}
\hline Site ID & Date & Species & Length (mm) & Weight (g) & $\begin{array}{c}\text { Biomass } \\
\text { (kg/hectare) }\end{array}$ \\
\hline WS-3 & $8 / 27 / 2001$ & BROOK & 117 & 19 & \\
\hline WS-3 & $8 / 27 / 2001$ & BROOK & 107 & 12 & \\
\hline WS-3 & $8 / 27 / 2001$ & BROOK & 74 & 4 & \\
\hline WS-3 & $8 / 27 / 2001$ & BROOK & 93 & 8 & \\
\hline WS-3 & $8 / 27 / 2001$ & BROOK & 82 & 5 & \\
\hline WS-3 & $8 / 27 / 2001$ & BROOK & 89 & 9 & \\
\hline WS-3 & $8 / 27 / 2001$ & BROOK & 110 & 12 & \\
\hline WS-3 & $8 / 27 / 2001$ & BROOK & 81 & 5 & \\
\hline WS-3 & $8 / 27 / 2001$ & BROOK & 95 & 9 & \\
\hline WS-3 & $8 / 27 / 2001$ & BROOK & 91 & 6 & \\
\hline WS-3 & $8 / 27 / 2001$ & BROOK & 82 & 6 & \\
\hline WS-3 & $8 / 27 / 2001$ & BROOK & 88 & 7 & \\
\hline WS-3 & $8 / 27 / 2001$ & BROOK & 94 & 8 & \\
\hline WS-3 & $8 / 27 / 2001$ & BROOK & 93 & 8 & \\
\hline WS-3 & $8 / 27 / 2001$ & BROOK & 87 & 6 & \\
\hline WS-3 & $8 / 27 / 2001$ & BROOK & 82 & 5 & \\
\hline WS-3 & $8 / 27 / 2001$ & BROOK & 82 & 6 & \\
\hline WS-3 & $8 / 27 / 2001$ & BROOK & 97 & 7 & \\
\hline WS-3 & $8 / 27 / 2001$ & BROOK & 92 & 9 & \\
\hline WS-3 & $8 / 27 / 2001$ & BROOK & 100 & 10 & \\
\hline WS-3 & $8 / 27 / 2001$ & BROOK & 86 & 5 & \\
\hline WS-3 & $8 / 27 / 2001$ & BROOK & 105 & 10 & \\
\hline WS-3 & $8 / 27 / 2001$ & MEAN (all species) & 96 & 9 & 17 \\
\hline C-5 & 8/30/2001 & BROOK & 129 & 26 & \\
\hline$C-5$ & $8 / 30 / 2001$ & BROOK & 136 & 32 & \\
\hline C-5 & $8 / 30 / 2001$ & BROOK & 129 & 22 & \\
\hline C-5 & $8 / 30 / 2001$ & BROOK & 127 & 21 & \\
\hline C-5 & $8 / 30 / 2001$ & BROOK & 133 & 26 & \\
\hline C-5 & $8 / 30 / 2001$ & BROOK & 217 & 105 & \\
\hline C-5 & $8 / 30 / 2001$ & BROOK & 112 & 13 & \\
\hline C-5 & $8 / 30 / 2001$ & BROOK & 139 & 24 & \\
\hline C-5 & $8 / 30 / 2001$ & BROOK & 176 & 54 & \\
\hline C-5 & $8 / 30 / 2001$ & BROOK & 119 & 18 & \\
\hline$C-5$ & $8 / 30 / 2001$ & BROOK & 132 & 19 & \\
\hline C-5 & $8 / 30 / 2001$ & BROOK & 177 & 57 & \\
\hline C-5 & $8 / 30 / 2001$ & BROOK & 140 & 31 & \\
\hline C-5 & $8 / 30 / 2001$ & BROOK & 114 & 16 & \\
\hline C-5 & $8 / 30 / 2001$ & BROOK & 114 & 15 & \\
\hline$C-5$ & $8 / 30 / 2001$ & BROOK & 134 & 20 & \\
\hline C-5 & $8 / 30 / 2001$ & BROOK & 130 & 22 & \\
\hline C-5 & $8 / 30 / 2001$ & BROOK & 133 & 21 & \\
\hline$C-5$ & $8 / 30 / 2001$ & BROOK & 152 & 38 & \\
\hline C-5 & $8 / 30 / 2001$ & BROOK & 131 & 23 & \\
\hline C-5 & $8 / 30 / 2001$ & BROOK & 125 & 18 & \\
\hline C-5 & $8 / 30 / 2001$ & BROOK & 157 & 38 & \\
\hline$C-5$ & $8 / 30 / 2001$ & BROOK & 165 & 48 & \\
\hline C-5 & $8 / 30 / 2001$ & BROOK & 132 & 24 & \\
\hline C-5 & $8 / 30 / 2001$ & BROOK & 132 & 25 & \\
\hline C-5 & $8 / 30 / 2001$ & BROOK & 133 & 20 & \\
\hline C-5 & $8 / 30 / 2001$ & BROOK & 131 & 22 & \\
\hline$C-5$ & $8 / 30 / 2001$ & BROOK & 120 & 17 & \\
\hline$C-5$ & $8 / 30 / 2001$ & BROWN & 195 & 91 & \\
\hline C-5 & $8 / 30 / 2001$ & BROWN & 132 & 24 & \\
\hline C-5 & $8 / 30 / 2001$ & BROWN & 122 & 19 & \\
\hline C-5 & $8 / 30 / 2001$ & BROWN & 95 & 9 & \\
\hline$C-5$ & 8/30/2001 & BROWN & 85 & 6 & \\
\hline
\end{tabular}


Trout Population Data (Continued)

\begin{tabular}{|c|c|c|c|c|c|}
\hline Site ID & Date & Species & Length (mm) & Weight (g) & $\begin{array}{c}\text { Biomass } \\
\text { (kg/hectare) }\end{array}$ \\
\hline $\mathrm{C}-5$ & $8 / 30 / 2001$ & BROWN & 130 & 23 & \\
\hline C-5 & $8 / 30 / 2001$ & BROWN & 106 & 10 & \\
\hline C-5 & $8 / 30 / 2001$ & BROWN & 118 & 15 & \\
\hline C-5 & 8/30/2001 & BROWN & 198 & 84 & \\
\hline C-5 & 8/30/2001 & BROWN & 87 & 8 & \\
\hline C-5 & 8/30/2001 & BROWN & 179 & 57 & \\
\hline C-5 & $8 / 30 / 2001$ & BROWN & 188 & 60 & \\
\hline C-5 & $8 / 30 / 2001$ & BROWN & 107 & 11 & \\
\hline C-5 & 8/30/2001 & BROWN & 125 & 20 & \\
\hline C-5 & 8/30/2001 & BROWN & 121 & 17 & \\
\hline C-5 & 8/30/2001 & BROWN & 117 & 18 & \\
\hline C-5 & 8/30/2001 & BROWN & 106 & 11 & \\
\hline C-5 & $8 / 30 / 2001$ & BROWN & 127 & 23 & \\
\hline$C-5$ & 8/30/2001 & BROWN & 110 & 13 & \\
\hline$C-5$ & 8/30/2001 & BROWN & 101 & 10 & \\
\hline C-5 & 8/30/2001 & BROWN & 109 & 13 & \\
\hline C-5 & 8/30/2001 & BROWN & 111 & 14 & \\
\hline C-5 & $8 / 30 / 2001$ & BROWN & 90 & 7 & \\
\hline C-5 & 8/30/2001 & BROWN & 107 & 10 & \\
\hline$C-5$ & 8/30/2001 & BROWN & 121 & 18 & \\
\hline C-5 & 8/30/2001 & BROWN & 106 & 12 & \\
\hline C-5 & 8/30/2001 & BROWN & 109 & 12 & \\
\hline C-5 & $8 / 30 / 2001$ & BROWN & 95 & 9 & \\
\hline C-5 & 8/30/2001 & BROWN & 115 & 13 & \\
\hline$C-5$ & 8/30/2001 & BROWN & 114 & 14 & \\
\hline$C-5$ & $8 / 30 / 2001$ & BROWN & 112 & 13 & \\
\hline C-5 & 8/30/2001 & BROWN & 104 & 10 & \\
\hline C-5 & $8 / 30 / 2001$ & BROWN & 100 & 9 & \\
\hline$C-5$ & 8/30/2001 & BROWN & 102 & 10 & \\
\hline$C-5$ & 8/30/2001 & BROWN & 100 & 10 & \\
\hline C-5 & $8 / 30 / 2001$ & BROWN & 117 & 17 & \\
\hline$C-5$ & 8/30/2001 & BROWN & 102 & 10 & \\
\hline C-5 & $8 / 30 / 2001$ & BROWN & 105 & 11 & \\
\hline$C-5$ & $8 / 30 / 2001$ & BROWN & 99 & 9 & \\
\hline$C-5$ & $8 / 30 / 2001$ & BROWN & 87 & 6 & \\
\hline$C-5$ & 8/30/2001 & BROWN & 142 & 25 & \\
\hline$C-5$ & 8/30/2001 & BROWN & 119 & 17 & \\
\hline C-5 & $8 / 30 / 2001$ & BROWN & 100 & 8 & \\
\hline$C-5$ & 8/30/2001 & BROWN & 116 & 15 & \\
\hline$C-5$ & 8/30/2001 & BROWN & 125 & 20 & \\
\hline$C-5$ & 8/30/2001 & BROWN & 92 & 8 & \\
\hline$C-5$ & 8/30/2001 & BROWN & 111 & 11 & \\
\hline$C-5$ & $8 / 30 / 2001$ & RAINBOW & 152 & 32 & \\
\hline$C-5$ & 8/30/2001 & RAINBOW & 107 & 12 & \\
\hline C-5 & $8 / 30 / 2001$ & RAINBOW & 105 & 10 & \\
\hline$C-5$ & 8/30/2001 & BROOK & 133 & 25 & \\
\hline$C-5$ & 8/30/2001 & BROOK & 115 & 12 & \\
\hline$C-5$ & 8/30/2001 & BROWN & 99 & 10 & \\
\hline$C-5$ & 8/30/2001 & BROWN & 100 & 9 & \\
\hline$C-5$ & $8 / 30 / 2001$ & BROWN & 122 & 18 & \\
\hline$C-5$ & 8/30/2001 & BROWN & 98 & 9 & \\
\hline$C-5$ & $8 / 30 / 2001$ & BROWN & 95 & 8 & \\
\hline$C-5$ & $8 / 30 / 2001$ & BROWN & 107 & 11 & \\
\hline$C-5$ & 8/30/2001 & BROWN & 110 & 12 & \\
\hline$C-5$ & 8/30/2001 & BROWN & 100 & 9 & \\
\hline$C-5$ & $8 / 30 / 2001$ & BROWN & 95 & 8 & \\
\hline
\end{tabular}


Trout Population Data (Continued)

\begin{tabular}{|c|c|c|c|c|c|}
\hline Site ID & Date & Species & Length (mm) & Weight (g) & $\begin{array}{c}\text { Biomass } \\
\text { (kg/hectare) }\end{array}$ \\
\hline $\mathrm{C}-5$ & $8 / 30 / 2001$ & RAINBOW & 97 & 9 & \\
\hline C-5 & $8 / 30 / 2001$ & RAINBOW & 86 & 7 & \\
\hline$C-5$ & $8 / 30 / 2001$ & RAINBOW & 94 & 8 & \\
\hline C-5 & $8 / 30 / 2001$ & RAINBOW & 80 & 5 & \\
\hline C-5 & $8 / 30 / 2001$ & RAINBOW & 113 & 13 & \\
\hline$\overline{C-5}$ & $8 / 30 / 2001$ & MEAN (all species) & 121 & 20 & 71 \\
\hline
\end{tabular}




\section{REFERENCES CITED}

Ball, J.W., and Nordstrom, D.K., 1991, User's manual for WATEQ4F, with revised thermodynamic data base and test cases for calculating speciation of major, trace, and redox elements in natural waters: U.S. Geological Survey Open-File Report 91-183, $189 \mathrm{p}$.

Barringer, J.L., and Johnsson, P.A., 1996, Theoretical considerations and a simple method for measuring alkalinity and acidity in low-pH waters by Gran titration:

U.S. Geological Survey Water-Resources Investigations Report 89-4029, 36 p.

Brinton, T.I., Antweiler, R.C., and Taylor, H.E., 1995, Method for the determination of dissolved chloride, nitrate, and sulfate in natural water using ion chromatography: U.S. Geological Survey Open-File Report 95-426A, 16 p.

Briggs, P.H., 1996, Forty elements by inductively coupled-plasma atomic emission spectrometry, in Arbogast, B.F., ed., Analytical methods manual for the Mineral Resources Program, U.S. Geological Survey Open-File Report 96-525, p. 77-94.

Chao, T.T., 1984, Use of partial dissolution techniques in geochemical exploration: Journal of Geochemical Exploration, v. 20, p. 101-135.

Crock, J.G., Lichte, F.E., and Briggs, 1983, Determination of elements in National Bureau of Standards geologic reference materials SRM 278 obsidian and SRM 688 basalt by inductively coupled plasma-atomic emission spectroscopy: Geostandards Newsletter, v.7, p. 335-340.

Church, S.E., Mosier, E.L., and Motooka, J.M., 1987, Mineralogical basis for the interpretation of multielement (ICP-AES), oxalic acid, and aqua-regia partial digestions of stream sediments for reconnaissance exploration geochemistry: Journal of Geochemical Exploration, v. 29, p. 207-233.

Church, S.E., Holmes, C.E., Briggs, P.H., Vaughn, R.B., Cathcart, James and Marot, Margaret, 1993, Geochemical and lead-isotope data from stream and lake sediments, and cores from the upper Arkansas River drainage: Effects of mining at Leadville Colorado on heavy-metal concentrations in the Arkansas River: U.S. Geological Survey Open-File Report 93-534, 61 p.

Church, S.E., Kimball, B.A., Fey, D.L., Ferderer, D.A., Yager, T.J., and Vaughn, R.B., 1997, Source, transport, and partitioning of metals between water, colloids, and bed sediments of the Animas River, Colorado: U.S. Geological Survey Open-File Report 97-151, $135 \mathrm{p}$

Dillard, C. J., and A. L. Tappel. 1984. Fluorescent damage products of lipid peroxidation. Methods of Enzymology v. 105 p. 337-341.

Faires, L.M., 1993, Methods of analysis by the U.S. Geological Survey National Water Quality Laboratory-Determination of metals in water by inductively coupled plasma-mass spectrometry: U.S. Geological Survey Open-File Report 92-634, $28 \mathrm{p}$.

Farag, A.M., D.F. Woodward, J.N. Goldstein, W. Brumbaugh, and J.S. Meyer, 1998, Concentrations of metals associated with mining waste in sediments, biofilm, benthic macroinvertebrates, and fish from the Coeur d'Alene River Basin, Idaho: Archives of Environmental Contamination and Toxicology v. 34, p. 119-127.

Farag, A.M., M.A. Stansbury, C. Hogstrand, E. MacConnell, and H. L. Bergman, 1995, The physiological impairment of free-ranging brown trout exposed to metals in the Clark Fork River, Montana: Canadian Journal of Fisheries and Aquatic Sciences v. 52 p. 2038-2050. 
Fey, D.L., Unruh, D.M., and Church, S.E., 1999a, Chemical data and lead isotopic compositions in stream-sediment samples from the Boulder River watershed, Jefferson County, Montana: U. S. Geological Survey Open-File Report 99-575, $147 \mathrm{p}$

Fey, D.L., Church, S.E., and Finney, C.J., 1999b, Analytical results for 35 mine-waste tailings cores and six bed-sediment samples, and an estimate of the volume of contaminated material at Buckeye Meadow on upper Basin Creek, northern Jefferson County, Montana: U.S. Geological Survey Open-File Report 99-537, 59 p.

Fey, D.L., and Church, S.E., 1998, Analytical results for 42 fluvial tailings cores and 7 stream-sediment samples from High Ore Creek, northern Jefferson County, Montana: U. S. Geological Survey Open-File Report 98-215, 49 p.

Fey, D.L., Church, S.E., and Finney, C.J., 2000, Analytical results for Bullion Mine and Crystal Mine waste samples and bed sediments from a small tributary to Jack Creek and from Uncle Sam Gulch, Boulder River watershed, Montana: U.S. Geological Survey Open-File Report 00-031, 63 p.

Fishman, M.J., ed., 1993, Methods of analysis by the U.S. Geological Survey National Water Quality Laboratory-Determination of inorganic and organic constituents in water and fluvial sediments: U.S. Geological Survey Open-File Report 93-125, $217 \mathrm{p}$.

Fishman, M.J., and Friedman, L.C., eds., 1989, Methods for determination of inorganic substances in water and fluvial sediments: U.S. Geological Survey Techniques of Water-Resources Investigations, book 5, chap. A1, 545 p.

Fletcher, B.L., C.J. Dillard, and A. L. Tappel, 1973, Measurement of fluorescent lipid peroxidation products in biological systems and tissues, Analytical Biochemistry v. 52 p. 1-9.

Friedman, L.C., and Erdmann, D.E., 1982, Quality assurance practices for the chemical and biological analyses of water and fluvial sediments: U.S. Geological Survey Techniques of Water-Resources Investigations, book 5, chap. A6, 181 p.

Garbarino, J.R., 1999, Methods of analysis by the U.S. Geological Survey National Water Quality Laboratory-Determination of dissolved arsenic, boron, lithium, selenium, strontium, thallium, and vanadium using inductively coupled plasmamass spectrometry: U.S. Geological Survey Open-File Report 99-093, 31 p.

Garbarino, J.R., and Struzeski, T.M., 1998, Methods of analysis by the U.S. Geological Survey National Water Quality Laboratory_-Determination of elements in wholewater digests using inductively coupled plasma-optical emission spectrometry and inductively coupled plasma-mass spectrometry: U.S. Geological Survey OpenFile Report 98-165, 101 p.

Garbarino, J.R., and Taylor, H.E., 1996, Inductively coupled plasma-mass spectrometric method for the determination of dissolved trace elements in natural water: U.S. Geological Survey Open-File Report 94-358, 49 p.

Guy, H.P., 1969, Laboratory theory and methods for sediment analysis: U.S. Geological Survey Techniques of Water-Resources Investigations, book 5, chap. C1, 58 p. Hoffman, G.L., Fishman, M.J., and Garbarino, J.R., 1996, Methods of analysis by the U.S. Geological Survey National Water Quality Laboratory-In-bottle acid digestion of whole-water samples: U.S. Geological Survey Open-File Report 96-225, $28 \mathrm{p}$. 
Hogstrand, C., R. W. Wilson, D. Polgar, and C. M. Wood. 1994. Effects of zinc on the kinetics of branchial calcium uptake in freshwater rainbow trout during adaptation to waterborne zinc. Journal of Experimental Biology v. 186 p. 55-73.

Jones, B.E., 1987, Quality control manual of the U.S. Geological Survey's National Water Quality Laboratory: U.S. Geological Survey Open-File Report 87-457, $17 \mathrm{p}$.

Jones, S.R., and Garbarino, J.R., 1999, Methods of analysis by the U.S. Geological Survey National Water Quality Laboratory-Determination of arsenic and selenium in water and sediment by graphite furnace-atomic absorption spectrometry: U.S. Geological Survey Open-File Report 98-639, 39 p.

Klein, T.L., Thamke, J.N., and Farag, A.M., eds., 2001, Water-quality, biology, and streambed sediment data and preliminary geochemical interpretations for streams in the upper Prickly Pear Creek watershed, Montana, 2000: U.S. Geological Survey, Geologic Division, Open-File Report 01-280, 59 p.

Knapton, J.R., and Nimick, D.A., 1991, Quality assurance for water-quality activities of the U.S. Geological Survey in Montana: U.S. Geological Survey Open-File Report 91-216, 41 p.

Lambing, J.H., and Dodge, K.A., 1993, Quality assurance for laboratory analysis of suspended-sediment samples by the U.S. Geological Survey in Montana: U.S. Geological Survey Open-File Report 93-131, 34 p.

McCleskey, D.K., Nordstrom, D.K., and Ball, J.W., 2001, Cation-exchange separation of interfering metals from acid mine waters for accurate determination of total arsenic and arsenic (III) by hydride generation-atomic absorption spectrometry: $43^{\text {rd }}$ Rocky Mountain Conference on Analytical Chemistry, Denver, Colo., July 29-August 2, 2001.

Nimick, D.A., and Cleasby, T.E., 2000, Water-quality data for streams in the Boulder River watershed, Jefferson County, Montana: U.S. Geological Survey Open-File Report 00-99, 70 p.

Pritt, J.W., and Raese, J.W., eds., 1995, Quality assurance/quality control manualNational Water Quality Laboratory: U.S. Geological Survey Open-File Report 95-443, $35 \mathrm{p}$.

Rantz, S.E., and others, 1982, Measurement and computation of streamflow: U.S. Geological Survey Water-Supply Paper 2175, 2 v., 631 p.

Stookey, L.L., 1970, FerroZine-a new spectrophotometric reagent for iron: Analytical Chemistry, v. 42, p. 779-781.

Wilde, F.D., Radtke, D.B., Gibs, Jacob, and Iwatsubo, R.T., 1998, National field manual for the collection of water-quality data: U.S. Geological Survey Techniques of Water-Resources Investigations, book 9, chap. A1-A9.

Wilson, S. A., Briggs, P.H., Mee, J.S., and Siems, D.F., 1994, Determinations of thirtytwo major and trace elements in three NIST soil SRMs using ICP-AES and WDXRF: Geostandards Newsletter, v. 18, p. 85-89

Zippen, C. 1958. The removal method of population estimation: Journal of Wildlife Management, v. 22, p. 82-90. 